\title{
Polyunsaturated fatty acids and the fetal origins hypothesis : a study on cardiovascular risk indicators in children
}

Citation for published version (APA):

Rump, P. (2002). Polyunsaturated fatty acids and the fetal origins hypothesis : a study on cardiovascular risk indicators in children. [Doctoral Thesis, Maastricht University]. Universiteit Maastricht. https://doi.org/10.26481/dis.20021206pr

Document status and date:

Published: 01/01/2002

DOI:

10.26481/dis.20021206pr

Document Version:

Publisher's PDF, also known as Version of record

Please check the document version of this publication:

- A submitted manuscript is the version of the article upon submission and before peer-review. There can be important differences between the submitted version and the official published version of record.

People interested in the research are advised to contact the author for the final version of the publication, or visit the DOI to the publisher's website.

- The final author version and the galley proof are versions of the publication after peer review.

- The final published version features the final layout of the paper including the volume, issue and page numbers.

Link to publication

\footnotetext{
General rights rights.

- You may freely distribute the URL identifying the publication in the public portal. please follow below link for the End User Agreement:

www.umlib.nl/taverne-license

Take down policy

If you believe that this document breaches copyright please contact us at:

repository@maastrichtuniversity.nl

providing details and we will investigate your claim.
}

Copyright and moral rights for the publications made accessible in the public portal are retained by the authors and/or other copyright owners and it is a condition of accessing publications that users recognise and abide by the legal requirements associated with these

- Users may download and print one copy of any publication from the public portal for the purpose of private study or research.

- You may not further distribute the material or use it for any profit-making activity or commercial gain

If the publication is distributed under the terms of Article $25 \mathrm{fa}$ of the Dutch Copyright Act, indicated by the "Taverne" license above, 
Thesis |

Polyunsaturated fatty acids and the fetal origins hypothesis

A study on cardiovascular risk indicators in children 


\section{(ㄷ) 2002 Patrick Rump}

Polyunsaturated fatty acids and the fetal origins hypothesis A study on cardiovascular risk indicators in children Thesis, Maastricht University; with summary in Dutch ISBN 9090161643

Cover design \& layout: Patrick Rump Production: Stichting Drukkerij C. Regenboog, Groningen 


\title{
Polyunsaturated fatty acids and the fetal origins hypothesis
}

\author{
A study on cardiovascular risk indicators in children
}

\section{PROEFSCHRIFT}

ter verkrijging van de graad van doctor aan de Universiteit Maastricht, op gezag van de Rector Magnificus, Prof. dr A.C. Nieuwenhuijzen Kruseman, volgens het besluit van het College van Decanen, in het openbaar te verdedigen op

vrijdag 6 december 2002 om 14:00 uur

door

\section{Patrick Rump}

geboren op 25 september 1967 te Sliedrecht 


\section{Promotores}

Prof. dr G. Hornstra

Prof. dr ir R.P. Mensink

\section{Beoordelingscommissie}

Prof. dr E. Mariman (voorzitter)

Prof. dr T.W.A. de Bruin

Dr J.H.P. van der Meulen (London School of Hygiene \& Tropical Medicine)

Prof. dr F.A.J. Muskiet (Rijksuniversiteit Groningen)

Prof. dr ir W.H.M. Saris

\section{ilứlin}

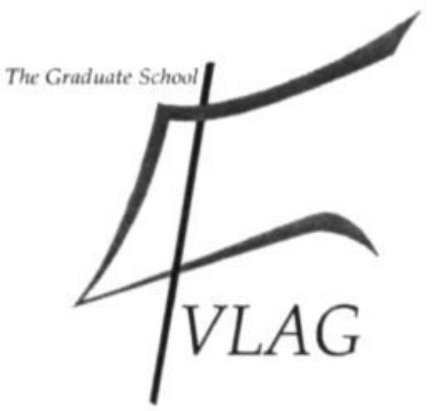

The study presented in this thesis was part of the 'fatty acid follow-up study', performed at the Nutrition and Toxicology Research Institute Maastricht (NUTRIM), which participates in the graduate school Food Technology, Agrobiotechnology, Nutrition and Health Sciences (VLAG-II) accredited by the Royal Netherlands Academy of Arts and Sciences (KNAW).

The study described in this thesis was financially supported by the Dutch Organization for Scientific Research (NWO, grant number 90462 186) and by the University Hospital of Maastricht (Profilerings Fonds). The blinded fatty acid analyses were financed by Royal Numico, Wageningen.

Financial support by the Netherlands Heart Foundation for the publication of this thesis is gratefully acknowledged. 
De band, die 't harte bindt Der moeder aan het kind, Gebaard met wee en smarte. Aan hare borst met melk gevoed. Zo lang gedragen onder 't harte, Verbint het bloed.

Joost van den Vondel, 1637 


\section{Contents | Polyunsaturated fatty acids and the fetal origins hypothesis}

Chapter 1

Chapter 2

Chapter 3

Chapter 4

Chapter 5

Chapter 6

Chapter 7
General introduction $\mid 9$

The $\mathrm{n}-3$ and $\mathrm{n}-6$ polyunsaturated fatty acid composition of plasma phospholipids in pregnant women and their infants. Relationship with maternal linoleic acid intake | 39

Essential fatty acid composition of plasma phospholipids and birth weight: a study in term neonates $\mid 55$

Components of the insulin resistance syndrome in seven-year-old children: relations with birth weight and the polyunsaturated fatty acid content of umbilical cord plasma phospholipids | 75

Fasting triacylglycerol concentrations in seven-year-old children are related to the polyunsaturated fatty acid composition of umbilical cord plasma phospholipids | 89

Interaction between a common variant of the cholesteryl ester transfer protein gene and the apolipoprotein $\mathrm{E}$ polymorphism: effects on plasma lipids and lipoproteins in a cohort of seven-year-old children | 103

General discussion $\mid 117$

Abbreviations | 139

Summary $\mid 141$

Samenvatting | 147

Dankwoord | 153

Curriculum vitae $\mid 155$

Publications | 157

Appendices | 159 

Chapter 1 | General introduction 


\section{Chapter 1}

\section{Cardiovascular diseases in the Netherlands}

Cardiovascular diseases, including ischemic heart disease and cerebrovascular diseases, are responsible for about $30 \%$ of all deaths worldwide. ${ }^{1}$ Together they have become the leading causes of death, surpassing infectious diseases and cancer. In the United States of America 724,859 people died as a consequence of heart diseases in $1998 .{ }^{2}$ Cerebrovascular diseases accounted for 158,448 deaths. In the Netherlands approximately $37 \%$ of all deaths are caused by cardiovascular diseases, accounting for the death of 50,545 people in $1997 .{ }^{3}$ Within Europe, cardiovascular disease mortality rates differ substantially between countries (Figure 1.1). In general, the age-adjusted mortality rates from ischemic heart disease are lower in Mediterranean countries than observed in Northern European countries.

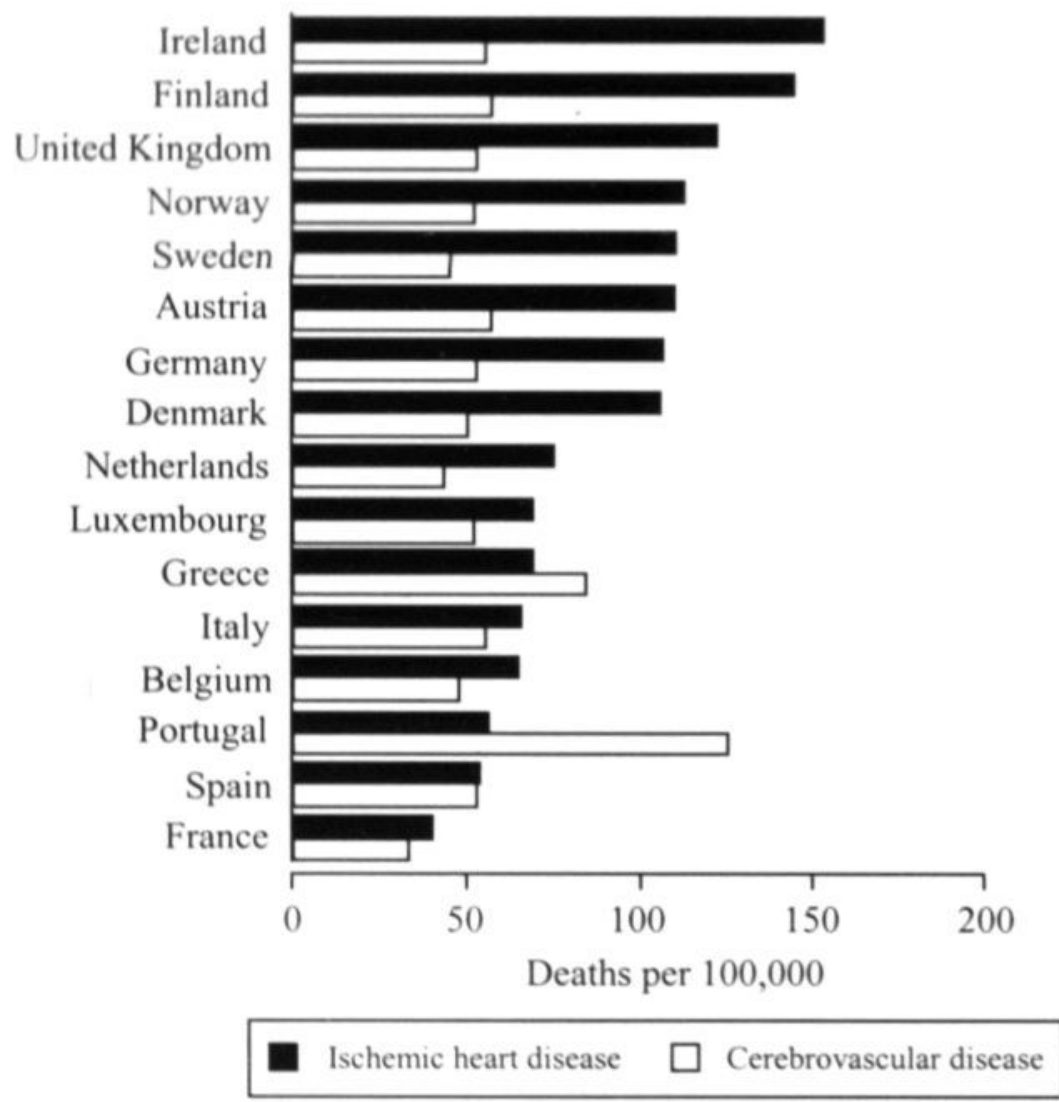

Figure 1.1: Age-standardized death rates for ischemic heart disease and cerebrovascular diseases in a selection of European countries. Death rates are given per 100,000 of the World Health Organization standard population. ${ }^{4}$ 
Although the age-adjusted mortality rates for cardiovascular diseases are declining in the Netherlands, ${ }^{3,5}$ cardiovascular diseases still remain among the largest of all medical problems. The number of Dutch patients admitted to a hospital because of cardiovascular diseases increased by $53 \%$ between 1972 and $1997 .{ }^{3}$ This increase was mainly caused by the increased admittance of older subjects (aged 65 years or over). Due to the improvement of medical care, patients with cardiovascular diseases live longer. But since these patients have an increased risk for recurrent atherosclerotic events, heart failure and other complications, the need for medical treatment increases. The progress of medical science has also led to higher age-limits for advanced treatments. As a consequence, the number of interventions and the costs per intervention increases. In 1994, the total costs related to cardiovascular diseases in the Netherlands has been valued at $€ 2.8$ billion and was only exceeded by the costs for psychological disorders. $^{6}$ In the period between 1988 and 1994, the costs for cardiovascular diseases had increased by approximately seven percent each year. Even higher increases of the costs are expected for the future. ${ }^{6}$ These figures illustrate the large economic and medical impact of cardiovascular diseases in the Netherlands and emphasize the need to continue the search for preventive measures.

\section{On the childhood origins of cardiovascular diseases}

\section{Cardiovascular risk indicators}

During the past century, scientific research has contributed substantially to our understanding on the etiology of cardiovascular diseases and the underlying processes of inflammation, atherosclerosis and thrombosis. ${ }^{7,8}$ Prospective population surveys, such as the Framingham Heart Study, ${ }^{9}$ the Northwick Park Heart Study ${ }^{10}$ or the Seven Countries Study, ${ }^{11}$ disclosed the most important characteristics related to an increased risk for the development of cardiovascular diseases. Among the principal risk indicators identified are a high serum cholesterol concentration, ${ }^{12-15}$ hypertension, ${ }^{16-19}$ and smoking. ${ }^{20-22}$ Additional risk variables are diabetes, ${ }^{23-25}$ obesity, ${ }^{26,27}$ elevated plasma triacylglycerol levels, ${ }^{28-32}$ a sedentary life style ${ }^{33-35}$ and a positive family history of early atherosclerotic events. ${ }^{36-38}$ A substantial number of other indicators have been identified. A survey conducted by Hopkins and Williams resulted in a list of 246 potential coronary risk variables. ${ }^{39}$ More recently, biochemical markers for inflammation such as C-reactive protein (CRP) were added to this long list. ${ }^{40,41}$

It is believed that combined - acting simultaneously or sequentially - such environmental and genetic factors may contribute in a synergistic way to the development of cardiovascular diseases. Many of these characteristics are already present in adolescents and children and it has been suggested that subjects at risk for the development of cardiovascular diseases can be identified at a young age. ${ }^{42,43}$ 


\section{Clustering of cardiovascular risk indicators}

Cardiovascular risk indicators are not independent entities. They tend to cluster in certain individuals. In adults, the combination of hyperinsulinemia, hypercholesterolemia, hypertension and coronary artery disease - first described by Reaven ${ }^{44}$ - is well recognized and has become known as "Syndrome X", "the metabolic syndrome" or "the insulin resistance syndrome". ${ }^{45-47}$ Obesity is often considered a component of this syndrome, ${ }^{48-50}$ but the clustering of cardiovascular risk indicators is observed in the non-obese as well. A similar clustering of cardiovascular risk variables is also observed in children and adolescents. ${ }^{51-53}$ It is believed that the development of insulin resistance plays a central role and contributes to the clustering of the other cardiovascular risk indicators. ${ }^{54,55}$

\section{Cardiovascular risk indicators and the childhood origin of atherosclerosis}

One of the most important processes related to the development of cardiovascular diseases is atherosclerosis. Apart from relatively rare cases suffering from genetic disorders like familial hypercholesterolaemia, clinically relevant or symptomatic atherosclerotic lesions are generally not observed at a pediatric age. However, evidence from autopsy studies suggests that the subclinical development of atherosclerosis start during childhood already. ${ }^{56,57}$ In young adults and children, early stages of the atherosclerotic process (i.e. fatty streaks, intermediate lesions, fibrous plaques or even more advanced lesions) can be found in the aorta and coronary arteries. ${ }^{58-61}$ In a study based on more than 500 autopsies, approximately one third of the deceased children under 9 years of age had simple intimal fatty streaks in the left coronary artery. ${ }^{62}$ At puberty, more than $50 \%$ had larger lesions containing foam cells, extracellular lipid, and lipid in smooth muscle cells. By the age of 20 to 30 years, one third of young adults had developed raised lesions with fibromuscular caps and extracellular lipid cores. The size and number of lesions like these increases with age and are associated with the ante-mortem presence of several cardiovascular risk indicators. ${ }^{63-70}$ Combinations of obesity, high blood pressure, elevated serum concentrations of triacylglycerol, total cholesterol and low-density lipoprotein cholesterol (LDL-cholesterol), or low serum concentrations of high-density lipoprotein cholesterol (HDL-cholesterol), during childhood are all related to the extent of the atherosclerotic lesions present. ${ }^{64,67,69}$ These findings demonstrate an association between cardiovascular risk indicators and the development of atherosclerosis in the young, and they seem to justify the evaluation of such risk variables in children.

\section{Tracking of cardiovascular risk indicators from childhood into adulthood}

The development of the multi-factorial hypothesis for the origins of cardiovascular diseases and the recognition that atherosclerosis starts at a young age already, prompted researchers to study cardiovascular risk indicators in children during the 1960 s and 1970 s. $^{71}$ Since that time, numerous studies have contributed to the currently available 
information on risk variables in children and on the trends of such variables over time. $^{51,72-77}$ An important observation was that children tend to maintain their ranking order with respect to several cardiovascular risk indicators from childhood into adulthood; a phenomenon referred to as "tracking". Tracking has been demonstrated for serum lipid and lipoprotein concentrations, ${ }^{78,79}$ obesity, ${ }^{80-82}$ blood pressure, ${ }^{83,84}$ fitness (aerobic power), ${ }^{85}$ and plasma insulin concentration. ${ }^{86,87}$ The occurrence of tracking implies that the evaluation of cardiovascular risk indicators in children can indeed be used to identify groups of individuals at potential risk for developing cardiovascular diseases later in life. In this light, the recently reported increases in the prevalence of obesity and non-insulin-dependent diabetes mellitus among children and adolescents certainly justify some concerns for the future. ${ }^{88-91}$

\section{On the fetal origins of cardiovascular diseases}

Aside from the classical risk variables discussed above, a new and intriguing risk indicator has been identified during recent years. It suggests that adult cardiovascular diseases may not only arise from the prolonged effects of genetic and environmental factors acting since childhood but could also in part originate from experiences during the short period of life before birth. A small size at birth (or during infancy) has namely been associated to an increased risk for the development of adult heart disease, Type II (non-insulin-dependent) diabetes mellitus and related risk factors such as hypertension and dyslipidemia. ${ }^{92-96}$ Different research groups have found similar relations in both men and women from various populations. ${ }^{97-104}$ These findings started a whole new field of research on the origins of cardiovascular diseases.

\section{The fetal origins hypothesis}

The associations found between infant size at birth and adult diseases led to the formulation of the so-called "fetal origins hypothesis" or the "Barker hypothesis", named after the man who revived the concept that experiences before birth contribute to the development of adult diseases. ${ }^{105}$ According to this hypothesis, the propensity to develop coronary heart disease, Type II diabetes mellitus, hypertension and other disorders are consequences of intra-uterine adaptations to fetal undernourishment. ${ }^{106}$ These adaptations are presumed to persist during adult life and considered detrimental in the postnatal abundance of food. ${ }^{107}$ The risk for adult diseases may thus be "programmed" or "imprinted" by the environment in utero. ${ }^{108.109}$ Studies on subjects born around the time of the Dutch famine in the winter of 1944-1945 seem to support this concept. Prenatal exposure to the famine was associated with a higher risk for obesity, glucose intolerance and a more atherogenic lipid profile in adult life. ${ }^{110-113}$ The prevalence of coronary heart disease was higher in people prenatally exposed to the Dutch famine (during early gestation only) compared to those born before or after the famine. ${ }^{114}$ A similar study on subjects born during the Leningrad siege between 1941 
and 1944 did not reveal such associations. ${ }^{115}$ The comparability of the conditions before, during and after the Leningrad siege and the Dutch famine are, however, debated. ${ }^{113}$

\section{Evidence for the fetal origins hypothesis in children}

According to Whincup, studies on the fetal origins of cardiovascular diseases in children are important since they may complement the epidemiological studies in adults in several ways. ${ }^{116}$ First, potential confounding factors such as adult life style (e.g. smoking, alcohol use or certain dietary habits) are hardly operant during childhood. Secondly, the effects of the fetal environment may interact with postnatal factors such as growth, especially the development of obesity; ${ }^{98,99}$ studies in children allow us to evaluate these interactive effects. Additionally, most studies in adults are based on prewar birth cohorts; studies in children (born under current perinatal circumstances) further allow us to examine the temporary relevance of the fetal origins hypothesis. ${ }^{116}$

In children, birth weight or other measures of size at birth have indeed been related to a diversity of cardiovascular risk indicators. Negative relations between size at birth and systolic blood pressure have been reported in many birth cohorts, ${ }^{117-122}$ including a cohort of Dutch children. ${ }^{123}$ This association appears to be dependent on current body weight, body mass index or on other parameters of postnatal growth. It further seems that the relation between small size at birth and childhood blood pressure becomes stronger with increasing age but may be attenuated during puberty. ${ }^{117,119,124,125}$ Indices of glycaemic control (such as fasting insulin or pro-insulin concentration, glycated hemoglobin level or glucose intolerance) in children have also been associated to a small size at birth. ${ }^{120,126,127}$ Moreover, serum concentrations of cholesterol and triacylglycerol were negatively related to size at birth in some of the studied cohorts. ${ }^{120,126,128}$ A lower weight at birth has also been related to (central) adiposity in children, ${ }^{126,129-131}$ but this relation with body fatness has been refuted in other studies. ${ }^{132-134}$ The reported associations between small size at birth and cardiovascular risk indicators were not found in all populations studied. ${ }^{135,136}$ In some studies, the associations found did not seem to indicate a relation with impaired fetal nutrition (e.g. as reflected by a low ponderal index). ${ }^{121,135}$

Postnatal "catch-up" growth, the return to the genetically determined growth trajectory after birth, might be a more sensitive indicator of intra-uterine growth restraint caused by environmental factors than a small size at birth itself. Postnatal "catch-up" growth has been associated to an increased body weight, body mass index, percent body fat, and waist circumference in British five-year-olds. ${ }^{137}$ These variables predict adult obesity and are related to a variety of cardiovascular risk indicators in children. ${ }^{81,138}$ Moreover, postnatal "catch-up" growth has indeed been associated to adult coronary heart disease mortality. ${ }^{104,139}$ The combination of a small size at birth and subsequent development of obesity during childhood thus seems to be of particular 
importance for the development of an unfavorable cardiovascular risk profile. ${ }^{116,126,139,140}$

\section{Suggested mechanisms to explain the fetal origins of adult diseases}

A diversity of mechanisms have been proposed to explain how intra-uterine adaptations to fetal undernourishment could eventually lead to the development of adult diseases. ${ }^{106}$ Fetal growth is characterized by periods of rapid cell division and different tissues grow during various so-called "critical periods". 141 The availability of oxygen and nutrients during such periods of cell division may influence the rate of growth and permanently determine the number of cells in particular organs. ${ }^{106}$ In animal experiments, periods of undernourishment have been related to reduced numbers of cells in various tissues. $^{142,143}$ Furthermore, such alterations in the diet (e.g. low protein intake) of pregnant animals have been demonstrated to cause persisting changes in the metabolism of their offspring. ${ }^{144,145}$ In humans, a reduction of the number of pancreatic $\beta$-cells or renal nephrons at birth have been suggested to explain the increased risk for the development of Type II diabetes mellitus or hypertension later in life. ${ }^{146,147}$

Alternatively, nutritional factors may permanently alter gene expression in certain tissues or could affect the selection of cell lines that will ultimately form a particular organ (clonal selection). ${ }^{108}$ The effects of nutrients are not necessarily direct and on a cellular level; it is very well possible that they could be mediated through various hormone systems (e.g. insulin, glucocorticoids, gut hormones etc.). ${ }^{108,148-150}$ Several epigenetic mechanisms have also been proposed, including the possibility of genomic imprinting in response to environmental factors such as nutrition during development. ${ }^{151.152}$ Others suggest that the associations found between infant size at birth and adult diseases, especially Type II diabetes mellitus, can readily be explained by common genetic variations rather than by fetal nutrition. ${ }^{153-155}$ Insulin plays a key role in fetal growth, and a disturbed insulin metabolism caused by such genetic factors could be responsible for both a low infant weight at birth and the propensity to develop Type II diabetes mellitus later in life.

In short, a large diversity of mechanisms have been proposed to explain the relations between a small size at birth and adult diseases but scarce evidence for each is available. At present, it seems unlikely that only one factor (one gene, an enzyme, a hormone, one specific nutrient) or mechanism can explain all of the observed phenomena. $^{156}$

\section{Essential fatty acids and the early origins of cardiovascular diseases}

The suggestion that nutrition plays a central role in the fetal origins of adult diseases is now supported by three sets of evidence. ${ }^{157}$ First, support comes from the epidemiological findings of the Dutch Hunger Winter studies (discussed above). ${ }^{110-113}$ Secondly, manipulation of the maternal diet in pregnant animals permanently alters the 
physiology of their offspring in a way that is consistent with the phenomena observed in human epidemiological studies. ${ }^{144,158-161}$ And finally, the third line of evidence comes from the current knowledge of the regulation of fetal growth, in which the fetal supply of nutrients play a key role. ${ }^{157,162}$

So far, only a very small number of human studies have been conducted to identify the nutrients involved. Those who did mostly focussed on the effects of the balance of macro-nutrients (e.g. the protein/carbohydrate balance) of the maternal diet during pregnancy. ${ }^{163-165}$ Hardly any work has been done to explore the importance of specific nutrients and their balance on fetal growth and later outcome. ${ }^{166}$ For instance, based on animal experiments the availability of specific amino acids, such as taurine, may have critical roles. ${ }^{167}$ Other specific nutrients that have been implicated, but have not been studied in this context so far, are essential fatty acids and their derivatives. ${ }^{168-}$ ${ }^{170}$ Beneficial effects of dietary essential fatty acids have been shown in relation to adult cardiovascular diseases and related disorders like dyslipidemia, hypertension, thrombosis, and insulin resistance. ${ }^{171-183}$ Whether the fetal availability of these substances may influence later cardiovascular risk is unknown. But recent findings from animal experiments certainly seem to support the idea that the availability of specific fatty acids early in life may be of importance. ${ }^{184}$

\section{Essential fatty acids and their derivatives}

At the start of the previous century, Burr and Burr discovered that a fat-free diet causes retarded growth, scaly dermatitis, trans-epidermal water loss, renal disorders, reproductive failure and even death in rats. ${ }^{185,186}$ Subsequent supple-mentation with small amounts of vegetable oils markedly improved the deficiency symptoms. ${ }^{185,186}$ Their findings clearly demonstrated that certain fatty acids are essential constituents of the diet in animals. Several decades later, two essential fatty acids (i.e. linoleic acid and $\alpha$-linolenic acid) were identified and proven to be indispensable for humans as well. ${ }^{187 .}$ ${ }^{191}$ Since man lack the required enzymes for the synthesis of linoleic acid and $\alpha$ linolenic acid, these fatty acids have to be obtained through the diet. Once consumed, linoleic acid and $\alpha$-linolenic acid can be converted into a diversity of products with important biological functions (Figure 1.2). Because there is no interconversion between the products derived from linoleic acid and those derived from $\alpha$-linolenic acid, the derivatives are either designated to the so-called $n-3$ (or $\omega-3$ ) family (derived from $\alpha$-linolenic acid) or to the $n-6$ (or $\omega-6$ ) family (derived from linoleic acid) of fatty acids.

The $n-3$ and $n-6$ fatty acids are found especially in (membrane) phospholipids. They are not just a source of energy, but are also necessary for maintaining particular bodily functions. Linoleic acid is important for maintaining the integrity of the epidermal water barrier ${ }^{192,193}$ and is also the precursor for dihomo- $\gamma$-linolenic acid and arachidonic acid, which are both substrates for the production of eicosanoids from the 1 - and 2-series, respectively. ${ }^{194,195}$ Eicosapentaenoic acid is a derivative of $\alpha$-linolenic 
acid, and is the precursor for the 3-series eicosanoids. These eicosanoids are active mediators of many physiological and pathological processes like, for instance, immunological responses, blood pressure regulation, reproduction, body temperature regulation, and blood coagulation. ${ }^{196,197}$ Additionally, arachidonic acid and docosahexaenoic acid are important structural cellular components in the brain, and are believed to fulfill an array of membrane associated functions. These may include alterations in cellular ion transport, hormone receptor number or affinity, signal transduction and membrane fluidity. ${ }^{198-201}$ Moreover, essential fatty acids and their products may regulate gene expression, directly or via regulatory proteins such as the so-called peroxisome proliferator-activated receptors (PPARs). ${ }^{202-204}$

When the availability of essential fatty acids or their products is lower than required, the human body starts to synthesize fatty acids of similar molecular structure. One example is eicosatrienoic acid (Mead acid; 20:3n-9), which is a derivative of oleic acid $(18: \ln -9) .{ }^{205}$ Eicosatrienoic acid is therefore regarded as a marker for an essential fatty acid shortage. Another example is docosapentaenoic acid (Osbond acid; 22:5n-6), which is considered a marker for a limited availability of especially docosahexaenoic acid. ${ }^{206}$ Relatively high amounts of these fatty acids have been found in fetal blood and tissue. ${ }^{207,208}$ This finding has led to the suggestion that current fetal essential fatty acid availability might not be adequate, or at least, seems marginal. ${ }^{208,209}$

\section{Fetal essential fatty acid availability}

Because the fetus can not synthesize linoleic acid and $\alpha$-linolenic acid, these fatty acids have to be obtained from the maternal circulation. The source of the derived products is less well known. They may be synthesized by the placenta or by the fetus itself. However, since the fetal and placental capacity to do so seems rather low, ${ }^{210-213}$ it is more likely that these products are also derived from the maternal circulation by placental transfer. The close relationship found between maternal dietary fatty acid intake and blood fatty acid concentrations on one hand and fetal blood fatty acid concentrations on the other hand, support this notion. ${ }^{214-216}$ Fatty acid transport across the placenta might be selective. .17-219 $^{2}$

When comparing fetal blood concentrations of fatty acids with maternal blood concentrations (expressed as \% by wt of total fatty acids), higher concentrations of long-chain poly-unsaturated fatty acids and lower concentrations of the essential fatty acids linoleic acid and $\alpha$-linolenic acid are found in fetal blood. ${ }^{214,220,221}$ The concentrations ( $\%$ by wt of total fatty acids) of long-chain polyunsaturated fatty acids of the $\mathrm{n}-3$ and $\mathrm{n}-6$ family also increases from maternal blood, placenta, fetal blood, fetal liver and finally fetal brain. ${ }^{222}$ These observations suggest a preferred transfer of the long-chain polyunsaturated derivatives of linoleic acid and $\alpha$-linolenic acid to fetal tissues. The term "biomagnification" has been introduced to describe this phenomenon. ${ }^{222}$ 
Based on blood and tissue concentrations, the quantitative accretion of n-3 and n-6 long-chain polyunsaturated fatty acids by the fetus increases progressively during gestation. ${ }^{223-226}$ The last trimester of pregnancy is therefore regarded as an important period for fetal fatty acid supply. Preterm infants are devoid of this important phase in life and are born with lower essential fatty acid concentrations in their blood and tissues than infants carried to term. ${ }^{227}$

Infants born with a lower weight at birth also have lower blood and tissue essential fatty acid concentrations. ${ }^{228}$ Considering that growth retardation is one of the prominent features of essential fatty acid deficiency, ${ }^{185-187,190}$ the availability of essential fatty acids might be a determinant of fetal growth. In premature infants, birth weight was positively related to the proportions of arachidonic acid, dihomo- $\gamma$-linolenic acid and docosahexaenoic acid measured in blood or tissue lipid fractions. ${ }^{229-233}$ Moreover, higher blood and tissue concentrations of essential fatty acid deficiency markers (like Mead acid) have been found in low-birth-weight neonates. ${ }^{228}$ Such findings suggest an important role for essential fatty acids in fetal growth. ${ }^{234} \mathrm{~A}$ few studies have indicated that the maternal intake of specific fatty acids (particularly n-3 fatty acids) might influence the length of gestation and thereby infant weight at birth. ${ }^{235,236}$ More recent work has, however, largely failed to confirm that birth weight might be influenced in such a way. ${ }^{237}$

The combination of the suggested inadequacy of fetal essential fatty acid availability under current dietary conditions, the potential importance of essential fatty acids for fetal growth, and the wide array of regulatory functions of essential fatty acids and their products, renders them as attractive candidate nutrients involved in the fetal origins of cardiovascular diseases.

\section{Fetal versus maternal nutrition}

The availability of nutrients to the fetus is ultimately dependent upon the diet of its mother, but factors like maternal metabolism, uterine and umbilical blood flows, and placental transfer and metabolism play a very important role as well. ${ }^{157,162}$ The way in which the placenta is able to deliver the required substances will eventually determine the nutritional status of the developing fetus. For this reason, measuring maternal nutrient intake or nutritional status (e.g. using biochemical indicators) does not necessarily provide an accurate estimate of fetal nutrient availability. This aspect may in part explain why the influence of maternal nutrition on infant size at birth seems absent or small in many human studies. ${ }^{238-241}$ Fetal nutrition should thus be distinguished from maternal nutrition. ${ }^{157}$ The fetal availability of specific nutrients like fatty acids might thus be studied at best by using fetal biochemical indicators such as measurements of neonatal blood or tissue fatty acid concentrations. 


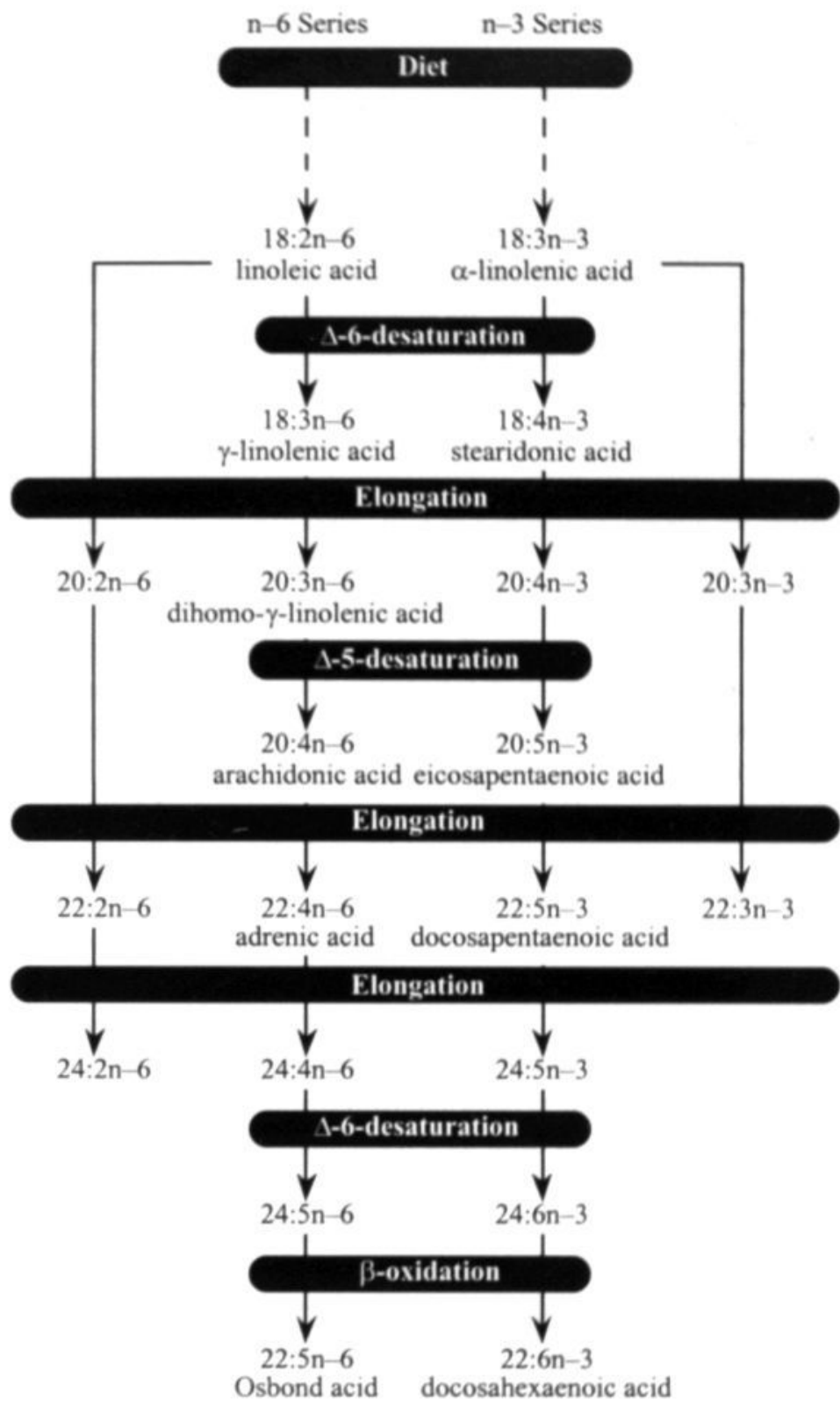

Figure 1.2: Main pathways for the conversion of the essential fatty acids linoleic acid $(18: 2 n-6)$ and $\alpha$ linolenic acid $(18: 3 n-3)$ into their longer-chain polyunsaturated derivatives. The nomenclature used for fatty acids is as follows: the number of carbon atoms, followed by a colon and the number of double bonds; the fatty acid family is indicated by designation of the first double bond from the methyl terminus of the carbon chain. 


\section{Common genetic variations}

The risk for the development of cardiovascular diseases is in part determined by hereditary factors. ${ }^{242,243}$ Numerous rare and common genetic variations have now been identified and studied in relation to cardiovascular diseases, non-insulin-dependent diabetes mellitus, obesity, dyslipidemia, hypertension or serum concentrations of fibrinolytic or coagulation factors. Well-known are the mutations in the LDL-receptor gene, leading to familial hypercholesterolaemia. These mutations are found in approximately 1 out of $400-500$ individuals. Relatively rare mutations like these, however, will contribute little to the total variation of serum lipid and lipoprotein concentrations observed within a population. Other mutations, found at a much higher rate in the general population are probably of more importance. A few well-known common variants (so-called "polymorphisms") are discussed here in more detail.

Hydrophobic molecules such as cholesterol, triacylglycerol and other lipids are transported in the aqueous surroundings of the blood by circulating lipoprotein particles. These lipoprotein particles contain so-called apolipoproteins, the protein moieties of these particles. Common variations in the genes coding for these apolipoproteins may affect serum concentrations of lipoproteins (like LDL or HDL) and thereby influence the risk for cardiovascular diseases. ${ }^{244.245}$ Apolipoprotein E (apo E), for instance, is present in both apolipoprotein B-containing particles (such as LDL) as well as apolipoprotein Al-containing lipoproteins (i.e. HDL). It has been estimated that genetic variation at the apo E locus may account for up to $10-15 \%$ of the observed inter-individual variation in serum total cholesterol and LDL-cholesterol concentrations. ${ }^{246-248}$

The most studied polymorphism of apo $\mathrm{E}$ is the combination of variations in amino acids at residues 112 and 158 . The $\varepsilon 2$ allele (substitution of arginine at position 158 by cysteine) is associated with lower concentrations of serum total cholesterol and LDL-cholesterol, whereas the $\varepsilon 4$ allele (substitution of cysteine at position 112 by arginine) relates to higher levels of total cholesterol and LDL-cholesterol. ${ }^{249,250}$ These effects of apo E genotype are observed at a paediatric age already, ${ }^{251}$ as well as during infancy. ${ }^{252}$ Variations at the apo E locus have also been shown to both influence the clustering of cardiovascular risk indicators in children ${ }^{253}$ and the tracking of cholesterol and lipoprotein levels from childhood into adulthood. ${ }^{254-257}$ Moreover, genetic variation at the apo E locus in children and young adults is related to the parental history of myocardial infarction. ${ }^{253.258}$ Similarly, genetic variations in other apolipoproteins (e.g. apo B, apo A-I or apo CIII) may also affect serum concentrations of lipids and lipoproteins in children and thereby contribute to the risk of heart disease in adult life. $^{259}$

Apart from variations in genes coding for apolipoproteins, variations in other genes like those coding for enzymes involved in lipid and lipoprotein metabolism are important as well. One example is the enzyme called cholesteryl ester transfer protein (CETP). ${ }^{260}$ CETP plays a key role in reverse cholesterol transport; the transport of cell- 
derived cholesterol back to the liver mediated by HDL particles. It mediates the transfer of cholesteryl esters from HDL to apo B containing lipoproteins like LDL and verylow-density lipoprotein (VLDL), in exchange for triacylglycerol. ${ }^{261}$ An increased CETP activity is negatively related to serum levels of HDL cholesterol and positively related to VLDL and LDL cholesterol concentrations. ${ }^{261}$ In adults, plasma CETP concentrations, CETP activity and HDL cholesterol levels have been associated with common variations in the CETP gene, like the Taq IB polymorphism. ${ }^{262-264}$ The presence of this polymorphism (i.e. $\mathrm{B} 1$ allele) has also been related to the progression of coronary atherosclerosis. ${ }^{265}$

Common genetic variations like these may not only be important predictors of circulating cardiovascular risk factor levels in children (and thus as co-variables), they could also influence the individual response of a subject to an environmental stimulus. Like for instance the response to dietary interventions, medical treatments, alcohol consumption or smoking. ${ }^{265-272}$ Recently, the effects of the $\varepsilon 2$ and $\varepsilon 4$ alleles at the apo E locus on serum LDL-cholesterol concentration were found to be more pronounced in adults with a lower body weight during infancy compared to adults with a higher body weight during infancy. ${ }^{273}$ The hypothesized effects of fetal essential fatty acid availability on the cardiovascular risk profile later in life may depend upon the presence of such common genetic variations.

\section{Outline of this thesis}

\section{Objectives of the study}

The primary objective of the study described in this thesis was to investigate whether the availability of essential fatty acids and their long-chain polyunsaturated derivatives during fetal live, as is reflected by the fatty acid composition of umbilical cord plasma phospholipids, relates to the cardiovascular risk profile of children. Secondly, to study associations and possible interactions with a selection of common gene variants. For this purpose cardiovascular risk indicators and gene polymorphisms were studied in a cohort of Dutch seven-year-olds.

\section{Study population}

Subjects were recruited from a birth-cohort of children. Between 1989 and 1995, pregnant women from the Maastricht region were asked to participate in a longitudinal observational study investigating the changes in essential fatty acid status during pregnancy and their relation to pregnancy outcome. ${ }^{215,216,274.275}$ Three antenatal clinics participated: the University Hospital in Maastricht, Hospital "De Wever" in Heerlen, and the School for Midwifery in Kerkrade. These centers are all located in the province of Limburg in the southern part of the Netherlands. Selection criteria for entering the study were a gestational age of less than 16 weeks at entry and no apparent sign of any cardiovascular, neurological, renal, or metabolic disorder at the time of recruitment. In 
order to determine the changes in essential fatty acid status during pregnancy, maternal blood samples were collected during the first, second and third trimester of pregnancy, and shortly after delivery. Directly after parturition, an additional blood sample was obtained from the umbilical vein to estimate fetal essential fatty acid availability. Local hospital staff members recorded individual maternal and infant characteristics on standardized data sheets. Additional information was retrieved from questionnaires and medical records. In a sub-sample of these women, the habitual maternal dietary fatty acid intake was assessed. ${ }^{215,276}$ This unique cohort of women and children provides an excellent opportunity to study potential relations between fetal essential fatty acid availability and cardiovascular risk later in life. Therefore, a follow-up study was performed between 1997 and 2000. All singleton babies born alive before 1994 - and of whom an umbilical blood sample was available for the determination of the essential fatty acid status at birth - were candidates to be included. In total, 750 children were eligible for follow-up.

\section{Participation during the follow-up study}

With the aid of the Dutch population registry $97 \%$ of the eligible population could be traced (Figure 1.3). Three of the children had died and 34 lived abroad. The parents of the remaining 691 children received an invitation for the follow-up evaluation. Despite repeated invitations, 133 parents did not respond. Eventually we were able to contact the parents of 558 children. Of these, 231 refused to participate. The most important reasons for not participating were: lack of time or interest $(39 \%)$, medical problems of the child $(12 \%)$, medical problems of another family member $(3 \%)$, burden of the protocol $(12 \%)$, travel distance or lack of transportation $(10 \%)$, the lack of a financial reward $(1 \%)$, reason unknown $(11 \%)$ or for a diversity of other reasons $(13 \%)$. In total 297 children and their parents finally showed up at the appointments made for the follow-up on cardiovascular risk profile. The birth characteristics of these 297 children were not significantly different from the 453 children who did not attend our clinic (Table 1.1), suggesting that the investigated children comprised a representative sample of the entire cohort.

\section{Measurements during the follow-up study}

The children were investigated before puberty, at approximately seven years of age. Fasting blood samples were collected for measurement of blood lipids and lipoproteins, fibrinolytic and coagulation factors, indices of glycaemic control and to determine the essential fatty acid status at seven years of age. Leukocytes were isolated to extract DNA for analysis of gene polymorphisms. Anthropometric data was collected to study relations with body size and body composition. Additionally, resting blood pressure was measured and maximal treadmill exercise performance was assessed. 


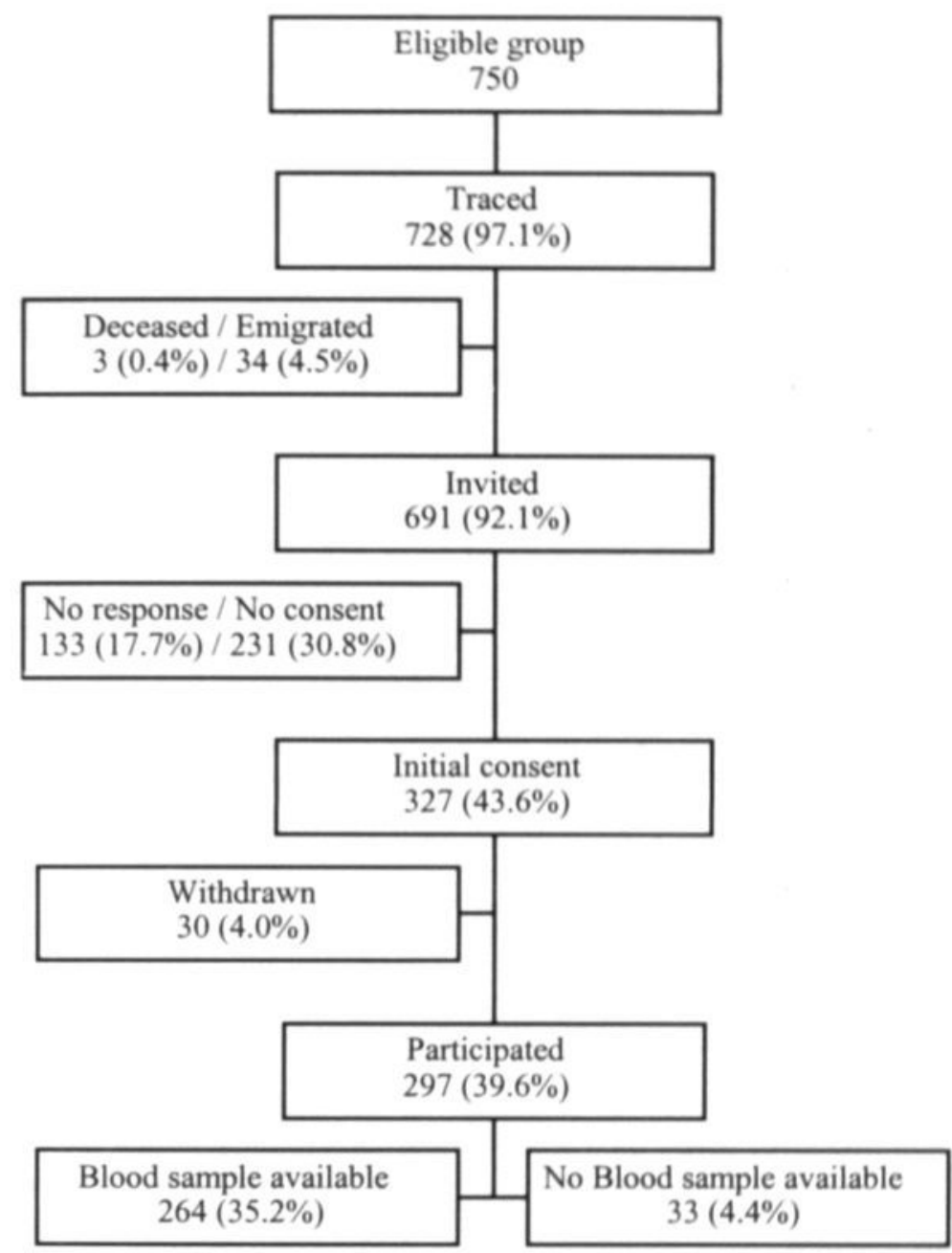

Figure 1.3: Participation during the follow-up study on cardiovascular risk profile. Values are absolute numbers or proportions of the total eligible population.

A questionnaire and a structured interview with one or both parents was used to collect additional information like socio-economic status, infant nutrition, and family history for cardiovascular diseases and related disorders. 
Table 1.1: Comparison of birth characteristics between participants and non-participants.

\begin{tabular}{lcc}
\hline Characteristic & $\begin{array}{c}\text { Participants } \\
(n=297)\end{array}$ & $\begin{array}{c}\text { Non-participants } \\
(n=453)\end{array}$ \\
\hline Sex ratio $(\mathrm{M}: \mathrm{F})$ & $164: 133$ & $254: 199$ \\
Length of gestation (wk) & $39.9(39.7 ; 40.1)$ & $39.9(39.7 ; 40.0)$ \\
Birth weight $(\mathrm{g})$ & $3301(3243 ; 3360)$ & $3254(3207 ; 3301)$ \\
Weight standard deviation score & $-0.13(-0.23 ;-0.02)$ & $-0.20(-0.29 ;-0.12)$ \\
Crown-heel length at birth $(\mathrm{cm})$ & $49.9(49.6 ; 50.2)$ & $49.9(49.6 ; 50.1)$ \\
Occipital-frontal circumference $(\mathrm{cm})$ & $34.4(34.2 ; 34.6)$ & $34.3(34.1 ; 34.5)$ \\
Ponderal index ${ }^{2}\left(\mathrm{~g} / \mathrm{cm}^{3}\right)$ & $2.7(2.6 ; 2.7)$ & $2.6(2.6 ; 2.7)$ \\
Born SGA & $37(12)$ & $60(13)$ \\
Born LGA & $19(6)$ & $26(6)$ \\
Mode of delivery & & \\
$\quad$ vaginal & $228(77)$ & $333(74)$ \\
$\quad$ extraction (vacuum/forceps) & $49(16)$ & $68(15)$ \\
caesarian section & $19(6)$ & $49(11)$ \\
Birth order & & $339(75)$ \\
1 & $211(71)$ & $95(21)$ \\
2 & $65(22)$ & $17(4)$ \\
3 or more & $20(7)$ &
\end{tabular}

Values are mean $(95 \% \mathrm{CI})$ or number $(\%)$. Totals may vary due to missing data.

'According to Dutch references (appropriate for length of gestation, infant sex and birth order). ${ }^{277}$ Standard deviation score (or z-score) is the distance between observed birth weight and the average birth weight of the reference population expressed in standard deviation units. ${ }^{278} \mathrm{SGA}$, small for gestational age (birth weight $\leq 10^{\text {th }}$ percentile). LGA, large for gestational age (birth weight $\geq 90^{\text {th }}$ percentile).

${ }^{2}$ Ponderal index was calculated as birth weight $(\mathrm{g})$ divided by [length $\left.(\mathrm{cm})\right]^{3} \times 100$.

No statistically significant differences were found between participants and non-participants.

\section{Presentation of the results in the present thesis}

The distribution of essential fatty acid concentrations in maternal and umbilical plasma phospholipids depends upon various maternal and infant characteristics. In Chapter 2 some of these characteristics are discussed and the distribution of the concentrations of individual $\mathrm{n}-3$ and $\mathrm{n}-6$ polyunsaturated fatty acids in maternal and umbilical cord blood samples are presented. The relations between the concentrations of polyunsaturated fatty acids in umbilical cord plasma phospholipids and infant size at birth are described in Chapter 3. Further reported are the associations between umbilical cord plasma phospholipid $\mathrm{n}-3$ and $\mathrm{n}-6$ polyunsaturated fatty acid concentrations (measured at birth) and factors related to the cardiovascular risk profile of the children (measured at follow-up). These are fasting glucose, insulin, proinsulin, and leptin concentrations (Chapter 4), body size and body composition (Chapter 4), and blood lipids and lipoproteins (Chapter 5). The influences of common gene variants on plasma lipid and lipoprotein concentrations are reported in Chapter 6. An overall discussion of the results, their implications, and potential sources of erroneous conclusions is given in Chapter 7. Several appendices were added to provide additional information. 


\section{References}

1. World Health Organization. The World Health Report 2000. Health systems: improving performance. Geneva: World Health Organization; 2000.

2. National Center for Health Statistics. Health, United States, 2000. With adolescent health chartbook. Hyattsville, Maryland: National Center for Health Statistics; 2000.

3. Konings-Dalstra JAA, Reitsma JB. Hart- en vaatziekten in Nederland 1999, cijfers over ziekte en sterfte. Den Haag: Nederlandse Hartstichting; 1999.

4. World Health Organization. World Health Statistics Annual 1997-1999 (online edition). Geneva: World Health Organization; 2000.

5. Jansen J. Hart- en vaatziekten in Nederland. Ontwikkelingen en preventiemogelijkheden. Utrecht: Rijksinstituut voor Volksgezondheid en Milieuhygiëne; 1992.

6. Polder JJ, Meerding WJ, Koopmanschap MA, Bonneux L, Maas PJvd. Kosten van ziekten in nederland 1994. Rotterdam: Instituut Maatschappelijke Gezondheidszorg; 1997.

7. Ross R. The pathogenisis of atherosclerosis: a perspective for the 1990s. Nature. 1993;362:801809.

8. Berliner JA, Navab M, Fogelman AM, Frank JS, Demer LL, Edwards PA, Watson AD, Lusis AJ. Atherosclerosis: basic mechanisms. Oxidation, inflammation, and genetics. Circulation. 1995;91:2488-2496.

9. Sytkowski PA, Kannel WB, D'Agostino RB. Changes in risk factors and the decline in mortality from cardiovascular disease. The Framingham Heart Study. N Engl J Med. 1990;322:1635-1641.

10. Meade TW, Ruddock V, Stirling V, Chakrabarti R, Miller GJ. Fibrinolytic activity, clotting factors, and long-term incidence of ischaemic heart disease in the Northwick Park Heart Study. Lancet. 1993;342:1076-1079.

11. Keys A. Seven Countries - a multivariate analysis of death and coronary heart disease. Cambridge: Harvard University Press; 1980.

12. Anderson KM, Castelli WP, Levy D. Cholesterol and mortality. 30 years of follow-up from the Framingham study. JAMA. 1987;257:2176-2180.

13. Abbott RD, Wilson PW, Kannel WB, Castelli WP. High density lipoprotein cholesterol, total cholesterol screening, and myocardial infarction. The Framingham Study. Arteriosclerosis. 1988;8:207-211.

14. Stamler J, Wentworth D, Neaton JD. Is relationship between serum cholesterol and risk of premature death from coronary heart disease continuous and graded? Findings in 356,222 primary screenees of the Multiple Risk Factor Intervention Trial (MRFIT). JAMA. 1986;256:2823-2828.

15. Wald NJ, Law MR. Serum cholesterol and ischaemic heart disease. Atherosclerosis. 1995;118:S1-S5.

16. Martin MJ, Hulley SB, Browner WS, Kuller LH, Wentworth D. Serum cholesterol, blood pressure, and mortality: implications from a cohort of 361,662 men. Lancet. 1986;2:933-936.

17. Neaton JD, Wentworth D. Serum cholesterol, blood pressure, cigarette smoking, and death from coronary heart disease. Overall findings and differences by age for 316,099 white men. Multiple Risk Factor Intervention Trial Research Group. Arch Intern Med. 1992;152:56-64.

18. Collins R, Peto R, Godwin J, MacMahon S. Blood pressure and coronary heart disease [letter]. Lancet. 1990;336:370-371.

19. Collins R, Peto R, MacMahon S, Hebert P, Fiebach NH, Eberlein KA, Godwin J, Qizilbash N, Taylor JO, Hennekens CH. Blood pressure, stroke, and coronary heart disease. Part 2, Short-term reductions in blood pressure: overview of randomised drug trials in their epidemiological context. Lancet. 1990;335:827-838. 
20. Holbrook JH, Grundy SM, Hennekens CH, Kannel WB, Strong JP. Cigarette smoking and cardiovascular diseases. A statement for health professionals by a task force appointed by the steering committee of the American Heart Association. Circulation. 1984;70:1114A-1117A.

21. Fielding JE. Smoking: health effects and control (1). N Engl J Med. 1985;313:491-498.

22. Fielding JE. Smoking: health effects and control (2). N Engl J Med. 1985;313:555-561.

23. Pyorala K, Savolainen E, Lehtovirta E, Punsar S, Siltanen P. Glucose tolerance and coronary heart disease: Helsinki Policemen Study. J Chronic Dis. 1979;32:729-745.

24. Pyorala K. Relationship of glucose tolerance and plasma insulin to the incidence of coronary heart disease: results from two population studies in Finland. Diabetes Care. 1979;2:131-141.

25. Pyorala K, Savolainen E, Kaukola S, Haapakoski J. Plasma insulin as coronary heart disease risk factor: relationship to other risk factors and predictive value during $91 / 2$-year follow-up of the Helsinki Policemen Study population. Acta Med Scand Suppl. 1985;701:38-52.

26. Larsson B, Svardsudd K, Welin L, Wilhelmsen L, Bjorntorp P, Tibblin G. Abdominal adipose tissue distribution, obesity, and risk of cardiovascular disease and death: 13 year follow up of participants in the study of men born in 1913. Br Med J Clin Res Ed. 1984;288:1401-1404.

27. Garrison RJ, Castelli WP. Weight and thirty-year mortality of men in the Framingham Study. Ann Intern Med. 1985;103:1006-1009.

28. Austin MA. Plasma triglyceride as a risk factor for coronary heart disease. The epidemiologic evidence and beyond. Am J Epidemiol. 1989;129:249-259.

29. Austin MA. Epidemiology of hypertriglyceridemia and cardiovascular disease. Am J Cardiol. 1999:83:13F-16F.

30. Castelli WP. Epidemiology of triglycerides: A view from Framingham. Am $J$ Cardiol. 1992; $70: 3 \mathrm{H}-9 \mathrm{H}$.

31. Davignon J, Cohn JS. Triglycerides: a risk factor for coronary heart disease. Atherosclerosis. 1996; 124:S57-S64.

32. Assmann G, Schulte H. Role of triglycerides in coronary artery disease: lessons from the Prospective Cardiovascular Münster Study. Am J Cardiol. 1992;70:10H-13H.

33. Paffenbarger RS, Wing AL, Hyde RT. Physical activity as an index of heart attack risk in college alumni. Am J Epidemiol. 1978;108:161-175.

34. Leon AS, Connett J, Jacobs DR, Rauramaa R. Leisure-time physical activity levels and risk of coronary heart disease and death. The Multiple Risk Factor Intervention Trial. JAMA. 1987; 258:2388-2395.

35. Leon AS, Myers MJ, Connett J. Leisure time physical activity and the 16-year risks of mortality from coronary heart disease and all-causes in the Multiple Risk Factor Intervention Trial (MRFIT). Int J Sports Med. 1997;18:S208-S215.

36. Boer JM, Feskens EJ, Verschuren WM, Seidell JC, Kromhout D. The joint impact of family history of myocardial infarction and other risk factors on 12-year coronary heart disease mortality. Epidemiology. 1999;10:767-770.

37. Barrett-Connor E, Khaw K. Family history of heart attack as an independent predictor of death due to cardiovascular disease. Circulation. 1984;69:1065-1069.

38. Khaw KT, Barrett-Connor E. Family history of stroke as an independent predictor of ischemic heart disease in men and stroke in women. Am J Epidemiol. 1986;123:59-66.

39. Hopkins PN, Williams RR. A survey of 246 suggested coronary risk factors. Atherosclerosis. 1981;40:1-52.

40. Ridker PM. High-sensitivity C-reactive protein. Potential adjunct for global risk assesment in the primary prevention of cardiovascular disease. Circulation. 2001;103:1813-1818.

41. Rifai N, Ridker PM. Proposed cardiovascular risk assesment algorithm using high-sensitivity Creactive protein and lipid screening. Clin Chem. 2001;47:28-30.

42. Strong WB. Is atherosclerosis a pediatric problem: an overview. In: Strong WB, ed. Atherosclerosis: its pediatric aspects. New York: Grune \& Stratton; 1978:1-14. 
65. McGill HC, McMahan CA, Malcom GT, Oalmann MC, Strong JP. Pathobiological Determinants of Atherosclerosis in Youth (PDAY) Research Group. Relation of glycohemoglobin and adiposity to atherosclerosis in youth. Findings from the PDAY Study. Arterioscler Thromb Vasc Biol. 1995; 15:431-440.

66. McGill HC, Strong JP, Tracy RE, McMahan CA, Oalmann MC. Pathobiological Determinants of Atherosclerosis in Youth (PDAY) Research Group. Relation of postmortum renal index of hypertension to atherosclerosis in youth. Arterioscler Thromb Vasc Biol. 1995; 15:2222-2228.

67. Pathobiological Determinants of Atherosclerosis in Youth (PDAY) Research Group. Relationship of atherosclerosis in young men to serum lipoprotein cholesterol concentrations and smoking. A preliminary report from the Pathobiological Determinants of Atherosclerosis in Youth (PDAY) Research Group. JAMA. 1990;264:3018-3024.

68. Mahoney LT, Burns TL, Stanford W, Thompson BH, Witt JD, Rost CA, Lauer RM. Coronary risk factors measured in childhood and young adult life are associated with coronary artery calcification in young adults: the Muscatine Study. J Am Coll Cardiol. 1996;27:277-284.

69. Berenson GS, Srinivasan SR, Bao W, Newman WPr, Tracy RE, Wattingney WA. Association between multiple cardiovascular risk factors and atherosclerosis in children and young adults. The Bogalusa Heart Study. N Engl J Med. 1998;338:1650-1606.

70. Stary HC. Lipid and macrophage accumilations in arteries of children and the development of atherosclerosis. Am J Clin Nutr. 2000;72:1297S-1306S.

71. Srinivasan SR, Frerichs RR, Berenson GS. Serum lipids and lipoproteins in children. In: Strong WB, ed. Atherosclerosis: its pediatric aspects. New York: Grune \& Stratton; 1978:85-110.

72. Lauer RM, Connor WE, Leaverton PE, Reiter MA, Clarke WR. Coronary heart disease risk factors in school children: the Muscatine Study. J Pediatr. 1975;86:697-706.

73. Knuiman JT, Hermus RJJ, Hautvast JGAJ. Serum total and high density lipoprotein (HDL) cholesterol concentrations in rural and urban boys from 16 countries. Atherosclerosis. 1980;36:529-537.

74. Freedman DS, Srinivasan SR, Cresanta JL, Webber LS, Berenson GS. Cardiovascular risk factors from birth to 7 years of age: the Bogalusa Heart Study. Serum lipids and lipoproteins. Pediatrics. 1987;80:789-796.

75. Lauer RM, Lee J, Clarke WR. Factors affecting the relationship between childhood and adult cholesterol levels: the Muscatine Study. Pediatrics. 1988;82:309-318.

76. Sporik R, Johnstone JH, Cogswell JJ. Longitudinal study of cholesterol values in 68 children from birth to 11 years of age. Arch Dis Child. 1991;66:134-137.

77. Porkka KVK, Viikari JSA, Rönnemmaa T, Marniemi J, Åkerblom HK. Age and gender specific serum lipid and apolipoprotein fractiles of Finnish children and young adults. The Cardiovascular Risk in Young Finns Study. Acta Pediatr. 1994;83:838-848.

78. Freedman DS, Shear CL, Srinivasan SR, Webber LS, Berenson GS. Tracking of serum lipids and lipoproteins in children over an 8-year period: the Bogalusa Heart Study. Prev Med. 1985;14:203-216.

79. Mahoney LT, Lauer RM, Lee J, Clarke WR. Factors affecting tracking of coronary heart disease risk factors in children. The Muscatine Study. Ann N Y Acad Sci. 1991;623:120-132.

80. Harsha DW, Smoak CG, Nicklas TA, Webber LS, Berenson GS. Cardiovascular risk factors from birth to 7 years of age: the Bogalusa Heart Study. Tracking of body composition variables. Pediatrics. 1987;80:779-783.

81. Whitaker RC, Wright JA, Pepe MS, Seidel KD, Dietz WH. Predicting obesity in young adulthood from childhood and parental obesity. $N$ Engl J Med. 1997;337:869-873.

82. Dietz WH. Childhood weight affects adult morbidity and mortality. J Nutr 1998;128:411S-414S.

83. Shear CL, Burke GL, Freedman DS, Berenson GS. Value of childhood blood pressure measurements and family history in predicting future blood pressure status: results from 8 years follow-up in the Bogalusa Heart Study. Pediatrics. 1986;77:862-869. 
84. Burke GL, Voors AW, Shear CL, Webber LS, Smoak CG, Cresanta JL, Berenson GS. Cardiovascular risk factors from birth to 7 years of age: the Bogalusa Heart Study. Blood pressure. Pediatrics. 1987;80:784-788.

85. Anderson LB. Tracking of risk factors for coronary heart disease from adolescence to young adulthood with special emphasis on physical activity and fitness. Danish Med Bull. 1996;43:407. 418.

86. Lauer RM. Clarke WR. Childhood risk factors for high adult blood pressure: the Muscatine Study. Pediatrics, 1989;84:633-641.

87. Bao W, Srinivasan SR, Berenson GS. Persistent elevation of plasma insulin levels is associated with increased cardiovascular risk in children and young adults: the Bogalusa Heart Study. Circulation. 1996;93:54-59.

88. Troiano RP, Flegal KM, Kuczmarski RJ, Campbell SM, Johnson CL. Overweight prevalence in trends for children and adolescents. The National Health and Nutrition Surveys 1963-1991. Arch Pediatr Adolesc Med. 1995;149:1085-1091.

89. Reilly JJ, Dorosty AR, Emmett PM. Prevalence of overweight and obesity in British children: cohort study. BMJ. 1999;319:1039.

90. Fredriks AM, van Buuren S, Wit JM, Verloove-Vanhorick SP. Body index measurements in 1996-7 compared with 1980. Arch Dis Child. 2000;82:107-112.

91. Pinhas-Hamiel O, Dolan LM, Daniels SR, Standiford D, Khoury PR, Zeitler P. Increased incidence of non-insulin-dependent diabetes mellitus among children and adolescents. $J$ Pediatr. 1996;128.

92. Barker DJP, Winter PD, Osmond C, Margetts B, Simmonds SJ. Weight in infancy and death from ischaemic heart disease. Lancet. 1989;ii:577-580.

93. Hales CN, Barker DJP, Clark PMS, Cox LJ, Fall C, Osmond C, Winter PD. Fetal and infant growth and impared glucose tolerance at age 64. BMJ. 1991;303:1019-1022.

94. Osmond C, Barker DJP, Winter PD, Fall CHD, Simmonds SJ. Early growth and death from cardiovascular disease in women. BMJ. 1993;307:1519-1524.

95. Barker DJP, Hales CN, Fall CHD, Osmond C, Phillips K, Clark PMS. Type 2 (non-insulindependent) diabetes mellitus, hypertension, and hyperlipidaemia (syndrome X): relation to reduced fetal growth. Diabetologia. 1993;36:62-67.

96. Fall $\mathrm{CH}$, Vikayakumar $\mathrm{M}$, Barker DJP, Osmond $\mathrm{C}$, Duggleby $\mathrm{S}$. Weight in infancy and prevalence of coronary heart disease in adult life. BMJ. 1995;310:17-19.

97. Frankel S, Elwood P, Sweetnam P, Yarnell J, Smith GD. Birthweight, adult risk factors and incident coronary heart disease: the Caerphilly study. Public Health. 1996;110:139-143.

98. Frankel S, Elwood P, Sweetnam P, Yarnell J, Davy-Smith G. Birth weight, body mass index in middle-age, and incident coronary heart disease. Lancet. 1996;348:1478-1480.

99. Lithell HO, McKeigue PM, Berglund L, Mohson R, Lithell UB, Leon DA. Relation of size at birth to non-insulin-dependent diabetes and insulin concentrations in men aged 50-60 years. BMJ. 1996;312:406-410.

100. Curhan GC, Willett WC, Rimm EB, Spiegelman D, Ascherio AL, Stampfer MJ. Birth weight and adult hypertension, diabetes mellitus, and obesity in US men. Circulation. 1996;94:3246-3250.

101. Leon DA, Lithell HO, Vagero D, Koupilova I, Mohsen R, Berglund L, Lithell UB, McKeigue PM. Reduced fetal growth rate and increased risk of death from ischaemic heart disease: cohort study of 15000 Swedish men and women born 1915-29. BMJ. 1998;317:241-245.

102. Yarbrough DE, BarrettConnor E, KritzSilverstein D, Wingard DL. Birth weight, adult weight, and girth as predictors of the metabolic syndrome in postmenopausal women: The Rancho Bernardo Study. Diabetes Care. 1998;21:1652-1658.

103. Rich-Edwards JW, Colditz GA, Stampfer MJ, Willett WC, Gillman MW, Hennekens CH, Speizer FE, Manson JE. Birth weight and the risk for type 2 diabetes mellitus in adult women. Ann Intern Med. 1999;130:278-284. 
104. Forsén T, Eriksson JG, Tuomilehto J, Osmond C, Barker DJP. Growth in utero and during childhood among women who develop coronary heart disease: longitudinal study. BMJ. 1999;319:1403-1407.

105. Barker DJP. Mothers, babies and health in later life. Second ed. Edinburgh: Churchill Livingstone; 1998.

106. Barker DJP. The fetal and infant origins of disease. Eur J Clin Invest. 1995;25:457-463.

107. Leon DA. Fetal growth and adult disease. Eur J Clin Nutr. 1998;52:S72-S82.

108. Lucas A. Programming by early nutrition in man. In: Bock GR, Whelan J, eds. The childhood environment and adult disease. Chichester: John Wiley and Sons; 1991:38-55.

109. O'Brien PMS, Wheeler T, Barker DJP, eds. Fetal programming: influences on development and diseases in later life. London: RCOG Press; 1999.

110. Ravelli GP, Stein ZA, Susser MW. Obesity in young men after famine exposure in utero and early pregnancy. $N$ Engl J Med. 1976;7:349-354.

111. Ravelli ACJ, Meulen JHPvd, Osmond C, Barker DJP, Bleker OP. Obesity at the age of $50 \mathrm{y}$ in men and women exposed to famine prenatally. Am J Clin Nutr. 1999;70:811-816.

112. Ravelli ACJ, Meulen JHPvd, Osmond C, Barker DJP, Bleker OP. Glucose tolerance in adults after in utero exposure to the Dutch Famine. Lancet. 1998;351:173-177.

113. Roseboom TJ, Meulen JHPvd, Osmond C, Barker DJP, Ravelli ACJ, Bleker OP. Plasma lipid profile in adults after prenatal exposure to famine. Am J Clin Nutr. 2000;72:1101-1106.

114. Roseboom TJ, Meulen JHPvd, Osmond C, Barker DJP, Ravelli ACJ, Schroeder-Tanka JM, Montfrans GAv, Michels RPJ, Bleker OP. Coronary heart disease in adults after prenatal exposure to famine. Heart. 2000;84:595-598.

115. Stanner SA, Bulmer K, Andres C, Lantseva OE, Borodina V, Poteen VV, Yudkin JS. Does malnutrition in utero determine diabetes and coronary heart disease in adulthood? Results from the Leningrad siege study, a cross sectional study. BMJ. 1997;315:1342-1349.

116. Whincup PH. Fetal origins of cardiovascular risk: evidence from studies in children. In: O'Brein PMS, Wheeler T, Barker DJP, eds. Fetal programming: influences on development and disease in later life. London: RCOG Press; 1999:30-40.

117. Williams S, St. GIM, Silva PA. Intrauterine growth retardation and blood pressure at age seven and eighteen. J Clin Epidemiol. 1992;45:1257-1263.

118. Taittonen L, Nuutinen M, Turtinen J, Uhari M. Prenatal and postnatal factors in predicting later blood pressure among children: cardiovascular risk in young Finns. Pediatr Res. 1996;40:627632.

119. Lurbe E, Redon J, Alvarez V, Durazo R, Gomez A, Tacons J, Cooper RS. Relationship between birth weight and awake blood pressure in children and adolescents in absence of intrauterine growth retardation. Am J Hypertens. 1996;9:787-794.

120. Forrester TE, Wilks RJ, Bennett FI, Simeon D, Osmond C, Allen M, Chung P, Scott P. Fetal growth and cardiovascular risk factors in Jamaican schoolchildren. BMJ. 1996;312:156-160.

121. Taylor SJC, Whincup PH, Cook DG, Papacosta O, Walker M. Size at birth and blood pressure: cross sectional study in 8-11 year old children. BMJ. 1997;314:475-480.

122. Barros FC, Victora CG. Increased blood pressure in adolescents who were small for gestational age at birth: a cohort study in Brazil. Int J Epidemiol. 1999;28:676-681.

123. Uiterwaal CSPM, Anthony S, Launer LJ, Witteman JCM, Trouwborst AMW, Hofman A, Grobbee DE. Birth weight, growth, and blood pressure. An annual follow-up study of children aged 5 through 21 years. Hypertension. 1997;30:267-271.

124. Law CM, Swiet Md, Osmond C, Fayers PM, Barker DJP, Cruddas AM, Fall CHD. Initiation of hypertension in utero and its amplification throughout life. BMJ. 1993;306:24-27.

125. Law C, Shiell AW. Is blood pressure inversely related to birth weight? The strength of evidence from a systematic review of the literature. J Hypertens. 1996;14:935-941. 
126. Bavdekar A, Yajnik CS, Fall CHD, Bapat S, Pandit AN, Deshpande V, Bhave S, Kellingray SD, Joglekar C. Insulin resistance syndrome in 8-year-old Indian children. Diabetes. 1999;48:24222429.

127. Law CM, Gordon GS, Shiell AW, Barker DJP, Hales CN. Thinness at birth and glucose tolerance in seven-year-old children. Diabet Med. 1995;12:24-29.

128. Donker GA, Labarathe DR, Harrist RB, Selwyn BJ, Srinivasan SR, Wattigney W, Berenson GS. Low birth weight and serum lipid concentrations at age $7-11$ years in a biracial sample. $\mathrm{Am} J$ Epidemiol. 1997;145:398-407.

129. Malina RM, Katzmarzyk PT, Beunen G. Birth weight and its relationship to size attained and relative fat distribution at 7 to 12 years of age. Obes Res. 1996;4:385-390.

130. Okosun IS, Liao Y, Rotimi CN, Dever GEA, Cooper RS. Impact of birth weight on ethnic variations in subcutaneous and central adiposity in American children aged 5-11 years. A study from the third National Health and Nutrition Examination Survey. Int J Obes. 2000;24:479-484.

131. Hediger ML, Overpeck MD, Kuczmarski RJ, McGlynn A, Maurer KR, Davis WW. Muscularity and fatness of infants and young children born small-for-gestational-age. Pediatrics. 1998;102:e60.

132. Hediger ML, Overpeck MD, McGlynn A, Kuczmarski RJ, Maurer KR, Davis WW. Growth and fatness at three to six years of age of children born small- or large-for-gestational age. Pediatrics. 1999; 104:e33.

133. Hulman S, Kushner H, Katz S, Falkner B. Can cardiovascular risk be predicted by newborn, childhood, and adolescent body size? An examination of longitudinal data in urban African Americans. J Pediatr. 1998;132:90-97.

134. Martorell R, Ramakrishnan U, Schroeder DG, Melgar P, Neufeld L. Intrauterine growth retardation, body size, body composition and physical performance in adolescence. Eur $J$ Clin Nutr. 1998;52:S1-S53.

135. Forsén T, Nissinen A, Toumiletho J, Notkola IL, Eriksson J, Vinni S. Growth in childhood and blood pressure in Finnish children. J Hum Hypertens. 1998;12:397-402.

136. Donker GA, Labarthe DR, Harrist RB, Selwyn BJ, Wattigney W, Berenson GS. Low birth weight and blood pressure at age 7-11 years in a biracial sample. Am J Epidemiol. 1997;145:387. 397.

137. Ong KKL, Ahmed ML, Emmett PM, Preece MA, Dunger DB. Association between postnatal catch-up growth and obesity in childhood: prospective cohort study. BMJ. 2000;320:967-971.

138. Freedman DS, Serdula MK, Srinivasan SR, Berenson GS. Relation of circumferences and skinfold thicknesses to lipid and insulin concentrations in children and adolescents: the Bogalusa Heart Study. Am J Clin Nutr. 1999;69:308-317.

139. Eriksson JG, Forsén T, Tuomilehto J, Winter PD, Osmond C, Barker DJP. Catch-up growth in childhood and death from coronary heart disease: longitudinal study. BMJ. 1999;318:427-431.

140. Cuno SPM, Uiterwaal SA, Anthony S, Launer LJ, Witteman JCM, Trouwborst AMW, Hofman A, Grobbee DE. Birth weight, growth, and blood pressure. An annual follow-up study of children aged 5 trough 21 years. Hypertension. 1997;30:267-271.

141. Widdowson EM, McCane RA. A review: new thoughts on growth. Pediatr Res. 1975;9:154-156.

142. Winick M, Noble A. Cellular response in rats during malnutrition at various stages. $J$ Nutr. 1966;89:300-306.

143. Winick M, Fish I, Rosso P. Cellular recovery in rat tissues after a brief period of neonatal malnutrition. $J$ Nutr. 1968;95:623-626.

144. Langley SC, Jackson AA. Increased systolic blood pressure in adult rats induced by fetal exposure to maternal low protein diets. Clin Sci. 1994;86:217-222.

145. Dessau M, Crowther N, Lucas A, Hales CN. Programming of hepatic metabolism by low protein diet during early life. Diabetic med. 1994;11:91. 
146. Mackenzie HS, Brenner BM. Fewer nephrons at birth: a missing link in the etiology of essential hypertension. Am J Kidney Dis. 1995;26:91-98.

147. Aerts L, Pijnenborg R, Verhaege J, Holemans K, Van Assche FA. Fetal growth and development. In: Dornhorst A, Hadden DR, eds. Diabetes and pregnancy: an international approach to diagnosis and management. New York: John Wiley and Sons; 1996:77-97.

148. Phillips DIW. The 'fetal origins' hypothesis: role of programming of adrenocortical and sympathoadrenal function. In: O'Brien PMS, Wheeler T, Barker DJP, eds. Fetal programming: influence on development and disease in later life. London: RCOG Press; 1999:414-423.

149. Benediktsson R, Lindsay RS, Noble J, Seckl JR, Edwards CRW. Glucocorticoid exposure in utero: new model for adult hypertension. Lancet. 1993;341:339-341.

150. Edwards CRW, Benediktsson R, Lindsey RS, Seckl JR. Dysfunction of placental glucocorticoid barrier: link between fetal environment and adult hypertension? Lancet. 1993;341:355-357.

151. Waterland RA, Garza C. Potential mechanisms of metabolic imprinting that lead to chronic disease. Am J Clin Nutr. 1999;69:179-197.

152. Kelsey G, Constância M, Dean WL, Feil RP, Reik W. Genomic imprinting and fetal growth. In: O'Brien PMS, Wheeler T, Barker DJP, eds. Fetal programming: influences on development and disease in later life. London: RCOG Press; 1999:73-84.

153. Hattersley AT, Breards F, Ballantyne E, Appleton M, Harvey R, Ellard S. Mutations in the glucokinase gene of the fetus result in reduced birth weight. Nature Genet. 1998;19:268-269.

154. Dunger DB, Ong KKL, Huxtable SJ, Sherriff A, Woods KA, Ahmed ML, Golding J, Pembrey ME, Ring S, Bennet ST, Todd JA. Association of the INS VNTR with size at birth. Nature Genet. 1998;19:98-100.

155. Casteels K, Ong K, Phillips D, Bendall H, Pembrey M, Poulton J, Dunger D. Mitochondrial 16189 variant, thinness at birth, and type-2 diabetes. Lancet. 1999;353:1499-1500.

156. Barker DJP. Fetal programming and public health. In: O'Brien PMS, Wheeler T, Barker DJP, eds. Fetal programming: influences on development and disease in later life. London: RCOG Press; 1999.

157. Harding JE. The nutritional basis of the fetal origins of adult disease. Int $J$ Epidemiol. 2001;30:15-23.

158. Dahri S, Snoeck A, Reusens-Billen B, Remacle C, Hoet JJ. Islet function in offspring of mothers on low-protein diet during gestation. Diabetes. 1991;40:115-120.

159. Langley SC, Browne RF, Jackson AA. Altered glucose tolerance in rats exposed to maternal low protein diets in utero. Comp Biochem Physiol. 1994;109:223-229.

160. Desai M, Crowther NJ, Ozanne SE, Lucas A, Hales CN. Adult glucose and lipid metabolism may be programmed during fetal life. Biochem Soc Trans. 1995;23:331-335.

161. Woodall SM, Johnston BM, Breier BH, Gluckman PD. Chronic maternal undernutrition in the rat leads to delayed postnatal growth and elevated blood pressure of offspring. Pediatr Res. 1996; $40: 438-443$.

162. Hanson MA, Spencer JAD, Rodeck $\mathrm{CH}$, eds. Fetus and neonate. Physiology and clinical applications; Volume 3: Growth. Cambridge: Cambridge University Press; 1995.

163. Godfrey KM, Robinson S, Barker DJP, Osmond C. Maternal nutrition in early and late pregnancy in relation to placental and fetal growth. BMJ. 1996;312:410-414.

164. Godfrey KM, Barker DJP, Robinson S, Osmond C. Maternal birthweight and diet in pregnancy in relation to the infant's thinness at birth. Br J Obstet Gynaecol. 1997;104:663-667.

165. Campbell DM, Hall MH, Barker DJP, Cross J, Shiell AW, Godfrey KM. Diet in pregnancy and the offspring's blood pressure 40 years later. Br J Obstet Gynaecol. 1996;103:273-280.

166. Leon DA. Commentary: Getting to grips with the fetal programming - aspects of a rapidly evolving agenda. Int J Epidemiol. 2001;30:96-98.

167. Cherif H, Reusens B, Ahn MT, Hoet JJ, Remacle C. Effects of taurine on the insulin secretion of rat fetal islets from dams fed a low-protein diet. J Endocrinol. 1998;159:341-348. 
168. Olson SF. Further on the association between retarded foetal growth and adult cardiovascular disease. Could low intake of marine diets be a common cause? J Clin Epidemiol. 1994;47:565569.

169. James WPT. Long-term fetal programming of body composition and longevity. Nutr Rev. 1997;55:S31-S43.

170. Sattar N, Berry C, Greer IA. Essential fatty acids in relation to pregnancy complications and fetal development. Br J Obstet Gynaecol. 1998;105:1248-1255.

171. Keys A, Parlin RW. Serum cholesterol response to changes in dietary lipids. Am J Clin Nutr. 1966;19:175-181.

172. GISSI-Preventione Investigators (Groupo Italiano per lo Studio della Sopravvivenza nell'Infarto miocardico). Dietary supplementation with $\mathrm{n}-3$ polyunsaturated fatty acids and vitamin $\mathrm{E}$ after myocardial infaretion: results of the GISSI-Preventione trail. Lancet. 1999;354:447-455.

173. Knapp HR. Fatty acids and hypertension. World Rev Nutr Dev. 1994;76:9-14.

174. Mensink RP, Katan MB. Effect of dietary fatty acids on serum lipids and lipoproteins. A metaanalysis of 27 trials. Arterioscler Thromb. 1992;12:911-919.

175. Storlien LH, Baur LA, Kriketos AD, Pan DA, Coony GJ, Jenkins AB, Calvet GD, Campbell LV. Dietary fats and insulin action. Diabetalogia. 1996;39:621-631.

176. Storlien LH, Kritekos AD, Calvert GD, Baur LA, Jenkins AB. Fatty acids, triglycerides and syndromes of insulin resistance. Prostagaldins Leukotr Essent Fatty Acids. 1997:57:379-385.

177. Vessby B. Dietary fat and insulin action in humans. Br J Nutr. 2000;83:S91-S96.

178. Horrobin DF. Fatty acid metabolism in health and disease: the role of -6-desaturase. Am J Clin Nutr. 1993;57:732S-737S.

179. Nordøy A. Dietary fatty acids and coronary heart disease. Lipids. 1999;34:S19-S22.

180. Miller GJ. Dietary fatty acids and blood coagulation. Prostaglandins Leucotr Essent Fatty Acids. 1997;57:389-394.

181. Kris-Etherton P, Daniels SR, Eckel RH, Engler M, Howard BV, Krauss RM, Lichtenstein AH, Sacks F, Jeor SS, Stampfer M. Summary of the scientific conference on dietary fatty acids and cardiovascular health. Conference summary from the nutrition committee of the American Heart Association. Circulation. 2001;103:1034-1039.

182. Hu FB, Stampfer MJ, Manson JE, Rimm E, Colditz GA, Rosner BA, Hennekens CH, Willett WC. Dietary fat intake and the risk of coronary heart disease in women. $N$ Eng $J$ Med. 1997;337:1491-1499.

183. Siscovick DS, Raghunathan TE, King I, Weinmann S, Bovbjerg VE, Kushi L, Cobb LA, Copass MK, Psaty BM, Lemaitre R, Retzlaff B, Knopp RH. Dietary intake of long-chain n-3 polyunsaturated fatty acids and the risk of primary cardiac arrest. Am J Clin Nutr. 2000;71:208S$212 \mathrm{~S}$.

184. Weisinger HS, Armitage JA, Sinclear AJ, Vingrys AJ, Burns PL, Weisinger RS. Perinatal omega-3 fatty acid deficiency affects blood pressure later in life. Nature Med. 2001;7:258-259.

185. Burr GO, Burr MM. A new deficiency disease produced by rigid exclusion of fat from the diet. $J$ Biol Chem. 1929:82:345-367.

186. Burr GO, Burr MM. On the nature and role of the fatty acids essential in nutrition. $J$ Biol Chem. 1930;86:587-621.

187. Hansen AE, Wiese HF, Boelsche AN, Haggard ME, Adam DJD, Davis H. Role of linoleic acid in infant nutrition. Pediatrics. 1963:31:171-192.

188. Holman RT. Essential fatty acid deficiency in humans. Dietary lipids and postnatal development. New York: Raven; 1973

189. Wene JD, Connor WE, Denbesten L. The development of essential fatty acid deficiency in healthy men fed fat-free diets intravenously and orally. J Clin Invest. 1975;56:127-134.

190. Caldwell MD, Jonsson HT, Othersen HB. Essential fatty acid deficiency in an infant receiving prolonged parental alimentation. J Pediatr. 1972;81:894-898. 
191. Bjerve KS. N-3 fatty acid deficiency in man. J Intern Med. 1989;225:171-175.

192. Hansen HS, Jensen B. Essential function of linoleic acid esterified in acylglucosylceramide and acylceramide in maintaining the epidermal water barrier. Evidence from feeding studies with oleate, lineolate, arachidonate, culombinate and $\alpha$-linolenate. Biochim Biophys Acta. 1985;834:357-363.

193. Hansen HS. The essential nature of linoleic acid in mammals. TIBS. 1986;11:263-265.

194. Bergström S, Danielsson H, Samuelsson B. The enzymatic formation of prostaglandin $E_{2}$ from arachidonic acid. Biochim Biophys Acta. 1964;90:207-210.

195. Dorp DAv, Beerthuis RK, Nugteren DH, Vonkeman K. The biosynthesis of prostaglandins. Biochim Biophys Acta. 1964;90:204-207.

196. Sellmayer A, Koletzko B. Long-chain polyunsaturated fatty acids and eicosanoids in infants physiological and pathophysiological aspects and open questions. Lipids. 1999;34:199-205.

197. James MJ, Gibson RA, Cleland LG. Dietary polyunsaturated fatty acids and inflammatory mediator production. Am J Clin Nutr. 2000;71:343S-348S.

198. Ginsberg BH, Brown TJ, Simon I, Spector AA. Effect of the membrane lipid environment on the properties of insulin receptors. Diabetes. 1981;30:773-780.

199. Holman RT, Johnson SB, Ogburn PL. Deficiency of essential fatty acids and membrane fluidity during pregnancy and lactation. Proc Natl Acad Sci USA. 1991;88:4835-4839.

200. Innes SM. Essential fatty acids in growth and development. Prog Lipid Res. 1991;30:39-103.

201. Kurlak LO, Stephenson TJ. Plausable explainantions for effects of long chain polyunsaturated fatty acids (LCPUFA) on neonates. Arch Dis Child Fetal Neonatal Ed. 1999;80:F148-F154.

202. Clarke SD, Jump DB. Polyunsaturated fatty acid regulation of hepatic gene transcription. Lipids. 1996;31:S7-S11.

203. Clarke SD, Thuillier P, Baillie RA, Sha X. Peroxisome proliferator-activated receptors: a family of lipid-activated transcription factors. Am J Clin Nutr. 1999;70:566-571.

204. Raclot T, Groscolas R, Langin B, Ferre T. Site-specific regulation of gene expression by n-3 polyunsaturated fatty acids in rat white adipose tissues. J lipid Res. 1997;38:1963-1972.

205. Holman RT. The ratio of trienoic : tetraenoic acids in tissue lipids as a measure of essential fatty acid requirement. $J$ Nutr. 1960;70:405-409.

206. Hoffman DR, Uauy R. Essentiality of dietary omega-3 fatty acids for premature infants; plasma and red blood cell fatty acid composition. Lipids. 1992;27:886-895.

207. Hornstra G, Houwelingen ACv, Simonis M, Gerrard JM. Fatty acid composition of umbilical arteries and veins: possible implications for fetal EFA-status. Lipids. 1989;24:511-517.

208. Crawford MA, Costeloe K, Doyle W, Leighfield MJ, Lennon EA, Meadows N. Potential diagnostic value of the umbilical artery as a definition of neural fatty acid status of the fetus during growth: the umbilical artery as a diagnostic tool. Biochem Soc Trans. 1990;18:761-766.

209. Hornstra G, Al MDM, Houwelingen ACv, Foreman-van Drongelen MMHP. Essential fatty acids in pregnancy and early human development. Eur J Obstet Gynecol Reprod Biol. 1995;61:57-62.

210. Salem N, Wegher B, Mena P, Uauy R. Arachidonic and docosahexaenoic acids are biosynthesized from their 18-carbon precursors in human infants. Proc Natl Acad Sci USA. 1996;93:49-54.

211. Koletzko B, Decsi T, Demmelmair H. Arachidonic acid supply and metabolism in human infants born at full term. Lipids. 1996;31:79-83.

212. Rodriguez A, Sarda P, Nessmann C, Boulot P, Poisson JP, Leger CL, Descomps B. Fatty acid desaturase activities and polyunsaturated fatty acid composition in human liver between the seventeeth and thirty-sixth gestational weeks. Am J Obstet Gynecol. 1998;179:1063-1070.

213. Hamosh M, Salem N. Long-chain polyunsaturated fatty acids. Biol Neonate. 1998;74:106-120.

214. Al MD, Hornstra G, Schouw YTvd, Bulstra-Ramakers MT, Huisjes HJ. Biochemical EFA status of mothers and their neonates after normal pregnancy. Early Hum Dev, 1990;24:239-48. 
215. Al MD, Badart-Smook A, Houwelingen ACv, Hasaart TH, Hornstra G. Fat intake of women during normal pregnancy: relationship with maternal and neonatal essential fatty acid status. $J$ Am Coll Nutr. 1996;15:49-55.

216. Al MDM, Houwelingen ACv, Hornstra G. Long-chain polyunsaturated fatty acids, pregnancy, and pregnancy outcome. Am J Clin Nutr. 2000;71:285S-291S.

217. Crawford MA. Placental delivery of arachidonic and docosahexaenoic acids: implications for the lipid nutrition of preterm infants. Am J Clin Nutr. 2000;71:275S-284S.

218. Dutta-Roy AK. Transport mechanisms for long-chain polyunsaturated fatty acids in the human placenta. Am J Clin Nutr. 2000;71:315S-322S.

219. Haggarty P, Ashton J, Joynson M, Abramovich DR, Page K. Effect of maternal polyunsaturated fatty acid concentration on transport by the human placenta. Biol Neonate. 1999;75:350-359.

220. Hansen AE, Wiese HF, Adam DJD, al. e. Influence of diet on blood serum lipids in pregnant women and newborn infants. Am J Clin Nutr. 1964;15:11-19.

221. Friedman Z, Danon A, Lamberth EL, Mann WJ. Cord blood fatty acid composition in infants and in their mothers during the third trimester. J Pediatr. 1978;92:461-466.

222. Crawford MA, Hassam AG, Williams G. Essential fatty acids and fetal brain growth. Lancet. $1976 ;$;:452-453.

223. Hoving ED, Beusekom CMv, Nijboer HJ, Muskiet FAJ. Gestational age dependency of essential fatty acids in cord plasma cholesterol esters and triglycerides. Pediatr Res. 1994;35:461-469.

224. Houwelingen ACv, Puls J, Hornstra G. Essential fatty acid status during early human development. Early Hum Dev. 1992;31:97-111.

225. Houwelingen ACv, Foreman-van Drongelen MMHP, Nicolini U, Nicolaides KH, Al MDM, Kester ADM, Hornstra G. Essential fatty acid status of fetal plasma phospholipids: similar to postnatal values obtained at comparable gestational ages. Early Hum Dev. 1996;46:141-152.

226. Innes SM. Maternal diet, length of gestation, and long-chain polyunsaturated fatty acid status of infants at birth. Am J Clin Nutr. 1999;70:181-182.

227. Foreman-van Drongelen $\mathrm{MMHP}, \mathrm{Al} \mathrm{MDM}$, Houwelingen $\mathrm{ACv}, \mathrm{Blanco} \mathrm{CE}$, Hornstra $\mathrm{G}$. Comparison between the essential fatty acid status of preterm and full-term infants, measured in umbilical vessel walls. Early Hum Dev. 1995;42:241-251.

228. Crawford MA, Doyle W, Drury P, Lennon A, Costeloe K, Leighfield M. n-3 and n-6 fatty acids during early human development. J Intern Med. 1989;225:159-169.

229. Koletzko B, Braun M. Arachidonic acid and early human growth: is there a relation? Ann Nutr Metab. 1991;35:128-131.

230. Leaf AA, Leighfield MJ, Costeloe KL, Crawford MA. Long chain polyunsaturated fatty acids and fetal growth. Early Hum Dev. 1992;30:183-191.

231. Felton CV, Chang TC, Crook D, Marsh M, Robson SC, Spencer JAD. Umbilical vessel wall fatty acids after normal and retarded fetal growth. Arch Dis Child. 1994;70:F36-F39.

232. Foreman-van Drongelen MMHP, Houwelingen ACv, Kester ADM, Hasaart THM, Blanco CE, Hornstra G. Long-chain polyunsaturated fatty acids in pre-term infants: status at birth and its influence on postnatal levels. J Pediatr. 1995;126:611-618.

233. Woltil HA, Beusekom CMv, Schaafsma A, Muskiet FAJ, Okken A. Long-chain polyunsaturated fatty acid status and early growth of low birth weight infants. Eur J Pediatr. 1998;157:146-152.

234. Robillard PY, Christon R. Lipid intake during pregnancy in developing countries: possible effect of essential fatty acid deficiency on fetal growth. Prostaglandins Leukotr Essent Fatty Acids. 1992;48:139-142.

235. Olson SF, Hansen HS, Sorenson TIA, al. e. Intake of marine fat, rich in n-3 polyunsaturated fatty acids, may increase birth weight by prolonging gestation. Lancet. 1986;ii:367-369.

236. Olson SF, Sørensen JD, Secher NJ, Hedegaard M, Hendriksen TB, Hansen HS, Grant A. Randomised controlled trial of effect of fish-oil supplementation on pregnancy duration. Lancet. 1992;339:1003-1007. 
237. Olson SF, Secher NJ, Tabor A, Weber T, Walker JJ, Gluud C. Randomised clinical trials of fish oil supplementation in high risk pregnancies. Br J Obstet Gynaecol. 2000;107:382-395.

238. Bergner L, Susser MW. Low birth weight and prenatal nutrition: an interpretive review. Pediatrics. 1970;46:946.

239. Susser M. Maternal weight gain, infant birth weight, and diet: causal consequences. Am J Clin Nutr. 1990;53:1384.

240. Onis Md, Villar J, Gülmezoglu M. Nutritional intervention to prevent intrauterine growth retardation: evidence from randomized controlled trails. Eur J Clin Nutr. 1998;52:S83-S93.

241. Mathews F, Yudkin P, Neil A. Influence of maternal nutrition on outcome of pregnancy: prospective cohort study. BMJ. 1999;319:339-343.

242. Marenberg ME, Risch N, Berkman LF, Floderus B, Faire Ud. Genetic susceptibility to death from coronary heart disease in a study of twins. N Eng J Med. 1994;330:1041-1046.

243. Colditz GA, Rimm EB, Giovannucci E, Stampfer MJ, Rosner B, Willett WC. A prospective study of parental history of myocardial infarction and coronary artery disease in men. $\mathrm{Am} \mathrm{J}$ Cardiol. 1991;67:933-938.

244. Humphries SE. DNA polymorphisms of the apolipoprotein genes - their use in the investigation of the genetic component of hyperlipidaemia and atherosclerosis. Atherosclerosis. 1988;72:89108.

245. Ferrell RE. Genetics of the apolopoproteins and the contribution of allelic variation to quantitative variation in lipid and lipoprotein levels in the population. Curr Opin Lipidol. 1992;3:122-127.

246. Sing CF, Davignon J. Role of the apolipoprotein E polymorphism in determining normal plasma lipid and lipoprotein variation. Am J Hum Genet. 1985;37:268-285.

247. Boerwinkle E, Visvikis S, Welsh D, Steinmetz J, Hanash SM, Sing CF. The use of measured genotype information in the analysis of quantitative phenotypes in man. II. The role of the apolipoprotein E polymorphism in determining levels, variability and covariability of cholesterol, betalipoprotein and triglycerides in a sample of unrelated individuals. Am J Hum Genet. 1987; 27:567-582.

248. Eichner JE, Kuller LH, Ferrell RE, Meilahn EN, Kamboh MI. Phenotypic effects of apolipoprotein structural variation on lipid profiles. III. Contribution of apolipoprotein E phenotype to prediction of total cholesterol, apolipoprotein B, and low-density lipoprotein cholesterol in The Healthy Women Study. Arteriosclerosis. 1990;10:379-385.

249. Howard BV, Gidding SS, Liu K. Association of apolipoprotein E phenotype with plasma lipoproteins in African-American and white young adults. The CARDIA Study. Am J Epidemiol. 1998:148:859-868.

250. Smit M, Knijff Pd, Rosseneu M, Bury J, Klasen E, Frants R, Havekes LM. Apolipoprotein E polymorphism in the Netherlands and its effects on plasma lipid and apolipoprotein levels. Hum Genet. 1988;80:287-292.

251. Sanghera DK, Ferrell RE, Aston CE, McAllister AE, Kamboh MI, Kimm SYS. Quantitative effects of the apolipoprotein E polymorphism in a biracial sample of 9-10-year-old girls. Atherosclerosis. 1996;126:35-42.

252. Lapinleimu H, Viikari J, Niinikoski H, Tuominen J, Rönnemaa T, Välimäki I, Marniemi J, Jokinen E, Ehnholm C, Simell O. Impact of gender, apolipoprotein E phenotypes, and diet on serum lipids and lipoproteins in infancy. J Pediatr. 1997;131:825-832.

253. Srinivasan SR, Ehnholm C, Wattigney WA, Bao W, Berenson GS. The relation of apolipoprotein E polymorphism to multiple cardiovascular risk in children: the Bogalusa Heart Study. Atherosclerosis. 1996;123:33-42.

254. Srinivasan SR, Ehnholm C, Wattigney WA, Berenson GS. Influence of apolipoprotein E polymorphism on the tracking of childhood levels of serum lipids and lipoproteins over a 6-year period. The Bogalusa Heart Study. Atherosclerosis. 1996;127:73-79. 
255. Kallio MJT, Salmenperă L, Siimes A, Perheentupa J, Gylling H, Miettinen TA. The apolipoprotein E phenotype has strong influence on tracking of serum cholesterol and lipoprotein levels in children: a follow-up study from birth to the age of 11 years. Pediatr Res. 1998;43:381385.

256. Srinivasan SR, Ehnholm C, Elkasabany A, Berenson G. Influence of apolipoprotein E polymorphism on serum lipids and lipoprotein changes from childhood to adulthood. The Bogalusa Heart Study. Atherosclerosis. 1999;143:435-443.

257. Fulton JE, Dai S, Grunbaum JA, Boerwinkle E, Labarthe DR. Apolipoprotein E affects serial changes in total and low-density lipoprotein cholesterol in adolescent girls: Project HeartBeat! Metabolism. 1999;48:285-290.

258. Tiret L, Knijff Pd, Menzel HJ, Ehnholm C, Nicaud V, Havekes LM. Apo E polymorfism and predisposition to coronary heart disease in youths of different european populations. The EARS Study. Arterioscler Thromb. 1994;14:1617-1624.

259. Porka KVK, Taimela S, Kontula K, Lehtimäki T, Aalto-Setallă K, Ákerblom HK, Viikari JSA. Variability gene effects of DNA polymorphisms at the apo B, apo ALCIII and apo E loci on serum lipids: the Cardiovascular Risk in Young Finns Study. Clin Genet. 1994;45:113-121.

260. Martin LJ, Connolly PW, Nancoo D, Wood N, Zhang ZJ, Maguire G, Quinet E, Tall AR, Marcel $\mathrm{YL}, \mathrm{McPherson} \mathrm{R}$. Cholesteryl ester transfer protein and high density lipoprotein responses to cholesterol feeding in men: relationship to apolipoprotein E genotype. J Lipid Res. 1993;34:437446.

261. Moulin P. Cholesteryl ester transfer protein: an enigmatic protein. Horm Res 1996;45:238-244.

262. Kondo I, Berg K, Drayna D, Lawn R. DNA polymorphism at the locus for human cholesteryl ester transfer protein (CETP) is associated with high density lipoprotein cholesterol and apolipoprotein levels. Clin Genet. 1989;35:49-56.

263. Kuivenhoven JA, Knijf Pd, Boer JMA, Smalheer HA, Botma GJ, Seidell JC, Kastelein JJP, Pritchard PH. Heterogeneity at the CETP gene locus. Influence on plasma CETP concentrations and HDL cholesterol levels. Aterioscler Thromb Vasc Biol. 1997; 17:560-568.

264. Gudnason V, Kakko S, Nicaud V, Savolainen MJ, Kesăniemi YA, Tahvanainen E, Humphries S. Cholesteryl ester transfer protein gene effect on CETP activity and plasma high-density lipoprotein in European populations. Eur J Clin Invest. 1999;29:116-128.

265. Kuivenhoven JA, Jukema JW, Zwinderman AH, Knijff Pd, McPherson R, Bruschke AVG, Lie $\mathrm{KI}$, Kastelein JJP. The role of a common variant of the cholesteryl ester transfer protein gene in the progression of coronary atherosclerosis. N Eng J Med. 1998;338:86-93.

266. Clifton PM, Abbey M. Genetic control of response to dietary fat and cholesterol. World Rev Nutr Diet. 1997;80:1-14.

267. Ordovas JM, Lopez-Miranda J, Mata P, Perez-Jimenez F. Gene-environment interactions in lipoprotein metabolism. Nutr Metab Cardiovasc Dis. 1998;8:47-61.

268. Boer JMA, Ehnholm C, Menzel HJ, Havekes LM, Rosseneu M, O'Reilly DSJ, Tiret L. Interactions between lifestyle-related factors and the apoE polymorphism on plasma lipids and lipoproteins. The EARS Study. Arterioscler Thromb Vasc Biol. 1997;17:1675-1681.

269. Berg K, Kondo I, Drayna D, Lawn R. "Variability gene" effect of cholesteryl ester transfer protein (CETP) genes. Clin Genet. 1989;35:437-445.

270. Minihane AM, Khan S, Leigh-Firbank EC, Talmud P, Wright JW, Murphy MC, Griffin BA, Williams CM. ApoE polymorphism and fish oil supplementation in subjects with an atherogenic lipoprotein phenotype. Arterioscler Thromb Vasc Biol. 2000;20:1990-1997.

271. Ye SQ, Kwiterovich PO. Influence of genetic polymorphisms on responsiveness to dietary fat and cholesterol. Am J Clin Nutr. 2000;72:1275S-1284S.

272. Erkkilă AT, Sarkkinen ES, Lindi V, Lehto S, Laakso M, Uusitupa M. APOE polymorphism and the hypertriglyceridemic effect of dietary sucrose. Am J Clin Nutr. 2001;73:746-752. 
273. Henry JA, Bolla M, Osmond C, Fall C, Barker DJP, Humphries SE. The effects of genotype ani infant weight on adult plasma levels of fibrinogen, factor VII, and LDL cholesterol are additive J Med Genet. 1997;34:553-558.

274. Al MD, Houwelingen ACv, Kester AD, Hasaart TH, de Jong AE, Hornstra G. Maternal essentia fatty acid patterns during normal pregnancy and their relationship to the neonatal essential fatt acid status. Br J Nutr. 1995; 74:55-68.

275. Al MD, Houwelingen ACv, Badart-Smook A, Hasaart TH, Roumen FJ, Hornstra G. Th essential fatty acid status of mother and child in pregnancy-induced hypertension: a prospectiv longitudinal study. Am J Obstet Gynecol. 1995;172:1605-14.

276. Badart-Smook A, Houwelingen ACv, Al MDM, Kester ADM, Hornstra G. Fetal growth i associated positively with maternal intake of riboflavin and negatively with maternal intake o linoleic acid. J Am Diet Assoc. 1997;97:867-870.

277. Kloosterman GJ. On intrauterine growth: The significance of prenatal care. Int J Gyneace Obstet. 1970;8:895-912.

278. World Health Organization. Physical status: the use and interpretation of anthropometry. Repoi of a WHO expert committee. World Health Organ Tech Rep Ser. 1995;854. 
Chapter 2 The n-3 and n-6 polyunsaturated fatty acid composition of plasma phospholipids in pregnant women and their infants. Relationship with maternal linoleic acid intake

P. Rump and G. Hornstra

Clin Chem Lab Med 2002;40:32-39 


\begin{abstract}
The availability of long-chain polyunsaturated fatty acids during infancy has bee related to neonatal growth and development. Fatty acid concentration at birth is a important predictor of postnatal level. The primary aim of this study was to provide ; description of the distribution of $n-3$ and $n-6$ polyunsaturated fatty acids in the plasmi phospholipid fraction of pregnant women remaining on a Western-style diet and theineonates. The plasma phospholipid polyunsaturated fatty acid composition wa; determined by gas-liquid chromatography in 889 mother-infant pairs. Blood sample; were taken during the first, second and third trimester of pregnancy, at delivery, and from the umbilical vein at birth. Mean $( \pm S D)$ fatty acid concentrations are reported it $\mathrm{mg} / \mathrm{L}$ and as percentage of total fatty acids (\% wt/wt). In addition, the $10^{\text {th }}, 25^{\text {th }}, 50^{\text {th }}$, $75^{\text {th }}$ and $90^{\text {th }}$ percentiles are given. The distribution of docosahexaenoic acid $(22: 6 \mathrm{n}-3)$ and arachidonic acid (20:4n-6) concentrations in umbilical plasma phospholipids $\mathrm{i}$; also reported as a function of gestational age and maternal linoleic acid intake duriny pregnancy. This data can be used as a reference for future studies and may aid is identifying term infants with a relatively low long-chain polyene status at birth.
\end{abstract}




\section{Introduction}

The biological significance of $n-3$ and $n-6$ polyunsaturated fatty acids (PUFAs) for optimal neonatal growth and development has received considerable attention. ${ }^{1-4}$ The involved long-chain polyenes (LCPs), such as arachidonic acid (20:4n-6) and docosahexaenoic acid $(22: 6 n-3)$, can be derived from the parent essential fatty acids linoleic acid (18:2n-6) and $\alpha$-linolenic acid (18:3n-3), respectively (see Figure 1.2, Chapter 1$).{ }^{1}$ The derivatives dihomo- $\gamma$-linolenic acid $(20: 3 n-6)$, arachidonic acid and eicosapentaenoic acid (20:5n-3) are direct precursors of prostaglandins and other eicosanoids. Arachidonic acid and docosahexaenoic acid are important structural and functional components of cellular membranes. Concentrations of these PUFAs in plasma lipid fractions are often used as a marker for dietary exposure or to indicate a subject's essential fatty acid status. The availability of detailed reference values for plasma PUFA concentrations in pregnant women and their neonates might be useful for comparative investigations and for metabolic and nutritional studies.

Although fatty acid concentrations in maternal and umbilical plasma lipid fractions have been described before, ${ }^{5-11}$ most of the available information is based on relatively small numbers of subjects. To our knowledge, none of the previously published papers reported percentile values for absolute $(\mathrm{mg} / \mathrm{L})$ and relative $(\% \mathrm{wt} / \mathrm{wt})$ PUFA concentrations. During a longitudinal observational study, we determined the fatty acid composition of plasma phospholipids in a large population of pregnant women and their neonates. The main purpose of the present paper was to describe the distribution of the $n-3$ and $n-6$ PUFA concentrations. In addition, we studied the relationship between the fatty acid profiles and the maternal linoleic acid intake in a sub-sample of the population.

\section{Subjects and Methods}

\section{Study population}

At their first visit to the antenatal clinic, pregnant women were asked to participate in a longitudinal observational study of the changes in essential fatty acid status during pregnancy and their relation to the outcome of pregnancy. ${ }^{6,12,13}$ Three antenatal clinics participated: the University Hospital in Maastricht, Hospital "De Wever" in Heerlen and the School for Midwifery in Kerkrade. These centers are all located in the province of Limburg in the southern part of the Netherlands. The entry criteria for the study were gestational age of less than 16 weeks at and no signs of cardiovascular, neurological, renal, or metabolic disorders. Plasma samples were available in a total of 1212 motherinfant pairs. After excluding cases on the basis of multiple pregnancy $(n=24)$, premature delivery (pregnancy duration $<37$ completed weeks, $n=89$ ), post-term delivery (pregnancy duration $\geq 43$ completed weeks, $n=4)$, infant death $(n=5)$, inappropriate birth weight ( $\leq 2.3$ th percentile or $\geq 97.7$ percentile, according to Dutch 
reference data, $\left.{ }^{14} n=36\right)$, unknown gestational age or birth weight $(n=37)$, maternal diabetes $(n=40)$ or maternal hypertension $(n=162)$, a total study population of 889 women and neonates was left for analysis. Approval for this study was obtained from the Ethics Committee of the University Hospital Maastricht and all participating women gave their written informed consent.

Local hospital staff members recorded individual maternal and infant characteristics on a standardized data sheet. Additional information was obtained by questionnaires or retrieved from medical records. Pregnancy duration (in weeks) was calculated from the recorded date of delivery and the self-reported first day of the last menstrual period, and fractions were expressed in decimals. If the last menstrual period was unknown, gestational age was based on the early ultrasound measurements. The maternal and infant characteristics are summarized in Table 2.1.

\section{Dietary intake of fatty acids}

To determine the habitual dietary fatty acid intake of the pregnant women, a crosscheck dietary history was carried out in a sub-sample of the study population during the second trimester, at approximately 22 weeks of gestation. A trained dietitian questioned 288 selected women about their usual food consumption patterns over the preceding 2 months. The food consumption data were encoded according to the system of the Netherlands Nutrition Databank and converted into nutrient intake data using the Netherlands food table (version NL3a). This version of the Netherlands food table does not allow the calculation of individual $n-3$ and $n-6$ fatty acid intake other than that of linoleic acid (18:2n-6) and total PUFA (for more details see $\mathrm{Al}$ et al. ${ }^{13}$ and BadartSmook et al. ${ }^{15}$ ).

\section{Blood collection and determination of fatty acid composition}

Maternal venous blood samples were collected in EDTA-treated tubes during the first, second, and third trimester, and after delivery. Directly after parturition, an additional blood sample was obtained from the umbilical vein. Plasma was separated by centrifugation and stored under nitrogen at $-80{ }^{\circ} \mathrm{C}$ until analysis. The fatty acid composition of maternal and umbilical plasma phospholipids was determined by capillary gas-liquid chromatography, as described before. ${ }^{6}$ Briefly, after the addition of 1, 2-dinonadecanoyl phosphatidyl-choline (internal standard) total lipid extracts from $100 \mu \mathrm{L}$ plasma were prepared using modified Folch extraction. ${ }^{16,17}$ Phospholipids were isolated by solid phase separation from total lipid extracts on aminopropyl-silica columns ( $\pm 90 \%$ recovery). ${ }^{18}$ To check for carry-over of other lipid fractions during this procedure, heptadecaenoic acid (17:1) was added to the samples. After saponification of the isolated phospholipids, fatty acids were converted to the corresponding methyl esters (FAMEs). ${ }^{19}$ The FAMEs were analyzed by capillary gas-liquid chromatography using a $50 \mathrm{~m}$ CP-Sil 5 CB non-polar capillary column (Chrompack, Middelburg, the Netherlands). 
Table 2.1: Characteristics of the study population $(n=889)$.

\begin{tabular}{|c|c|}
\hline & $\begin{array}{l}\text { Mean } \pm \mathrm{SD} \\
\text { or frequency }(\%)\end{array}$ \\
\hline \multicolumn{2}{|l|}{ Maternal characteristics } \\
\hline Age (y) & $29.3 \pm 4.4$ \\
\hline Height $(\mathrm{cm})$ & $166.3 \pm 6.4$ \\
\hline Weight at study entry (kg) & $65.7 \pm 11.3$ \\
\hline Weight increase during pregnancy $(\mathrm{kg})$ & $11.6 \pm 4.1$ \\
\hline \multicolumn{2}{|l|}{ Parity at entry $(\%)$} \\
\hline nullipara & 73.2 \\
\hline primipara & 21.7 \\
\hline multipara & 5.1 \\
\hline \multicolumn{2}{|l|}{ Dietary fatty acid intake $(\mathrm{g} / \text { day })^{1}$} \\
\hline total fat & $97.7 \pm 33.5$ \\
\hline PUFA & $18.4 \pm 8.3$ \\
\hline MUFA & $37.1 \pm 13.1$ \\
\hline SFA & $40.7 \pm 13.7$ \\
\hline linoleic acid $(18: 2 n-6)$ & $15.6 \pm 7.5$ \\
\hline \multicolumn{2}{|l|}{ Infant characteristics } \\
\hline Sex $(\%$ female $)$ & 45.2 \\
\hline \multicolumn{2}{|l|}{ Weight (g) } \\
\hline boys & $3397 \pm 420$ \\
\hline girls & $3277 \pm 420$ \\
\hline Weight $\leq 10^{\text {th }}$ percentile $(\%)^{2}$ & 11.5 \\
\hline Weight $\geq 90^{\text {th }}$ percentile $(\%)^{2}$ & 5.6 \\
\hline
\end{tabular}

PUFA, polyunsaturated fatty acids; MUFA, monounsaturated fatty acids; SFA, saturated fatty acids.

${ }^{1}$ Measured by dietary history midway gestation in a sub-sample of 288 women.

${ }^{2}$ According to Dutch reference data (appropriate for gestational age, infant sex and birth order). ${ }^{14}$ Note that infants with a birth weight $\leq 2.3^{\text {rd }}$ percentile or $\geq 97.7^{\text {th }}$ percentile were excluded.

Fatty acid concentrations are reported in $\mathrm{mg} / \mathrm{L}$ plasma and as a percentage of total fatty acids (\% wt $/ w t)$. Quantification was based on the amount of internal standard (19:0) recovered, assuming that equal mass amounts of long-chain polyunsaturated fatty acid methyl esters give rise to equal gas chromatography (GC) peak areas. ${ }^{20}$

\section{Statistical analysis}

Data are presented as mean \pm standard deviation (SD). In addition, the $10^{\text {th }}, 25^{\text {th }}, 50^{\text {th }}$ (median), $75^{\text {th }}$, and the $90^{\text {th }}$ percentiles are reported. Spearman rank correlation tests were applied to study the effect of the gestational age at birth and maternal PUFA intake. Differences between means were evaluated by either paired or unpaired twotailed Student's $t$ tests or by non-parametric Wilcoxon signed rank tests. Due to incomplete data, not all analyses were based on the same number of subjects. A twotailed $P$ value $<0.05$ was considered statistically significant. All statistical analyses were performed using the StatView version 4.5 statistical software (Abacus Concepts Inc., Berkeley, CA, USA). 


\section{Results}

The mean ( \pm SD) and percentiles of PUFA concentration in maternal plasma phospholipids at delivery are given in Table 2.2 (the data on the distribution of PUFA concentrations observed during pregnancy are given in Appendices $A, B$, and $C$ ). The women tended to maintain their ranking order with respect to their plasma phospholipid fatty acid concentrations during pregnancy (Spearman rank correlation coefficients for the associations between values at the first trimester of pregnancy and those determined at delivery were 0.52 for linoleic acid, 0.63 for arachidonic acid, and 0.56 for docosahexaenoic acid; $P<0.0001$ for all). The PUFA composition of umbilical plasma phospholipids is reported for boys and girls separately (Tables $2.3 \& 2.4$ ). The concentrations of the other identified fatty acids are reported in Table 2.5.

The well-known differences in PUFA concentrations between mothers and neonates were observed. The total concentration of phospholipid-associated fatty acids was $45.39 \mathrm{mg} / \mathrm{L}(95 \% \mathrm{CI}: 27.71$ - 63.07) higher in girls than in boys. A similar difference of approximately $7 \%$ was observed for the individual concentrations $(\mathrm{mg} / \mathrm{L})$ of the predominant fatty acids (Tables $2.3 \& 2.4$ ). However, the proportions ( $\% \mathrm{wt} / \mathrm{wt}$ ) of individual fatty acids did not differ significantly between boys and girls. These findings indicate that it was the size of the phospholipid pool rather than its composition that differed. The PUFA concentrations in maternal plasma were important predictors of the PUFA concentrations in neonatal plasma (Spearman $\mathrm{r}=0.39-0.50 ; P$ $<0.0001$ for all PUFAs with a mean concentration $>1 \% \mathrm{wt} / \mathrm{wt}$ ).

The PUFA composition of umbilical cord plasma phospholipids was related to the gestational age at birth (maternal PUFA concentrations at delivery were not related to the length of gestation). The concentration (\% wt/wt and $\mathrm{mg} / \mathrm{L})$ of $22: 6 \mathrm{n}-3$ was positively related to gestational age at birth $(P<0.0001$ for both). In contrast, concentrations of arachidonic acid (20:4n-6), dihomo- $\gamma$-linolenic acid (20:3n-6) and docosapentaenoic acid $(22: 5 n-6)$ hardly differed between infants born at different gestational ages. Moreover, concentrations ( $\mathrm{mg} / \mathrm{L}$ and $\% \mathrm{wt} / \mathrm{wt}$ ) of docosapentaenoic acid (22:5-3) and docoasatetraenoic acid (22:4n-6) were positively associated with the length of gestation, whereas concentrations of eicosatrienoic acid acid (20:3n-9) were negatively related to gestational age at birth $(P<0.01$ for all $)$.

Legend to Table 2.2 (next page): Mean \pm SD gestational age at delivery was $40.2 \pm 1.2 \mathrm{wk}$. ND, not detectable; $\sum \mathrm{n}-6$ and $\sum \mathrm{n}-3$, sum of all $\mathrm{n}-6$ and $\mathrm{n}-3$ fatty acids; $\sum \mathrm{n}-6$ LCP, sum of $\mathrm{n}-6$ long-chain polyenes $(20: 3 n-6,20: 4 n-6,22: 4 n-6$, and $22: 5 n-6) ; \sum n-3$ LCP, sum of $n-3$ long-chain polyenes $(20: 4 n-3,20: 5 n-3,22: 5 n-3$, and $22: 6 n-3): \sum n-7+n-9$, sum of all $n-7$ and $n-9$ fatty acids; $\Sigma$ SFA, ¿MUFA, ¿PUFA, sum of all saturated, monounsaturated, and polyunsaturated fatty acids, respectively. The total amount and all individual fatty acid concentrations ( $\mathrm{mg} / \mathrm{L}$ and $\% \mathrm{wt} / \mathrm{wt}$ ) were significantly different between mothers and neonates $(P<0.0001$, by paired Student's $t$ test or Wilcoxon signed rank test in 724 mother-infant pairs). 
Table 2.2: The polyunsaturated fatty acid composition of maternal plasma phospholipids at delivery $(n=760){ }^{\prime}$

\begin{tabular}{|c|c|c|c|c|c|c|c|c|c|c|c|c|c|c|}
\hline & \multicolumn{7}{|c|}{$m g / L$} & \multicolumn{7}{|c|}{$\% w t / w t$} \\
\hline & \multirow[t]{2}{*}{ Mean } & \multirow[t]{2}{*}{ SD } & \multicolumn{5}{|c|}{ Percentile } & \multirow[t]{2}{*}{ Mean } & \multirow[t]{2}{*}{ SD } & \multicolumn{5}{|c|}{ Percentile } \\
\hline & & & 10 & 25 & 50 & 75 & 90 & & & 10 & 25 & 50 & 75 & 90 \\
\hline \multicolumn{15}{|l|}{ Fatty acid } \\
\hline $18: 2 n-6$ & 361.54 & 76.87 & 262.74 & 310.93 & 359.67 & 410.82 & 460.89 & 20.69 & 2.48 & 17.94 & 19.29 & 20.53 & 22.15 & 23.92 \\
\hline $18: 3 n-6$ & 0.62 & 0.42 & 0.00 & 0.36 & 0.56 & 0.84 & 1.12 & 0.03 & 0.02 & 0.00 & 0.02 & 0.03 & 0.05 & 0.06 \\
\hline $20: 2 n-6$ & 7.77 & 2.05 & 5.23 & 6.46 & 7.63 & 9.09 & 10.31 & 0.44 & 0.08 & 0.35 & 0.39 & 0.44 & 0.49 & 0.54 \\
\hline $20: 3 n-6$ & 61.21 & 17.36 & 40.70 & 49.47 & 59.59 & 70.55 & 83.82 & 3.48 & 0.63 & 2.76 & 3.07 & 3.41 & 3.82 & 4.32 \\
\hline $20: 4 n-6$ & 149.31 & 37.04 & 105.73 & 122.93 & 146.59 & 171.80 & 196.32 & 8.57 & 1.55 & 6.66 & 7.60 & 8.43 & 9.44 & 10.35 \\
\hline $22: 2 n-6$ & 0.33 & 0.20 & 0.00 & 0.23 & 0.34 & 0.45 & 0.57 & 0.02 & 0.01 & 0.00 & 0.01 & 0.02 & 0.03 & 0.03 \\
\hline $22: 4 n-6$ & 6.57 & 1.88 & 4.41 & 5.32 & 6.37 & 7.56 & 9.07 & 0.38 & 0.08 & 0.28 & 0.33 & 0.37 & 0.42 & 0.48 \\
\hline $22: 5 n-6$ & 8.98 & 3.55 & 4.86 & 6.59 & 8.50 & 10.83 & 13.85 & 0.51 & 0.17 & 0.31 & 0.40 & 0.49 & 0.61 & 0.73 \\
\hline $24: 2 n-6$ & 3.02 & 1.17 & 1.73 & 2.23 & 2.86 & 3.61 & 4.53 & 0.18 & 0.07 & 0.10 & 0.13 & 0.17 & 0.21 & 0.26 \\
\hline$\sum n-6$ & 599.57 & 115.56 & 451.45 & 545.61 & 601.88 & 674.56 & 744.59 & 34.31 & 2.13 & 32.00 & 33.12 & 34.38 & 35.64 & 36.67 \\
\hline$\sum \mathrm{n}-6 \mathrm{LCP}$ & 226.06 & 52.06 & 162.26 & 191.22 & 223.07 & 258.58 & 296.03 & 12.94 & 1.76 & 10.89 & 11.85 & 12.88 & 13.94 & 15.00 \\
\hline $18: 3 n-3$ & 3.99 & 4.69 & 1.65 & 2.45 & 3.69 & 4.98 & 6.41 & 0.23 & 0.48 & 0.11 & 0.15 & 0.21 & 0.28 & 0.34 \\
\hline $20: 3 n-3$ & 0.36 & 0.29 & 0.00 & 0.18 & 0.39 & 0.53 & 0.65 & 0.02 & 0.02 & 0.00 & 0.00 & 0.02 & 0.03 & 0.04 \\
\hline $20: 4 n-3$ & 2.40 & 1.17 & 1.13 & 1.60 & 2.18 & 3.02 & 3.97 & 0.14 & 0.06 & 0.07 & 0.10 & 0.12 & 0.17 & 0.21 \\
\hline $20: 5 n-3$ & 6.51 & 5.78 & 2.83 & 3.79 & 5.24 & 7.11 & 10.12 & 0.36 & 0.51 & 0.18 & 0.23 & 0.30 & 0.40 & 0.53 \\
\hline $22: 5 n-3$ & 9.54 & 2.83 & 6.44 & 7.57 & 9.25 & 11.16 & 13.10 & 0.54 & 0.12 & 0.40 & 0.46 & 0.53 & 0.61 & 0.69 \\
\hline $22: 6 n-3$ & 66.79 & 18.65 & 44.58 & 54.18 & 65.16 & 77.66 & 91.11 & 3.83 & 0.78 & 2.91 & 3.29 & 3.76 & 4.26 & 4.90 \\
\hline$\sum n-3$ & 89.34 & 25.13 & 60.42 & 71.85 & 87.35 & 102.75 & 120.81 & 5.12 & 1.26 & 3.99 & 4.42 & 4.99 & 5.65 & 6.32 \\
\hline$\sum n-3$ LCP & 84.98 & 23.77 & 57.54 & 68.29 & 82.84 & 98.23 & 115.63 & 4.87 & 1.03 & 3.76 & 4.17 & 4.77 & 5.37 & 6.08 \\
\hline $20: 3 n-9$ & 4.27 & 2.44 & 1.66 & 2.57 & 3.77 & 5.51 & 7.60 & 0.24 & 0.13 & 0.11 & 0.16 & 0.22 & 0.31 & 0.41 \\
\hline$\sum n-7+n-9$ & 227.71 & 50.40 & 166.52 & 196.08 & 225.34 & 258.71 & 293.25 & 13.02 & 1.49 & 11.28 & 12.07 & 12.99 & 14.04 & 14.82 \\
\hline$\Sigma$ PUFA & 693.53 & 130.91 & 526.35 & 611.44 & 696.97 & 779.59 & 863.54 & 39.70 & 1.54 & 37.77 & 38.73 & 39.72 & 40.73 & 41.64 \\
\hline$\Sigma$ MUFA & 225.02 & 49.45 & 164.79 & 193.47 & 223.15 & 254.74 & 289.50 & 12.87 & 1.45 & 11.17 & 11.95 & 12.85 & 13.83 & 14.59 \\
\hline$\Sigma$ SFA & 798.83 & 146.44 & 614.51 & 701.75 & 795.24 & 891.35 & 989.63 & 45.78 & 1.29 & 44.34 & 45.04 & 45.78 & 46.46 & 47.13 \\
\hline Total fatty acids & 1746.3 & 321.6 & 1338.5 & 1540.3 & 1754.9 & 1953.1 & 2167.6 & 100.00 & & & & & & \\
\hline
\end{tabular}

${ }^{1}$ For legend see previous page. 
Table 2.3: The polyunsaturated fatty acid composition of umbilical cord plasma phospholipids in boys $(n=430){ }^{1}$

\begin{tabular}{|c|c|c|c|c|c|c|c|c|c|c|c|c|c|c|}
\hline & \multicolumn{7}{|c|}{$m g / L$} & \multicolumn{7}{|c|}{$\% w t w t$} \\
\hline & \multirow[t]{2}{*}{ Mean } & \multirow[t]{2}{*}{ SD } & \multicolumn{5}{|c|}{ Percentile } & \multirow[t]{2}{*}{ Mean } & \multirow[t]{2}{*}{ SD } & \multicolumn{5}{|c|}{ Percentile } \\
\hline & & & 10 & 25 & 50 & 75 & 90 & & & 10 & 25 & 50 & 75 & 90 \\
\hline \multicolumn{15}{|l|}{ Fatty acid } \\
\hline $18: 2 n-6$ & $43.46^{2}$ & 13.43 & 29.83 & 35.16 & 41.86 & 49.80 & 57.72 & 7.59 & 1.37 & 6.09 & 6.74 & 7.46 & 8.40 & 9.17 \\
\hline $18: 3 n-6$ & 0.30 & 0.22 & 0.00 & 0.17 & 0.31 & 0.43 & 0.57 & 0.05 & 0.04 & 0.00 & 0.02 & 0.06 & 0.08 & 0.10 \\
\hline $20: 2 n-6$ & $2.08^{d}$ & 0.54 & 1.44 & 1.72 & 2.03 & 2.38 & 2.78 & 0.37 & 0.07 & 0.29 & 0.32 & 0.36 & 0.40 & 0.45 \\
\hline $20: 3 n-6$ & $28.83^{d}$ & 6.99 & 20.92 & 24.07 & 28.19 & 32.48 & 37.88 & 5.08 & 0.86 & 4.00 & 4.48 & 5.03 & 5.56 & 6.28 \\
\hline $20: 4 n-6$ & $94.84^{d}$ & 19.51 & 72.36 & 81.03 & 93.46 & 105.16 & 118.80 & 16.70 & 1.69 & 14.69 & 15.69 & 16.69 & 17.78 & 18.72 \\
\hline $22: 2 n-6$ & 0.23 & 0.15 & 0.00 & 0.14 & 0.24 & 0.32 & 0.41 & 0.04 & 0.03 & 0.00 & 0.02 & 0.04 & 0.06 & 0.07 \\
\hline $22: 4 n-6$ & $4.53^{b}$ & 1.14 & 3.17 & 3.73 & 4.43 & 5.12 & 6.03 & 0.80 & 0.14 & 0.62 & 0.70 & 0.79 & 0.88 & 0.98 \\
\hline $22: 5 n-6$ & 4.77 & 1.80 & 2.68 & 3.38 & 4.62 & 5.81 & 7.24 & 0.84 & 0.27 & 0.49 & 0.63 & 0.81 & 1.01 & 1.20 \\
\hline $24: 2 n-6$ & $3.40^{b}$ & 1.12 & 2.13 & 2.63 & 3.29 & 4.07 & 4.78 & 0.60 & 0.16 & 0.40 & 0.50 & 0.59 & 0.70 & 0.81 \\
\hline$\sum n-6$ & $182.41^{d}$ & 36.00 & 141.65 & 157.81 & 180.87 & 201.77 & 225.00 & 32.06 & 1.69 & 29.93 & 31.03 & 32.13 & 33.25 & 34.07 \\
\hline$\sum \mathrm{n}-6 \mathrm{LCP}$ & $132.96^{d}$ & 25.23 & 103.25 & 115.93 & 131.43 & 147.89 & 164.07 & 23.41 & 1.58 & 21.53 & 22.56 & 23.50 & 24.41 & 25.33 \\
\hline $18: 3 n-3$ & ND & - & - & - & - & - & - & ND & - & - & - & - & - & - \\
\hline $20: 3 n-3$ & ND & - & - & - & - & - & - & ND & - & - & - & - & - & - \\
\hline $20: 4 n-3$ & 0.47 & 0.28 & 0.00 & 0.32 & 0.49 & 0.66 & 0.83 & 0.08 & 0.05 & 0.00 & 0.06 & 0.09 & 0.11 & 0.14 \\
\hline $20: 5 n-3$ & $1.23^{\mathrm{a}}$ & 0.69 & 0.58 & 0.85 & 1.12 & 1.46 & 2.01 & 0.22 & 0.11 & 0.11 & 0.15 & 0.20 & 0.26 & 0.35 \\
\hline $22: 5 n-3$ & $2.61^{\mathrm{a}}$ & 1.14 & 1.38 & 1.79 & 2.42 & 3.16 & 4.06 & 0.46 & 0.17 & 0.26 & 0.33 & 0.43 & 0.56 & 0.69 \\
\hline $22: 6 n-3$ & $34.82^{b}$ & 10.14 & 23.27 & 28.06 & 33.53 & 40.10 & 48.23 & 6.12 & 1.30 & 4.47 & 5.16 & 6.06 & 7.01 & 7.83 \\
\hline$\sum n-3$ & $39.39^{b}$ & 11.50 & 26.64 & 31.67 & 38.08 & 45.18 & 54.98 & 6.93 & 1.47 & 5.04 & 5.77 & 6.88 & 8.04 & 8.93 \\
\hline$\sum n-3$ LCP & $39.13^{b}$ & 11.45 & 26.56 & 31.42 & 37.89 & 45.04 & 54.65 & 6.88 & 1.47 & 5.02 & 5.73 & 6.78 & 7.98 & 8.91 \\
\hline $20: 3 n-9$ & 2.62 & 1.73 & 0.93 & 1.47 & 2.22 & 3.39 & 4.75 & 0.46 & 0.30 & 0.17 & 0.26 & 0.41 & 0.61 & 0.85 \\
\hline$\sum n-7+n-9$ & $70.63^{c}$ & 19.02 & 50.79 & 58.60 & 68.12 & 80.52 & 90.86 & 12.36 & 1.88 & 10.37 & 11.19 & 12.17 & 13.14 & 14.60 \\
\hline$\sum$ PUFA & $224.64^{d}$ & 43.65 & 176.01 & 194.89 & 220.84 & 250.04 & 274.26 & 39.49 & 1.75 & 37.34 & 38.37 & 39.49 & 40.63 & 41.72 \\
\hline$\Sigma$ MUFA & $68.09^{c}$ & 18.15 & 48.89 & 56.89 & 65.46 & 76.84 & 87.08 & 11.92 & 1.70 & 10.12 & 10.82 & 11.76 & 12.67 & 13.75 \\
\hline $\bar{\Sigma}$ SFA & $270.56^{d}$ & 52.37 & 211.07 & 234.51 & 267.79 & 302.46 & 331.72 & 47.53 & 1.58 & 45.79 & 46.73 & 47.60 & 48.45 & 49.26 \\
\hline Total fatty acids & $569.43^{d}$ & 110.02 & 447.16 & 489.48 & 562.20 & 631.83 & 701.28 & 100.00 & & & & & & \\
\hline
\end{tabular}

${ }^{7}$ For legend see Table 2.2. ${ }^{\mathrm{a}} \mathrm{P}<0.05,{ }^{\mathrm{b}} \mathrm{P}<0.01,{ }^{\mathrm{c}} \mathrm{P}<0.001,{ }^{\mathrm{d}} \mathrm{P}<0.0001$ for difference between boys and girls (two-tailed Student $t$ tests). 
Table 2.4: The polyunsaturated fatty acid composition of umbilical plasma phospholipids in girls $(n=350)$.'

\begin{tabular}{|c|c|c|c|c|c|c|c|c|c|c|c|c|c|c|}
\hline & \multicolumn{7}{|c|}{$m g / L$} & \multicolumn{7}{|c|}{$\% w t / w t$} \\
\hline & \multirow[t]{2}{*}{ Mean } & \multirow[t]{2}{*}{ SD } & \multicolumn{5}{|c|}{ Percentile } & \multirow[t]{2}{*}{ Mean } & \multirow[t]{2}{*}{ SD } & \multicolumn{5}{|c|}{ Percentile } \\
\hline & & & 10 & 25 & 50 & 75 & 90 & & & 10 & 25 & 50 & 75 & 90 \\
\hline \multicolumn{15}{|l|}{ Fatty acid } \\
\hline $18: 2 n-6$ & 46.29 & 22.81 & 31.89 & 37.53 & 43.87 & 50.90 & 61.29 & 7.44 & 1.39 & 6.11 & 6.63 & 7.34 & 8.27 & 8.86 \\
\hline $18: 3 n-6$ & 0.31 & 0.23 & 0.00 & 0.16 & 0.31 & 0.44 & 0.59 & 0.05 & 0.04 & 0.00 & 0.03 & 0.05 & 0.07 & 0.09 \\
\hline $20: 2 n-6$ & 2.29 & 0.74 & 1.52 & 1.82 & 2.19 & 2.61 & 3.15 & 0.37 & 0.07 & 0.29 & 0.32 & 0.37 & 0.41 & 0.45 \\
\hline $20: 3 n-6$ & 31.67 & 7.85 & 22.53 & 26.24 & 31.26 & 35.85 & 42.35 & 5.18 & 0.85 & 4.17 & 4.60 & 5.08 & 5.71 & 6.24 \\
\hline $20: 4 n-6$ & 103.20 & 22.89 & 77.05 & 87.55 & 101.78 & 114.45 & 134.44 & 16.84 & 1.61 & 14.92 & 15.80 & 16.69 & 17.90 & 18.87 \\
\hline $22: 2 n-6$ & 0.24 & 0.16 & 0.00 & 0.13 & 0.25 & 0.33 & 0.44 & 0.04 & 0.03 & 0.00 & 0.02 & 0.04 & 0.05 & 0.07 \\
\hline $22: 4 n-6$ & 4.78 & 1.29 & 3.31 & 3.87 & 4.63 & 5.41 & 6.55 & 0.78 & 0.14 & 0.61 & 0.68 & 0.77 & 0.87 & 0.96 \\
\hline $22: 5 n-6$ & 4.99 & 1.93 & 2.75 & 3.64 & 4.82 & 6.06 & 7.28 & 0.81 & 0.26 & 0.51 & 0.64 & 0.78 & 0.94 & 1.16 \\
\hline $24: 2 n-6$ & 3.68 & 1.26 & 2.23 & 2.80 & 3.55 & 4.41 & 5.25 & 0.60 & 0.16 & 0.40 & 0.49 & 0.60 & 0.71 & 0.78 \\
\hline$\sum n-6$ & 197.42 & 47.89 & 151.55 & 168.74 & 194.70 & 218.88 & 246.46 & 32.10 & 1.69 & 29.87 & 30.94 & 32.13 & 33.25 & 34.13 \\
\hline$\sum n-6 L C P$ & 144.63 & 30.04 & 111.10 & 124.05 & 142.63 & 161.99 & 182.37 & 23.61 & 1.54 & 21.74 & 22.77 & 23.63 & 24.58 & 25.46 \\
\hline $18: 3 n-3$ & ND & - & - & - & - & - & - & ND & - & - & - & - & - & - \\
\hline $20: 3 n-3$ & ND & - & - & - & - & - & - & ND & - & - & - & - & - & - \\
\hline $20: 4 n-3$ & 0.51 & 0.34 & 0.00 & 0.34 & 0.52 & 0.71 & 0.88 & 0.08 & 0.05 & 0.00 & 0.06 & 0.09 & 0.11 & 0.14 \\
\hline $20: 5 n-3$ & 1.31 & 0.70 & 0.61 & 0.86 & 1.20 & 1.68 & 2.16 & 0.22 & 0.11 & 0.10 & 0.15 & 0.20 & 0.28 & 0.34 \\
\hline $22: 5 n-3$ & 2.80 & 1.21 & 1.46 & 1.96 & 2.59 & 3.47 & 4.17 & 0.46 & 0.17 & 0.26 & 0.33 & 0.44 & 0.57 & 0.69 \\
\hline $22: 6 n-3$ & 37.17 & 11.01 & 25.30 & 29.25 & 35.35 & 41.86 & 53.14 & 6.08 & 1.36 & 4.35 & 5.03 & 5.98 & 7.04 & 8.04 \\
\hline$\sum n-3$ & 42.03 & 12.49 & 28.42 & 33.23 & 39.69 & 47.86 & 59.64 & 6.88 & 1.53 & 4.91 & 5.71 & 6.81 & 7.94 & 9.08 \\
\hline$\sum \mathrm{n}-3 \mathrm{LCP}$ & 41.79 & 12.38 & 28.34 & 33.02 & 39.49 & 47.51 & 59.64 & 6.84 & 1.53 & 4.90 & 5.68 & 6.75 & 7.92 & 9.03 \\
\hline $20: 3 n-9$ & 2.66 & 1.64 & 1.01 & 1.56 & 2.28 & 3.30 & 5.13 & 0.44 & 0.25 & 0.17 & 0.26 & 0.38 & 0.56 & 0.77 \\
\hline$\sum n-7+n-9$ & 75.35 & 22.38 & 53.42 & 62.97 & 72.89 & 84.90 & 97.29 & 12.33 & 1.91 & 10.40 & 11.12 & 12.06 & 13.17 & 14.47 \\
\hline$\Sigma$ PUFA & 242.40 & 55.59 & 187.48 & 207.54 & 238.75 & 270.35 & 302.03 & 39.47 & 1.72 & 37.31 & 38.46 & 39.49 & 40.67 & 41.50 \\
\hline$\Sigma$ MUFA & 73.35 & 22.30 & 51.78 & 61.13 & 70.56 & 81.82 & 93.66 & 11.88 & 1.74 & 10.13 & 10.82 & 11.66 & 12.62 & 13.66 \\
\hline$\Sigma \mathrm{SFA}$ & 292.65 & 66.38 & 222.53 & 250.61 & 286.86 & 322.77 & 363.90 & 47.62 & 1.24 & 46.23 & 46.81 & 47.72 & 48.41 & 49.07 \\
\hline Total fatty acids & 614.82 & 141.50 & 469.69 & 525.02 & 603.68 & 683.44 & 760.93 & 100.00 & & & & & & \\
\hline
\end{tabular}

${ }^{\mathrm{T}}$ For legend see Table $\mathbf{2 . 2}$. 
Table 2.5: Concentrations of saturated, $n-7$ and n-9 unsaturated, and trans fatty acids in maternal and umbilical cord plasma phospholipids.

\begin{tabular}{|c|c|c|c|c|}
\hline \multirow[t]{2}{*}{ Fatty acid } & \multicolumn{2}{|c|}{ Maternal plasma } & \multicolumn{2}{|c|}{ Umbilical plasma } \\
\hline & $m g / L$ & $\% w t / w t$ & $m g / L$ & $\% w t / w t$ \\
\hline $14: 0$ & $5.20 \pm 1.88$ & $0.29 \pm 0.08$ & $1.41 \pm 0.59$ & $0.24 \pm 0.08$ \\
\hline $15: 0$ & $2.97 \pm 0.99$ & $0.17 \pm 0.04$ & $0.67 \pm 0.31$ & $0.11 \pm 0.05$ \\
\hline $16: 0$ & $543.16 \pm 102.12$ & $30.96 \pm 1.21$ & $163.93 \pm 34.17$ & $27.69 \pm 1.42$ \\
\hline $17: 0$ & $4.84 \pm 1.77$ & $0.28 \pm 0.09$ & $1.56 \pm 0.70$ & $0.27 \pm 0.11$ \\
\hline 18:0 & $168.48 \pm 33.53$ & $9.61 \pm 0.85$ & $84.58 \pm 17.22$ & $14.31 \pm 1.04$ \\
\hline $20: 0$ & $9.14 \pm 1.99$ & $0.52 \pm 0.09$ & $5.09 \pm 1.43$ & $0.86 \pm 0.16$ \\
\hline $22: 0$ & $26.04 \pm 6.18$ & $1.49 \pm 0.29$ & $8.93 \pm 2.84$ & $1.51 \pm 0.37$ \\
\hline $23: 0$ & $9.79 \pm 2.38$ & $0.56 \pm 0.10$ & $1.23 \pm 0.51$ & $0.21 \pm 0.07$ \\
\hline $24: 0$ & $16.46 \pm 4.20$ & $0.94 \pm 0.19$ & $8.95 \pm 2.49$ & $1.51 \pm 0.29$ \\
\hline $16: \ln -7$ & $8.24 \pm 4.25$ & $0.46 \pm 0.21$ & $3.05 \pm 1.52$ & $0.51 \pm 0.20$ \\
\hline $18: \ln -7$ & $25.46 \pm 8.85$ & $1.46 \pm 0.44$ & $14.13 \pm 3.92$ & $2.38 \pm 0.39$ \\
\hline $20: \ln -7$ & ND & ND & ND & ND \\
\hline $18: \ln -9$ & $154.22 \pm 36.21$ & $8.78 \pm 1.20$ & $39.58 \pm 12.79$ & $6.66 \pm 1.32$ \\
\hline $18: 2 n-9$ & ND & ND & ND & ND \\
\hline $20: \ln -9$ & $2.49 \pm 0.69$ & $0.14 \pm 0.03$ & $0.31 \pm 0.18$ & $0.05 \pm 0.03$ \\
\hline $22: \ln -9$ & ND & ND & ND & ND \\
\hline $22: 3 n-9$ & ND & ND & ND & ND \\
\hline $24: \ln -9$ & $28.48 \pm 7.40$ & $1.63 \pm 0.35$ & $13.14 \pm 3.67$ & $2.21 \pm 0.38$ \\
\hline ¿Trans & $5.42 \pm 4.16$ & $0.31 \pm 0.23$ & $0.40 \pm 0.51$ & $0.07 \pm 0.08$ \\
\hline
\end{tabular}

Mean \pm SD. ND, not detectable or detected in trace amounts only.

The ratio of the sum of all essential fatty acids and their derivatives to the sum of all non-essential fatty acids of the $n-7$ and $n-9$ families $\left(\sum n-3+n-6 / \sum n-7+n-9\right)$ and the ratio of all $n-3$ fatty acids to all $n-6$ fatty acids $\left(\sum n-3 / \sum n-6\right)$ were positively related to the length of gestation $(P<0.0001$ for both $)$. The distribution of the relative concentrations of arachidonic acid (20:4n-6) and docosahexaenoic acid (22:6n-3) in umbilical plasma phospholipids are plotted as a function of gestational age (Figure 2.1).

The maternal concentration (\% wt $/ \mathrm{wt})$ of docosahexaenoic acid at delivery was dependent on maternal parity at study entry. Mean \pm SD 22:6n-3 concentration was 3.8 $\pm 0.8,3.7 \pm 0.8$, and $3.6 \pm 0.9 \% \mathrm{wt} / \mathrm{wt}(P<0.05$ by ANOVA) for nullipara, primipara, and multipara, respectively. Umbilical cord plasma concentrations of 22:6n-3 did not relate to maternal parity at study entry. Also, we did not observe a significant relationship between parity and maternal plasma and umbilical cord plasma concentrations of arachidonic acid (20:4n-6).

The average dietary fatty acid intake, according to the dietary history in a subsample of 288 women midway in gestation, is given in Table 2.1. The average intake of linoleic acid $(18: 2 n-6)$ in this population of pregnant women was approximately $85 \%$ of total PUFA intake. The relationship between maternal 18:2n-6 intake and the PUFA concentrations in maternal and umbilical plasma phospholipids are shown in Table 2.6. Higher intake of $18: 2 \mathrm{n}-6$ was associated with higher concentrations ( $\% \mathrm{wt} / \mathrm{wt})$ of 
$18: 2 n-6$ and lower concentrations (\%wt/wt) of $22: 6 n-3$ in maternal and umbilical plasma phospholipids. The proportion of infants with a $22: 6 n-3$ concentration ( $\%$ $\mathrm{wt} / \mathrm{wt}$ ) below the $10^{\text {th }}$ percentile (appropriate for gestational age) was $3 \%, 10 \%, 11 \%$, and $22 \%$ for mothers in the $1^{\text {st }}, 2^{\text {nd }}, 3^{\text {rd }}$, and $4^{\text {th }}$ quartile of linoleic acid intake, respectively. A higher intake of $18: 2 n-6$ did not relate to higher $20: 4 n-6$ concentrations.

A higher intake of PUFAs other than linoleic acid (mostly $\alpha$-linolenic acid) was related to higher concentrations of $\alpha$-linolenic acid (18:3n-3) in maternal plasma and to higher concentrations of its derivative eicosapentaenoic acid (20:5n-3) in umbilical cord plasma (not shown). The higher intake of PUFAs other than linoleic acid did not relate to higher 22:6n-3 concentrations in maternal or umbilical cord blood. There was a positive relation between the intake of linoleic acid and the intake of other PUFAs ( $r$ $=0.50, P<0.0001)$.

\section{Discussion}

The PUFA composition of maternal plasma phospholipids changes during pregnancy. This was described by $\mathrm{Al}$ et al. ${ }^{6}$ The PUFA concentration in maternal plasma lipids is known to be a strong predictor of the PUFA concentration in umbilical plasma lipids. ${ }^{5,6}$ Both are influenced by the maternal dietary intake of fatty acids (Table 2.6). ${ }^{13,21-24}$ Umbilical fatty acid concentrations measured after delivery, as in the present study, are essentially similar to concentrations found in fetal plasma when measured at a comparable gestational age. ${ }^{25}$ Therefore, they seem to be a reasonable reflection of the intrauterine fatty acid supply. During intrauterine life, the placenta plays a crucial role in the delivery of essential fatty acids and their derivatives to the fetus. ${ }^{26,27}$ A selective transfer of LCPs seems to occur. As a result, the proportion (but not the absolute concentration) of LCPs is higher and the proportion of their parent essential fatty acids is lower in fetal and umbilical samples, compared with the corresponding proportion in maternal plasma (Tables 2.2-2.4). ${ }^{5.25}$ Similar findings were obtained when studying the fatty acid composition of plasma cholesterol esters, triglycerides and total lipids. ${ }^{8-11}$ During postnatal life, the essential fatty acids and their derivatives are obtained from the infant's diet (initially from the breast milk or artificial formula). As a result, the fatty acid composition of plasma phospholipids will change and ultimately reach a pattern similar to that found in adults. ${ }^{9.26}$

As stated above, the PUFA concentrations in umbilical plasma are related to the PUFA levels in maternal plasma. ${ }^{5.6}$ In addition, the PUFA composition of umbilical plasma phospholipids is also dependent on the duration of pregnancy (the present paper). ${ }^{25.28 .29}$ Most pronounced is the increased absolute and relative concentration of docosahexaenoic acid (22:6n-3) (Figure 2.1). ${ }^{28}$ This has also been described in fetal plasma phospholipids during pregnancy ${ }^{25}$ and in neonatal plasma cholesteryl esters ${ }^{10}$ and triglycerides. ${ }^{11}$ 

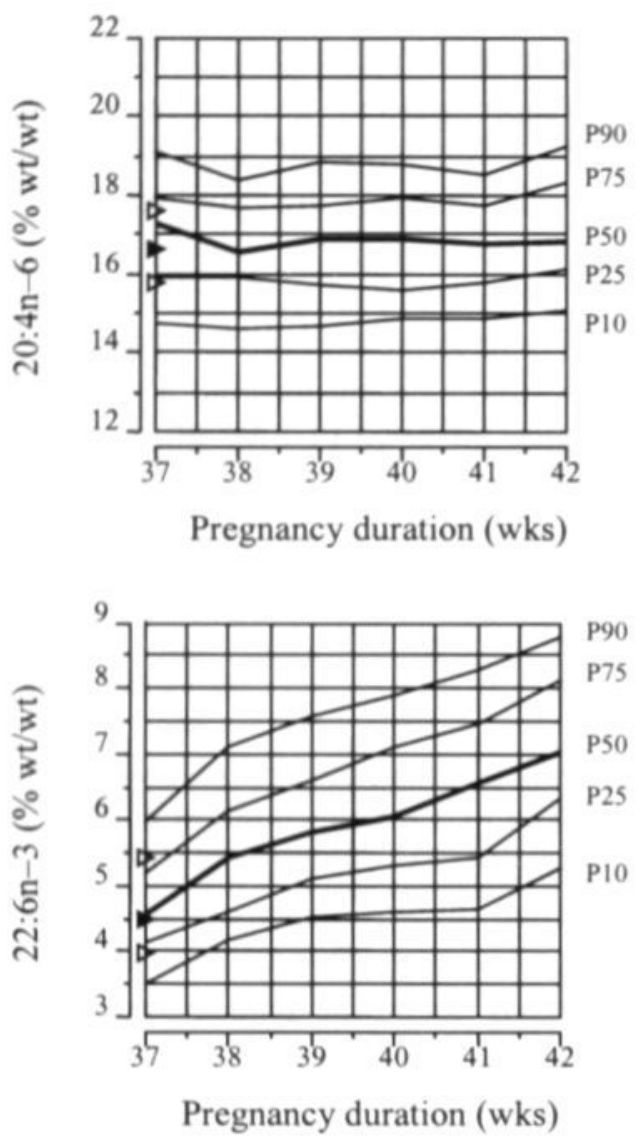

Figure 2.1: Distribution of the umbilical cord plasma phospholipid concentration of arachidonic acid $(20: 4 n-6)$ and docosa-hexaenoic acid $(22: 6 n-3)$ as a function of gestational age at birth. The number of iniants in each gestational age group is given underneath the figure. The lines represent the $10^{\text {th }}, 25^{\text {th }}$, $50^{\text {th }}, 75^{\text {th }}$ and $90^{\text {th }}$ percentiles $(\mathrm{P})$. The triangles represent the $25^{\text {th }}, 50^{\text {th }}$ and $75^{\text {th }}$ percentiles for the preterm infants who were excluded only because of premature birth (average gestational age at birth: 35 $\pm 1.5 \mathrm{wk}, n=39) . P<0.0001$ for the association between docosahexaenoic acid concentration and gestational age at birth (Spearman rank correlation test). The ararchidonic acid concentration was not significantly related to the length of gestation. For more plots see Appendix $G$.

A preference for transport of $22: 6 n-3$ to the fetal circulation by the placenta might explain these observations. ${ }^{30}$ Although only limited information is available, an increase in fetal 22:6n-3 biosynthesis does not seem likely because stable fetal liver delta- 5 and delta- 6 desaturase activities have been found throughout the third trimester. $^{31}$ 
Table 2.6: Average n-3 and n-6 polyunsaturated fatty acid concentrations in maternal and umbilical plasma phospholipids at delivery according to the maternal linoleic acid $(18: 2 n-6)$ intake at midgestation ( $\mathrm{g} /$ day).

\begin{tabular}{|c|c|c|c|c|}
\hline \multirow[t]{2}{*}{ Fatty acid $(\mathrm{mg} / \mathrm{L})$} & \multicolumn{4}{|c|}{ Quartiles of linoleic acid intake } \\
\hline & I & II & III & IV \\
\hline \multicolumn{5}{|l|}{ Maternal plasma } \\
\hline $18: 2 n-6^{2}$ & 342.89 & 376.37 & 382.73 & 383.94 \\
\hline $20: 3 n-6$ & 61.23 & 64.75 & 62.25 & 59.49 \\
\hline $20: 4 n-6$ & 141.70 & 155.78 & 142.72 & 146.02 \\
\hline $18: 3 n-3$ & 3.69 & 3.93 & 4.15 & 3.40 \\
\hline $20: 5 n-3^{2}$ & 6.33 & 5.99 & 6.02 & 5.25 \\
\hline $22: 5 n-3^{2}$ & 9.77 & 10.38 & 9.74 & 8.84 \\
\hline $22: 6 n-3^{2}$ & 70.16 & 71.25 & 65.21 & 64.39 \\
\hline \multicolumn{5}{|l|}{ Umbilical plasma } \\
\hline $18: 2 n-6$ & 45.75 & 41.87 & 43.31 & 48.19 \\
\hline $20: 3 n-6$ & 31.38 & 29.45 & 30.48 & 30.03 \\
\hline $20: 4 n-6$ & 100.27 & 95.60 & 92.14 & 95.29 \\
\hline $20: 5 n-3^{b}$ & 1.52 & 1.32 & 1.19 & 1.26 \\
\hline $22: 5 n-3^{b}$ & 2.96 & 2.66 & 2.34 & 2.58 \\
\hline $22: 6 n-3^{c}$ & 40.27 & 35.55 & 33.34 & 33.48 \\
\hline \multirow[t]{2}{*}{ Fatty acid $(\% w t / w t)$} & \multicolumn{4}{|c|}{ Quartiles of linoleic acid intake } \\
\hline & I & II & III & IV \\
\hline \multicolumn{5}{|l|}{ Maternal plasma } \\
\hline $18: 2 n-6^{d}$ & 20.04 & 20.51 & 21.58 & 21.89 \\
\hline $20: 3 n-6^{2}$ & 3.57 & 3.50 & 3.46 & 3.35 \\
\hline $20: 4 n-6$ & 8.29 & 8.52 & 8.02 & 8.37 \\
\hline $18: 3 n-3$ & 0.21 & 0.21 & 0.23 & 0.21 \\
\hline $20: 5 n-3^{a}$ & 0.37 & 0.32 & 0.33 & 0.31 \\
\hline $22: 5 n-3^{b}$ & 0.57 & 0.56 & 0.54 & 0.51 \\
\hline $22: 6 n-3^{b}$ & 4.11 & 3.88 & 3.66 & 3.69 \\
\hline \multicolumn{5}{|l|}{ Umbilical plasma } \\
\hline $18: 2 n-6^{c}$ & 7.34 & 7.28 & 7.73 & 8.11 \\
\hline $20: 3 n-6$ & 5.07 & 5.12 & 5.43 & 5.14 \\
\hline $20: 4 n-6$ & 16.28 & 16.60 & 16.48 & 16.27 \\
\hline $20: 5 n-3^{2}$ & 0.25 & 0.23 & 0.22 & 0.22 \\
\hline $22: 5 n-3$ & 0.48 & 0.47 & 0.42 & 0.44 \\
\hline $22: 6 n-3^{b}$ & 6.51 & 6.21 & 5.93 & 5.73 \\
\hline
\end{tabular}

The average $( \pm$ SE) linoleic acid intake was $7.57 \pm 0.22,12.34 \pm 0.14,16.79 \pm 0.17$, and $25.71 \pm 0.72$ $\mathrm{g} /$ day, for the quartiles I, II, III and IV respectively.

${ }^{a} P<0.05,{ }^{b} P<0.01,{ }^{c} P<0.001,{ }^{d} P<0.0001$ for relation with linoleic acid intake (Sperrman rank correlation test between the continuos variables, $n=224$ for maternal plasma and $n=233$ for umbilical plasma).

Uauy et al. ${ }^{32}$ even reported decreased LCP synthesis rates in infants born after a longer duration of pregnancy. The PUFA composition of umbilical plasma phospholipids is also related to the infant size at birth. ${ }^{29} \mathrm{~A}$ higher weight at birth is associated with lower 
proportions of both arachidonic acid (20:4n-6) and docosahexaenoic acid (22:6n-3) in umbilical plasma phospholipids of term neonates (see Chapter 3). ${ }^{29}$ Moreover, the concentrations of eicosatrienoic acid (20:3n-9) are higher and the ratio $\sum n-3+n-6 / \sum n-$ $7+n-9$ and the ratio $22: 6 n-3 / 22: 5 n-6$ are lower in heavier infants, ${ }^{29}$ indicating a lower biochemical essential fatty acid status. ${ }^{33-35}$ These observations suggest that (under the present dietary conditions) the maternal-to-fetal delivery of LCPs may not fully keep up with fetal accretion rate in larger infants. Previously, contrasting findings had been described in smaller samples of premature infants and low-birth-weight neonates. ${ }^{26,36-38}$

Apart from the aspects discussed above, several other factors should be taken into consideration when the information reported in this paper is used. First, the comparability of fatty acid concentrations between laboratories is limited by differences in the analytical procedures. Especially the time of addition of the internal standard is important since this influences the absolute concentration $(\mathrm{mg} / \mathrm{L}){ }^{39}$ Secondly, differences in dietary habits between populations are likely to be the reason for differences in the distribution of fatty acid concentrations. Therefore, the information presented here may be applicable only to populations with comparable dietary habits. Considering the high average intake of linoleic acid in our population (a characteristic feature of the Western-style diet) and the putative beneficial effects of a higher (n-3) LCP intake, ${ }^{40,41}$ it is questionable whether the percentiles given in this paper should be regarded as a pursuable standard. As shown in Table 2.6, higher intake of linoleic acid is related to lower maternal and umbilical $22: 6 n-3$ concentrations.

Since the LCP status at birth is an important predictor of the postnatal LCP status, ${ }^{38}$ an appropriate prenatal supply of LCPs (as determined by e.g. maternal dietary habits, maternal circulating LCP concentrations and the length of gestation) is important. ${ }^{42}$ The studies on premature infants (who have a low LCP status at birth) show that LCP-enriched formulas may have beneficial effects on the postnatal development. ${ }^{43}$ The findings in term neonates have been less consistent. ${ }^{44}$ Among the variables that may explain this inconsistency could be the LCP status at birth. ${ }^{42}$ Probably, term neonates with a relatively low LCP status at birth will benefit most from LCP-enriched formulas. The information reported in this paper may aid in identifying such infants.

\section{References}

1. Innes SM. Essential fatty acids in growth and development. Prog Lipid Res. 1991;30:39-103.

2. Sattar N, Berry C, Greer IA. Essential fatty acids in relation to pregnancy complications and fetal development. Br J Obstet Gynaecol. 1998;105:1248-1255.

3. Hamosh M, Salem N. Long-chain polyunsaturated fatty acids. Biol Neonate. 1998;74:106-120.

4. Gibson RA, Makrides M. n-3 Polyunsaturated fatty acid requirements of term infants. Am J Clin Nutr. 2000:71:251S-255S.

5. Al MD, Hornstra G, Schouw YTvd, Bulstra-Ramakers MT, Huisjes HJ. Biochemical EFA status of mothers and their neonates after normal pregnancy. Early Hum Dev. 1990;24:239-48. 
6. Al MD, Houwelingen ACv, Kester AD, Hasaart TH, de Jong AE, Hornstra G. Maternal essential fatty acid patterns during normal pregnancy and their relationship to the neonatal essential fatty acid status. Br J Nutr. 1995;74:55-68.

7. Sanjurjo P, Matorras R, Ingunza N, Alonso M, Rodriguez-Alarcon J, Perteagudo L. Crosssectional study of percentual changes in total plasmatic fatty acids during pregnancy. Horm Metab Res. 1993;25:590-592.

8. Koletzko B, Müller J. Cis- and trans-isomeric fatty acids in plasma of newborn infants and their mothers. Biol Neonate. 1990;57:172-178.

9. Decsi T, Koletzko B. Fatty acid composition of plasma lipids in healthy subjects from birth to young adulthood. Eur J Pediatr. 1994;153:520-525.

10. Hoving ED, Beusekom CMv, Nijboer HJ, Muskiet FAJ. Gestational age dependency of essential fatty acids in cord plasma cholesterol esters and triglycerides. Pediatr Res. 1994;35:461-469.

11. Berghaus TM, Demmelmair H, Koletzko B. Essential fatty acids and their long-chain polyunsaturated metabolites in maternal and cord plasma triglycerides during late gestation. Biol Neonate. 2000;77:96-100.

12. Al MD, Houwelingen ACv, Badart-Smook A, Hasaart TH, Roumen FJ, Hornstra G. The essential fatty acid status of mother and child in pregnancy-induced hypertension: a prospective longitudinal study. Am J Obstet Gynecol. 1995;172:1605-14.

13. Al MD, Badart-Smook A, Houwelingen ACv, Hasaart TH, Hornstra G. Fat intake of women during normal pregnancy: relationship with maternal and neonatal essential fatty acid status. $J$ Am Coll Nutr. 1996;15:49-55.

14. Kloosterman GJ. On intrauterine growth: The significance of prenatal care. Int $J$ Gyneacol Obstet. 1970;8:895-912.

15. Badart-Smook A, Houwelingen ACv, Al MDM, Kester ADM, Hornstra G. Fetal growth is associated positively with maternal intake of riboflavin and negatively with maternal intake of linoleic acid. J Am Diet Assoc. 1997;97:867-870.

16. Folch J, Lees M, Sloane-Stanley GH. A simple method for the isolation and purification of 'otal lipids from animal tissues. J Biol Chem. 1957;226:497-509.

17. Hoving EB, Jansen G, Volmer M, Doormaal JJv, Muskiet FAJ. Profiling of plasma triglyceride fatty acids as their methylesters by capillary gas chromatography, preceded by a rapid aminopropyl silica column chromatographic separation of lipid classes. J Chromatogr. 1988;434:395-409.

18. Kaluzny MA, Duncan LA, Merritt MV, Epps DE. Rapid separation of lipid classes in high yield and purity using bonded phase columns. J Lipid Res. 1985;26:135-140.

19. Morrisson WR, Smith LM. Preparation of fatty acid methyl esters and demethylacatals from lipids with boron fluoride methanol. J Lipid Res. 1964;5:600-8.

20. Volmer M, Meiborg G, Muskiet AJ. Simultaneous cappillary gas chromatographic profiling of medium- and long-chain fatty acids methyl esters with split injection. $J$ Chromatogr. 1988;434:385-394.

21. Houwelingen ACv, Dalby-Sorensen J, Hornstra G, Simonis MMG, Boris J, Olson S, Secher NJ. Essential fatty acid status of mothers and their babies after fish oil supplementation during pregnancy. Br J Nutr. 1995;74:723-731.

22. Al MD, Houwelingen ACv, Badart-Smook A, Hornstra G. Some aspects of neonatal essential fatty acid status are altered by linoleic acid supplementation of women during pregnancy. $J$ Nutr. 1995;125:2822-30.

23. Bjerve KS, Brubakk AM, Fougner KJ, Johnson H, Midthjell K, Vik T. Omega-3 fatty acids: essential fatty acids with important biological effects, and serum phospholipid fatty acids as markers of dietary $\omega 3$-fatty acid intake. Am J Clin Nutr. 1993;57:801S-806S. 
24. Sanjurjo P, Matorras R, Ingunza N, Rodrigeuz-Alarcón J, Perteagudo L. Blue fish intake and percentual levels of polyunsaturated fatty acids at labor in the mother and newborn infant. Perinat Med. 1994;22:337-344.

25. Houwelingen ACv, Foreman-van Drongelen MMHP, Nicolini U, Nicolaides KH, Al MDM, Kester ADM, Hornstra G. Essential fatty acid status of fetal plasma phospholipids: similar to postnatal values obtained at comparable gestational ages. Early Hum Dev. 1996;46:141-152.

26. Crawford MA. Placental delivery of arachidonic and docosahexaenoic acids: implications for the lipid nutrition of preterm infants. Am J Clin Nutr. 2000;71:275S-284S.

27. Dutta-Roy AK. Transport mechanisms for long-chain polyunsaturated fatty acids in the human placenta. Am J Clin Nutr. 2000;71:315S-322S.

28. Al MDM, Houwelingen ACv, Hornstra G. Long-chain polyunsaturated fatty acids, pregnancy, and pregnancy outcome. Am J Clin Nutr. 2000;71:285S-291S.

29. Rump P, Mensink RP, Kester ADM, Hornstra G. Essential fatty acid composition of plasma phospholipids and birth weight: a study in term neonates. Am J Clin Nutr. 2001;73:797-806.

30. Haggarty P, Ashton J, Joynson M, Abramovich DR, Page K. Effect of maternal polyunsaturated fatty acid concentration on transport by the human placenta. Biol Neonate. 1999;75:350-359.

31. Rodriguez A, Sarda P, Nessmann C, Boulot P, Poisson JP, Leger CL, Descomps B. Fatty acid desaturase activities and polyunsaturated fatty acid composition in human liver between the seventeeth and thirty-sixth gestational weeks. Am J Obstet Gynecol. 1998;179:1063-1070.

32. Uauy R, Mena P, Wegher B, Nieto S, Salem N. Long chain polyunsaturated fatty acid formation in neonates: effect of gestational age and intrauterine growth. Pediatr Res. 2000;47:127-135.

33. Holman RT. Essential fatty acid deficiency in humans. Dietary lipids and postnatal development. New York: Raven; 1973.

34. Hornstra G, Al MDM, Houwelingen ACv, Foreman-van Drongelen MMHP. Essential fatty acids in pregnancy and early human development. Eur J Obstet Gynecol Reprod Biol. 1995;61:57-62.

35. Hoffman DR, Uauy R. Essentiality of dietary omega-3 fatty acids for premature infants; plasma and red blood cell fatty acid composition. Lipids, 1992;27:886-895.

36. Koletzko B, Braun M. Arachidonic acid and early human growth: is there a relation? Ann Nutr Metab. 1991;35:128-131.

37. Leaf AA, Leighfield MJ, Costeloe KL, Crawford MA. Long chain polyunsaturated fatty acids and fetal growth. Early Hum Dev. 1992;30:183-191.

38. Foreman-van Drongelen MMHP, Houwelingen ACv, Kester ADM, Hasaart THM, Blanco CE, Hornstra G. Long-chain polyunsaturated fatty acids in pre-term infants: status at birth and its influence on postnatal levels. J Pediatr. 1995;126:611-618.

39. Foreman-van Drongelen MMHP, Houwelingen ACv, Kester ADM, Jong AEPd, Blanco CE, Hasaart THM, Hornstra G. Long-chain polyene status of preterm infants with regard to the fatty acid composition of their diet: comparison between absolute and relative fatty acid amounts in plasma and red blood cell phospholipids. Br J Nutr. 1995;73:405-422.

40. Uauy R, Mena P, Valenzuela A. Essential fatty acids as determinants of lipid requirements in infants, children and adults. Eur J Clin Nutr. 1999;53:S66-S77.

41. Makrides M, Gibson RA. Long-chain polyunsaturated fatty acid requirements during pregnancy and lactation. Am J Clin Nutr. 2000;71:307S-311S.

42. Innes SM. Maternal diet, length of gestation, and long-chain polyunsaturated fatty acid status of infants at birth. Am J Clin Nutr. 1999;70:181-182.

43. SanGiovanni JP, Parra-Cabrera S, Colditz G, Berkey CS, Dwyer JT. Meta-analysis of dietary essential fatty acids and long-chain polyunsatureted fatty acids as they relate to visual resolution acuity in healthy preterm infants. Pediatrics. 2000;105:1292-1298.

44. SanGiovanny JP, Berkey CS, Dwyer JT, Colditz GA. Dietary essential fatty acids, long-chain polyunsaturated fatty acids, and visual resolution acuity in healthy fullterm infants: a systematic review. Early Hum Dev. 2000;57:165-188. 
Chapter 3 | Essential fatty acid composition of plasma phospholipids and birth weight: a study in term neonates

P. Rump, R.P. Mensink, A.D.M. Kester, and G. Hornstra

Am J Clin Nutr 2001;73:797-806 


\begin{abstract}
Background Essential fatty acids (EFAs) in umbilical cord blood samples are associated with attained birth weight in premature infants and low-birth-weight neonates.

Objective The objective was to investigate relations between the EFA composition of cord and maternal plasma phospholipids and birth weight in term neonates.

Design This was a cross-sectional study in 627 singletons born at term. The plasma phospholipid EFA composition of the mothers was determined by liquid-gas chromatography at study entry ( $\leq 16$ weeks gestation), at delivery, and in cord plasma at birth. Birth weights were normalized to SD scores.

Results In cord plasma, the dihomo- $\gamma$-linolenic acid concentration was positively related to weight SD scores. Both arachidonic acid (AA) and docosahexaenoic acid (DHA) were negatively related to weight SD scores. EFA-status indicators showed similar negative associations, whereas eicosatrienoic acid concentrations were positively related to neonatal size. In maternal plasma, proportions of $n-3$ long-chain polyenes (LCPs) and n-6 LCPs decreased during pregnancy. Larger decreases in AA, DHA, n-3 LCP and n-6 LCP fractions were observed in mothers of heavier babies. Higher concentrations of LCPs in maternal plasma were, however, not related to a larger infant size at birth.

Conclusions A lower biochemical EFA status in umbilical cord plasma and a larger decrease in maternal plasma LCP concentrations are associated with a higher weightfor-gestational-age at birth in term neonates. Our findings do not support a growthstimulating effect of AA or DHA; however, they do suggest that maternal-to-fetal transfer of EFAs might be a limiting factor in determining neonatal EFA status.
\end{abstract}




\section{Introduction}

Growth retardation is one of the prominent features of essential fatty acid (EFA) deficiency in both animals 1,2 and humans. ${ }^{3,4}$ Linoleic acid $(18: 2 \mathrm{n}-6)$ and the longerchain polyunsaturated fatty acids (PUFAs) of the n-6 family are believed to be important for optimal growth. Lower concentrations of EFAs were found in red blood cell membranes, plasma phospholipids, and in the walls of the umbilical artery of lowbirth-weight neonates. ${ }^{5}$ In premature infants, birth weight was positively associated with the proportions of arachidonic acid (AA; 20:4n-6) and dihomo- $\gamma$-linolenic acid $(20: 3 n-6)$ in plasma triacylglycerols and choline phosphoglycerides.6,7 In some studies, positive associations between birth weight and docosahexaenoic acid (DHA; 22:6n-3) were described as well.7-9 $\mathrm{On}$ the Basis of these findings, a smaller size at birth seems to be related to a lower EFA status.

Besides individual EFA concentrations in plasma, erythrocytes, and tissue phospholipids, there are other indicators of biochemical EFA status. When the availability of EFAs does not meet functional requirements, the human body produces more fatty acids of comparable chain length and degree of unsaturation, such as eicosatrienoic acid (20:3n-9). Therefore, higher concentrations of eicosatrienoic acid indicate a lower EFA status. Similarly, docosapaentanoic acid $(22: 5 n-6)$ is produced when DHA availability is marginal, and the ratio between DHA and docosapaentanoic acid can be used as an indicator of DHA status. 10 A more general marker of EFA status is the ratio between the sum of all $n-3$ and $n-6$ fatty acids and the sum of all non-EFAs from the $\mathrm{n}-7$ and $\mathrm{n}-9$ families. 11 Crawford et al.5 reported "grossly abnormal" concentrations of two of these indexes in the umbilical artery walls of low-birth-weight neonates (birth weight $<2500 \mathrm{~g}$ ) which suggests again that a small size at birth is associated with a lower EFA status.

Conclusions drawn from observations in premature infants or low-birth-weight neonates might, however, not be applicable to the more common situation of term birth. Few comparable studies in healthy term neonates have been conducted. In the present study we determined both EFA concentrations and EFA-status indexes in umbilical cord plasma phospholipid samples obtained from term neonates. These indexes of biochemical EFA status were used to investigate relations with infant birth weight. In addition, we studied relations between neonatal weight and observed changes in maternal plasma EFA composition during pregnancy.

\section{Subjects and Methods}

\section{Study population}

As part of other investigations, pregnant women were asked to participate in longitudinal observational studies investigating of changes in EFA status during pregnancy and the relation of these changes to pregnancy outcome.12-15 Three antenatal 
clinics located in the province of Limburg in the southern part of the Netherlands participated: the University Hospital in Maastricht, Hospital "De Wever" in Heerlen, and the School for Midwifery in Kerkrade. Selection criteria for inclusion in these studies were a gestational age of $\leq 16 \mathrm{wk}$ at entry, a diastolic blood pressure $<90$ $\mathrm{mmHg}$, and no signs of cardiovascular, neurologic, renal, or metabolic disorders at the time of recruitment. In the present analysis, the available data for 752 singletons born between January 1990 and January 1994 during these observational studies were used. After exclusion of infants with unknown gestational age or birth weight $(n=6)$, who were born prematurely (gestational age $<37 \mathrm{wk}, n=43$ ), or who died $(n=2)$ and of mothers with diabetes $(n=14)$ or pregnancy-induced hypertension $(n=71)$, a total study population of 627 infants was left for analysis. Approval for these studies was obtained from the Ethics Committee of the University Hospital Maastricht, and all participating women gave their written, informed consent.

\section{Gestational age and birth weight}

Local hospital staff members recorded individual maternal and infant characteristics on a standardized data sheet. Additional information was obtained from medical records or by using questionnaires. Gestational age at birth (in wk) was calculated from the recorded date of delivery and the self-reported first day of the last menstrual period; fractions were expressed in decimals. If the last menstrual period was unknown, gestational age was based on early ultrasound measurements. Infants were categorized into 5 weight-for-gestational-age categories. Infants with a birth weight $\leq 10^{\text {th }}$ percentile were classified as small for gestational age (SGA) and those with a birth weight $\geq 90^{\text {th }}$ percentile as large for gestational age (LGA). Because most infants were classified as appropriate for gestational age (AGA), this category was divided into 3 subcategories: l) a birth weight $>10^{\text {th }}$ percentile but $\leq 25^{\text {th }}$ percentile, 2) a birth weight $>25^{\text {th }}$ but $<$ $75^{\text {th }}$ percentile, and 3 ) a birth weight $\geq 75^{\text {th }}$ but $<90^{\text {th }}$ percentile. This classification was based on the percentiles given by the Dutch reference standard (appropriate for length of gestation, infant sex, and birth order). 16 In addition, recorded birth weights were converted into SD scores: 17

$\mathrm{SD}$ score $=($ birth weight $\mathrm{observed}-$ mean birth weight $\mathrm{reference}) / \mathrm{SD}_{\text {reference }}$

In this way, a continuous measure for birth weight-for-gestational-age was created. A SD score of -2 corresponds to the $2.3^{\text {rd }}$ percentile and a SD score of 2 corresponds to the $97.7^{\text {th }}$ percentile of birth weight-for-gestational-age, respectively.

\section{Blood collection and determination of fatty acid composition}

Maternal venous blood samples were collected in EDTA-treated evacuated tubes at study entry $(\leq 16 \mathrm{wk}$, mean $( \pm \mathrm{SD})$ gestational age at entry was $11 \pm 3 \mathrm{wk})$ and after delivery. 
Table 3.1: Fatty acid composition of umbilical cord plasma phospholipids of term neonates. ${ }^{1}$

\begin{tabular}{|c|c|c|c|c|}
\hline & $\begin{array}{c}\text { Boys } \\
(n=347)\end{array}$ & $\begin{array}{c}\text { Girls } \\
(n=280)\end{array}$ & $\begin{array}{c}\text { All } \\
(n=627)\end{array}$ & Range \\
\hline Total fatty acids $(\mathrm{mg} / \mathrm{L}$ ) & $571.5 \pm 6.07 a$ & $613.9 \pm 7.40$ & $590.4 \pm 4.79$ & $251.5-1218.5$ \\
\hline \multicolumn{5}{|l|}{ Fatty acids (\%wt/wt) } \\
\hline $18: 2 n-6$ & $7.54 \pm 0.07$ & $7.41 \pm 0.07$ & $7.48 \pm 0.05$ & $4.30-11.98$ \\
\hline $20: 3 n-6$ & $5.06 \pm 0.05$ & $5.18 \pm 0.05$ & $5.11 \pm 0.03$ & $2.55-8.04$ \\
\hline $20: 4 n-6$ & $16.74 \pm 0.08$ & $16.89 \pm 0.09$ & $16.81 \pm 0.06$ & $11.04-21.30$ \\
\hline $22: 4 n-6$ & $0.81 \pm 0.01$ & $0.79 \pm 0.01$ & $0.80 \pm 0.01$ & $0.47-1.38$ \\
\hline $22: 5 n-6$ & $0.86 \pm 0.02$ & $0.83 \pm 0.02$ & $0.85 \pm 0.01$ & $0.25-1.93$ \\
\hline$\sum n-6$ & $32.08 \pm 0.09$ & $32.17 \pm 0.10$ & $32.12 \pm 0.07$ & $24.11-36.51$ \\
\hline$\sum \mathrm{n}-6 \mathrm{LCPs}$ & $23.46 \pm 0.08$ & $23.68 \pm 0.08$ & $23.56 \pm 0.06$ & $18.38-27.79$ \\
\hline $18: 3 n-3$ & ND & ND & ND & ND \\
\hline $20: 5 n-3$ & $0.23 \pm 0.01$ & $0.23 \pm 0.01$ & $0.23 \pm 0.00$ & $0.00-1.07$ \\
\hline $22: 5 n-3$ & $0.47 \pm 0.01$ & $0.47 \pm 0.01$ & $0.47 \pm 0.01$ & $0.14-1.21$ \\
\hline $22: 6 n-3$ & $6.20 \pm 0.07$ & $6.21 \pm 0.08$ & $6.21 \pm 0.05$ & $3.12-10.48$ \\
\hline$\sum n-3$ & $7.04 \pm 0.08$ & $7.04 \pm 0.09$ & $7.04 \pm 0.06$ & $3.57-11.69$ \\
\hline$\sum \mathrm{n}-3 \mathrm{LCPs}$ & $6.98 \pm 0.08$ & $6.70 \pm 0.09$ & $6.99 \pm 0.06$ & $3.54-11.69$ \\
\hline $20: 3 n-9$ & $0.49 \pm 0.02$ & $0.44 \pm 0.02$ & $0.47 \pm 0.01$ & $0.00-2.32$ \\
\hline$\sum n-7+n-9$ & $12.44 \pm 0.10$ & $12.28 \pm 0.12$ & $12.37 \pm 0.08$ & $6.09-24.45$ \\
\hline ¿SFAs & $47.60 \pm 0.08$ & $47.69 \pm 0.08$ & $47.64 \pm 0.06$ & $39.33-57.27$ \\
\hline ¿MUFAs & $11.89 \pm 0.09$ & $11.77 \pm 0.10$ & $11.83 \pm 0.07$ & $5.75-23.53$ \\
\hline ¿PUFAs & $39.52 \pm 0.09$ & $39.59 \pm 0.10$ & $39.55 \pm 0.07$ & $31.47-43.73$ \\
\hline
\end{tabular}

IValues are mean \pm SEM. $\sum n-6$ and $\sum n-3$, the sum of all $n-6$ and $n-3$ fatty acids, respectively; $\sum n-6$ LCPs, the sum of all $n-6$ long-chain polyenes $(20: 3 n-6,20: 4 n-6,22: 4 n-6$ and $22: 5 n-6) ; \sum n-3$ LCPs, the sum of all $n-3$ long-chain polyenes $(20: 4 n-3,20: 5 n-3,22: 5 n-3$ and $22: 6 n-3) ; \sum n-7+n-9$, the

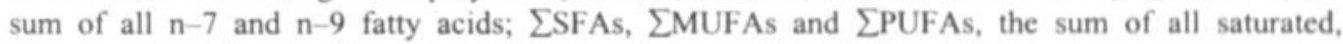
monounsaturated, and polyunsaturated fatty acids, respectively; ND, not detected.

aSignificantly different from girls, $P<0.0001$ (unpaired Student's $t$ test).

Directly after parturition, a blood sample was obtained from the umbilical vein. Plasma was separated from blood cells by centrifugation $\left(2000 \times \mathrm{g}, 4{ }^{\circ} \mathrm{C}, 15 \mathrm{~min}\right)$ and stored under nitrogen at $-80^{\circ} \mathrm{C}$ until analyzed. 18 The fatty acid composition of maternal and umbilical cord plasma phospholipids was determined as described previously.12 In short, after the addition of 1,2-dinonadecanoyl phosphatidylcholine (internal standard), total lipid extracts from $100 \mu \mathrm{L}$ plasma were prepared by using a modified 19 version of Folch et al.'s ${ }^{20}$ extraction method. Phospholipids were isolated by solid-phase extraction of total lipid extracts on aminopropyl-silica columns. ${ }^{21}$ To check for carryover of other lipid fractions during this procedure, heptadecaenoic acid (17:1) was added to the samples. After saponification of the isolated phospholipids, fatty acids were converted to the corresponding fatty acid methyl esters. 22 The fatty acid methyl esters were analyzed by capillary gas-liquid chromatography with use of a 50-m CP-Sil 5 CB non-polar capillary column (Chrompack, Middelburg, The Netherlands). Plasma total phospholipid fatty acids were expressed in absolute concentrations $(\mathrm{mg} / \mathrm{L})$ and the individual fractions of fatty acids and fatty acid groups as relative values ( $\%$ by wt of total fatty acids). 
8 Table 3.2: Characteristics of the study population according to weight-for-gestational-age percentile category at birth. ${ }^{1,2}$

\begin{tabular}{|c|c|c|c|c|c|}
\hline & \multirow[b]{2}{*}{$\begin{array}{c}\mathrm{SGA}, \leq 10^{\text {th }} \\
(n=81)\end{array}$} & \multicolumn{3}{|c|}{ AGA } & \multirow[b]{2}{*}{$\begin{array}{c}\text { LGA, } \geq 90^{\text {th }} \\
(n=41)\end{array}$} \\
\hline & & $\begin{array}{c}>10^{\text {th }} \text { to } \leq 25^{\text {th }} \\
(n=95)\end{array}$ & $\begin{array}{c}>25^{\star} \text { to }<75^{\text {क }} \\
(n=339)\end{array}$ & $\begin{array}{c}\geq 75^{\text {th }} \text { to }<90^{\mathrm{d}} \\
(n=71)\end{array}$ & \\
\hline \multicolumn{6}{|l|}{ Maternal characteristics } \\
\hline Age (y) & $28.9 \pm 4.1$ & $28.9 \pm 4.6$ & $29.5 \pm 4.2$ & $29.3 \pm 4.2$ & $29.4 \pm 3.9$ \\
\hline Height $(\mathrm{cm})$ & $163.7 \pm 6.9$ & $164.4 \pm 5.9$ & $166.6 \pm 6.6$ & $168.5 \pm 6.0$ & $170.8 \pm 6.0$ \\
\hline Weight at study entry $(\mathrm{kg})$ & $61.7 \pm 11.4$ & $61.3 \pm 9.6$ & $65.2 \pm 11.0$ & $71.4 \pm 15.8$ & $71.3 \pm 12.0$ \\
\hline Weight increase during pregnancy $(\mathrm{kg})$ & $9.7 \pm 3.8$ & $10.7 \pm 3.7$ & $11.8 \pm 3.8$ & $12.4 \pm 4.4$ & $12.5 \pm 3.9$ \\
\hline \multicolumn{6}{|l|}{ Parity $(\%)$} \\
\hline 0 & 67 & 71 & 74 & 75 & 68 \\
\hline 1 & 30 & 23 & 21 & 21 & 27 \\
\hline 2 & 1 & 5 & 4 & 4 & 5 \\
\hline 3 or more & 2 & 1 & 1 & 0 & 0 \\
\hline Smoking (\%) & 44 & 28 & 27 & 18 & 17 \\
\hline \multicolumn{6}{|l|}{ Mode of delivery $(\%)$} \\
\hline Vaginal & 77 & 79 & 77 & 79 & 68 \\
\hline Extraction (vacuum/forceps) & 11 & 12 & 17 & 13 & 17 \\
\hline Caesarian section & 12 & 9 & 6 & 8 & 15 \\
\hline \multicolumn{6}{|l|}{ Infant characteristics } \\
\hline $\operatorname{Sex}(M: F)$ & $36: 45$ & $49: 46$ & $204: 135$ & $38: 33$ & $20: 21$ \\
\hline Gestational age (wk) & $40.1 \pm 1.3$ & $40.0 \pm 1.0$ & $40.1 \pm 1.2$ & $40.6 \pm 1.2$ & $40.4 \pm 1.3$ \\
\hline Birth weight $(\mathrm{g})$ & $2661 \pm 255$ & $3006 \pm 178$ & $3380 \pm 247$ & $3856 \pm 222$ & $4166 \pm 227$ \\
\hline Weight SD score & $-1.62 \pm 0.39$ & $-0.87 \pm 0.17$ & $-0.05 \pm 0.36$ & $0.90 \pm 0.19$ & $1.63 \pm 0.32$ \\
\hline Crown-heel length $(\mathrm{cm})$ & $47.6 \pm 2.0$ & $48.8 \pm 1.7$ & $50.3 \pm 1.7$ & $51.8 \pm 1.6$ & $52.5 \pm 1.4$ \\
\hline Occipital-frontal circumference $(\mathrm{cm})$ & $33.2 \pm 1.3$ & $34.0 \pm 1.2$ & $34.4 \pm 1.3$ & $35.5 \pm 1.2$ & $36.1 \pm 1.2$ \\
\hline Apgar score after $5 \mathrm{~min}$ & $9.3 \pm 1.2$ & $9.7 \pm 0.8$ & $9.6 \pm 0.8$ & $9.7 \pm 0.6$ & $9.6 \pm 0.6$ \\
\hline Apgar score $\leq 7(\%)$ & 9 & 2 & 2 & 1 & 3 \\
\hline
\end{tabular}

'SGA, small for gestational age; AGA, appropriate for gestational age; LGA, large for gestational age. The percentile categories are based on Dutch reference standards (appropriate for length of gestation, infant sex and birth order). ${ }^{16}$

${ }^{2}$ Mean $\pm \mathrm{SD} ; n=627$. 
Table 3.3: Fatty acid composition of umbilical cord plasma phospholipids in term infants according to gestational age at birth.'

\begin{tabular}{|c|c|c|c|c|c|c|c|c|}
\hline & \multirow[b]{2}{*}{$\begin{array}{c}37 \mathrm{wk} \\
(n=26)\end{array}$} & \multirow[b]{2}{*}{$\begin{array}{c}38 \mathrm{wk} \\
(n=69)\end{array}$} & \multirow[b]{2}{*}{$\begin{array}{c}39 \text { wk } \\
(n=162)\end{array}$} & \multirow[b]{2}{*}{$\begin{array}{c}40 \mathrm{wk} \\
(n=189)\end{array}$} & \multirow[b]{2}{*}{$\begin{array}{c}41 \text { wk } \\
(n=143)\end{array}$} & \multirow[b]{2}{*}{$\begin{array}{c}42 \text { wk } \\
(n=38)\end{array}$} & \multicolumn{2}{|c|}{$P$ for trend ${ }^{2}$} \\
\hline & & & & & & & $\begin{array}{c}\text { crude } \\
(n=627)\end{array}$ & $\begin{array}{c}\text { adjusted } \\
(n=614)\end{array}$ \\
\hline Total fatty acids ( $\mathrm{mg} / \mathrm{L}$ ) & $594.6 \pm 22.5$ & $590.8 \pm 16.2$ & $580.2 \pm 10.0$ & $582.8 \pm 7.79$ & $613.6 \pm 10.1$ & $581.9 \pm 18.7$ & 0.3335 & 0.3233 \\
\hline \multicolumn{9}{|l|}{ Fatty acids (\% wt/wt) } \\
\hline $18: 2 n-6$ & $8.35 \pm 0.21$ & $7.79 \pm 0.13$ & $7.53 \pm 0.09$ & $7.38 \pm 0.08$ & $7.39 \pm 0.10$ & $6.96 \pm 0.21$ & $<0.0001$ & $<0.0001$ \\
\hline $20: 3 n-6$ & $5.04 \pm 0.18$ & $5.33 \pm 0.12$ & $5.24 \pm 0.06$ & $5.03 \pm 0.06$ & $5.00 \pm 0.07$ & $5.01 \pm 0.14$ & 0.0105 & 0.0039 \\
\hline $20: 4 n-6$ & $17.04 \pm 0.30$ & $16.61 \pm 0.21$ & $16.72 \pm 0.12$ & $16.93 \pm 0.11$ & $16.69 \pm 0.13$ & $17.21 \pm 0.25$ & 0.4008 & 0.3204 \\
\hline $22: 4 n-6$ & $0.72 \pm 0.02$ & $0.73 \pm 0.01$ & $0.80 \pm 0.01$ & $0.81 \pm 0.01$ & $0.82 \pm 0.01$ & $0.84 \pm 0.02$ & $<0.0001$ & $<0.0001$ \\
\hline $22: 5 n-6$ & $0.78 \pm 0.05$ & $0.82 \pm 0.03$ & $0.86 \pm 0.02$ & $0.85 \pm 0.02$ & $0.85 \pm 0.02$ & $0.87 \pm 0.05$ & 0.3751 & 0.2357 \\
\hline$\sum n-6$ & $33.09 \pm 0.24$ & $32.38 \pm 0.18$ & $32.26 \pm 0.12$ & $32.06 \pm 0.13$ & $31.83 \pm 0.14$ & $31.88 \pm 0.27$ & $<0.0001$ & 0.0003 \\
\hline$\sum n-6$ LCPs & $23.57 \pm 0.28$ & $23.49 \pm 0.18$ & $23.62 \pm 0.11$ & $23.62 \pm 0.11$ & $23.35 \pm 0.12$ & $23.92 \pm 0.21$ & 0.9713 & 0.9291 \\
\hline $18: 3 n-3$ & ND & ND & ND & ND & ND & ND & & \\
\hline $20: 5 n-3$ & $0.19 \pm 0.02$ & $0.22 \pm 0.01$ & $0.23 \pm 0.01$ & $0.22 \pm 0.01$ & $0.24 \pm 0.01$ & $0.24 \pm 0.02$ & 0.0303 & 0.0423 \\
\hline $22: 5 n-3$ & $0.34 \pm 0.03$ & $0.38 \pm 0.01$ & $0.45 \pm 0.01$ & $0.48 \pm 0.01$ & $0.52 \pm 0.01$ & $0.57 \pm 0.03$ & $<0.0001$ & $<0.0001$ \\
\hline $22: 6 n-3$ & $5.12 \pm 0.27$ & $5.48 \pm 0.13$ & $6.07 \pm 0.09$ & $6.25 \pm 0.09$ & $6.61 \pm 0.12$ & $7.08 \pm 0.19$ & $<0.0001$ & $<0.0001$ \\
\hline$\sum n-3$ & $5.74 \pm 0.29$ & $6.19 \pm 0.14$ & $6.90 \pm 0.11$ & $7.08 \pm 0.11$ & $7.51 \pm 0.14$ & $8.06 \pm 0.22$ & $<0.0001$ & $<0.0001$ \\
\hline$\sum n-3$ LCPs & $5.72 \pm 0.29$ & $6.15 \pm 0.14$ & $6.86 \pm 0.11$ & $7.04 \pm 0.11$ & $7.46 \pm 0.14$ & $7.98 \pm 0.22$ & $<0.0001$ & $<0.0001$ \\
\hline $20: 3 n-9$ & $0.44 \pm 0.06$ & $0.53 \pm 0.03$ & $0.47 \pm 0.02$ & $0.47 \pm 0.02$ & $0.43 \pm 0.02$ & $0.41 \pm 0.03$ & 0.0299 & 0.0060 \\
\hline$\sum n-7+n-9$ & $12.90 \pm 0.36$ & $13.22 \pm 0.22$ & $12.33 \pm 0.11$ & $12.27 \pm 0.13$ & $12.22 \pm 0.21$ & $11.68 \pm 0.20$ & $<0.0001$ & $<0.0001$ \\
\hline$\sum$ SFAs & $47.55 \pm 0.18$ & $47.31 \pm 0.17$ & $47.72 \pm 0.09$ & $47.78 \pm 0.11$ & $47.57 \pm 0.14$ & $47.44 \pm 0.20$ & 0.4809 & 0.6155 \\
\hline ¿MUFAs & $12.37 \pm 0.30$ & $12.61 \pm 0.20$ & $11.78 \pm 0.09$ & $11.73 \pm 0.12$ & $11.73 \pm 0.20$ & $11.21 \pm 0.17$ & $<0.0001$ & $<0.0001$ \\
\hline ¿PUFAs & $39.18 \pm 0.34$ & $39.02 \pm 0.19$ & $39.54 \pm 0.12$ & $39.54 \pm 0.13$ & $39.70 \pm 0.16$ & $40.28 \pm 0.24$ & 0.0003 & 0.0006 \\
\hline
\end{tabular}

Values are unadjusted means \pm SEM. For legend see also Table $3.1 ; \mathrm{ND}$, not detected.

${ }^{2} P$ values for linear trends with the continuous variable gestational age are given crude and adjusted for potential confounding factors (maternal age, weight at entry, weight increase during pregnancy, smoking, parity, mode of delivery, 5-min Apgar score, infant sex and weight SD score). 
Table 3.4: Fatty acid composition of umbilical cord plasma phospholipids in term infants according to weight-for-gestational-age percentile category at birth.'

\begin{tabular}{|c|c|c|c|c|c|c|c|}
\hline & \multirow[b]{2}{*}{$\begin{array}{c}\text { SGA, } \leq 10^{\text {in }} \\
(n=81)\end{array}$} & \multicolumn{3}{|c|}{ AGA } & \multirow[b]{2}{*}{$\begin{array}{l}\mathrm{LGA}, \geq 90^{\text {由 }} \\
\qquad(n=41)\end{array}$} & \multicolumn{2}{|c|}{$P$ for trend ${ }^{2}$} \\
\hline & & $\begin{array}{l}>10^{\text {th }} \text { to } \leq 25^{\text {th }} \\
(n=95)\end{array}$ & $\begin{array}{c}>25^{\text {th }} \text { to }<75^{\text {th }} \\
(n=339)\end{array}$ & $\begin{array}{c}275^{\text {th }} \text { to }<90^{\text {th }} \\
(n=71)\end{array}$ & & $\begin{array}{c}\text { crude } \\
(n=627)\end{array}$ & $\begin{array}{l}\text { adjusted } \\
(n=614)\end{array}$ \\
\hline Total fatty acids (mg/L) & $573.9 \pm 12.6$ & $576.7 \pm 13.3$ & $588.7 \pm 6.48$ & $621.2 \pm 12.5$ & $615.9 \pm 20.2$ & 0.0006 & $<0.0001$ \\
\hline \multicolumn{8}{|l|}{ Fatty acids (\%wt/wt) } \\
\hline $18: 2 n-6$ & $7.60 \pm 0.13$ & $7.51 \pm 0.12$ & $7.48 \pm 0.06$ & $7.35 \pm 0.14$ & $7.42 \pm 0.19$ & 0.0426 & 0.2131 \\
\hline $20: 3 n-6$ & $4.73 \pm 0.10$ & $5.03 \pm 0.09$ & $5.18 \pm 0.05$ & $5.18 \pm 0.09$ & $5.35 \pm 0.11$ & $<0.0001$ & $<0.0001$ \\
\hline $20: 4 n-6$ & $17.61 \pm 0.18$ & $17.01 \pm 0.14$ & $16.68 \pm 0.09$ & $16.60 \pm 0.18$ & $16.23 \pm 0.22$ & $<0.0001$ & $<0.0001$ \\
\hline $22: 4 n-6$ & $0.80 \pm 0.02$ & $0.82 \pm 0.02$ & $0.79 \pm 0.01$ & $0.78 \pm 0.02$ & $0.79 \pm 0.02$ & 0.1925 & 0.0574 \\
\hline $22: 5 n-6$ & $0.78 \pm 0.03$ & $0.85 \pm 0.03$ & $0.85 \pm 0.02$ & $0.86 \pm 0.03$ & $0.95 \pm 0.05$ & 0.0006 & 0.0001 \\
\hline$\sum n-6$ & $32.57 \pm 0.17$ & $32.32 \pm 0.17$ & $32.06 \pm 0.09$ & $31.80 \pm 0.19$ & $31.81 \pm 0.23$ & 0.0007 & 0.0021 \\
\hline$\sum n-6 L C P s$ & $23.92 \pm 0.16$ & $23.71 \pm 0.14$ & $23.49 \pm 0.08$ & $23.42 \pm 0.18$ & $23.31 \pm 0.21$ & 0.0371 & 0.0166 \\
\hline $18: 3 n-3$ & ND & ND & ND & ND & ND & & \\
\hline $20: 5 n-3$ & $0.23 \pm 0.01$ & $0.22 \pm 0.01$ & $0.23 \pm 0.01$ & $0.24 \pm 0.02$ & $0.22 \pm 0.01$ & 0.9161 & 0.5722 \\
\hline $22: 5 n-3$ & $0.51 \pm 0.02$ & $0.47 \pm 0.02$ & $0.46 \pm 0.01$ & $0.48 \pm 0.02$ & $0.45 \pm 0.02$ & 0.0373 & 0.0003 \\
\hline $22: 6 n-3$ & $6.56 \pm 0.16$ & $6.28 \pm 0.13$ & $6.13 \pm 0.07$ & $6.32 \pm 0.17$ & $5.74 \pm 0.19$ & 0.0072 & $<0.0001$ \\
\hline$\sum n-3$ & $7.42 \pm 0.17$ & $7.11 \pm 0.14$ & $6.96 \pm 0.08$ & $7.17 \pm 0.19$ & $6.52 \pm 0.21$ & 0.0108 & $<0.0001$ \\
\hline$\sum n-3$ LCPs & $7.38 \pm 0.17$ & $7.06 \pm 0.14$ & $6.91 \pm 0.08$ & $7.13 \pm 0.19$ & $6.49 \pm 0.21$ & 0.0112 & $<0.0001$ \\
\hline $20: 3 n-9$ & $0.35 \pm 0.03$ & $0.43 \pm 0.03$ & $0.48 \pm 0.02$ & $0.49 \pm 0.03$ & $0.61 \pm 0.05$ & $<0.0001$ & $<0.0001$ \\
\hline$\sum n-7+n-9$ & $11.67 \pm 0.20$ & $12.00 \pm 0.16$ & $12.49 \pm 0.11$ & $12.59 \pm 0.21$ & $13.16 \pm 0.28$ & $<0.0001$ & $<0.0001$ \\
\hline ¿SFAs & $47.42 \pm 0.15$ & $47.73 \pm 0.17$ & $47.66 \pm 0.08$ & $47.57 \pm 0.15$ & $47.77 \pm 0.14$ & 0.3284 & 0.4106 \\
\hline$\Sigma$ MUFAs & $11.26 \pm 0.19$ & $11.50 \pm 0.15$ & $11.94 \pm 0.10$ & $12.02 \pm 0.18$ & $12.49 \pm 0.23$ & $<0.0001$ & $<0.0001$ \\
\hline ¿PUFAs & $40.27 \pm 0.16$ & $39.79 \pm 0.18$ & $39.43 \pm 0.09$ & $39.38 \pm 0.22$ & $38.86 \pm 0.23$ & $<0.0001$ & $<0.0001$ \\
\hline
\end{tabular}

Values are unadjusted means \pm SEM. For legend see also Table 3.1 and Table 3.2. ND, not detected.

${ }^{2} P$ values for linear trends with the continuous variable weight SD score are given crude and adjusted for potential confounding factors (maternal age, weight at entry, weight increase during pregnancy, smoking, parity, mode of delivery, 5-min Apgar score, infant sex and pregnancy duration). 
In total, 39 fatty acids were identified but, for clarity, only the concentrations of 9 individual fatty acids and 8 fatty acid groups are reported (Table 3.1). In addition to the reported concentrations, two indexes of EFA status were calculated: the DHA-status index (ratio of DHA to docosapentaenoic acid (22:5n-6); a higher ratio indicates a higher DHA status) 10 and the EFA-status index (ratio of $\sum n-3+n-6$ to $\sum n-7+n-9$; a higher ratio indicates a higher EFA status). 11

\section{Statistical analysis}

Values are reported as mean \pm SEMs, unless specified otherwise. Differences between means were evaluated by either paired or unpaired two-tailed Student's $t$ tests. Relations between variables were analyzed with simple and multiple regression models. To test for linear trends, the continuous variable weight SD score was used instead of the weight-for-gestational-age categories. Maternal age, maternal weight at study entry, weight increase during pregnancy, smoking habits, parity (ordinal), mode of delivery (dummies for extraction and caesarian section with vaginal delivery as a reference) and the 5-min Apgar score were included as potential confounding factors. When the total study sample was analyzed, infant sex was introduced as an additional factor. Because of a skewed distribution of some variables (20:5n-3, 20:3n-9 and DHA-status index) $\log$ or square-root transformed data were used in the analyses. Because of incomplete data records, not all analyses were based on the same number of subjects. A two-tailed $P$ value $<0.05$ was considered statistically significant. All statistical analyses were performed by using STATVIEW (version 4.5; Abacus Concepts Inc., Berkeley, CA).

\section{Results}

Characteristics of the women and their neonates are listed in Table 3.2. Birth weight ranged from 1875 to $4350 \mathrm{~g}$ in girls and from 2050 to $4620 \mathrm{~g}$ in boys. In total, 81 (13 $\%)$ infants were born SGA according to Dutch references. 16 Forty-one (7\%) neonates were born LGA. On average, birth weight deviated from the reference mean by -0.16 SDs (range in weight SD score: -3.23 to 2.57 ). Weight SD scores did not differ significantly between boys and girls (mean \pm SD: $-0.14 \pm 0.87$ and $-0.18 \pm 0.92$, respectively) by unpaired Student's $t$ test.

\section{Infant sex and the EFA composition of plasma phospholipids}

The total amount $(\mathrm{mg} / \mathrm{L})$ of fatty acids in umbilical cord plasma phospholipids of female neonates was larger than that observed in males (Table 3.1). This difference, however, was not due to a higher concentration of one particular fatty acid or fatty acid group. The proportions of each individual fatty acid and that of specific fatty acid groups did not differ between boys and girls. In addition, the total amount of fatty acids and the relative fatty acid composition of maternal plasma phospholipids did not differ between boys and girls, nor was there any sex-related difference in the observed 
changes in relative fatty acid concentrations in maternal plasma phospholipids during pregnancy. For these reasons, further comparisons were done with boys and girls combined. Infant sex was used as an additional factor in the multiple regression models, but no major effect of sex on fatty acid fractions or relations were found.

The fatty acid $\alpha$-linolenic acid (18:3n-3) was present in such low concentrations in umbilical plasma phospholipids that it could not be detected in most of the samples. In only $34 \%$ of the infants $\alpha$-linolenic acid was detected in measurable amounts. The median $\alpha$-linolenic acid concentration in these subjects was $0.12 \%$ by wt of total fatty acids [inter quartile range (IQR): $0.07 \%$ by wt of total fatty acids]. In the total group of infants, median $\alpha$-linolenic acid concentration was $0.00 \%$ by wt of total fatty acids (IQR: $0.08 \%$ by wt of total fatty acids). The number of infants in whom $\alpha$-linolenic acid could be measured did not differ significantly between boys and girls, gestational age groups, or weight-for-gestational-age groups (chi-square tests).

\section{Gestational age and the fatty acid composition of umbilical plasma phospholipids}

Infants born after a shorter duration of pregnancy had relatively higher linoleic acid and higher $\sum \mathrm{n}-6$ fatty acid concentrations in their umbilical cord plasma phospholipids (Table 3.3). In contrast, $20: 4 n-6$ and $\sum n-6$ long chain polyene (LCP) concentrations were not significantly related to gestational age at birth. Most pronounced, however, were the differences in the $\mathrm{n}-3$ fatty acid fractions. Neonates born at a later gestational age had higher umbilical cord plasma concentrations of eicosapentaenoic acid (EPA; $20: 5 n-3)$, docosapentaenoic acid (22:5n-3), DHA, $\sum n-3$ and $\sum n-3$ LCPs, whereas the proportions of eicosatrienoic acid (20:3n-9) and $\sum n-7+n-9$ fatty acids were lower in these infants. The total fatty acid content was not related to gestational age at birth.

\section{Birth weight and the fatty acid composition of umbilical cord plasma phospholipids}

The relative EFA composition of umbilical cord plasma phospholipids was associated with weight-for-gestational-age at birth (Table 3.4). Fractions of AA, $\sum \mathrm{n}-6, \sum \mathrm{n}-6$ LCPs and $\sum$ PUFAs were lower in infants born LGA. Both dihomo- $\gamma$-linolenic acid (20:3n-6) and docosapentaenoic acid (22:5n-6) concentrations were, however, higher in heavier neonates. Furthermore, docosapentaenoic acid (22:5n-3), DHA, $\sum \mathrm{n}-3$ and $\sum \mathrm{n}-3$ LCP concentrations were higher in the smaller infants, whereas the proportion of EPA was not related to weight-for-gestational-age at birth. In addition to these observations, $20: 3 n-9, \sum n-7+n-9$ fatty acids and the sum of monounsaturated fatty acids ( $\sum$ MUFAs) concentrations were evidently higher in umbilical cord plasma samples of heavier infants. The total amount of plasma phospholipid fatty acids was also higher in heavier infants.

\section{Gestational age, birth weight and indicators of EFA status}

The EFA-status index was higher in the umbilical cord plasma of neonates born at a later time point but was lower in those born relatively large for gestational age (Figure 
3.1, A \& C). Similarly, the DHA-status index was higher in plasma of infants born after a longer duration of gestation and lower in those with a higher weight-for-gestationalage at birth (Figure 3.1, B \& D). In Table 3.5, the association between weight-forgestational-age at birth and the EFA-status index is compared with the relations of key prognostic indicators of fetal growth and infant size at birth.

\section{EFA composition of maternal plasma phospholipids}

Of all 627 selected neonates, information on the EFA composition of 582 maternal plasma samples taken at study entry ( $\leq 16 \mathrm{wk}$ gestation) and of 568 maternal plasma samples taken at delivery were available. In a total of 546 cases $(87 \%)$, the fatty acid compositions of both samples were known.

The well-known differences in relative fatty acid composition between maternal and umbilical cord plasma were observed (Tables $3.2 \&$ 3.6). The concentration of $\alpha$ linolenic acid was significantly higher in plasma phospholipids of mothers of infants in whom $\alpha$-linolenic acid concentrations were measurable than in those of mothers of infants with undetectable amounts of $\alpha$-linolenic acid $(0.27 \pm 0.10 \%$ compared with $0.19 \pm 0.10 \%$ by wt of total fatty acids; $P<0.0001$ ).

During pregnancy, the total fatty acid concentration increased and its composition changed significantly (Table 3.6). At delivery, relatively more saturated fatty acids and MUFAs were found, whereas the fraction of PUFAs was lower. The EFA-status index and the DHA-status index also decreased during pregnancy (Table 3.6). The observed changes in the mother's plasma EFA compositions were related to the size of their infants (Figures $3.2 \&$ \& 3.3). The biggest decrease in plasma concentrations of AA, DHA, $\sum \mathrm{n}-6$ LCP and $\sum \mathrm{n}-3$ LCPs were observed in mothers of heavier infants, whereas the largest reduction in the fraction of linoleic acid was found in the mothers of relatively smaller neonates.

No cross-sectional association was found between maternal fatty acid concentrations and infant size at birth at study entry or at delivery. There also was no relation between maternal plasma fatty acid concentrations and the total duration of gestation. The fatty acid concentrations in maternal plasma were strong predictors of umbilical cord plasma fatty acid composition. However, the reported relations between umbilical cord plasma fatty acid composition and normalized birth weight were independent of the observed maternal concentrations.

\section{Discussion}

To our knowledge, this was the first study to show relations between umbilical cord plasma phospholipid EFA composition and size at birth in term neonates. Both the pattern of individual fatty acid fractions and the EFA-status indexes seemed to indicate a higher biochemical EFA status in umbilical cord plasma of smaller infants than in the plasma of larger ones. 
EFA-status index

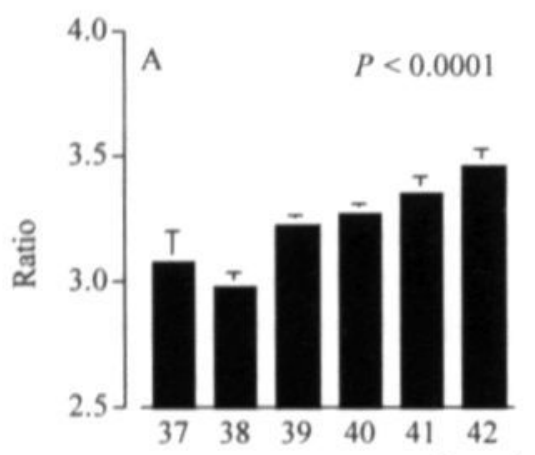

DHA-status index

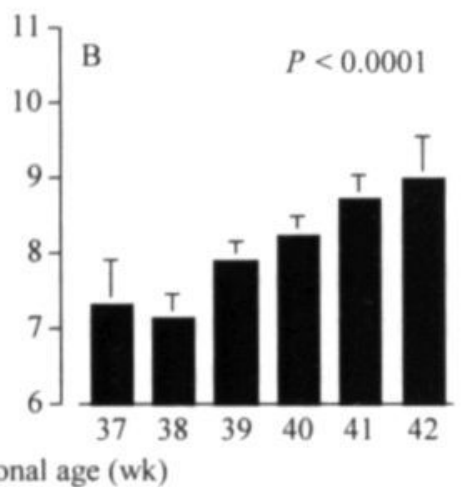

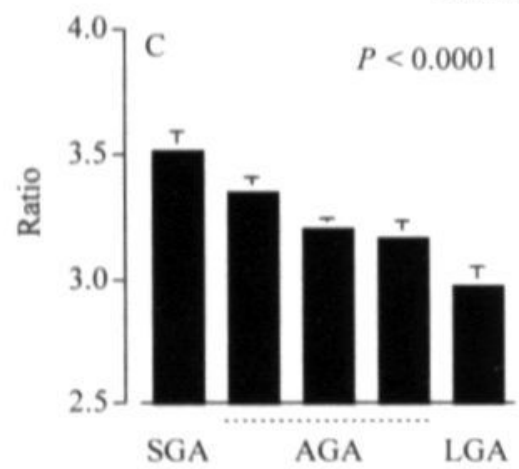

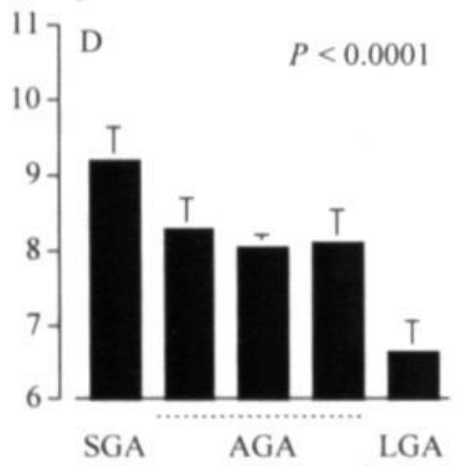

Weight-for-gestational-age category

Figure 3.1: Mean ( \pm SEM) essential fatty acid (EFA)-status index (ratio of $\sum n-3+n-6$ to $\sum n-7+n-9$ ) and docosahexaenoic acid (DHA)-status index (ratio of 22:6n-3 to 22:5n-6) in umbilical cord plasma phospholipids of infants according to gestational age at birth (A \& B) and infant birth weight, i.e., weight-for-gestational-age category (C \& D). The categories are small for gestational age (SGA; birth weight $\leq 10^{\text {th }}$ percentile), appropriate for gestational age (AGA; divided into 3 subcategories: $I$ ) birth weight $>10^{\text {th }}$ but $\leq 25^{\text {th }}$ percentile, 2 ) $>25^{\text {th }}$ but $<75^{\text {th }}$ percentile, and 3 ) $\geq 75^{\text {th }}$ but $<90^{\text {th }}$ percentile), and large for gestational age (LGA; birth weight $\geq 90^{\text {th }}$ percentile). All percentiles were based on Dutch reference standards (appropriate for length of gestation, infant sex, and birth order). ${ }^{16}$ The $P$ values shown are for linear trends with the continuous variable gestational age or weight SD score. 
Table 3.5: Association of weight-for-gestational-age at birth with the essential fatty acid-status index measured in umbilical cord plasma phospholipids and prognostic indicators of fetal growth. 1

\begin{tabular}{|c|c|c|c|}
\hline & $\beta$ & SE & $t$ \\
\hline \multicolumn{4}{|l|}{ Univariate analysis } \\
\hline EFA-status index & $-0.343 \mathrm{a}$ & 0.060 & -5.8 \\
\hline Maternal weight at study entry $(\mathrm{kg})$ & 0.019 a & 0.003 & 6.5 \\
\hline Weight increase during pregnancy $(\mathrm{kg})$ & 0.049 a & 0.009 & 5.6 \\
\hline Maternal height $(\mathrm{cm})$ & 0.041 a & 0.005 & 7.7 \\
\hline Maternal smoking $($ yes $=1 ;$ no $=0$ ) & -0.353 a & 0.079 & -4.5 \\
\hline Maternal age $(y)$ & 0.010 & 0.008 & 1.2 \\
\hline \multicolumn{4}{|l|}{ Multivariate analysis } \\
\hline EFA-status index & -0.369 a & 0.055 & -6.7 \\
\hline Maternal weight at study entry $(\mathrm{kg})$ & $0.018 \mathrm{a}$ & 0.003 & 6.3 \\
\hline Weight increase during pregnancy $(\mathrm{kg})$ & 0.051 a & 0.008 & 6.3 \\
\hline Maternal height $(\mathrm{cm})$ & 0.024 a & 0.005 & 4.7 \\
\hline Maternal smoking $($ yes $=1 ;$ no $=0$ ) & $-0.266 \mathrm{~b}$ & 0.072 & -3.7 \\
\hline Maternal age (y) & 0.004 & 0.008 & 0.5 \\
\hline
\end{tabular}

Essential fatty acid (EFA)-status index, ratio of $\sum n-3+n-6$ to $\sum n-7+n-9$.

IResults of simple and multiple regression analyses with weight SD score (appropriate for length of gestation, infant sex, and birth order) ${ }^{16}$ as the dependent variable and the EFA-status index and prognostic indicators of fetal growth as independent variables.

a $P<0.0001 ; \mathrm{b} P<0.001$.

\section{EFAs as determinants of fetal growth}

The concept that EFAs such as AA or DHA may serve as potential fetal growth factors was not supported by our results. Proportions of AA and DHA in umbilical cord plasma phospholipids were negatively related to neonatal size at birth (Table 3.4). In addition, no relation was found between the concentrations of these fatty acids in maternal plasma and infant birth weight. These findings are in contrast with previous observations in premature infants and low-birth-weight babies.5-7,23 In most of these studies, lower proportions of AA, DHA, or both were found in smaller neonates. An explanation for this inconsistency between studies could be that additional (pathologic) factors associated with premature birth or severe growth retardation affected both EFA concentrations and intrauterine growth in these populations. Another factor that might play a role is gestational age at birth. Both birth weight and the biochemical EFA status of newborns are related to the duration of pregnancy (see also Chapter 2).24,25 No adjustment for differences in gestational age at birth might therefore have confounded some of the previously reported associations. When birth weights were interpreted in relation to gestation duration in comparisons of SGA with AGA or LGA infants, no differences in plasma or vessel wall AA concentrations where found by several investigators.7,8,26,27 In one study, the reported relative DHA concentration was even significantly higher in SGA babies.? However, most of these findings were based on observations in relatively small numbers of infants. 
Table 3.6: Fatty acid composition of maternal plasma phospholipids. ${ }^{1}$

\begin{tabular}{|c|c|c|c|c|}
\hline is & $\begin{array}{l}\text { Study entry } \\
(n=582)\end{array}$ & $\begin{array}{l}\text { Delivery } \\
(n=568)\end{array}$ & $\begin{array}{l}\text { Chan } \\
(n=5\end{array}$ & $\begin{array}{l}\operatorname{lge}^{2, \text { a }} \\
546)\end{array}$ \\
\hline Total fatty acids (mg/L) & $1329.5 \pm 10.5$ & $1759.4 \pm 13.0$ & $433.0 \pm 14.6$ & $(404.2 ; 461.7)$ \\
\hline \multicolumn{5}{|l|}{ Fatty acids (\% wt $/ w t)$} \\
\hline $18: 2 n-6$ & $21.48 \pm 0.11$ & $20.72 \pm 0.10$ & $-0.76 \pm 0.10$ & $(-0.96 ;-0.57)$ \\
\hline $20: 3 n-6$ & $3.08 \pm 0.03$ & $3.46 \pm 0.03$ & $0.37 \pm 0.03$ & $(0.32 ; 0.42)$ \\
\hline $20: 4 n-6$ & $9.61 \pm 0.06$ & $8.55 \pm 0.06$ & $-1.07 \pm 0.05$ & $(-1.17 ;-0.97)$ \\
\hline $22: 4 n-6$ & $0.39 \pm 0.00$ & $0.38 \pm 0.00$ & $-0.02 \pm 0.00$ & $(-0.02 ;-0.01)$ \\
\hline $22: 5 n-6$ & $0.35 \pm 0.01$ & $0.53 \pm 0.01$ & $0.18 \pm 0.01$ & $(0.17 ; 0.19)$ \\
\hline$\sum n-6$ & $35.55 \pm 0.08$ & $34.33 \pm 0.08$ & $-1.24 \pm 0.08$ & $(-1.40 ;-1.08)$ \\
\hline$\sum n-6 L C P s$ & $13.44 \pm 0.07$ & $12.92 \pm 0.07$ & $-0.53 \pm 0.06$ & $(-0.64 ;-0.42)$ \\
\hline $18: 3 n-3$ & $0.21 \pm 0.01$ & $0.22 \pm 0.00$ & $0.01 \pm 0.01$ & $(-0.01 ; 0.02)$ \\
\hline $20: 5 n-3$ & $0.55 \pm 0.02$ & $0.35 \pm 0.01$ & $-0.20 \pm 0.02$ & $(-0.23 ;-0.17)$ \\
\hline $22: 5 n-3$ & $0.75 \pm 0.01$ & $0.55 \pm 0.01$ & $-0.20 \pm 0.01$ & $(-0.22 ;-0.19)$ \\
\hline $22: 6 n-3$ & $4.04 \pm 0.04$ & $3.87 \pm 0.03$ & $-0.18 \pm 0.03$ & $(-0.24 ;-0.11)$ \\
\hline$\sum n-3$ & $5.69 \pm 0.05$ & $4.93 \pm 0.04$ & $-0.77 \pm 0.04$ & $(-0.85 ;-0.68)$ \\
\hline$\sum \mathrm{n}-3 \mathrm{LCPs}$ & $5.48 \pm 0.05$ & $4.91 \pm 0.04$ & $-0.58 \pm 0.04$ & $(-0.66 ;-0.49)$ \\
\hline $20: 3 n-9$ & $0.19 \pm 0.01$ & $0.25 \pm 0.01$ & $0.06 \pm 0.00$ & $(0.05 ; 0.06)$ \\
\hline$\sum n-7+n-9$ & $11.95 \pm 0.05$ & $13.08 \pm 0.06$ & $1.13 \pm 0.06$ & $(1.02 ; 1.25)$ \\
\hline ¿SFAs & $44.36 \pm 0.05$ & $45.60 \pm 0.04$ & $1.24 \pm 0.05$ & $(1.14 ; 1.33)$ \\
\hline ¿MUFAs & $11.72 \pm 0.05$ & $12.80 \pm 0.06$ & $1.08 \pm 0.06$ & $(0.97 ; 1.19)$ \\
\hline ¿PUFAs & $41.44 \pm 0.06$ & $39.63 \pm 0.06$ & $-1.83 \pm 0.07$ & $(-1.96 ;-1.67)$ \\
\hline DHA-status index & $12.85 \pm 0.25$ & $8.21 \pm 0.24$ & $-4.71 \pm 0.21$ & $(-5.11 ;-4.30)$ \\
\hline EFA-status index & $3.50 \pm 0.02$ & $3.05 \pm 0.02$ & $-0.45 \pm 0.02$ & $(-0.50 ;-0.41)$ \\
\hline
\end{tabular}

IValues are mean \pm SEM $(95 \% \mathrm{CI})$. For legend see also Table 3.1. DHA-status index, ratio of $22: 6 \mathrm{n}-3$ to $22: 5 n-6$; EFA-status index, ratio of $\sum n-3+n-6$ to $\sum n-7+n-9$.

2 Difference between fatty acid concentrations at delivery and at study entry ( $\leq 16 \mathrm{wk}$ ).

aAll differences were significant at $P<0.0001$ (paired Student's $t$ test), except for $\alpha$-linolenic acid $(18: 3 n-3)$.

AA and DHA were also shown to be related to postnatal growth. During the first year after birth, negative effects of fish-oil-supplemented formula (rich in DHA and EPA) on infant growth were described in premature infants. $28 \mathrm{~A}$ reduction in the AA status was regarded as a causative factor in the observed growth restriction. ${ }^{29}$ However, some intervention studies in term neonates found no such effect of DHA supplementation (with or without AA) on postnatal growth. ${ }^{30-33}$ To our knowledge, no studies of the effects of formulas supplemented with AA alone on infant growth have been conducted. A potential effect of AA and DHA on neonatal growth, therefore, is still controversial.

In contrast with $\mathrm{AA}$ and DHA concentrations, concentrations of dihomo- $\gamma$ linolenic acid $(20: 3 n-6)$ were positively related to normalized birth weight (Table 3.4). Lower concentrations of dihomo- $\gamma$-linolenic acid in the blood or vessel walls of smaller than of larger neonates were reported previously and were also found in premature infants. $6-8,26,27,34$ The positive association between dihomo- $\gamma$-linolenic acid concentration and size at birth seems to be more consistent than that reported for AA or 
DHA. Therefore, dihomo- $\gamma$-linolenic acid may be more important for intrauterine growth than AA. No study has yet evaluated the effect of dihomo- $\gamma$-linolenic acid supplementation on intrauterine or postnatal growth.

\section{Maternal-to-fetal EFA supply}

The $\mathrm{n}-3$ and $\mathrm{n}-6$ long-chain PUFAs (especially AA and DHA) are important structural and functional components of cell membranes. Therefore, a larger infant probably accretes more of these substances than does a smaller one. Because the fetal capacity to convert linoleic acid and $\alpha$-linolenic acid into LCPs is limited, ${ }^{35-38}$ most of these LCPs are obtained from the maternal circulation via the placenta. The observation that umbilical cord plasma EFA concentrations are positively associated with both maternal plasma EFA concentrations and maternal dietary EFA intake 14 supports this notion.

In a subgroup of the participating women, previously published information on the dietary intake of fatty acids 14,15 was available (based on food frequency questionnaires and dietary history). The most important finding was a relatively high intake of linoleic acid ( $\pm 6 \%$ of total energy intake and $\pm 85 \%$ of total PUFA intake). Such a high linoleic acid intake might explain the low $\alpha$-linolenic acid concentrations found in umbilical cord plasma. Indeed, the dietary ratio of linoleic acid to other PUFAs (mainly $\alpha$-linolenic acid) was significantly lower in mothers of infants in whom $\alpha$ linolenic acid could be detected than in mothers of infants with undetectable amounts of $\alpha$-linolenic acid $(P<0.05)$. Moreover, higher maternal plasma $\alpha$-linolenic acid concentrations were found in mothers of infants with detectable $\alpha$-linolenic acid concentrations. The maternal intake of fatty acids, however, did not differ between the weight-for-gestational-age groups (data not shown). The current finding that decreases in maternal plasma n-3 LCP and n-6 LCP fractions are more pronounced in women who gave birth to larger infants (Figure $3.2 \& 3.3$ ) implies that the EFA transfer from the mother to the fetus is related to fetal growth. It is possible that larger infants are born when the maternal-to-fetal transfer of LCPs is more efficient. However, an increased LCP transfer could also be an adaptation to an increased fetal LCP accretion. Because umbilical cord plasma EFA concentrations are positively related to maternal plasma EFA concentrations, whereas birth weights of infants are not, the latter explanation seems more likely.

The observed lower relative concentrations of AA and DHA, higher concentrations of plasma 20:3n-9 (Table 3.4), and lower EFA-status and DHA-status indexes (Figure 3.1) in the plasma of heavier neonates suggest that the maternal-to-fetal supply of EFAs is limited. It seems that even an increased maternal-to-fetal LCP flux cannot prevent a lower biochemical EFA status in plasma of heavier neonates. We showed previously that the biochemical EFA status determined on the basis of EFA concentrations in the cord plasma and vessel walls of twins and triplets is lower than observed in singletons. 39,40 

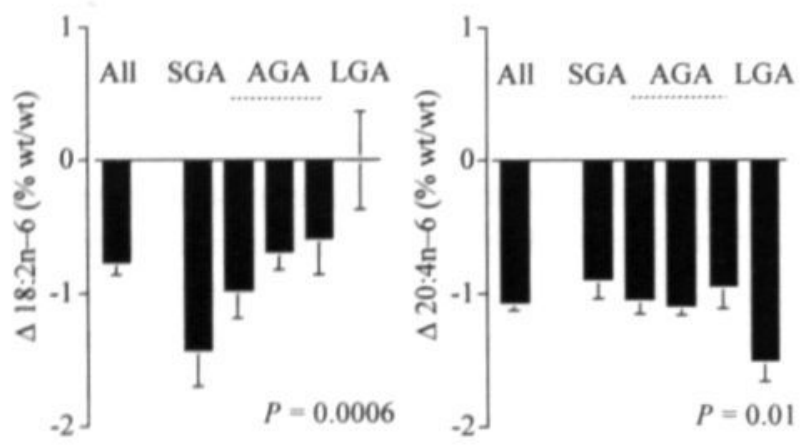

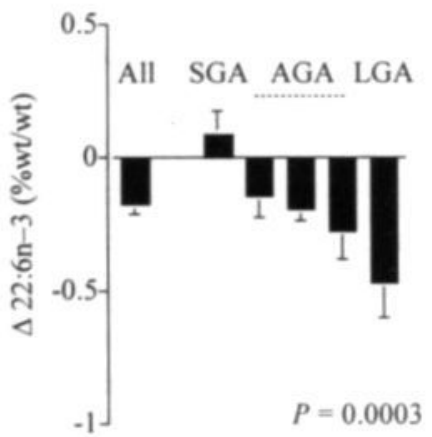

Figure 3.2: Mean ( \pm SEM) changes in linoleic acid $(18: 2 n-6)$, arachidonic acid $(A A ; 20: 4 n-6)$ and docosahexaenoic acid (DHA; 22:6n-3) concentration in maternal plasma phospholipids during pregnancy for the total study group and according to infant birth weight, i.e., weight-for-gestationalage category. The categories are small for gestational age (SGA; birth weight $\leq 10^{\text {th }}$ percentile), upproprnite $G_{1}$ gestativinat age (AUA; arviaed into 5 suocategories: 1 ) birth weight $>10^{\text {th }}$ but $\leq 25^{\text {th }}$ percentile, 2) $>25^{\text {th }}$ but $<75^{\text {th }}$ percentile, and 3 ) $\geq 75^{\text {th }}$ but $<90^{\text {th }}$ percentile), and large for gestational age (LGA; birth weight $\geq 90^{\text {th }}$ percentile). All percentiles were based on Dutch reference standards (appropriate for length of gestation, infant sex, and birth order). ${ }^{16}$ The $P$ values shown are for linear trends with the continuous variable weight SD score.

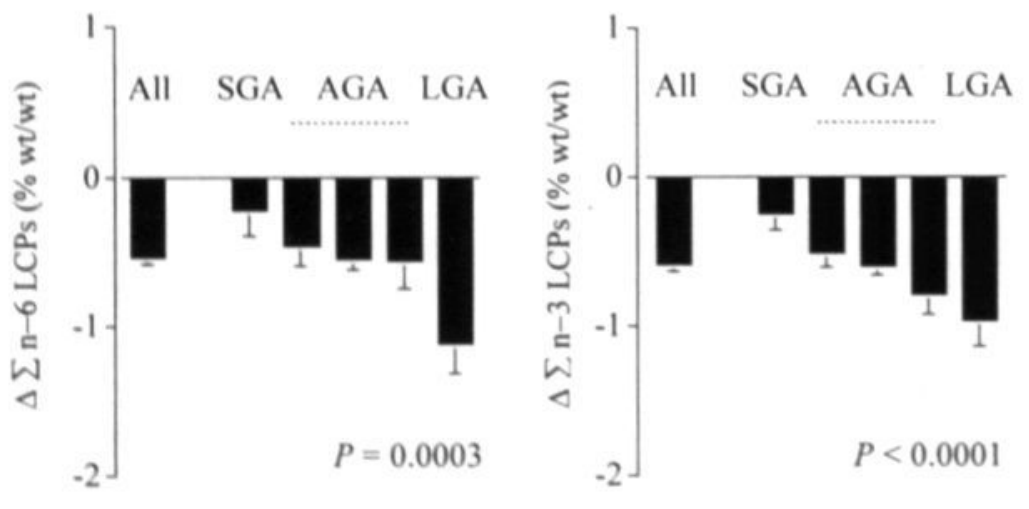

Figure 3.3: Mean ( \pm SEM) changes in $n-6$ long-chain polyenes ( $\sum n-6$ LCPs) and $n-3$ long-chain polyenes $\left(\sum \mathrm{n}-3 \mathrm{LCPs}\right)$ in maternal plasma phospholipids during pregnancy for the total study group and according to birth weight, i.e. weight-for-gestational-age category. The $P$ values shown are for linear trends with the continuous variable weight SD score. For legend see also Figure 3.2. 
These observations seem to support the concept that a larger total fetal tissue mass is related to an increased EFA accretion and the idea that the supply of EFA is limited. However, these hypotheses are based on observed associations and further evidence is needed to validate them. Studies using more advanced techniques, such as stable isotopes, are needed to evaluate complex dynamic processes like LCP accretion and placental transport efficiency.

\section{Changes in linoleic acid concentration}

The pregnancy-associated decrease in linoleic acid concentration was more pronounced in mothers of smaller infants than in mothers of larger infants (Figure 3.2). The reason for this is not clear. Relative linoleic acid concentrations tend to remain stable until the end of pregnancy and the decrease shown in Figure 3.2 occurs mainly around the time of delivery. ${ }^{12}$ In contrast, the observed decreases in the fractions of AA and DHA start before 16 and 22 wk of gestation, respectively.12 Thus, it seems unlikely that these opposite patterns of observed decreases are directly related, e.g. because of competitive or selective placental transfer of fatty acids.

\section{Nutritional sufficiency of EFA status in plasma of term neonates}

Although low EFA concentrations did not seem to be associated with limited growth in the present study, the specific neonatal demand for EFAs may not have been met. ${ }^{41,42}$ We are presently conducting a long-term follow-up study on the children born during our studies to investigate potential functional consequences of early EFA status in later life. Without such information, statements about the nutritional sufficiency of the EFA status of term infants, on the basis of EFA concentrations found in umbilical cord plasma, remain speculative.

In summary, under the present dietary conditions, EFAs such as AA and DHA do not seem to be important determinants of fetal growth in term neonates. The biochemical EFA status measured in umbilical cord plasma of term neonates is even negatively associated with size at birth. This lower EFA status in plasma of heavier infants occurred despite a larger decrease in LCP fractions in maternal plasma. These findings suggest that the maternal-to-fetal EFA transfer capacity is a limiting factor in determining neonatal EFA status. However, the implications of these findings for mother and children are not known and remain to be investigated.

\section{References}

1. Burr GO, Burr MM. A new deficiency disease produced by rigid exclusion of fat from the diet. J Biol Chem. 1929;82:345-367.

2. Burr GO, Burr MM. On the nature and role of the fatty acids essential in nutrition. $J$ Biol Chem. 1930;86:587-621. 


\section{Chapter 3}

3. Hansen AE, Wiese HF, Boelsche AN, Haggard ME, Adam DJD, Davis H. Role of linoleic acid in infant nutrition. Pediatrics. 1963;31:171-192.

4. Caldwell MD, Jonsson HT, Othersen HB. Essential fatty acid deficiency in an infant receiving prolonged parental alimentation. J Pediatr. 1972;81:894-898.

5. Crawford MA, Doyle W, Drury P, Lennon A, Costeloe K, Leighfield M. n-3 and n-6 fatty acids during early human development. J Intern Med. 1989;225:159-169.

6. Koletzko B, Braun M. Arachidonic acid and early human growth: is there a relation? Ann Nutr Metab. 1991;35:128-131.

7. Leaf AA, Leighfield MJ, Costeloe KL, Crawford MA. Long chain polyunsaturated fatty acids and fetal growth. Early Hum Dev. 1992;30:183-191.

8. Felton CV, Chang TC, Crook D, Marsh M, Robson SC, Spencer JAD. Umbilical vessel wall fatty acids after normal and retarded fetal growth. Arch Dis Child. 1994;70:F36-F39.

9. Foreman-van Drongelen MMHP, Houwelingen ACv, Kester ADM, Hasaart THM, Blanco CE, Hornstra G. Long-chain polyunsaturated fatty acids in pre-term infants: status at birth and its influence on postnatal levels. J Pediatr. 1995;126:611-618.

10. Hoffman DR, Uauy R. Essentiality of dietary omega-3 fatty acids for premature infants; plasma and red blood cell fatty acid composition. Lipids. 1992;27:886-895.

11. Hornstra G, Al MDM, Houwelingen ACv, Foreman-van Drongelen MMHP. Essential fatty acids in pregnancy and early human development. Eur J Obstet Gynecol Reprod Biol. 1995;61:57-62.

12. Al MD, Houwelingen $\mathrm{ACv}$, Kester AD, Hasaart TH, de Jong AE, Hornstra G. Maternal essential fatty acid patterns during normal pregnancy and their relationship to the neonatal essential fatty acid status. Br J Nutr. 1995;74:55-68.

13. Al MD, Houwelingen ACv, Badart-Smook A, Hasaart TH, Roumen FJ, Hornstra G. The essential fatty acid status of mother and child in progmancy-inutuceu' hypertension: a prospective longitudinal study. Am J Obstet Gynecol. 1995;172:1605-14.

14. Al MD, Badart-Smook A, Houwelingen ACv, Hasaart TH, Hornstra G. Fat intake of women during normal pregnancy: relationship with maternal and neonatal essential fatty acid status. $J$ Am Coll Nutr. 1996;15:49-55.

15. Badart-Smook A, Houwelingen ACv, Al MDM, Kester ADM, Hornstra G. Fetal growth is associated positively with maternal intake of riboflavin and negatively with maternal intake of linoleic acid. J Am Diet Assoc. 1997;97:867-870.

16. Kloosterman GJ. On intrauterine growth: The significance of prenatal care. Int J Gyneacol Obstet. 1970;8:895-912.

17. World Health Organization. Physical status: the use and interpretation of anthropometry. Report of a WHO expert commitee. World Health Organ Tech Rep Ser. 1995;854.

18. Otto SJ, Foreman-van Drongelen MMHP, Houwelingen ACv, Hornstra G. Effects of storage on venous and capillary blood samples: the influence of deferoxamine and butylated hydroxytoluene on the fatty acid alterations in red blood cell phospholipids. Eur J Clin Chem Clin Biochem. 1997;35:907-913.

19. Hoving EB, Jansen G, Volmer M, Doormaal JJv, Muskiet FAJ. Profiling of plasma triglyceride fatty acids as their methylesters by capillary gas chromatography, preceded by a rapid aminopropyl silica column chromatographic seperation of lipid classes. J Chromatogr. 1988:434:395-409.

20. Folch J, Lees M, Sloane-Stanley GH. A simple method for the isolation and purification of total lipids from animal tissues. J Biol Chem. 1957;226:497-509.

21. Kaluzny MA, Duncan LA, Merritt MV, Epps DE. Rapid separation of lipid classes in high yield and purity using bonded phase columns. $J$ Lipid Res. 1985;26:135-140.

22. Morrisson WR. Smith LM. Preparation of fatty acid methyl esters and demethylacatals from lipids with boron fluoride methanol. J Lipid Res. 1964;5:600-8. 
23. Woltil HA, Beusekom CMv, Schaafsma A, Muskiet FAJ, Okken A. Long-chain polyunsaturated fatty acid status and early growth of low birth weight infants. Eur $J$ Pediatr. 1998; 157:146-152.

24. Hoving ED, Beusekom CMv, Nijboer HJ, Muskiet FAJ. Gestational age dependency of essential fatty acids in cord plasma cholesterol esters and triglycerides. Pediatr Res. 1994:35:461-469.

25. Houwelingen ACv, Foreman-van Drongelen MMHP, Nicolini U, Nicolaides KH, Al MDM, Kester ADM, Hornstra G. Essential fatty acid status of fetal plasma phospholipids: similar to postnatal values obtained at comparable gestational ages. Early Hum Dev. 1996;46:141-152.

26. Vilbergsson G, Samsioe G, Wennergren M, Karlsson K. Essential fatty acids in pregnancies complicated by intrauterine growth retardation. Int J Gyneacol Obstet. 1991;36:277-286.

27. Percy P, Vilbergsson G, Percy A, Mansson J, Wennergren M, Svennerholm L. The fatty acid composition of placenta in intrauterine growth retardation. Biochim Biophys Acta. 1991;1084:173-177.

28. Carlson SE, Cooke RJ, Werkman SH, Tolley EA. First year growth of preterm infants fed standard compared to marine oil supplemented formula. Lipids. 1992;27:901-907.

29. Carlson SE, Werkman SH, Peeples JM, Cooke RJ, Tolley EA. Arachidonic acid status correlates with first year growth in preterm infants. Proc Natl Acad Sci USA. 1993;90:10731077.

30. Auested N, Montalto MB, Hall RT, Fitzgerald KM, Wheeler RE, Conner WE, Neuringer M, Conner SL, Taylor JA, Hartmann EE. Visual acuity, erythrocyte fatty acid composition, and growth in term infants fed formulas with long chain polyunsaturated fatty acids for one year. Pediatr Res. 1997;41:1-10.

31. Birch EE, Hoffman DR, Uauy R, Birch DG, Prestidge C. Visual acuity and the essentiality of docosahexaenoic acid and arachidonic acid in the diet of term infants. Pediatr Res. 1998;44:201-209.

32. Makrides M, Neumann MA, Simmer K, Gibson RA. Dietary long-chain polyunsaturated fatty acids do not influence growth of term infants: a randomized clinical trial. Pediatrics. 1999; 104:468-475.

33. Lucas A, Stafford M, Morley R, Abbott R, Stephenson T, MacFadeyen U, Elias-Jones A, Clements $\mathrm{H}$. Efficacy and safety of long-chain polyunsaturated fatty acid supplementation of infant-formula milk : a randomised trail. Lancet. 1999;354:1948-54.

34. Farquharson J, Jamieson EC, Logan RW, McFadyn MB, Patrick WJA, Howatson AG, Cockburn F. Infant growth and aorta total lipid fatty acids. Arch Dis Child. 1998;79:28-32.

35. Salem N, Wegher B, Mena P, Uauy R. Arachidonic and docosahexaenoic acids are biosynthesized from their 18-carbon precursors in human infants. Proc Natl Acad Sci USA. 1996;93:49-54.

36. Koletzko B, Decsi T, Demmelmair H. Arachidonic acid supply and metabolism in human infants born at full term. Lipids. 1996;31:79-83.

37. Rodriguez A, Sarda P, Nessmann C, Boulot P, Poisson JP, Leger CL, Descomps B. Fatty acid desaturase activities and polyunsaturated fatty acid composition in human liver between the seventeeth and thirty-sixth gestational weeks. Am J Obstet Gynecol. 1998;179:1063-1070.

38. Hamosh M, Salem N. Long-chain polyunsaturated fatty acids. Biol Neonate. 1998;74:106-120.

39. Foreman-van Drongelen MMHP, Zeijdner EE, Houwelingen ACv, Kester ADM, Al MDM, Hasaart TH, Hornstra G. Essential fatty acid status measured in umbilical vessel walls of infants born after multiple pregnancy. Early Hum Dev. 1996;46:205-215.

40. Zeijdner EE, Houwelingen ACv, Kester ADM, Hornstra G. Essential fatty acid status in plasma phospholipids of mother and neonate after multiple pregnancy. Prostaglandins Leukotr Essent Fatty Acids. 1997;56:395-401. 


\section{Chapter 3}

41. Crawford MA. The role of essential fatty acids in neural development implications for perinatal nutrition. Am J Clin Nutr. 1993;57:703S-710S.

42. Sattar N, Berry C, Greer IA. Essential fatty acids in relation to pregnancy complications and fetal development. Br J Obstet Gynaecol. 1998;105:1248-1255. 
Chapter 4 Components of the insulin resistance syndrome in seven-year-old children: relations with birth weight and the polyunsaturated fatty acid content of umbilical cord plasma phospholipids

P. Rump, C. Popp-Snijders, R.J. Heine, and G. Hornstra

Diabetologia 2002:45:349-355 


\begin{abstract}
Aims/hypothesis According to the fetal origins hypothesis, there is a relation between fetal nutrition and adult glucose intolerance. In adults, insulin resistance has been associated with dietary polyunsaturated fatty acids. We examined whether the availability of polyunsaturated fatty acids during fetal life, as indicated by the fatty acid composition of cord blood samples, relates to childhood body composition and glycaemic control.
\end{abstract}

Methods Fatty acid concentrations in umbilical cord blood were determined by gasliquid chromatography in a birth-cohort of infants. When the children were seven years old, fasting glucose, insulin, proinsulin, and leptin levels were measured in 259 of these children, and relations with cord plasma fatty acid concentrations were studied.

Results Cord plasma phospholipid $\gamma$-linolenic acid and dihomo- $\gamma$-linolenic acid concentrations were negatively related to insulin concentration and calculated insulin resistance (homeostasis model assessment) at seven years of age. The $\gamma$-linolenic acid concentration was also negatively related to body fatness and proinsulin and leptin concentrations at seven years of age. No association was found with other polyunsaturated fatty acid concentrations at birth. Adjusted for age, sex, current weight, and gestational age, a lower birth weight related to higher values of the insulin resistance variables. The highest insulin concentrations were found in children with a low birth weight and a low $\gamma$-linolenic acid concentration at birth. The relations between $\gamma$-linolenic acid concentration at birth and fasting insulin and calculated insulin resistance remained statistically significant when adjusted for birth weight.

Conclusion/interpretation These findings indicate that fetal availability or metabolism of $\gamma$-linolenic acid could be involved in the early origins of insulin resistance. 


\section{Introduction}

Insulin resistance is an important component of the metabolic syndrome that encompasses a cluster of risk factors for cardiovascular diseases including Type II (non-insulin-dependent) diabetes mellitus, dyslipedaemia, obesity, and hypertension. ${ }^{1,2}$ A small size at birth has been associated with an increased risk for the development of insulin resistance and Type II diabetes mellitus later in life. ${ }^{3-8}$ According to the fetal origins hypothesis, which was based on these epidemiological findings, the propensity to develop insulin resistance might be a consequence of structural and functional adaptations to a limited availability of nutrients during fetal life. ${ }^{9}$

Birth weight is, however, only a crude indicator of fetal nutrition and other environmental or genetic factors could explain the associations between size at birth and adult Type II diabetes mellitus. Still, there is some evidence that fetal nutrition could indeed be involved, ${ }^{10}$ like the epidemiological findings of the Dutch Hunger Winter study ${ }^{11}$ and results from experiments in pregnant animals. ${ }^{12-14}$ The availability or balance of particular nutrients rather than the availability of macronutrients could be important. ${ }^{10}$ Thus far, only a limited number of human studies have been conducted on the nutritional factors that could play a role. One such group of nutrients that could be important in the fetal origins of adult diseases are essential fatty acids and their polyunsaturated derivatives. ${ }^{15,16}$ The importance of the fatty acid composition of our diet for the development of insulin resistance has been recognized for some years now. ${ }^{17-19}$ Several studies have shown that the fatty acid composition of serum lipid fractions and muscle membrane phospholipids - which at least partly reflect dietary fat quality - are closely related to insulin action. ${ }^{20-23}$ For instance, the development of Type II diabetes has been related to lower concentrations of linoleic acid $(18: 2 n-6)$ and higher concentrations of palmitoleic acid (16:1n-7), $\gamma$-linolenic acid $(18: 3 n-6)$, and dihomo- $\gamma$-linolenic acid $(20: 3 n-6)$ in serum cholesterol esters of 50-year-old men. ${ }^{24}$ Previous comparisons between breast-fed and bottle-fed subjects seem to indicate that nutritional factors early in life, like the availability of polyunsaturated fatty acids (PUFAs), could indeed play a role in the development of adult Type II diabetes mellitus. $^{25,26}$

Whether the fetal availability of PUFAs relates to the development of insulin resistance later in life is not known. The primary objective of our study was therefore to explore whether the availability of PUFAs in utero relates to fasting glucose, insulin, proinsulin and leptin concentrations later in life. For this purpose we conducted: follow-up study on a cohort of children in whom umbilical cord plasma phospholipid PUFA concentrations had been determined at birth. We used these umbilical cord plasma phospholipid PUFA concentrations as biomarkers of intrauterine PUFA availability. 
Table 4.1: Characteristics of the study population by sex.'

\begin{tabular}{llll} 
& \multicolumn{1}{c}{ Boys } & \multicolumn{1}{c}{ Girls } \\
& $(n=142)$ & $(n=117)$ \\
\hline Length of gestation (wk) & $39.8(39.1 ; 41.0)$ & $39.9(39.1 ; 41.0)$ \\
Birth weight $(\mathrm{g})$ & $3377(504)$ & $3203(499)^{\mathrm{b}}$ \\
Age $(\mathrm{y})$ & $7.3(0.3)$ & $7.3(0.3)$ \\
Weight $(\mathrm{kg})$ & $25.5(4.4)$ & $24.8(4.0)$ \\
Height $(\mathrm{cm})$ & $127.8(5.0)$ & $126.1(5.9)^{\mathrm{a}}$ \\
BMI $\left(\mathrm{kg} / \mathrm{m}^{2}\right)$ & $15.4(14.3 ; 16.4)$ & $15.4(14.3 ; 16.5)$ \\
Sum four skinfolds $(\mathrm{mm})$ & $24.1(19.9 ; 26.4)$ & $28.4(23.4 ; 33.0)^{\mathrm{c}}$ \\
Body fat $(\%)$ & $16.2(13.8 ; 18.0)$ & $20.9(18.3 ; 23.7)^{\mathrm{c}}$ \\
Fat mass $(\mathrm{kg})$ & $4.1(3.2 ; 4.8)$ & $5.1(4.0 ; 6.4)^{\mathrm{c}}$ \\
Fat-free mass $(\mathrm{kg})$ & $21.1(2.7)$ & $19.4(2.3)^{\mathrm{c}}$ \\
Leptin $(\mu \mathrm{L} / \mathrm{L})$ & $2.7(1.9 ; 3.6)$ & $3.8(2.6 ; 4.9)^{\mathrm{c}}$ \\
Glucose $(\mathrm{mmol} / \mathrm{L})$ & $4.7(0.4)$ & $4.6(0.3)^{\mathrm{a}}$ \\
Insulin (pmol/L) & $32.9(25.0 ; 44.0)$ & $36.2(27.8 ; 48.0)$ \\
Proinsulin (pmol/L) & $6.4(5.4 ; 7.5)$ & $6.9(5.6 ; 8.5)^{\mathrm{a}}$ \\
Proinsulin to insulin ratio & $0.20(0.14 ; 0.25)$ & $0.19(0.15 ; 0.25)$ \\
Insulin resistance (HOMA) & $1.0(0.7 ; 1.3)$ & $1.0(0.8 ; 1.4)$ \\
Beta-cell function (HOMA) & $78.1(60.6 ; 99.6)$ & $90.9(71.2 ; 123.9)^{\mathrm{b}}$ \\
\hline BMI, body mass
\end{tabular}

BMI, body mass index; HOMA, homeostasis model assessment.

'Values are mean (SD) except for gestational age, BMI, sum four skinfolds, percent body fat, fat mass, leptin, insulin, pro-insulin, and the HOMA indexes for insulin resistance and $\beta$-cell function, where values are geometric mean (inter-quartile range).

${ }^{\mathrm{A}} P<0.05 ;{ }^{\mathrm{b}} P<0.01 ;{ }^{\mathrm{C}} P<0.001$ for differences between sexes.

\section{Subjects and Methods}

\section{Study cohort}

Subjects were recruited from a Dutch birth-cohort of children. As infants, these children and their mothers participated in a previous study of essential fatty acids during pregnancy and pregnancy outcome. No interventions were provided. To explore the potential long-term effects of fetal essential fatty acid availability, a follow-up study was carried out between 1997 and 2000. All singleton babies born alive before 1994, and for whom an umbilical cord blood sample was available for the determination of the essential fatty acid status at birth, were included. In total, 750 children were eligible for follow-up (for more details on this cohort see Rump et al.). ${ }^{27}$ With the aid of the Dutch population registry, $97 \%$ of the eligible population could be traced. Three children had died and 34 lived abroad. The parents of the remaining 691 children received a written invitation for the follow-up evaluation. We were able to contact the parents of 558 children. Of these, 297 participated, 231 refused, and 30 retracted their initial consent. Birth characteristics (i.e. birth weight, crown-heel length, occipitalfrontal circumference, ponderal index, gestational age, birth order) of the children who participated did not differ from the children who did not attend our clinic. Approval for this study was obtained from the Ethics Committee of the University Hospital 
Maastricht and the parents of all participating children gave their written informed consent.

\section{Data collection at follow-up}

All children were investigated before puberty, at about seven years of age (range: 6.7 8.1 years). The same physician (PR) examined all children and performed all venipunctures. A questionnaire and a structured interview with one or both parents were used to collect additional information. Included were information on maternal smoking during pregnancy, infant nutrition, parental education, and family history of Type II diabetes. A family history of Type Il diabetes was considered when a parent or grandparent of the child had Type II diabetes. Anthropometric measurements were done by one observer (PR) as previously described. ${ }^{28}$ The percentage of body fat, fat mass, and fat-free mass were estimated from these skinfold measurements using equations appropriate for children. ${ }^{29}$ The use of other equations ${ }^{30}$ did not influence the main findings of this study.

\section{Blood sampling and laboratory analyses}

Not all parents and children agreed to a venipuncture, and no more than two puncture attempts were made. As a consequence, blood samples were obtained in 264 of the 297 children. The blood samples were collected in EDTA-treated evacuated tubes after an overnight fast. Plasma was separated from blood cells by centrifugation and stored in small portions at $-80{ }^{\circ} \mathrm{C}$ until analysis. The fatty acid composition of umbilical cord plasma phospholipids was determined by capillary gas-liquid chromatography, as described before. ${ }^{31}$ Glucose was determined enzymatically (Glucose HK-method, Hoffmann-La Roche, Basel, Switzerland) and specific insulin and total proinsulin were measured as described by Ruige et al. ${ }^{32}$ Plasma leptin concentration was measured using a human leptin radioimmunoassay (Linco Research, St. Charles, MO, USA). Estimates of pancreatic beta-cell function and relative insulin resistance were calculated from the fasting insulin and glucose concentrations using the homeostasis model assessment (HOMA) equations. ${ }^{33}$

\section{Statistical analysis}

All analyses were restricted to those children for whom a blood sample was obtained at follow-up. Five cases were excluded for missing values, leaving 259 children for analysis. Data are presented as mean \pm standard deviation (SD), unless specified otherwise. Differences between groups were evaluated by unpaired two-tailed Student's $t$ tests or $\chi^{2}$ (chi square) tests. Linear regression models were used to explore relations between plasma phospholipid fatty acid concentrations, birth weight and the outcome variables. During these analysis continuous variables were used instead of categories. 


\section{Chapter 4}

Table 4.2: Unadjusted mean anthropometric measurements and biochemical parameters in seven-yearold children according to tertiles of $\gamma$-linolenic acid concentration in the plasma phospholipid fraction of umbilical cord blood at birth.

\begin{tabular}{|c|c|c|c|c|c|}
\hline & \multicolumn{3}{|c|}{$\begin{array}{c}\text { Tertiles of } \gamma \text {-linolenic acid } \\
(\% w t / w t)\end{array}$} & \multicolumn{2}{|c|}{$P$ for trend ${ }^{1}$} \\
\hline & $\begin{array}{c}<0.04 \\
(n=85)\end{array}$ & $\begin{array}{c}0.04-0.07 \\
(n=87)\end{array}$ & $\begin{array}{c}\geq 0.07 \\
(n=87)\end{array}$ & unadjusted & adjusted $^{2}$ \\
\hline Weight (kg) & 25.6 & 24.9 & 25.1 & NS & - \\
\hline Height $(\mathrm{cm})$ & 127.4 & 126.7 & 127.0 & NS & NS \\
\hline BMI $\left(\mathrm{kg} / \mathrm{m}^{2}\right)$ & 15.7 & 15.4 & 15.5 & NS & NS \\
\hline Sum four skinfolds (mm) & 28.7 & 26.7 & 26.6 & 0.009 & NS \\
\hline Body fat $(\%)$ & 19.8 & 18.0 & 18.4 & 0.003 & NS \\
\hline Fat mass (kg) & 5.2 & 4.6 & 4.7 & 0.010 & NS \\
\hline Fat-free mass (kg) & 20.4 & 20.3 & 20.4 & NS & NS \\
\hline Leptin $(\mu \mathrm{g} / \mathrm{L})$ & 4.2 & 3.8 & 3.3 & 0.003 & 0.047 \\
\hline Glucose $(\mathrm{mmol} / \mathrm{L})$ & 4.7 & 4.7 & 4.6 & NS & NS \\
\hline Insulin $(\mathrm{pmol} / \mathrm{L})$ & 40.4 & 37.3 & 34.7 & 0.004 & 0.011 \\
\hline Proinsulin $(\mathrm{pmol} / \mathrm{L})$ & 7.2 & 6.9 & 6.6 & 0.025 & NS \\
\hline Proinsulin to insulin ratio & 0.20 & 0.21 & 0.22 & NS & NS \\
\hline Insulin resistance (HOMA) & 1.2 & 1.1 & 1.0 & 0.004 & 0.011 \\
\hline Beta-cell function (HOMA) & 94.8 & 91.1 & 85.2 & 0.033 & NS \\
\hline
\end{tabular}

BMI, body mass index; HOMA, homeostasis model assessment; NS, not significant.

' $P$ values are derived from simple (unadjusted) and multiple (adjusted) linear regression using continuous variables.

${ }^{2}$ Adjusted for sex, age, current weight and $\gamma$-linolenic acid concentration at follow-up.

Because of a skewed distribution of some variables, log-transformed data were used when appropriate. Multiple regression models were applied to control for potential confounding factors. A two-tailed $P$ value of less than 0.05 was considered to be statistically significant. All statistical analyses were done using STATVIEW (version 4.5, Abacus Concepts Inc., Berkeley, CA, USA).

\section{Results}

Sex, current weight and body composition

None of the children were diabetic (fasting glucose levels $<7.0 \mathrm{mmol} / \mathrm{L}$ ). The characteristics of the participating boys and girls are reported in Table 4.1. Most of the outcome variables were higher in children with a higher current weight, length, fat mass, or fat-free mass (not shown).

\section{Relations with plasma phospholipid fatty acid composition}

The most consistent relations were found between the outcome variables and the $\gamma$ linolenic acid (18:3n-6; a derivative of the essential fatty acid linoleic acid) content of the umbilical cord plasma phospholipid fraction. A higher concentration of $\gamma$-linolenic 
acid was associated with lower fasting insulin, proinsulin, HOMA insulin resistance, and HOMA beta-cell function (Table 4.2). In addition, indices of body fatness (fasting leptin, sum four skinfolds, percent body fat, and fat mass) were also negatively related to the $\gamma$-linolenic acid levels at birth, whereas, weight, height, BMI, and fat-free mass did not relate to umbilical cord $\gamma$-linolenic acid concentrations (Table 4.2).

In contrast, plasma phospholipid $\gamma$-linolenic acid concentration at seven years of age was positively related to the parameters of glycaemic control (fasting glucose, insulin, proinsulin, HOMA insulin resistance, and HOMA beta-cell function) and fasting leptin concentration $(P<0.05$ for all, unadjusted and adjusted for age, sex and current weight; data not shown). Plasma phospholipid $\gamma$-linolenic acid concentration at seven years of age did not relate to the anthropometric indices of body fatness (sum skinfolds, percent body fat, or fat mass). The associations found between $\gamma$-linolenic acid concentration at birth and fasting insulin and leptin concentrations, and HOMA insulin resistance values remained statistically significant when adjusted for age, sex, current weight and the plasma phospholipid $\gamma$-linolenic acid content at seven years of age (Table 4.2). The associations found between $\gamma$-linolenic acid concentration at birth and indices of body fatness (sum four skinfolds, percent body fat, and fat mass) were statistically significant $(P<0.05$ for all) when adjusted for sex, age and the plasma phospholipid $\gamma$-linolenic acid content at seven years of age. However, when also corrected for current weight, these relations were no longer significant (Table 4.2). No significant association or interaction was found between $\gamma$-linolenic acid concentrations at birth and $\gamma$-linolenic acid concentrations at seven years.

In addition to $\gamma$-linolenic acid, umbilical cord plasma phospholipid dihomo- $\gamma$ linolenic acid ( $20: 3 n-6$; the elongation product of $\gamma$-linolenic acid) concentrations were also negatively related with fasting insulin concentration and HOMA insulin resistance at age seven $(P<0.05$ for both, after adjustment for sex, age and current weight). Plasma phospholipid dihomo- $\gamma$-linolenic acid at seven years of age did not relate to the outcome variables. The concentration of linoleic acid (18:2n-6), arachidonic acid $(20: 4 n-6)$, eicosapentaenoic acid $(20: 5 n-3)$, docosahexaenoic acid $(22: 6 n-3)$, the sum of all $n-6$ fatty acids, the sum of all $n-6$ long-chain polyenes $(20: 3 n-6,20: 4 n-6,22: 4 n-$ 6 , and $22: 5 n-6$ ), the sum of all $n-3$ fatty acids, the sum of all $n-3$ long-chain polyenes $(20: 4 n-3,20: 5 n-3,22: 5 n-3$, and $22: 6 n-3)$ and the ratio of $n-3$ to $n-6$ fatty acids, in umbilical cord plasma phospholipids were not related any of the outcome variables. Adjustments for sex, age and current weight or the fatty acid concentrations measured at seven years of age did not change these results.

\section{Relations with birth weight}

Children of lower birth weight had a lower fat-free mass but not a lower fat mass at age seven. After adjustment for sex, age, current weight, and length of gestation, birth weight was negatively related to BMI, sum four skinfolds, percentage of body fat, fat 
mass, leptin, insulin, HOMA insulin resistance and HOMA beta-cell function (for more information see Chapter 7).

\section{Low birth weight and low $\gamma$-linolenic acid concentration at birth}

The highest fasting insulin concentration and HOMA insulin resistance values were seen in children of low birth weight who had a low $\gamma$-linolenic acid concentration at birth (Table 4.3). These children also had the lowest proinsulin to insulin ratio (Table 4.3). Additionally, children in the lowest tertile of birth weight and the lowest tertile of $\gamma$-linoleic acid concentration at birth had the highest mean fasting leptin concentration and percentage of body fat (not shown). We found no statistically significant association between birth weight and the $\gamma$-linolenic acid concentration in umbilical cord plasma phospholipids. The associations between $\gamma$-linoleic acid concentration at birth and fasting insulin concentration and HOMA insulin resistance at seven years of age remained statistically significant $(P<0.01$, for both) when adjusted for birth weight by multiple regression analysis. The product terms (birth weight times $\gamma$-linoleic acid) for interactions were not statistically significant.

\section{Family history of Type II diabetes mellitus}

Because genetic factors play a role in the origins of insulin resistance and Type II diabetes, we investigated relations with a positive family history of diabetes mellitus. Eight children were born to mothers who reported diabetes or impaired glucose tolerance during pregnancy; 78 children had a parent or grandparent with Type II diabetes. As shown in Table 4.4, a low $\gamma$-linolenic acid concentration at birth was related to a positive family history of Type II diabetes. Of the eight children born to mothers with diabetes or impaired glucose tolerance during pregnancy, seven had a below median $\gamma$-linolenic acid concentration at birth $\left(P=0.03, \chi^{2}\right.$ test $)$. Of the children with a positive family history for Type II diabetes, $51(65 \%)$ had a below median $\gamma$ linolenic acid concentration at birth $\left(P=0.005, \chi^{2}\right.$ test $)$.

The $\gamma$-linolenic acid concentration at follow-up was not related to a maternal or family history of diabetes. Excluding children with a positive family history of diabetes from the analysis did not change the main findings of our study. Fasting plasma insulin, proinsulin and leptin concentrations, HOMA insulin resistance, and percent body fat at age seven remained negatively associated with the $\gamma$-linoleic acid content of umbilical cord plasma $(P<0.05$, for all).

\section{Multiple regression analysis}

To determine how the different variables were related to fasting insulin concentrations and HOMA insulin resistance, backward step-wise multiple regression analyses were performed (Table 4.5). 
Table 4.3: Fasting insulin concentration, proinsulin to insulin ratios and insulin resistance (HOMA) values in seven-year-old children according to tertiles of umbilical cord plasma phospholipid $\gamma$ linolenic acid concentration and birth weight.

\begin{tabular}{|c|c|c|c|c|}
\hline \multirow[t]{2}{*}{ Tertiles of birth weight (g) } & \multicolumn{3}{|c|}{ Tertiles of $\gamma$-linolenic acid (\% wt/wt) } & \multirow[t]{2}{*}{$P$ for trend } \\
\hline & $<0.04$ & $0.04-0.07$ & $\geq 0.07$ & \\
\hline \multicolumn{5}{|l|}{ Insulin (pmol/L) } \\
\hline$<3100$ & $44.4(35)$ & $34.3(24)$ & $27.8(29)$ & 0.001 \\
\hline $3100-3500$ & $31.2(26)$ & $32.4(35)$ & $34.7(27)$ & NS \\
\hline$\geq 3500$ & $34.7(24)$ & $37.6(28)$ & $32.8(31)$ & NS \\
\hline$P$ for trend ${ }^{\prime}$ & 0.020 & NS & NS & $\cdots$ \\
\hline \multicolumn{5}{|l|}{ Proinsulin to insulin ratio } \\
\hline$<3100$ & 0.16 & 0.18 & 0.23 & 0.011 \\
\hline $3100-3500$ & 0.21 & 0.20 & 0.19 & NS \\
\hline$\geq 3500$ & 0.20 & 0.19 & 0.19 & NS \\
\hline$P$ for trend ${ }^{1}$ & 0.025 & NS & NS & $\cdots$ \\
\hline \multicolumn{5}{|l|}{ Insulin resistance (HOMA) } \\
\hline$<3100$ & 1.3 & 1.0 & 0.8 & 0.001 \\
\hline $3100-3500$ & 0.9 & 0.9 & 1.0 & NS \\
\hline$\geq 3500$ & 1.0 & 1.1 & 0.9 & NS \\
\hline$P$ for trend $^{1}$ & 0.013 & NS & NS & ... \\
\hline
\end{tabular}

Removed from both models $(P>0.1)$ were age and the product terms birth weight times $\gamma$-linolenic acid concentration at birth, birth weight times current weight, and family history of diabetes times $\gamma$-linolenic acid concentration at birth. There was a significant interaction between birth weight and a family history of Type II diabetes. A lower birth weight was associated higher fasting insulin concentrations and HOMA insulin resistance values only in children with a positive family history for Type II diabetes mellitus $(P<0.001$ for both). In children with a negative family history for Type II diabetes mellitus, birth weight was not related to fasting insulin concentration nor to HOMA insulin resistance ( $P=0.6$ for both). Further adjustments for maternal smoking, infant feeding mode, maternal education, paternal education, or birth order had no major influence on the outcome.

\section{Discussion}

In this study, body composition and insulin resistance variables at seven years of age 
related to birth weight and PUFA concentrations in umbilical cord blood. This data suggests that a low intrauterine availability of $\gamma$-linolenic acid could predispose individuals to an increased body fatness and insulin resistance later in life. This would be in accordance with the general concept of the fetal origin hypothesis that a limited availability of nutrients early in life could have detrimental effects for the subsequent development of insulin resistance and Type II diabetes. ${ }^{9}$

Similar to previous studies conducted in adults and children, ${ }^{3-8,34}$ we found that (after correction for sex, age, current weight and length of gestation) a small size at birth related to higher levels of the insulin resistance variables and an increased body fatness later in life. In our cohort of seven-year-olds, a low birth weight not only related to a higher HOMA index for insulin resistance, but also to higher values of HOMA beta-cell function and lower proinsulin-to-insulin ratios. These findings support an association between birth weight and insulin resistance, but do not indicate a relation with an impaired beta-cell function.

In this study, fasting insulin concentrations and the HOMA index for insulin resistance were highest in children who had a low birth weight in combination with a low umbilical cord plasma phospholipid $\gamma$-linolenic acid concentration. After adjustment for birth weight, cord blood $\gamma$-linoleic acid concentration remained associated with HOMA insulin resistance and fasting insulin levels. We found no significant relation between birth weight and the $\gamma$-linolenic acid content of umbilical cord plasma phospholipids. Therefore, the association between cord blood $\gamma$-linolenic acid concentrations and childhood insulin resistance does not seem to be mediated through a reduction in fetal growth. According to a previous study, ${ }^{11}$ poor nutrition during late gestation could lead to permanent changes in insulin-glucose metabolism, even when the effects on intrauterine growth are small.

It is not clear how $\gamma$-linolenic acid influences insulin metabolism. Suggested mechanisms explaining the effects of dietary fatty acids on insulin metabolism involve changes in number and affinity of insulin receptors, alterations in membrane fatty acid composition, changes in the translocation of glucose transporters, changes in intracellular glucose storage, or alterations in the expression of lipogenic and glycolytic genes. ${ }^{17,19,35}$ Such mechanisms could involve the so-called proliferator-activatedreceptors (PPARs). Gamma-linolenic acid has been shown to have a relatively high affinity for PPAR $\alpha$ and PPAR $\delta$, these transcription factors playing central roles in glucose and lipid homeostasis. ${ }^{36,37}$

The observed association between a low $\gamma$-linolenic acid concentration in umbilical cord plasma phospholipids and the insulin resistance variables at age seven could reflect a genetic predisposition rather than a limited $\gamma$-linoleic acid availability dependent on maternal dietary intake. Investigators have shown that the muscle membrane fatty acid composition of children relates to maternal indices of insulin resistance. $^{38}$ 
Table 4.4: Frequency of maternal diabetes mellitus or impaired glucose tolerance during pregnancy and of family history for Type II diabetes mellitus according to tertiles of umbilical cord plasma phospholipid $\gamma$-linolenic acid concentrations at birth.

\begin{tabular}{llccc}
\multicolumn{2}{l}{ Tertile of $\gamma$-Linolenic acid } & $\begin{array}{c}\text { Maternal diabetes or IGT } \\
\text { during pregnancy }\end{array}$ & $\begin{array}{c}\text { Family history of } \\
\text { Type II diabetes }\end{array}$ & Combined \\
$(\% w t / w t)$ & $n(\%)$ & $n(\%)$ & $n(\%)$ \\
\hline$<0.04$ & $(n=85)$ & $4(5)$ & $32(38)$ & $33(39)$ \\
$0.04-0.07$ & $(n=87)$ & $3(3)$ & $28(32)$ & $30(34)$ \\
$\geq 0.07$ & $(n=87)$ & $1(1)$ & $18(21)$ & $19(22)$ \\
$P\left(\chi^{2}\right.$ square test $)$ & NS & & 0.04 & 0.04 \\
\hline
\end{tabular}

IGT, impaired glucose tolerance.

Table 4.5: Multiple regression analyses of fasting insulin concentration and insulin resistance (HOMA) in seven-year-old children with sex, current weight, birth weight, family history of diabetes and plasma phospholipid $\gamma$-linolenic acid concentrations.

\begin{tabular}{|c|c|c|c|}
\hline & Coefficient ${ }^{\prime}$ & $(95 \% \mathrm{CI})$ & $P$ \\
\hline \multicolumn{4}{|l|}{ Log insulin $(\mathrm{pmol} / \mathrm{L})$} \\
\hline $\operatorname{Sex}(0=$ boy; $1=$ girl $)$ & 3.2 & $(-0.5 ; 5.8)$ & 0.019 \\
\hline Weight (kg) & 1.0 & $(0.7 ; 1.3)$ & $<0.001$ \\
\hline Birth weight $(\mathrm{kg})$ & 0.9 & $(-2.3 ; 4.3)$ & 0.602 \\
\hline Family history of diabetes $(0=\text { no; } 1=\text { yes })^{2}$ & 30.1 & $(13.0 ; 47.3)$ & $<0.001$ \\
\hline$\gamma$-Linolenic acid at birth $\left(\%_{0} \mathrm{wt} / \mathrm{wt}\right)$ & -4.1 & $(-7.8 ;-0.4)$ & 0.028 \\
\hline$\gamma$-Linolenic acid at seven years (\%o wt/wt) & 6.6 & $(4.3 ; 8.9)$ & $<0.001$ \\
\hline Birth weight $\times$ family history & -8.8 & $(-13.9 ;-3.6)$ & $<0.001$ \\
\hline \multicolumn{4}{|l|}{ Log insulin resistance (HOMA) } \\
\hline $\operatorname{Sex}(0=$ boy; $1=$ girl $)$ & 4.2 & $(-0.3 ; 8.7)$ & 0.070 \\
\hline Weight (kg) & 1.6 & $(1.0 ; 2.1)$ & $<0.001$ \\
\hline Birth weight $(\mathrm{kg})$ & 1.7 & $(-4.1 ; 7.5)$ & 0.566 \\
\hline Family history of diabetes $(0=\text { no; } 1=\text { yes })^{2}$ & 49.6 & $(20.3 ; 78.9)$ & 0.001 \\
\hline$\gamma$-Linolenic acid at birth ( $\left.\%_{0} w t / w t\right)$ & -7.1 & $(-13.4 ;-0.8)$ & 0.028 \\
\hline$\gamma$-Linolenic acid at seven years (\%o wt/wt) & 11.1 & $(7.2 ; 15.1)$ & $<0.001$ \\
\hline Birth weight $\times$ family history & -14.3 & $(-23.0 ;-5.5)$ & 0.002 \\
\hline
\end{tabular}

HOMA, homeostasis model assessment.

'Coefficients are percentage changes in the outcome variable per unit change in predictor variables (Note that one unit change in $\gamma$-linolenic acid concentration equals $0.1 \% \mathrm{wt} / \mathrm{wt}$ ).

${ }^{2}$ Includes maternal diabetes or impaired glucose tolerance during pregnancy.

They postulate that a genetic predisposition for the incorporation, elongation, and desaturation of specific fatty acids is inherited by the child and contributes to the risk for future development of insulin resistance. ${ }^{38}$ In this study, a positive family history of diabetes was related to a lower $\gamma$-linolenic acid content of umbilical cord plasma phopholipids. However, the associations found between $\gamma$-linolenic acid concentration at birth and the insulin resistance variables at age seven were independent of family 
history. It thus seems that the associations found do not simply reflect a genetic predisposition.

The observed associations between $\gamma$-linolenic acid concentrations at birth and at seven years of age on one hand and the insulin resistance variables on the other hand could be an epiphenomenon. Insulin plays a crucial role in fatty acid metabolism and is known to enhance the activity of the enzyme delta-6-desaturase. ${ }^{39}$ Delta-6-desaturase stimulates the conversion of linoleic acid (18:2n-6) into $\gamma$-linolenic acid. A higher plasma concentration of insulin could thus explain a higher $\gamma$-linolenic acid content of plasma lipid fractions. Conversely, low levels of circulating insulin could relate to lower $\gamma$-linolenic acid concentrations. Findings in Type I (insulin-dependent) diabetes mellitus patients ${ }^{40}$ and animal models of reduced beta-cell function ${ }^{41}$ indicate that insulin deficiency relates to a lower delta-6-desaturase activity, which can be restored by insulin treatment. The associations found between plasma phospholipid $\gamma$-linolenic acid concentration and the insulin resistance variables could thus reflect such a 'mechanism' of reverse causality. Furthermore, less than $40 \%$ of the children participated during the follow-up study. Although the birth characteristics (including $\gamma$ linolenic acid concentration at birth) of the children who participated did not differ from the children who did not attend our clinic, residual bias due to selection cannot be excluded.

In conclusion, the results of the present explorative study indicate that a limited availability of $\gamma$-linolenic acid early in human life could be one of the factors involved in the fetal origins of insulin resistance.

\section{References}

1. Pyorala K. Relationship of glucose tolerance and plasma insulin to the incidence of coronary heart disease: results from two population studies in Finland. Diabetes Care. 1979;2:131-141.

2. Reaven GM. Banting lecture 1988. Role of insulin resistance in human disease. Diabetes. 1988;37:1595-1607.

3. Barker DJP, Hales CN, Fall CHD, Osmond C, Phillips K, Clark PMS. Type 2 (non-insulindependent) diabetes mellitus, hypertension, and hyperlipidaemia (syndrome $\mathrm{X}$ ): relation to reduced fetal growth. Diabetologia. 1993;36:62-67.

4. Hales CN, Barker DJP, Clark PMS, Cox L, Fall C, Osmond C, Winter PD. Fetal and infant growth and impared glucose tolerance at age 64. BMJ. 1991;303:1019-1022.

5. Lithell HO, McKeigue PM, Berglund L, Mohson R, Lithell UB, Leon DA. Relation of size at birth to non-insulin-dependent diabetes and insulin concentrations in men aged 50-60 years. BMJ. 1996;312:406-410.

6. Curhan GC, Willett WC, Rimm EB, Spiegelman D, Ascherio AL, Stampfer MJ. Birth weight and adult hypertension, diabetes mellitus, and obesity in US men. Circulation. 1996;94:3246-3250.

7. Law CM, Gordon GS, Shiell AW, Barker DJP, Hales CN. Thinness at birth and glucose tolerance in seven-year-old children. Diabet Med. 1995;12:24-29.

8. Bavdekar A, Yajnik CS, Fall CHD, Bapat S, Pandit AN, Deshpande V, Bhave S, Kellingray SD, Joglekar C. Insulin resistance syndrome in 8-year-old Indian children. Diabetes. 1999:48:24222429. 
9. Barker DJP. Mothers, babies and health in later life. Second ed. Edinburgh: Churchill Livingstone; 1998.

10. Harding JE. The nutritional basis of the fetal origins of adult disease. Int $J$ Epidemiol. 2001;30:15-23.

11. Ravelli ACJ, Meulen JHPvd, Osmond C, Barker DJP, Bleker OP. Glucose tolerance in adults after in utero exposure to the Dutch Famine. Lancet. 1998;351:173-177.

12. Dahri S, Snoeck A, Reusens-Billen B, Remacle C, Hoet JJ. Islet function in offspring of mothers on low-protein diet during gestation. Diabetes. 1991;40:115-120.

13. Langley SC, Browne RF, Jackson AA. Altered glucose tolerance in rats exposed to maternal low protein diets in utero. Comp Biochem Physiol. 1994;109:223-229.

14. Desai M, Crowther NJ, Ozanne SE, Lucas A, Hales CN. Adult glucose and lipid metabolism may be programmed during fetal life. Biochem Soc Trans. 1995;23:331-335.

15. Olson SF. Further on the association between retarded foetal growth and adult cardiovascular disease. Could low intake of marine diets be a common cause? J Clin Epidemiol. 1994;47:565569.

16. James WPT. Long-term fetal programming of body composition and longevity. Nutr Rev. 1997;55:S31-S43.

17. Storlien LH, Baur LA, Kriketos AD, Pan DA, Coony GJ, Jenkins AB, Calvet GD, Campbell LV. Dietary fats and insulin action. Diabetalogia. 1996;39:621-631.

18. Storlien LH, Kritekos AD, Calvert GD, Baur LA, Jenkins AB. Fatty acids, triglycerides and syndromes of insulin resistance. Prostagaldins Leukotr Essent Fatty Acids. 1997:57:379-385.

19. Vessby B. Dietary fat and insulin action in humans. Br J Nutr. 2000;83:S91-S96.

20. Salomaa V, Ahola I, Tuomilehto J, Aro A, Pietinen P, Korhonen HJ, Penttila I. Fatty acid composition of serum cholesterol esters in different degrees of glucose intolerance: a populationbased study. Metabolism. 1990;39:1285-1291.

21. Borkman M, Storlien LH, Pan DA, Jenkins AB, Chisholm DJ, Campbell LV. The relation between insulin sensitivity and the fatty-acid composition of skeletal-muscle phospholipids. $N$ Eng J Med. 1993;328:238-244.

22. Vessby B, Tengblad S, Lithell H. Insulin sensitivity is related to the fatty acid composition of serum lipids and skeletal muscle phospholipids in 70-year-old men. Diabetologia. 1994;37:10441050.

23. Pan DA, Lilioja S, Milner MR, Kriketos AD, Baur LA, Bogardus C, Storlien LH. Skeletal muscle membrane lipid composition is related to adiposity and insulin action. $J$ Clin Invest. 1995;96:2802-2808.

24. Vessby B, Aro A, Skarfors E, Berglund L, Salminen I, Lithell H. The risk to develop NIDDM is related to the fatty acid composition of serum chelesterol esters. Diabetes. 1994;43:1353-1357.

25. Baur LA, O'Conner J, Pan DA, Kriketos AD, Storlien LH. The fatty acid composition of skeletal muscle membrane phospholipid: its relation with the type of feeding and plasma glucose levels in young children. Metabolism. 1998;47:106-112.

26. Pettit DJ, Forman MR, Hanson RL, Knowler WC, Bennett PH. Breast feeding and incidence of non-insulin-dependent diabetes mellitus in Pima Indians. Lancet. 1997;350:166-168.

27. Rump P, Mensink RP, Kester ADM, Hornstra G. Essential fatty acid composition of plasma phospholipids and birth weight: a study in term neonates. Am J Clin Nutr. 2001;73:797-806.

28. Gerver WJM, de Bruin R. Paediatric morphometrics: a reference manual. Utrecht: Bunge; 1996.

29. Weststrate JA, Deurenberg P. Body composition in children: proposal for a method for calculating body fat percentage from total body density or skinfold-thickness measurements. $\mathrm{Am}$ J Clin Nutr. 1989:50:1104-1115.

30. Slaughter MH, Lohman TG, Boileau RA, Horswill CA, Stillman RJ, Loan MDv, Bemben DA. Skinfold equations for estimation of body fatness in children and youth. Hum Biol. 1988;60:709. 723. 
31. Al MD, Houwelingen ACv, Kester AD, Hasaart TH, de Jong AE, Hornstra G. Maternal essential fatty acid patterns during normal pregnancy and their relationship to the neonatal essential fatty acid status. Br J Nutr. 1995;74:55-68.

32. Ruige JB, Dekker JM, Nijpels G, Popp-Snijders C, Stehouwer CDA, Kostense PJ, Bouter LM. Hyperproinsulinaemia in impaired glucose tolerance is associated with a delayed insulin response to glucose. Diabetologia. 1999;42:177-180.

33. Matthews DR, Hosker JP, Rudenski AS, Naylor BA, Treacher DF, Turner RC. Homeostasis model assessment: insulin resistance and $\beta$-cell function from fasting glucose and insulin concentrations in man. Diabetologia. 1985;28:412-419.

34. Hediger ML, Overpeck MD, Kuczmarski RJ, McGlynn A, Maurer KR, Davis WW. Muscularity and fatness of infants and young children born small-for-gestational-age. Pediatrics. 1998;102:e60.

35. Clarke SD, Jump DB. Polyunsaturated fatty acid regulation of hepatic gene transcription. Lipids. 1996;31:S7-S11.

36. Xu HE, Lambert MH, Montana VG, Parks DJ, Blanchard SG, Brown PJ, Sternbach DD, Lehman JM, Wisely GB, Willson TM, Kliewer SA, Milburn MV. Molecular recognition of fatty acids by peroxisome proliferator-activated receptors. Molecular Cell. 1999;3:397-403.

37. Gervois P, Torra IP, Fruchart JC, Steals B. Regulation of lipid and lipoprotein metabolism by PPAR activators. Clin Chem Lab Med. 2000;38:3-11.

38. Baur LA, O'Connor J, Pan DA, Storlien LH. Relationships between maternal risk of insulin resistance and the child's muscle membrane fatty acid composition. Diabetes. 1999;48:112-116.

39. Brenner RR. Nutritional and hormonal factors influencing desaturation of essential fatty acids. Prog Lipid Res. 1981;20:41-47.

40. Horrobin DF. Fatty acid metabolism in health and disease: the role of $\Delta$-6-desaturase. Am J Clin Nutr. 1993;57:732S-737S.

41. Mohan IK, Das UN. Prevention of chemically induced diabetes mellitus in experimental animals by polyunsaturated fatty acids. Nutrition. 2000;17:126-151. 
Chapter 5 | Fasting triacylglycerol concentrations in seven-year-old children are related to the polyunsaturated fatty acid composition of umbilical cord plasma phospholipids at birth

Patrick Rump, Ronald P. Mensink, and Gerard Hornstra

Submitted for publication 


\begin{abstract}
Objective According to the fetal origins hypothesis, fetal nutrition is related to adult glucose intolerance and coronary heart disease. Glucose intolerance and coronary heart disease have been associated to dietary polyunsaturated fatty acids. We explored whether the availability of polyunsaturated fatty acids (PUFAs) during fetal life, as indicated by the fatty acid composition of cord blood samples, relates to childhood lipid and lipoprotein concentrations.

Design The phospholipid PUFA composition of umbilical cord blood was determined by gas-liquid chromatography in a birth-cohort of infants. At seven years of age, fasting concentrations of plasma lipids and lipoproteins were measured in 264 of these children and relations with cord plasma fatty acid concentrations were studied.

Results Umbilical cord plasma phospholipid $\gamma$-linolenic acid (18:3n-6) and dihomo- $\gamma$ linolenic acid (20:3n-6) concentrations were negatively related to fasting triacylglycerol concentrations measured at seven years of age. The highest triacylglycerol concentrations were seen in children with low 18:3n-6 and 20:3n-6 concentrations at birth and high $18: 3 n-6$ and $20: 3 n-6$ concentrations at seven years of age. Adjustment for potential confounding factors did not attenuate the associations. The fatty acid composition of umbilical cord plasma phospholipids was not related to total cholesterol, HDL cholesterol, LDL cholesterol, apolipoprotein A1, apolipoprotein $\mathrm{B}$, and lipoprotein(a) concentrations in this cohort of seven-year-old children.

Conclusion These findings could indicate that the fetal availability or metabolism of $\gamma$ linolenic acid influences the cardiovascular risk profile in children.
\end{abstract}




\section{Introduction}

A small size at birth has been associated with an increased risk for the development of adult coronary heart disease, and components of the metabolic syndrome, e.g. Type II diabetes, hypertension and dyslipidemia. ${ }^{1,2}$ Different research groups have found similar relations in both men and women from various populations. ${ }^{3.5}$ These associations led to the formulation of the fetal origins hypothesis. ${ }^{6}$ According to this hypothesis the propensity to develop coronary heart disease is a consequence of structural and functional adaptations to a limited availability of nutrients during fetal life. That fetal nutrition may indeed be involved is supported by the findings of the Dutch Hunger Winter Study and experiments in animals. ${ }^{7 \cdot 10}$ Thus far, a limited number of human studies have been conducted in an attempt to identify the nutritional factors that could play a role. These studies mainly focussed on the effects of the balance between macro-nutrients of the maternal diet during pregnancy. ${ }^{11,12}$ Hardly any work has been done to explore the importance of specific nutrients and their balance on fetal growth and later outcome.

Recently, we reported on the negative association between concentrations of $\gamma$ linolenic acid (18:3n-6) and dihomo- $\gamma$-linolenic acid (20:3n-6) in umbilical cord plasma phospholipids and components of the insulin resistance syndrome in Dutch 7year-old children. ${ }^{13}$ Children who had a lower $\gamma$-linolenic acid concentration at birth had a higher fasting concentration of insulin, proinsulin, and leptin and a higher percentage of body fat at age seven. ${ }^{13}$ These findings suggest that the fetal availability of $\gamma$-linolenic acid could be involved in the fetal origins of insulin resistance. The objective of the present study was to explore whether the umbilical cord plasma phospholipid concentration of $\gamma$-linolenic acid and dihomo- $\gamma$-linolenic acid $(20: 3 n-6)$ also relate to another component of the metabolic syndrome in 7-year-olds, i.e. their plasma lipid and lipoprotein concentrations.

\section{Subjects and Methods}

\section{Study population}

Subjects were recruited from a Dutch birth-cohort of children. As infants, these children and their mothers participated in a previous study of essential fatty acids during pregnancy and pregnancy outcome. No interventions were provided. To explore the potential long-term effects of fetal essential fatty acid availability, a follow-up study was performed between 1997 and 2000. All singleton babies born alive before 1994 and of whom an umbilical cord blood sample was available for the determination of the essential fatty acid status at birth - were candidates to be included. In total, 750 children were eligible for follow-up (for more details on this cohort see Rump et al.). ${ }^{14}$ Using the Dutch population registry, $97 \%$ of the eligible population could be traced. Three children had died and 34 lived abroad. The parents of the remaining 691 children 
received a written invitation for the follow-up evaluation. A reminder was send within fourteen days. When no response was obtained, phone-calls were made in an attempt to contact the remaining parents. Eventually we were able to contact the parents of 558 children. Of these, 297 participated, 231 refused, and 30 retracted their initial consent. Birth characteristics (i.e. birth weight, crown-heel length, occipital-frontal circumference, ponderal index, gestational age, birth order, and the $\gamma$-linolenic acid composition of umbilical cord plasma phospholipids) of the children who participated did not differ from the children who did not attend our clinic. Approval for this study was obtained from the Ethics Committee of the University Hospital Maastricht and the parents of all participating children gave their written informed consent.

\section{Data collection at follow-up}

All children were investigated at approximately seven years of age (range: $6.7-8.1$ years). The same physician (PR) examined all children and performed all venipunctures. A questionnaire and a structured interview with one or both parents were used to collect additional information. Included were information on maternal smoking during pregnancy, infant nutrition, parental education, and family history of cardiovascular diseases. Children who never received human milk were considered formula-fed and the remaining children regarded as breast-fed. Three categories of parental education were distinguished (low: primary school, lower vocational or lower general education; middle: intermediate general or intermediate vocational education; and high: higher general and higher vocational education or university). A family history of cardiovascular disease was considered when a first or second-degree family member of the child (parent, grandparent or sibling of parent) had suffered from myocardial infarction $\leq 55$ years. Anthropometric measurements were performed as described in detail by Gerver and de Bruin. ${ }^{15}$

\section{Blood sampling and laboratory analyses}

Not all parents and children agreed to a venipuncture, and no more than two puncture attempts were made. As a consequence, blood samples were obtained in 264 of the 297 children. The blood samples were collected in EDTA-treated evacuated tubes after an overnight fast. Plasma was separated from blood cells by centrifugation and stored in small portions at $-80^{\circ} \mathrm{C}$ until analysis. The fatty acid composition of umbilical cord plasma phopholipids was determined by capillary gas-liquid chromatography, as described by $\mathrm{Al}$ et al. ${ }^{16}$

All lipid and lipoprotein concentrations were measured in duplicate. Total cholesterol, triacylglycerol, and (after precipitation of apolipoprotein B containing lipoproteins) HDL cholesterol were determined enzymatically. ${ }^{17}$ The intra-assay and inter-assay variation coefficients were $1.4 \%$ and $3.0 \%$ (total cholesterol), $2.5 \%$ and $4.3 \%$ (triacylglycerol), $3.6 \%$ and $7.8 \%$ (HDL cholesterol), respectively. LDL cholesterol was calculated using the equation of Friedewald $e t$ al. ${ }^{18}$ 
Table 5.1: Neonatal and childhood characteristics of the study population by sex. ${ }^{1}$

\begin{tabular}{|c|c|c|}
\hline & $\begin{array}{c}\text { Boys } \\
(n=145)\end{array}$ & $\begin{array}{c}\text { Girls } \\
(n=119)\end{array}$ \\
\hline \multicolumn{3}{|l|}{ Neonatal characteristics } \\
\hline Length of gestation (wk) & $40.0 \quad(39.1 ; 41.0)$ & $40.1 \quad(39.1 ; 41.0)$ \\
\hline Birth weight (g) & $3375(500)$ & $3209(497)^{b}$ \\
\hline \multicolumn{3}{|l|}{ Infant nutrition } \\
\hline Formula-fed & 77 (53) & $64(54)$ \\
\hline Breast-fed & $68(47)$ & $55 \quad(46)$ \\
\hline \multicolumn{3}{|l|}{ Childhood characteristics } \\
\hline Age (y) & $7.3(0.3)$ & $7.3(0.3)$ \\
\hline Weight (kg) & $25.5(4.4)$ & $25.0(4.2)$ \\
\hline Height $(\mathrm{cm})$ & $127.7(5.0)$ & $126.3(6.1)^{a}$ \\
\hline BMI $\left(\mathrm{kg} / \mathrm{m}^{2}\right)$ & $15.5(1.9)$ & $15.6(1.7)$ \\
\hline Sum four skinfolds (mm) & $23.1 \quad(19.9 ; 26.6)$ & $26.9(23.5 ; 33.1)^{\epsilon}$ \\
\hline Total cholesterol $(\mathrm{mmol} / \mathrm{L})$ & $4.04(0.57)$ & $4.10(0.71)$ \\
\hline Triacylglycerol $(\mathrm{mmol} / \mathrm{L})$ & $0.48 \quad(0.34 ; 0.63)$ & $0.51 \quad(0.38 ; 0.72)$ \\
\hline HDL cholesterol $(\mathrm{mmol} / \mathrm{L})$ & $1.37(0.31)$ & $1.34(0.31)$ \\
\hline LDL cholesterol $(\mathrm{mmol} / \mathrm{L})$ & $2.43(0.53)$ & $2.50(0.64)$ \\
\hline LDL to HDL cholesterol ratio & $1.89(0.65)$ & $1.97(0.73)$ \\
\hline Apolipoprotein Al (mg/L) & $1365(22)$ & $1350 \quad(23)$ \\
\hline Apolipoprotein B $(\mathrm{mg} / \mathrm{L})$ & 715 (15) & 748 (17) \\
\hline Lipoprotein(a) (mg/L) & $79(17 ; 224)$ & $74(37 ; 290)$ \\
\hline
\end{tabular}

${ }^{\top}$ Values are mean (SD) or number (\%) except for gestational age, sum four skinfolds, triacylglycerol and lipoprotein(a), where values are median (inter-quartile range).

${ }^{\mathrm{a}} P<0.05,{ }^{\mathrm{b}} P<0.01,{ }^{\mathrm{c}} P<0.001$ for differences between sexes.

Apolipoprotein Al and apolipoprotein B were analyzed by an immunoturbidimetric reaction (UNI-KIT Apo Al and UNI-KIT Apo B, Hoffmann-La Roche, Basel, Switzerland). The coefficients of variation within and between assays were $2.4 \%$ and $5.1 \%$ for Apo Al, and $3.0 \%$ and $5.8 \%$ for Apo B. An enzyme-linked immonosorbent assay (ELISA) was applied to measure plasma lipoprotein(a) concentration (TintElize, Biopool, Umeå, Sweden). Variation coefficients were $7.7 \%$ and $3.4 \%$.

\section{Statistical analysis}

All analyses were restricted to the 264 children of whom a blood sample was obtained at follow-up. Data are presented as mean \pm standard deviation (SD), unless specified otherwise. Differences between groups were evaluated by unpaired two-tailed Student's $t$ tests or $\chi^{2}$ tests. Linear regression models were used to explore relations between plasma phospholipid fatty acid concentrations and lipid and lipoprotein concentrations at follow-up. Continuous variables were used instead of categories. Because of a skewed distribution of some variables (lipoprotein(a), triacylglycerol), log transformed data were used during the analyses of these variables. Multiple regression models were applied to control for potential confounding factors. A two-tailed $P$ value $<0.05$ was 
considered statistically significant. All statistical analyses were performed using STATVIEW (version 4.5, Abacus Concepts Inc., Berkeley, CA, USA).

\section{Results}

The characteristics of the study population are shown in Table 5.1. For both umbilical cord blood samples and for blood samples obtained at follow-up, the fatty acid composition of plasma phospholipids is reported in Table 5.2. The fatty acid composition of plasma phospholipids did not differ between boys and girls. See also Appendix $D$ and $E$.

Table 5.2: Fatty acids in plasma phospholipids at birth and at 7 years of age $(n=264)$.

\begin{tabular}{|c|c|c|c|c|}
\hline & \multicolumn{2}{|c|}{ At birth } & \multicolumn{2}{|c|}{ At 7 years } \\
\hline & Mean & SD & Mean & SD \\
\hline \multicolumn{5}{|l|}{ Fatty acid (\%wt/wt) } \\
\hline $18: 2 n-6$ & 7.67 & 1.29 & 23.19 & 2.27 \\
\hline $18: 3 n-6$ & 0.05 & 0.04 & 0.08 & 0.06 \\
\hline $20: 3 n-6$ & 5.18 & 0.86 & 2.97 & 0.54 \\
\hline $20: 4 n-6$ & 16.61 & 1.60 & 9.20 & 1.23 \\
\hline$\sum n-6$ & 32.16 & 1.70 & 36.78 & 1.81 \\
\hline$\sum \mathrm{n}-6 \mathrm{LCP}$ & 23.41 & 1.52 & 12.92 & 1.34 \\
\hline $18: 3 n-3$ & ND & - & 0.19 & 0.07 \\
\hline $20: 5 n-3$ & 0.23 & 0.11 & 0.51 & 0.22 \\
\hline $22: 6 n-3$ & 6.11 & 1.39 & 2.82 & 0.69 \\
\hline$\sum \mathrm{n}-3$ & 694 & 1.57 & 4.55 & 0.86 \\
\hline$\sum \mathrm{n}-3 \mathrm{LCP}$ & 6.89 & 1.57 & 4.23 & 0.87 \\
\hline$\sum S F A$ & 47.62 & 1.43 & 45.44 & 1.02 \\
\hline ¿MUFA & 11.88 & 1.65 & 13.12 & 1.38 \\
\hline ¿PUFA & 39.50 & 1.76 & 41.44 & 1.55 \\
\hline$\sum n-3 / \sum n-6$ & 0.22 & 0.05 & 0.13 & 0.03 \\
\hline Total fatty acids $(\mathrm{mg} / \mathrm{L})$ & 592 & 118 & 1060 & 148 \\
\hline
\end{tabular}

\section{Lipids, lipoproteins, and (dihomo)- $\gamma$-linolenic acid concentrations}

Triacylglycerol concentration at seven years of age was negatively associated with both $\gamma$-linolenic acid and dihomo- $\gamma$-linolenic acid concentrations measured in the phospholipid fraction of umbilical cord plasma (Table 5.3). These associations remained statistically significant when adjusted for sex, age at follow-up, and current weight. In contrast, phospholipid $\gamma$-linolenic acid and dihomo- $\gamma$-linolenic acid concentrations measured at seven years of age were positively related to childhood triacylglycerol concentrations. 
The geometric mean concentration of triacylglycerol in seven-year-old children according to tertiles of $\gamma$-linolenic acid and dihomo- $\gamma$-linolenic acid, measured at birth and at seven years of age, are depicted in Figures $5.1 \mathrm{~A}$ and B. Triacylglycerol concentration was highest in children with the lowest $\gamma$-linolenic acid and dihomo- $\gamma$ linolenic acid concentrations at birth and the highest $\gamma$-linolenic acid and dihomo- $\gamma$ linolenic acid concentrations at seven years of age.

Table 5.3: Plasma triacylglycerol concentrations in seven-year-old children according to tertiles of phospholipid $\gamma$-linolenic acid and dihomo- $\gamma$-linolenic acid concentrations in umbilical cord plasma at birth.

\begin{tabular}{lccc}
\hline Fatty acid $(\% w t / w t)$ & $\begin{array}{c}\text { Triacylglycerol } \\
(\mathrm{mmol} / \mathrm{L})^{1}\end{array}$ & Crude & Adjusted $^{3}$ \\
\cline { 3 - 4 } & & & \\
\hline -Linolenic acid & & & \\
$\quad$ Low $(<0.04)$ & $0.54 \pm 0.02$ & 0.016 & \\
$\quad$ Middle $(0.04-0.07)$ & $0.51 \pm 0.03$ & & \\
$\quad$ High $(\geq 0.07)$ & $0.44 \pm 0.02$ & & \\
Dihomo- $\gamma$-linolenic acid & & & \\
$\quad$ Low $(<4.83)$ & $0.54 \pm 0.03$ & 0.019 \\
$\quad$ Middle $(4.83-5.46)$ & $0.51 \pm 0.02$ & & \\
$\quad$ High $(\geq 5.46)$ & $0.44 \pm 0.02$ & & \\
\hline
\end{tabular}

\footnotetext{
${ }^{\top} V$ alues are geometric mean $\pm \mathrm{SE}$.

${ }^{2} P$ values are derived from simple and multiple linear regression using continuous variables.

${ }^{3}$ Adjusted for sex, age at follow-up, and current weight.
}

The difference in log-triacylglycerol concentration between children who changed from the lowest tertile of $\gamma$-linolenic acid at birth to the highest tertile at age seven and children who changed from the highest tertile of $\gamma$-linolenic acid at birth to the lowest tertile at age seven, was $34 \%$ (95\% CI: $21 \%-47 \%)$. For dihomo- $\gamma$-linolenic acid, the observed difference in log-triacylglycerol concentration between these categories was $28 \%(95 \%$ CI: $8 \%-48 \%)$. A difference of $30 \%$ in log-triacylglycerol concentration corresponds to a difference in triacylglycerol concentration of $\pm 0.30 \mathrm{mmol} / \mathrm{L}$. The proportion of children that had increased or decreased their rank order of $\gamma$-linolenic acid or dihomo- $\gamma$-linolenic acid concentration between birth and seven years of age is shown in Table 5.4 .

After correction for potential confounding factors by multiple regression analysis the association between triacylglycerol concentration at seven years of age and $\gamma$-linolenic acid or dihomo- $\gamma$-linolenic acid measured at birth remained statistically significant (Table 5.5). Children with a higher $\gamma$-linolenic acid concentration in the plasma phospholipid fraction tended to have a higher dihomo- $\gamma$-linolenic acid concentration as well $(r=0.20, P=0.001$ at birth and $r=0.33, P<0.001$ at seven years; Spearman rank correlation tests). 
A

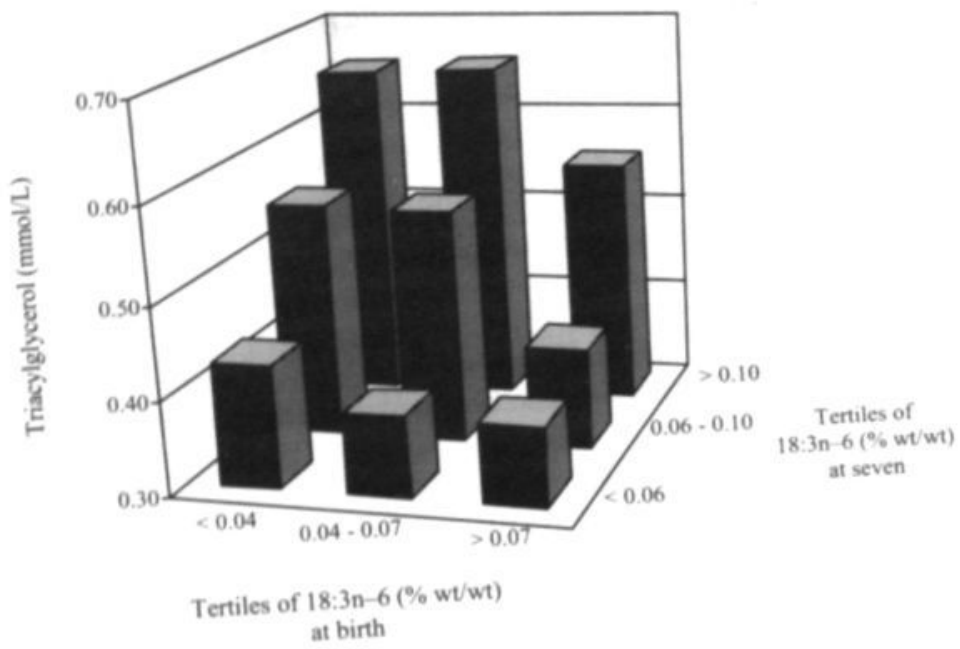

B

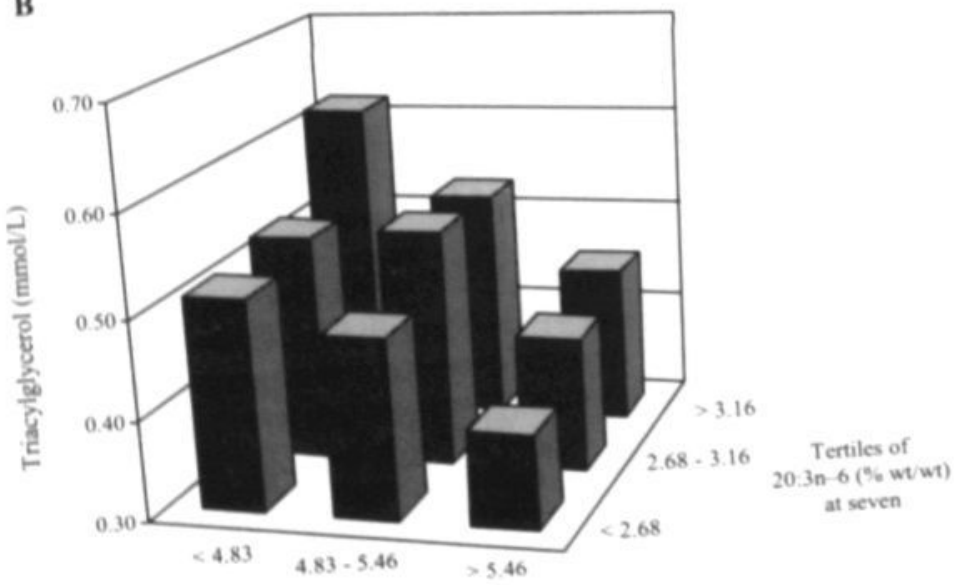

Tertiles of $20: 3 n-6(\% w t w t)$

at birth

Figure 5.1: Fasting plasma triacylglycerol concentrations in seven-year-old children according tertiles of plasma phospholipid $\gamma$-linolenic acid (A) or dihomo- $\gamma$-linolenic acid $(B)$ concentrations umbilical cord blood at birth and in blood obtained at seven years of age (n $=0.001$ for effect geometric means $(P=0.006$ for effect of $\gamma$-linolenic acid 0.001 for effect of dihomo- $\gamma$-linolenic a $\gamma$-linolenic acid concentrations at follow-up, concentrations at birth, and $P=0.002$ for effect $\log$-triacylglycerol concentration as dependent varia results from multiple regression models wid concentrations at birth and at seven years of age and $\gamma$-linolenic acid or dihomo- $\gamma$-linolenic acid concentrations at birth and at seven $y$ independent the variables). 
Triacylglycerol concentration at seven years of age was also positively related to docosahexaenoic acid concentration at birth $(P=0.02)$. This association was no longer statistically significant when length of gestation was entered as a co-variable. At seven years of age, docosahexaenoic acid concentrations did not relate to triacylglycerol levels. No relation was found between triacylglycerol levels at follow-up and umbilical cord plasma phospholipid concentrations of the other fatty acids and fatty acid groups listed in Table 5.2. Total cholesterol, HDL cholesterol, LDL cholesterol, apolipoprotein A1, apolipoprotein B, and lipoprotein(a) concentrations, measured at seven years of age, did not relate to the phospholipid fatty acid composition of umbilical cord plasma at birth. Adjustment for the fatty acid concentrations determined at seven years of age, or for sex, age at follow-up, and current weight, did not alter these results.

\section{Lipids, lipoproteins, and birth weight.}

No statistically significant association was found between birth weight and plasma triacylglycerol or the other lipid and lipoprotein concentrations measured at seven years of age, nor when adjusted for length of gestation or for sex, age at follow-up, and current weight.

Table 5.4: The proportion of children increasing, decreasing or maintaining their rank order of plasma phospholipid $\gamma$-linolenic acid and dihomo- $\gamma$-linolenic acid concentrations over a period of seven years.

\begin{tabular}{lccc} 
Tertile at birth & \multicolumn{3}{c}{ Tertile at seven years of age } \\
\cline { 2 - 4 } & Low & Middle & High \\
\hline$\gamma$-Linoleic acid & $37 \%$ & $26 \%$ & $37 \%$ \\
Low & $36 \%$ & $36 \%$ & $28 \%$ \\
$\quad$ Middle & $27 \%$ & $39 \%$ & $34 \%$ \\
$\quad$ High & & $30 \%$ & $25 \%$ \\
Dihomo- $\gamma$-linolenic acid & $45 \%$ & $36 \%$ & $30 \%$ \\
$\quad$ Low & $34 \%$ & $34 \%$ & $46 \%$ \\
Middle & $20 \%$ & & \\
High & & & \\
\hline
\end{tabular}

\section{No differences between breast-fed children and formula-fed children}

The phospholipid fatty acid concentrations of umbilical cord blood and of blood obtained at follow-up did not differ significantly between breast-fed children and formula-fed children. Total cholesterol, triacylglycerol, HDL cholesterol, LDL cholesterol, apolipoprotein A1, apolipoprotein B, and lipoprotein(a) concentrations did not differ between breast-fed children and formula-fed children either, and no statistically significant interactions were observed. 
Table 5.5: Parameters for regression of $\gamma$-linolenic acid (\%o wt/wt) and dihomo- $\gamma$-linolenic acid (\% $\mathrm{wt} / \mathrm{wt})$ concentrations in umbilical cord plasma phospholipids on log-triacylglycerol $(\mathrm{mmol} / \mathrm{L})$ at seven years of age, unadjusted and adjusted for potential confounding factors.

\begin{tabular}{|c|c|c|c|}
\hline Additional variables in model & $\beta^{1}$ & $95 \% \mathrm{CI}$ & $P$ value \\
\hline \multicolumn{4}{|l|}{$\gamma$-Linolenic acid $(18: 3 n-6)$} \\
\hline Unadjusted & -12.3 & $(-22.3 ;-2.4)$ & 0.016 \\
\hline $18: 3 n-6$ at 7 years & -13.2 & $(-22.4 ;-3.9)$ & 0.006 \\
\hline $18: 3 n-6$ at 7 years and family history ${ }^{2}$ & -12.7 & $(-21.9 ;-3.4)$ & 0.007 \\
\hline $18: 3 n-6$ at 7 years and anthropometric variables ${ }^{3}$ & -11.3 & $(-20.7 ;-2.0)$ & 0.018 \\
\hline $18: 3 n-6$ at 7 years of and socio-economic factors ${ }^{4}$ & -13.3 & $(-22.9 ;-3.6)$ & 0.007 \\
\hline $18: 3 n-6$ at 7 years and neonatal characteristics ${ }^{5}$ & -11.7 & $(-21.2 ;-2.2)$ & 0.016 \\
\hline All listed variables & -10.1 & $(-20.0 ;-0.2)$ & 0.046 \\
\hline \multicolumn{4}{|l|}{ Dihomo- $\gamma$-linolenic acid $(20: 3 n-6)$} \\
\hline Unadjusted & -5.0 & $(-9.1 ;-0.91)$ & 0.017 \\
\hline $20: 3 n-6$ at 7 years & -7.4 & $(-11.7 ;-3.1)$ & $<0.001$ \\
\hline $20: 3 n-6$ at 7 years and family history ${ }^{2}$ & -7.2 & $(-11.3 ;-2.9)$ & 0.001 \\
\hline $20: 3 n-6$ at 7 years and anthropometric variables ${ }^{3}$ & -7.9 & $(-12.2 ;-3.6)$ & $<0.001$ \\
\hline $20: 3 n-6$ at 7 years and socio-economic factors ${ }^{4}$ & -7.2 & $(-11.7 ;-2.7)$ & 0.002 \\
\hline $20: 3 n-6$ at 7 years and neonatal characteristics ${ }^{5}$ & -7.0 & $(-11.4 ;-2.5)$ & 0.002 \\
\hline All listed variables & -7.6 & $(-12.3 ;-3.0)$ & 0.001 \\
\hline
\end{tabular}

'Results of multiple regression analyses with plasma phospholipid $18: 3 n-6$ (\%o wt/wt) or $20: 3 n-6$ (\% $\mathrm{wt} / \mathrm{wt})$ at birth as independent variables and log-triacylglycerol $(\mathrm{mmol} / \mathrm{L})$ as dependent variable. The regression coefficients are percentage changes in the outcome variable per unit change in 18:3n-6 or 20:3n-6 concentration at birth (Note that for 18:3n-6 one unit equals $0.1 \% \mathrm{wt} / \mathrm{wt}$ and that for $20: 3 \mathrm{n}-6$ one unit equals $1 \% \mathrm{wt} / \mathrm{wt}$ ).

${ }^{2}$ Family history of cardiovascular disease (dichotomous; yes vs no).

'Anthropometric variables: height, weight, and sum four skinfolds

'Socio-economic factors: maternal and paternal education (entered as dummy variables) and number of children in household.

'Neonatal characteristics: birth weight, length of gestation, maternal smoking during pregnancy (dichotomous; yes vs no) and infant nutrition (dichotomous; breast-fed $v s$ formula-fed).

\section{Discussion}

In the present study, childhood triacylglycerol concentration appeared related to concentrations of $\gamma$-linolenic acid and its elongation product dihomo- $\gamma$-linolenic acid measured seven years earlier in umbilical cord blood. Both $\gamma$-linolenic acid and dihomo- $\gamma$-linolenic acid can be derived from the parent essential fatty acid linoleic acid $(18: 2 n-6)$, the most abundant PUFA in a typical Western-style diet. A high linoleic acid intake does, however, not necessarily guarantee a higher availability of these metabolites. ${ }^{19}$ In contrast, an increased intake of $\gamma$-linolenic acid results in an increased concentration of $\gamma$-linolenic acid and dihomo- $\gamma$-linolenic acid in blood lipid fractions. ${ }^{19}$ Dietary $\gamma$-linolenic acid may thus be a more important source of $\gamma$-linolenic acid and dihomo- $\gamma$-linolenic acid than linoleic acid. The presented data seem to suggest that a low intrauterine availability of $\gamma$-linolenic acid predispose individuals to higher 
triacylglycerol concentrations in childhood. An elevated plasma triacylglycerol concentration is increasingly recognized as an important component of the metabolic syndrome associated with the risk for atherosclerosis and the development of cardiovascular diseases. ${ }^{20-22}$

We have previously shown that a lower umbilical plasma concentration of $\gamma$ linolenic acid also relates to higher fasting insulin and proinsulin concentrations in 7 year-olds. ${ }^{13}$ Circulating triacylglycerol concentration is closely related to insulin sensitivity. ${ }^{23}$ The association found between triacylglycerol and umbilical cord blood $\gamma$ linolenic acid concentration could be explained by an alteration in insulin metabolism. How $\gamma$-linolenic acid may influence insulin metabolism, however, is not clear. Maybe peroxisome proliferator-activated receptors (PPARs) are involved. ${ }^{24}$ Interestingly, gamma-linolenic acid has been shown to have a relatively high affinity for PPAR $\alpha$ and PPAR $\delta$, these transcription factors are involved in glucose and lipid homeostasis. ${ }^{25.26}$

The positive associations found between triacylglycerol concentration and phospholipid $\gamma$-linolenic acid and dihomo- $\gamma$-linolenic acid concentrations measured at seven years of age, are consistent with previous findings in adults and children. ${ }^{27.28}$ Why fasting triacylglycerol concentration is negatively related to the $\gamma$-linolenic acid and dihomo- $\gamma$-linolenic acid content of umbilical cord plasma and positively related to the $\gamma$-linolenic acid and dihomo- $\gamma$-linolenic acid content of plasma phospholipids at age seven is not known. Whether this reflects different effects of $\gamma$-linolenic acid depending on the moment of exposure, an adaptation to a low $\gamma$-linolenic acid availability in fetal life with the inability to handle a higher availability during childhood, or some other mechanism needs further exploration.

Although birth weight is positively related with umbilical cord plasma phospholipid dihomo- $\gamma$-linolenic acid concentration in this cohort, ${ }^{14}$ no relation was found between birth weight and childhood plasma triacylglycerol and other lipid and lipoprotein concentrations. We also found no significant relation between birth weight and the $\gamma$-linolenic acid content of umbilical cord plasma phospholipids. It thus seems that $\gamma$-linolenic acid availability early in life relates to triacylglycerol concentration later in life independent of attained weight at birth. According to Ravelli et al., ${ }^{8}$ a limited availability of nutritients during late gestation may lead to permanent changes in insulin metabolism, even when the effects on birth weight are small.

The phospholipid fatty acid composition of umbilical cord blood collected after birth is similar to that of fetal blood collected in utero at a comparable gestational age, ${ }^{29}$ but it does not necessarily reflect the PUFA availability during earlier stages of pregnancy. This may be of relevance since exposure to the Dutch famine during early gestation, rather than during late gestation, has been associated to adult plasma cholesterol and lipoprotein concentrations. ${ }^{30}$ This could explain why we did not find a relation with cholesterol and lipoprotein levels.

The use of umbilical cord plasma phospholipid fatty acid composition as a biomarker for fetal PUFA availability has limitations. The PUFA content of cord 
plasma phospholipids depends upon a complex of different factors, which include maternal PUFA intake, maternal PUFA reserves and placental PUFA delivery, in combination with endogenous maternal, placental and fetal fatty acid metabolism. We can not differentiate between these factors. Moreover, we can not exclude the possibility of reverse causality. Alterations in insulin metabolism by other unknown factors could influence both plasma triacylglycerol concentration and, through effects on delta-6-desaturase, the $\gamma$-linolenic acid and dihomo- $\gamma$-linolenic acid content of plasma phospholipids. Another limitation of the present study is the fact that less than $40 \%$ of the eligible children participated. Although the birth characteristics, including $\gamma$-linolenic acid and dihomo- $\gamma$-linolenic acid concentration in umbilical plasma, did not differ between those who participated and those who did not attend our clinic, residual confounding due to selection can not be fully excluded. Controlled intervention studies are therefore needed to establish whether the availability of $\gamma$-linolenic acid is indeed the primary factor involved and whether the quality of fat intake during pregnancy in women could have an influence on the cardiovascular risk profile in her offspring.

\section{References}

1. Barker DJP, Winter PD, Osmond C, Margetts B, Simmonds SJ. Weight in infancy and death from ischaemic heart disease. Lancet. 1989;ii:577-580.

2. Barker DJP, Hales CN, Fall CHD, Osmond C, Phillips K, Clark PMS. Type 2 (non-insulindependent) diabetes mellitus, hypertension, and hyperlipidaemia (syndrome $\mathrm{X}$ ): relation to reduced fetal growth. Diabetologia. 1993;36:62-67.

3. Frankel S, Elwood P, Sweetnam P, Yarnell J, Davy-Smith G. Birth weight, body mass index in middle-age, and incident coronary heart disease. Lancet. 1996;348:1478-1480.

4. Curhan GC, Willett WC, Rimm EB, Spiegelman D, Ascherio AL, Stampfer MJ. Birth weight and adult hypertension, diabetes mellitus, and obesity in US men. Circulation. 1996;94:32463250.

5. Leon DA, Lithell HO, Vagero D, Koupilova I, Mohsen R, Berglund L, Lithell UB, McKeigue PM. Reduced fetal growth rate and increased risk of death from ischaemic heart disease: cohort study of 15000 Swedish men and women born 1915-29. BMJ. 1998;317:241-245.

6. Barker DJP. Mothers, babies and health in later life. Second ed. Edinburgh: Churchill Livingstone; 1998.

7. Ravellı GP, Stein ZA, Susser MW. Obesity in young men after famine exposure in utero and early pregnancy. $N$ Engl J Med. 1976;7:349-354.

8. Ravelli ACJ, Meulen JHPvd, Osmond C, Barker DJP, Bleker OP. Glucose tolerance in adults after in utero exposure to the Dutch Famine. Lancet. 1998;351:173-177.

9. Dahri S, Snoeck A, Reusens-Billen B, Remacle C, Hoet JJ. Islet function in offspring of mothers on low-protein diet during gestation. Diabetes. 1991;40:115-120.

10. Woodall SM, Johnston BM, Breier BH, Gluckman PD. Chronic maternal undernutrition in the rat leads to delayed postnatal growth and elevated blood pressure of offspring. Pediatr Res. 1996:40:438-443.

11. Godfrey KM, Robinson S, Barker DJP, Osmond C. Maternal nutrition in early and late pregnancy in relation to placental and fetal growth. BMJ. 1996:312:410-414. 
12. Campbell DM, Hall MH, Barker DJP, Cross J, Shiell AW, Godfrey KM. Diet in pregnancy and the offspring's blood pressure 40 years later. Br J Obstet Gynaecol. 1996;103:273-280.

13. Rump P, Popp-Snijders C, Heine RJ, Hornstra G. Components of the insulin resistance syndrome in seven-year-old children: relations with birth weight and the polyunsaturated fatty acid content of umbilical cord plasma phospholipids. Diabetologia. 2002;45:349-355.

14. Rump P, Mensink RP, Kester ADM, Hornstra G. Essential fatty acid composition of plasma phospholipids and birth weight: a study in term neonates. Am J Clin Nutr. 2001;73:797-806.

15. Gerver WJM, de Bruin R. Paediatric morphometrics: a reference mamual. Utrecht: Bunge; 1996.

16. Al MD, Houwelingen ACv, Kester AD, Hasaart TH, de Jong AE, Hornstra G. Maternal essential fatty acid patterns during normal pregnancy and their relationship to the neonatal essential fatty acid status. Br J Nutr. 1995;74:55-68.

17. Plat J, Mensink RP. Vegetable oil based versus wood based stanol ester mixtures: effects on serum lipids and hemostatic factors in non-hypercholesterolemic subjects. Atherosclerosis. 2000;148:101-112.

18. Friedewald WT, Levy RI, Fredrickson DS. Estimation of the concentration of low-density lipoprotein cholesterol in plasma, without use of the preparative ultracentrifuge. Clin Chem. 1972;18:499-502.

19. Manku MS, Morse-Fisher N, Horrobin DF. Changes in human plasma essential fatty acid levels as a result of administration of linoleic acid and gamma-linolenic acid. Eur $J$ Clin Nutr. 1988:42:55-60.

20. Gotto AM. Triglyceride as a risk factor for coronary artery disease. Am $J$ Cardiol. 1998;82:22Q-25Q.

21. Austin MA, Hokanson JE, Edwards KL. Hypertriglyceridemia as a cardiovascular risk factor. Am J Cardiol. 1998;81:7B-12B.

22. Hokanson JE, Austin MA. Plasma triglyceride level is a risk factor for cardiovascular disease independent of high-density lipoprotein cholesterol level: a meta-analysis of population based prospective studies. J Cardiovasc Risk. 1996;3:213-219.

23. Reaven GM. Insulin resistance, hyperinsulinemia, hypertriglyceridemia and hypertension: parallels between human disease and rodent models. Diabetes Care. 1991;14:195-202.

24. Clarke SD, Jump DB. Polyunsaturated fatty acid regulation of hepatic gene transcription. Lipids. 1996;31:S7-S11.

25. Xu HE, Lambert MH, Montana VG, Parks DJ, Blanchard SG, Brown PJ, Sternbach DD, Lehman JM, Wisely GB, Willson TM, Kliewer SA, Milburn MV. Molecular recognition of fatty acids by peroxisome proliferator-activated receptors. Molecular Cell. 1999;3:397-403.

26. Gervois P, Torra IP, Fruchart JC, Steals B. Regulation of lipid and lipoprotein metabolism by PPAR activators. Clin Chem Lab Med. 2000;38:3-11.

27. Boberg M, Vessby B, Croon LB. Fatty acid composition of platelets and plasma lipid esters in relation to platelet function in patients with ischeamic heart disease. Atherosclerosis. 1985;58:49-63.

28. Moilanen T, Solakivi-Jaakkola T, Viikari J, Răsănen L, Åkerblom HK, Uhari M, Pasanen M, Nikkari T. Fatty acid composition of serum cholesteryl esters in relation to serum lipids and apolipoproteins in 3-18-year-old Finnish children and adolescents. Atherosclerosis. 1986;59:113-119.

29. Houwelingen ACv, Foreman-van Drongelen MMHP, Nicolini U, Nicolaides KH, Al MDM, Kester ADM, Hornstra G. Essential fatty acid status of fetal plasma phospholipids: similar to postnatal values obtained at comparable gestational ages. Early Hum Dev. 1996;46:141-152.

30. Roseboom TJ, Meulen JHPvd, Osmond C, Barker DJP, Ravelli ACJ, Bleker OP. Plasma lipid profile in adults after prenatal exposure to famine. Am J Clin Nutr. 2000;72:1101-1106. 
Chapter 5 
Chapter 6 I Interaction between a common variant of the cholesteryl ester transfer protein gene and the apolipoprotein $\mathbf{E}$ polymorphism: effects on plasma lipids and lipoproteins in a cohort of seven-yearold children

Patrick Rump, Ronald P. Mensink, and Gerard Hornstra

Nutr Metab Cardiovasc Dis (in press) 


\begin{abstract}
Background/Aim Common variations in genes, like apolipoprotein E (apo E) and cholesteryl ester transfer protein (CETP), are important determinants of plasma lipid and lipoprotein levels. Both, apo E and CETP are contributing to the reverse transport of cholesterol back to the liver. Therefore, the effects of variations at the CETP locus may very well interact with apo E genotype.

Methods \& Results As part of an ongoing study, the combined effects of apo E genotype and heterogeneity at the CETP gene locus on plasma lipid and lipoproteins were studied in a birth-cohort-based sample of 257 Dutch prepubescent boys and girls (aged $6.7-8.1$ years). Children with an apo E2E3 genotype (carrying the $\varepsilon 2$ allele; $\arg _{158} \rightarrow$ cys) had lower concentrations of LDL cholesterol and apolipoprotein B (apo B) than those with an apo E4E3 genotype (carrying the $\varepsilon 4$ allele; cys ${ }_{112} \rightarrow$ arg) or apo E3E3 genotype (homozygous for the parent $\varepsilon 3$ allele). These associations were statistically significant in children who were homozygous $(P=0.004$ for LDL; $P=$ 0.002 for apo B) or heterozygous $(P<0.0001$; for LDL and apo B) for the absence of the Taq IB polymorphism at the CETP gene locus (B2 allele), but not in those homozygous for the presence of this variant (B1B1). The highest plasma HDL cholesterol concentrations were observed in children with the CETP B2B2 genotype. The difference in HDL cholesterol level between CETP genotype groups was statistically significant only in E2E3 carriers $(P=0.01)$. The LDL/HDL ratio was significantly lower in E2E3 carriers, but not when combined with a CETP B1B1 genotype.

Conclusion Our findings indicate that apo E genotype and heterogeneity at the CETP gene locus have an additive and interactive influence on plasma lipid and lipoprotein levels in children.
\end{abstract}




\section{Introduction}

High serum concentrations of low-density lipoprotein (LDL) cholesterol and low concentrations of high-density lipoprotein (HDL) cholesterol are associated with an increased risk for the development of coronary heart disease in adults. ' Childhood levels of lipids and lipoproteins persist over time and tend to predict adult values. ${ }^{2,3}$ More importantly, childhood levels of LDL and HDL cholesterol are related to the presence and severity of asymptomatic atherosclerosis of the aorta and coronary arteries. ${ }^{4.5}$ Common variations at the apolipoprotein E (apo E) gene locus are important determinants of plasma lipid and lipoprotein levels in adults and children. ${ }^{6-8}$ Variation at the apo E locus may also influence the serum lipid and lipoprotein changes from childhood into adulthood. ${ }^{9}$ Moreover, apo E genotype seems to predispose individuals to develop coronary heart disease later in life. ${ }^{10}$

Three common apo $\mathrm{E}$ isoforms are recognized: the $\varepsilon 2$ allele ( $\arg _{158} \rightarrow$ cys), the $\varepsilon 4$ allele $\left(\mathrm{cys}_{112} \rightarrow \mathrm{arg}\right)$, and the parent $\varepsilon 3$ allele. The $\varepsilon 2$ allele is associated with lower total cholesterol, LDL cholesterol and apolipoprotein B (apo B) concentrations, whereas the $\varepsilon 4$ allele relates to higher levels. ${ }^{6-8}$ Higher concentrations of HDL cholesterol have been found in subjects carrying the $\varepsilon 2$ allele. "These findings have been attributed to a reduced affinity of apo E2 for the apo E/remnant-receptor, leading to a reduced clearance of cholesterol from apo E-containing lipoproteins (e.g. HDL, chylomicrons, very-low-density lipoprotein) by the liver. ${ }^{12} \mathrm{~A}$ compensatory upregulation of the apo B/E LDL-receptor, resulting in an enhanced hepatic uptake of LDL, may explain the lower LDL cholesterol and apo B concentrations seen in $\varepsilon 2$ carriers. $^{12}$ Apo E is also considered a contributor to reverse cholesterol transport; the transport of cell-derived cholesterol back to the liver mediated by apo E-containing HDL particles. ${ }^{13,14}$ Genetic variation at the apo E locus may influence this process. ${ }^{15,16}$

Variation at the apo E gene locus has also been associated with the metabolic response to dietary cholesterol, including changes in the plasma concentrations of an enzyme called cholesteryl ester transfer protein (CETP). ${ }^{17}$ CETP plays a key role in reverse cholesterol transport. It mediates the transfer of cholesteryl esters from HDL to apo $\mathrm{B}$ containing lipoproteins like LDL and very-low-density lipoprotein (VLDL), in exchange for triacylglycerol. ${ }^{18-20}$ In adults, plasma CETP concentrations, CETP activity and HDL cholesterol levels have been associated with common variations in the CETP gene, like the so-called Taq IB polymorphism. ${ }^{21-23}$ The presence of this polymorphism (i.e. $\mathrm{B} 1$ allele) has also been related to the progression of coronary atherosclerosis. ${ }^{24}$

So far, limited information is available on the relations between the Taq IB CETP polymorphism and plasma lipid and lipoprotein concentrations in children. ${ }^{25}$ The study of genotype-phenotype relationships in children is important since it allows the observation of associations in the absence of atherosclerotic diseases and adult life style factors that may obscure or alter such relations, like smoking and alcohol consumption. ${ }^{20}$ Considering the role of apo $\mathrm{E}$ in the hepatic clearance of cholesterol 
from apo E containing lipoproteins like $\mathrm{HDL}$ and as a contributor to reverse cholesterol transport, the effects of variations at the CETP locus may very well interact with apo E genotype. Therefore, we investigated the association between CETP genotype and fasting plasma lipid and lipoprotein concentrations in children and whether these associations were modulated by variations at the apo E gene locus.

\section{Subjects and Methods}

\section{Study population}

Subjects were recruited from a Dutch birth-cohort of children. As infants, these children and their mothers participated in a previous observational study of plasma essential fatty acid concentrations during pregnancy and their relation to pregnancy outcome. No interventions were provided during this study. To explore the potential long-term effects of fetal essential fatty acid availability on the cardiovascular risk profile of children, a follow-up study was performed between 1997 and 2000.

In total, 750 children were eligible for follow-up (for more details on this cohort see Rump et al. .). With the aid of the Dutch population registry $97 \%$ of the eligible population could be traced. Three of the children had died and 34 lived abroad. The remaining 691 children and their parents were invited for the follow-up evaluation. Of these, 297 participated, 231 refused, 133 did not respond, and 30 did not show up at appointments made or retracted their initial consent. Birth characteristics (i.e. birth weight, birth length, head circumference, ponderal index, length of gestation and birth order) of the children who participated did not differ from the children who did not attend our clinic (data not shown).

Genomic DNA was available in 257 ( 141 boys and 116 girls) of the participating children. The present analyses focus on the combined effects of apo E and CETP genotype on plasma lipid and lipoprotein concentrations in children and was therefore limited to these 257 subjects. Approval for this study was obtained from the Ethics Committee of the University Hospital in Maastricht and the parents of all participating children gave their written, informed consent.

\section{Data collection at follow-up}

All children were investigated at approximately seven years of age. Mean age (range) at follow-up was $7.3(6.7-8.1)$ years. The same physician (PR) examined all children and performed all venipunctures. Anthropometric measurements were performed as described in detail by Gerver and de Bruin. ${ }^{27}$ Blood samples were collected in EDTAtreated evacuated tubes after an overnight fast. Plasma was separated from blood cells by centrifugation and stored in small portions at $-80^{\circ} \mathrm{C}$ until analysis.

Total cholesterol, triacylglycerol, and (after precipitation of apo B containing lipoproteins) HDL cholesterol were determined enzymatically, as described before. ${ }^{28}$ The intra-assay and inter-assay variation coefficients were $1.4 \%$ and $3.0 \%$ (total 
cholesterol), $2.5 \%$ and $4.3 \%$ (triacylglycerols), $3.6 \%$ and $7.8 \%$ (HDL cholesterol), respectively. LDL cholesterol was calculated using the equation of Friedewald $e t$ al. ${ }^{29}$ Apolipoprotein Al (apo Al) and apolipoprotein B (apo B) were analyzed by an immunoturbidimetric reaction (UNI-KIT Apo Al and UNI-KIT Apo B, Hoffmann-La Roche, Basel, Switzerland). The coefficients of variation within and between assays were $2.4 \%$ and $5.1 \%$ for apo $\mathrm{Al}$ and $3.0 \%$ and $5.8 \%$ for apo B. An enzyme-linked immonosorbent assay (ELISA) was applied to measure plasma $\mathrm{Lp}(\mathrm{a})$ concentration (TintElize, Biopool, Umeå, Sweden). Variation coefficients were $7.7 \%$ and $3.4 \%$.

\section{DNA isolation and apo E and CETP genotyping}

Genomic DNA was extracted from isolated leukocytes using standard procedures. ${ }^{28}$ The Taq IB polymorphism of the CETP gene and apo E genotypes were determined by PCR and subsequent restriction-fragment-length polymorphism analysis as described by Kuivenhoven et al. ${ }^{22}$ and Plat and Mensink, ${ }^{28}$ respectively. After repeated attempts, the apo E genotype could not be determined in 6 children and the CETP genotype could not be determined in 16 children.

\section{Statistical analysis}

Data are presented as mean \pm SE. Associations of apo E and CETP genotypes with plasma lipid and lipoprotein concentrations and the demographic characteristics weight, height and BMI were evaluated by one-way analysis of variance (ANOVA). E2E2 and E2E3 genotypes (E2 group) and the E4E4 and E4E3 genotypes (E4 group) were compared to the E3E3 genotype. The distributions of boys and girls among apo E and CETP genotype groups was investigated with chi-square tests. The interaction between apo E genotype and CETP genotype was studied using stratified analyses, and when significant $(P<0.05$ by ANOVA) effects were found, subsequent post-hoc pair-wise comparisons between the individual genotype groups were performed (using a Bonferoni correction for multiple testing; $\alpha=0.0165$ ). Because of their low frequency and unequal distribution among CETP genotype groups, subjects with the rare apo E genotypes E2E2 $(n=2)$ and E4E4 $(n=3)$ were excluded during the analysis of interaction with CETP genotype. Children with the E2E4 genotype $(n=10)$ were excluded during all analyses because they carry an allele that is common to both the E2 group and the E4 group. Because of a skewed distribution of $\mathrm{Lp}(\mathrm{a})$ and triacylglycerol concentrations, log-transformed values were used. For these two variables the reported means are geometric means. The assumption of a Hardy-Weinberg equilibrium for the distribution of apo E and CETP genotypes was tested using gene counting and chisquare analysis. 


\section{Results}

\section{Frequency of apo E and CETP polymorphisms}

The allele frequencies of the apo E polymorphism were $0.10,0.76$ and 0.14 for the $\varepsilon 2$, $\varepsilon 3$ and $\varepsilon 4$ alleles respectively. The CETP gene B1 and B2 alleles were found at frequencies of 0.55 and 0.45 . For both the apo E and the CETP polymorphism, the observed genotype frequencies were in Hardy-Weinberg equilibrium. The frequencies of the CETP genotypes B1B1, B1B2 and B2B2 did not differ between the E2E3, E3E3 and E4E3 genotype groups ( $P=0.44$ by chi-square analysis).

\section{Plasma lipids, lipoproteins and Apo E or CETP genotype.}

Plasma lipid and lipoprotein concentrations did not differ between boys and girls. There were no statistically significant differences between the apo E and CETP genotype groups with respect to the distribution of sex, height, weight and BMI (not shown). There was a clear association of apo E and CETP genotypes with plasma lipid and lipoprotein concentrations (Table 6.1). The lowest levels of total cholesterol, LDL cholesterol, LDL/HDL cholesterol ratio, apo B and the highest concentration of HDL cholesterol were seen in the apo E2 genotype group. Concentrations of apo A1 did not differ significantly between the apo E genotype groups, but there was a tendency of a higher apo A1 level in E2 carriers. Variation at the CETP locus was associated to plasma total cholesterol, LDL cholesterol, HDL cholesterol, apo B and apo A1 concentrations (Table 6.1). The highest LDL cholesterol and apo B concentrations and the highest LDL/HDL cholesterol ratio were found in children with the B1B1 genotype. The highest HDL cholesterol and apo A1 concentrations were found in B2B2 carriers. In this population, triacylglycerol and $\mathrm{Lp}(\mathrm{a})$ concentrations did not differ among the apo $\mathrm{E}$ and CETP genotype groups.

\section{Combined effects of Apo E and CETP genotypes}

The associations between the Taq IB polymorphism in the CETP gene and plasma lipid and lipoprotein concentrations were most pronounced in subjects with an apo E E2E3 genotype. Children with a CETP B1B1 genotype had a statistically significant higher apo B concentration than children with a B1B2 or B2B2 genotype, but only when combined with the apo E2E3 genotype (Figure 6.1). Apo B concentrations did not differ between CETP genotype groups in children with the apo E E3E3 and E4E3 genotypes (Figure 6.1). Conversely, a statistically significant lower apo B concentration in E2E3 carriers compared to E3E3 and E4E3 carriers was seen only when combined with a B1B2 or a B2B2 CETP genotype (Figure 6.1). The differences in apo B concentration were not statistically significant between the apo $\mathrm{E}$ genotype groups in children with a CETP B1B1 genotype. 
Table 6.1: Total cholesterol, high-density lipoprotein (HDL) cholesterol, low-density lipoprotein (LDL) cholesterol, LDL HDL cholesterol ratio, apolipoprotein Al (apo A1), apolipoprotein B (apo B), triacylglycerol and lipoprotein (a) (Lp(a)) concentrations in a cohort of seven-year-old children according to genetic variation at either the apolipoprotein E (apo E) or the cholesteryl ester transfer protein (CETP) loci. ${ }^{1}$

\begin{tabular}{|c|c|c|c|c|}
\hline & \multicolumn{3}{|c|}{ Apo E genotype ${ }^{2}$} & \multirow[t]{2}{*}{$P$} \\
\hline & $\begin{array}{l}\text { E2 group } \\
(n=38)\end{array}$ & $\begin{array}{c}\text { E3E3 } \\
(n=146)\end{array}$ & $\begin{array}{c}\text { E4 group } \\
(n=57)\end{array}$ & \\
\hline Total cholesterol $(\mathrm{mmol} / \mathrm{L})$ & $3.73 \pm 0.08^{2.6}$ & $4.12 \pm 0.05^{2}$ & $4.24 \pm 0.08^{b}$ & 0.0002 \\
\hline HDL cholesterol $(\mathrm{mmol} / \mathrm{L})$ & $1.46 \pm 0.05^{2}$ & $1.32 \pm 0.03^{\mathrm{a}}$ & $1.38 \pm 0.04$ & 0.03 \\
\hline LDL cholesterol (mmol/L) & $2.01 \pm 0.08^{a, b}$ & $2.53 \pm 0.05^{\star}$ & $2.62 \pm 0.07^{b}$ & $<0.0001$ \\
\hline LDL/HDL cholesterol ratio & $1.47 \pm 0.09^{a b}$ & $2.04 \pm 0.06^{\circ}$ & $1.98 \pm 0.08^{b}$ & $<0.0001$ \\
\hline Apo Al (mg/L) & $1434 \pm 35$ & $1346 \pm 18$ & $1342 \pm 28$ & 0.07 \\
\hline Apo B $(\mathrm{mg} / \mathrm{L})$ & $625 \pm 28^{2 . b}$ & $755 \pm 13^{2}$ & $760 \pm 16^{b}$ & $<0.0001$ \\
\hline Triacylglycerols (mmol/L) & $0.51 \pm 0.04$ & $0.50 \pm 0.02$ & $0.48 \pm 0.03$ & 0.79 \\
\hline \multirow[t]{3}{*}{$\operatorname{Lp}(a)(m g / L)$} & $72 \pm 16$ & $80 \pm 9$ & $68 \pm 15$ & 0.75 \\
\hline & \multicolumn{3}{|c|}{ CETP genotype } & $P$ \\
\hline & $\begin{array}{c}\text { B1B1 } \\
(n=76)\end{array}$ & $\begin{array}{c}\text { B1B2 } \\
(n=113)\end{array}$ & $\begin{array}{c}\text { B2B2 } \\
(n=52)\end{array}$ & \\
\hline Total cholesterol $(\mathrm{mmol} / \mathrm{L})$ & $4.15 \pm 0.07$ & $3.93 \pm 0.06$ & $4.17 \pm 0.09$ & 0.02 \\
\hline HDL cholesterol $(\mathrm{mmol} / \mathrm{L})$ & $1.28 \pm 0.03^{\mathrm{a}}$ & $1.36 \pm 0.03$ & $1.45 \pm 0.05^{a}$ & 0.009 \\
\hline LDL cholesterol $(\mathrm{mmol} / \mathrm{L})$ & $2.62 \pm 0.07^{2}$ & $2.33 \pm 0.05^{a}$ & $2.43 \pm 0.08$ & 0.002 \\
\hline LDL/HDL cholesterol ratio & $2.15 \pm 0.08^{a, b}$ & $1.81 \pm 0.06^{\mathrm{a}}$ & $1.82 \pm 0.10^{b}$ & 0.001 \\
\hline Apo Al (mg/L) & $1343 \pm 24$ & $1325 \pm 20^{a}$ & $1439 \pm 36^{4}$ & 0.009 \\
\hline Apo B $(\mathrm{mg} / \mathrm{L})$ & $782 \pm 19^{a}$ & $689 \pm 14^{2}$ & $739 \pm 20$ & 0.0003 \\
\hline Triacylglycerols (mmol/L) & $0.51 \pm 0.02$ & $0.47 \pm 0.02$ & $0.55 \pm 0.04$ & 0.12 \\
\hline $\mathrm{Lp}(\mathrm{a})(\mathrm{mg} / \mathrm{L})$ & $75 \pm 11$ & $65 \pm 10$ & $71 \pm 15$ & 0.79 \\
\hline
\end{tabular}

${ }^{1}$ Values are means $\pm \mathrm{SE}$, except for triacylglycerol and $\mathrm{Lp}(\mathrm{a})$ concentrations (geometric means $\pm \mathrm{SE}$ ).

${ }^{2}$ E2 group, E2E2 and E2E3 carriers; E4 group, E4E3 and E4E4 carriers; subjects with the E2E4 genotype $(n=10)$ were excluded.

${ }^{2 . b} P<0.0165$ for pair-wise differences between genotype groups indicated by the same number.

Plasma HDL cholesterol and LDL cholesterol levels differed significantly between the CETP genotype groups only in children with an apo E2E3 genotype (Table 6.2). The $P$ value for the difference in LDL cholesterol between E2E3/B1B1 and E2E3/B2B2 carriers was 0.025 , and thus just above our pre-defined criterion of statistical significance $(\alpha=0.0165)$. Conversely, LDL cholesterol concentrations were significantly lower in E2E3 carriers compared to both E3E3 and E4E3 carriers in children with a CETP B1B2 or B2B2 genotype (Table 6.2). Additionally, the higher concentration of HDL cholesterol observed in apo E2 carriers was most pronounced in subjects carrying a CETP B2 allele. However, the differences in plasma HDL cholesterol levels between apo E genotype groups was statistically significant in B2B2 carriers only (Table 6.2). The ratio of LDL cholesterol to HDL cholesterol was 
significantly lower in E2E3 carriers except when combined with a CETP B1B1 genotype (Table 6.2). The $P$ value for the difference in LDL/HDL ratio between $\mathrm{E} 2 \mathrm{E} 3 / \mathrm{B} 2 \mathrm{~B} 2$ and E4E3/B2B2 carriers was 0.022 , slightly higher than our pre-defined criterion of statistical significance $(\alpha=0.0165)$. In children with an apo E2E3 genotype, apo A1 concentrations for B1B1, B1B2, and B2B2 carriers were $1407 \pm 63$, $1361 \pm 45$, and $1556 \pm 76 \mathrm{mg} / \mathrm{L}$, respectively $(P$ value $=0.08$, by ANOVA $)$. Apo A1 concentrations for $\mathrm{B} 1 \mathrm{~B} 1, \mathrm{~B} 1 \mathrm{~B} 2$, and $\mathrm{B} 2 \mathrm{~B} 2$ carriers were $1310 \pm 30,1343 \pm 27$, and $1405 \pm 55 \mathrm{mg} / \mathrm{L}$ for E3E3 carriers and $1328 \pm 69,1298 \pm 38$, and $1416 \pm 64 \mathrm{mg} / \mathrm{L}$ for E4E3 carriers, respectively (ANOVA $P$ value $>0.2$ for both). Concentrations of triacylglycerol and $\mathrm{Lp}(\mathrm{a})$ did not differ significantly between the stratified CETP and apo E genotype groups (not shown).

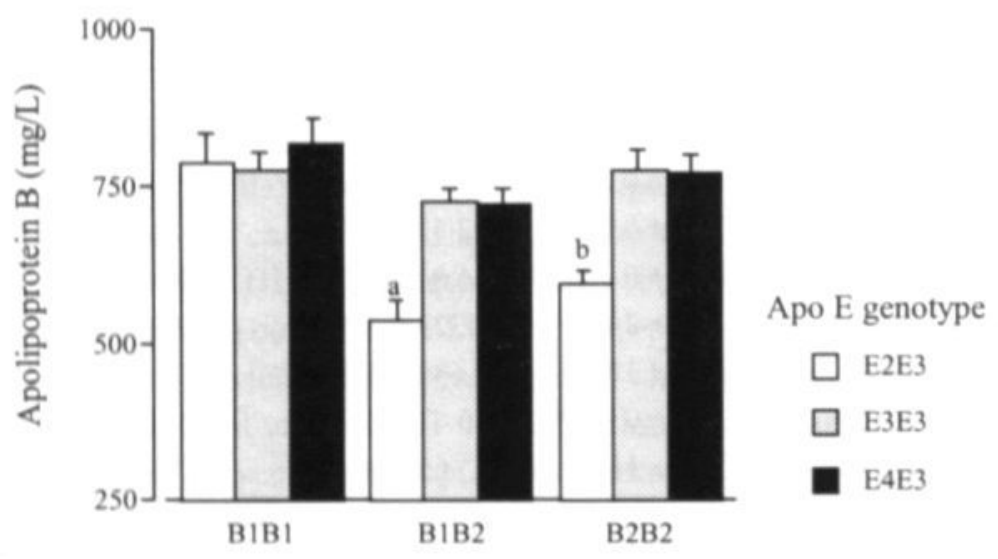

CETP genotype

Figure 6.1: Mean ( \pm SE) plasma apolipoprotein B concentration in seven-year-old children according to combinations of genetic variation at the cholesteryl ester transfer protein (CETP) and apolipoprotein E (apo E) loci. Subjects with the rare apo E genotypes E2E2 $(n=2)$, E4E4 $(n=3)$, and E2E4 $(n=10)$ were excluded. The difference in apolipoprotein B concentration between CETP genotype groups was statistically significant in apo E2E3 carriers only $(P<0.0001$; ANOVA). The difference in apolipoprotein B concentration between apo E genotype groups was statistically significant in children with a CETP B1B2 or B2B2 genotype $\left(P<0.0001\right.$ and $P=0.002$, respectively; ANOVA). ${ }^{2} P<0.0165$ for difference with $\mathrm{B} 1 \mathrm{~B} 2 \mathrm{E} 3 \mathrm{E} 3, \mathrm{~B} 1 \mathrm{~B} 2 \mathrm{E} 4 \mathrm{E} 3$, and $\mathrm{B} 1 \mathrm{~B} 1 / \mathrm{E} 2 \mathrm{E} 3$. ${ }^{\mathrm{b}} \mathrm{P}<0.0165$ for difference with $\mathrm{B} 2 \mathrm{~B} 2 / \mathrm{E} 3 \mathrm{E} 3, \mathrm{~B} 2 \mathrm{~B} 2 \mathrm{E} 4 \mathrm{E} 3$, and B1B1/E2E3. 


\section{Discussion}

Our findings indicate an additive and interactive effect of heterogeneity at the CETP gene locus and the apo E genotype on plasma lipid and lipoprotein concentrations in children. The apo E and CETP allele frequencies found in the present study were similar to previous reported frequencies in Dutch adult populations. ${ }^{24,30}$ According to Hubacek et al., ${ }^{25}$ common polymorphisms in the CETP gene do not determine plasma lipid levels during childhood. This conclusion was based on their finding that CETP Taq IB allele frequencies did not differ between Czechoslovakian children from opposite ends of the cholesterol distribution curve. ${ }^{25}$ In the present cohort, total cholesterol concentrations in B1 homozygotes were indeed similar to those in B2 homozygotes. However, the observed associations between the CETP Taq IB polymorphism and plasma HDL cholesterol and apo Al levels in our cohort of sevenyear-olds, which are consistent with previous findings in adult populations, ${ }^{21-23}$ indicate that this CETP gene variation does relate to plasma lipid and lipoprotein levels in children. The relations found might be explained by the increased concentration and activity of circulating CETP associated with this polymorphism. ${ }^{22.23}$ It should be emphasized, however, that the Taq IB polymorphism itself is not located in a coding region of the CETP gene. ${ }^{22}$ This polymorphism could be a non-functional marker in close linkage disequilibrium with a mutation elsewhere in the CETP gene or in nearby genes. For instance, the recently described $-629 \mathrm{~A} / \mathrm{C}$ polymorphism located in the promoter region of the CETP gene, which is tightly linked with the Taq IB polymorphism. $^{31}$ Alternatively, the Taq IB polymorphism may have an effect on transcriptional regulation of the CETP gene.

The observation that the association between CETP genotype and plasma lipids and lipoproteins is most pronounced in apo $\varepsilon 2$ carriers is new. Plasma CETP activity is positively related to apo $\mathrm{E}$ concentrations ${ }^{32}$ and apo $\mathrm{E}$ concentrations are higher in subjects with an E2E3 genotype. ${ }^{12}$ Further, higher plasma concentrations of CETP and lower levels of HDL cholesteroi are found in response to a high-cholesterol diet in adults carrying an $\varepsilon 2$ allele. ${ }^{17}$ Maybe due to such an increased CETP responsiveness, the impact of heterogeneity at the CETP gene locus is larger in $\varepsilon 2$ carriers. The relations between the apo $\varepsilon 2$ allele and plasma LDL and HDL cholesterol concentrations has been attributed to the reduced affinity of apo E2 to the apo E/remnant-receptor and a compensatory up-regulation of the apo B/E LDL-receptor, resulting in an increased clearance of the apo B-containing LDL particles. ${ }^{12} \mathrm{~A}$ higher CETP activity may lead to higher concentrations of LDL cholesterol and apo B. ${ }^{33}$ This could explain the higher LDL cholesterol and apo B concentrations in $\varepsilon 2$ carriers with a B1B1 genotype when compared to children with a B1B2 or B2B2 genotype. This association between the CETP Taq IB polymorphism and plasma levels of apo B and LDL cholesterol is generally not observed in adult populations. The reason for this 
difference between children and adults is not clear, maybe the absence of adult life style factors like alcohol consumption or smoking play a role. ${ }^{20}$

In transgenic mice expressing a defective apo E (like apo E2), co-expression of CETP leads to an increase of cholesteryl esters in apo B containing lipoproteins. ${ }^{34}$ Additionally, an increase in the concentration of plasma apo B and (VLDL + LDL) cholesterol concentrations has been observed in mice over-expressing CETP. ${ }^{35}$ This increase in apo $\mathrm{B}$ concentration could be a consequence of a down-regulation of the apo B/E LDL-receptor. ${ }^{18.36}$ The lower apo B and LDL cholesterol concentrations in $\varepsilon 2$ carriers, generally explained by an up-regulation of this particular receptor, might thus in theory be compensated by an increased CETP activity such as associated with the B1B1 genotype.

Table 6.2: High-density lipoprotein (HDL) cholesterol, low-density lipoprotein (LDL) cholesterol, and the LDL/HDL cholesterol ratio in a cohort of seven-year-old children according to combinations of genetic variation at the apolipoprotein $\mathrm{E}$ and the cholesteryl ester transfer protein (CETP) loci.'

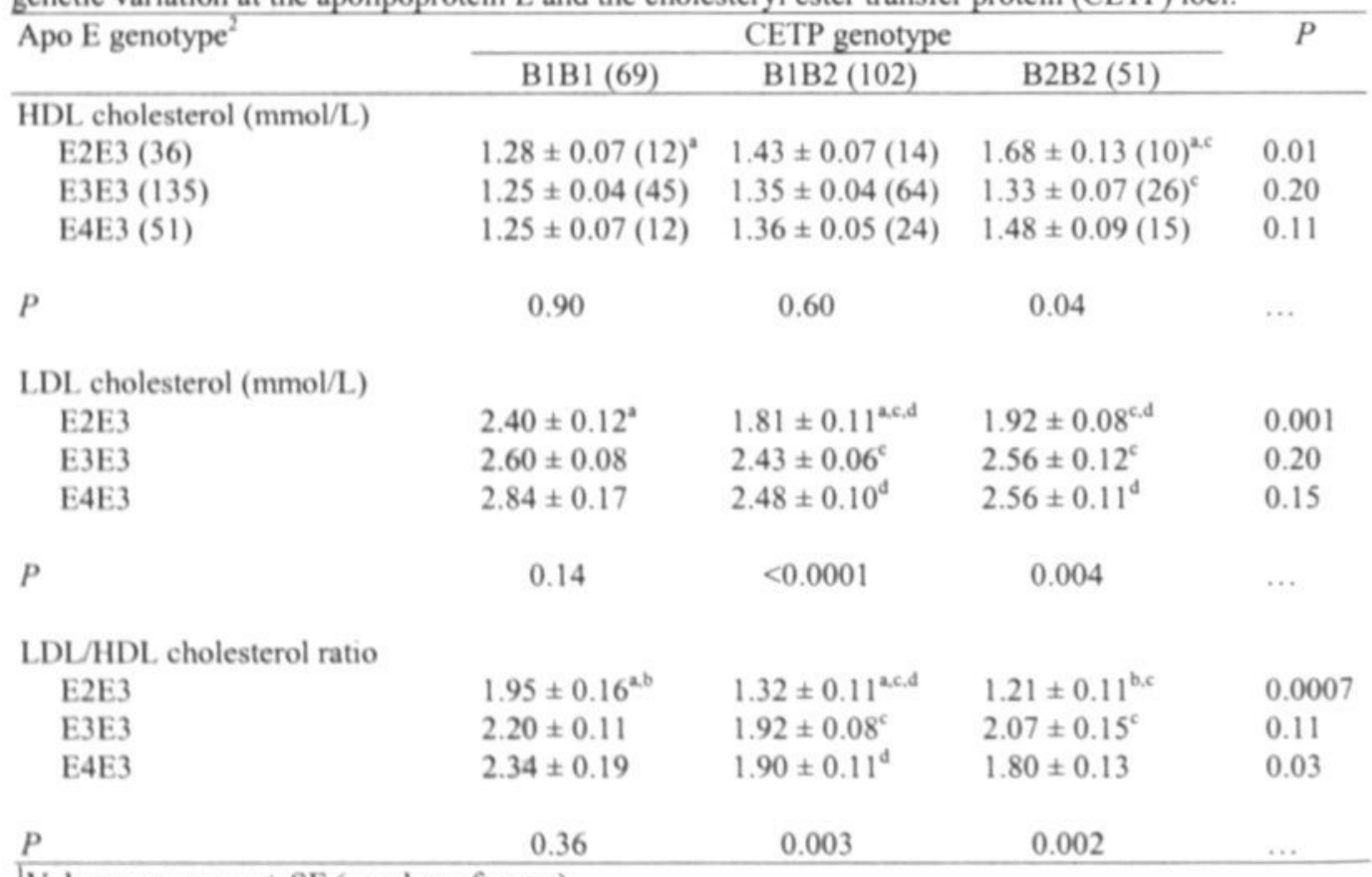

TValues are means \pm SE (number of cases).

'Subjects with the apo E genotypes E2E2 $(n=2), \mathrm{E} 4 \mathrm{E} 4(n=3)$ and E2E4 $(n=10)$ were excluded.

${ }^{2 . b} P<0.0165$ for pair-wise differences between CETP genotype groups indicated by the same number (horizontally).

e.d $P<0.0165$ for pair-wise differences between 2 , $\mathrm{E}$ gerotype groups indicated by the same letter (vertically). 
In mice, overexpression of CETP also resulted in a higher (VLDL + LDL) cholesterol/HDL cholesterol ratio, which was related to an increase in the size of atherosclerotic lesions in the aorta. ${ }^{37}$ In the present study, the highest mean LDL/HDL cholesterol ratios were seen in children carrying the CETP B1B1 genotype.

Because of the small sample size, we were unable to study the effects of CETP genotype in children homozygous for $\varepsilon 2$. This is of interest since children homozygous for $\varepsilon 2$ may develop type III hyperlipoproteinemia (dysbetalipoproteinemia). ${ }^{38}$ Our observations in children heterozygous for the $\varepsilon 2$ allele suggest that a genetically based increase in CETP activity (such as associated with a B1B1 genotype) may indeed be an additional factor in the development of this phenotype, as proposed by Fazio and colleagues based on their experiments in mice. ${ }^{34}$ Whether an interaction between apo $\mathrm{E}$ and CETP genotype, as seen in the present study, is also observed in adults, remains to be explored. The observed interaction between CETP and apo E genotype may also have implications for the prevention of cardiovascular diseases. Interventions designed to reduce the activity of CETP (e.g. newly developed CETP inhibitors $)^{39,40}$ may provide interesting approaches in lowering LDL cholesterol and increasing HDL cholesterol, especially in subjects with a genetic predisposition to a high CETP activity. Our results seem to indicate that the effectiveness of such interventions may depend upon apo $\mathrm{E}$ genotype.

\section{References}

1. Grundy SM. Cholesterol and coronary heart disease. Future directions. JAMA. 1990;264:30533059.

2. Mahoney LT, Lauer RM, Lee J, Clarke WR. Factors affecting tracking of coronary heart disease risk factors in children. The Muscatine Study. Ann N Y Acad Sci. 1991;623:120-132.

3. Webber LS, Srinivasan SR, Wattigney WA, Berenson GS. Tracking of serum lipids and lipoproteins from childhood to adulthood: The Bogalusa Heart Study. Am J Epidemiol. 1991:133:884-899.

4. Mahoney LT, Burns TL, Stanford W, Thompson BH, Witt JD, Rost CA, Lauer RM. Coronary risk factors measured in childhood and young adult life are associated with coronary artery calcification in young adults: the Muscatine Study. J Am Coll Cardiol. 1996;27:277-284.

5. Berenson GS, Srinivasan SR, Bao W, Newman WPr, Tracy RE, Wattingney WA. Association between multiple cardiovascular risk factors and atherosclerosis in children and young adults. The Bogalusa Heart Study. N Engl J Med. 1998;338:1650-1606.

6. Howard BV, Gidding SS, Liu K. Association of apolipoprotein E phenotype with plasma lipoproteins in African-American and white young adults. The CARDIA Study. $\mathrm{Am} J$ Epidemiol. 1998;148:859-868.

7. Smit M, Knijff Pd, Rosseneu M, Bury J, Klasen E, Frants R, Havekes LM. Apolipoprotein E polymorphism in the Netherlands and its effects on plasma lipid and apolipoprotein levels. Hum Genet. 1988;80:287-292.

8. Sanghera DK, Ferrell RE, Aston CE, McAllister AE, Kamboh MI, Kimm SYS. Quantitative effects of the apolipoprotein E polymorphism in a biracial sample of 9-10-year-old girls. Atherosclerosis. 1996;126:35-42. 
9. Srinivasan SR, Ehnholm C, Elkasabany A, Berenson G. Influence of apolipoprotein E polymorphism on serum lipids and lipoprotein changes from childhood to adulthood. The Bogalusa Heart Study. Atherosclerosis. 1999;143:435-443.

10. Wilson PWF, Schaefer EJ, Larson MG, Ordovas JM. Apolipoprotein E alleles and risk of coronary disease: a meta-analysis. Arterioscler Thromb Vasc Biol. 1996;16:1250-1255.

11. Srinivasan SR, Ehnholm C, Wattigney WA, Bao W, Berenson GS. The relation of apolipoprotein E polymorphism to multiple cardiovascular risk in children: the Bogalusa Heart Study. Atherosclerosis. 1996;123:33-42.

12. Utermann G. Apolipoprotein E polymorphism in health and disease. Am Heart J. 1987;113:433-440.

13. Eckardstein Av, Assmann G. High density lipoprotein and reverse cholesterol transport: lessons from mutations. Atherosclerosis. 1998;137:S7-S11.

14. Eckardstein Av, Hofer JR, Assmann G. High density lipoproteins and arteriosclerosis: role of cholesterol efflux and reverse cholesterol transport. Arterioscler Thromb Vasc Biol. 2001;21:13-27.

15. Huang Y, Eckardstein Av, Wu S, Assmann G. Effects of the apolipoprotein E polymorphism on uptake and transfer of cell-derived cholesterol. J Clin Invest. 1995;96:2693-2701.

16. Cullen P, Cignarella A, Brennhausen B, Mohr S, Assmann G, Eckardstein Av. Phenotypedependent differences in apolipoprotein E metabolism and cholesterol homeostasis in human monocyte-derived macrophages. J Clin Invest. 1998;101:1670-1677.

17. Martin LJ, Connolly PW, Nancoo D, Wood N, Zhang ZJ, Maguire G, Quinet E, Tall AR, Marcel YL, McPherson R. Cholesteryl ester transfer protein and high density lipoprotein responses to cholesterol feeding in men: relationship to apolipoprotein E genotype. $J$ Lipid Res. 1993;34:437-446.

18. Moulin P. Cholesteryl ester transfer protein: an enigmatic protein. Horm Res. 1996;45:238-244.

19. Bruce C, Chouinard RA, Tall AR. Plasma lipid transfer proteins, high-density lipoproteins, and reverse cholesterol transport. Annu Rev Nutr. 1998;18:297-330.

20. Yamashita S, Hirano K, Sakai N, Matsuzawa Y. Molecular biology and pathophysiological aspects of plasma cholesteryl ester transfer protein. Biochim Biophys Acta. 2000;1529:257-275.

21. Kondo I, Berg K, Drayna D, Lawn R. DNA polymorphism at the locus for human cholesteryl ester transfer protein (CETP) is associated with high density lipoprotein cholesterol and apolipoprotein levels. Clin Genet. 1989;35:49-56.

22. Kuivenhoven JA, Knijf Pd, Boer JMA, Smalheer HA, Botma GJ, Seidell JC, Kastelein JJP, Pritchard PH. Heterogeneity at the CETP gene locus. Influence on plasma CETP concentrations and HDL cholesterol levels. Aterioscler Thromb Vasc Biol. 1997;17:560-568.

23. Gudnason V, Kakko S, Nicaud V, Savolainen MJ, Kesäniemi YA, Tahvanainen E, Humphries S. Cholesteryl ester transfer protein gene effect on CETP activity and plasma high-density lipoprotein in European populations. Eur J Clin Invest. 1999;29:116-128.

24. Kuivenhoven JA, Jukema JW, Zwinderman AH, Knijff Pd, McPherson R, Bruschke AVG, Lie $\mathrm{KI}$, Kastelein JJP. The role of a common variant of the cholesteryl ester transfer protein gene in the progression of coronary atherosclerosis. $N$ Eng J Med. 1998;338:86-93.

25. Hubacek JA, Pistulkova H, Skodova Z, Lanska V, Poledne R. Polymorphisms in genes for cholesterol ester transfer protein, apolipoprotein C-III and lipoprotein lipase in children with high and low cholesterol levels [in Czech]. Cas Lek Cesk. 2001;140:79-81.

26. Rump P, Mensink RP, Kester ADM, Hornstra G. Essential fatty acid composition of plasma phospholipids and birth weight: a study in term neonates. Am J Clin Nutr. 2001;73:797-806.

27. Gerver WJM, de Bruin R. Paediatric morphometrics: a reference manual. Utrecht: Bunge; 1996. 
28. Plat J, Mensink RP. Vegetable oil based versus wood based stanol ester mixtures: effects on serum lipids and hemostatic factors in non-hypercholesterolemic subjects. Atherosclerosis. 2000; 148:101-112.

29. Friedewald WT, Levy RI, Fredrickson DS. Estimation of the concentration of low-density lipoprotein cholesterol in plasma, without use of the preparative ultracentrifuge. Clin Chem. 1972;18:499-502.

30. Boer LMA, Feskens EJM, Schouten EG, Havekes LM, Seidell JC, Kromhout D. Lipid profiles reflecting high and low risk for coronary heart disease: contribution of apolipoprotein E polymorphism and lifestyle. Atherosclerosis. 1998;136.

31. Dachet C, Poirier O, Cambien F, Rouis M. New functional promoter polymorphism, CETP/629 , in cholesteryl ester transfer protein (CETP) gene related to CETP mass and high density lipoprotein cholesterol levels: role of $\mathrm{Spl} / \mathrm{Sp} 3$ in transcriptional regulation. Arterioscler Thromb Vasc Biol. 2000;20:507-515.

32. McPherson R, Mann CJ, Tall AR, Hogue M, Martin L, Milne RW, Marcel YL. Plasma concentrations of cholesteryl ester transfer protein in hyperlipoproteinemia. Relation to cholesteryl ester transfer protein activity and other lipoprotein variables. Arterioscler Thromb. 1991;11:797-804.

33. Tato F, Vega GL, Tall AR, Grundy SM. Relation between cholesteryl ester transfer protein activities and lipoprotein cholesterol in patients with hypercholesterolemia and combined hyperlipidemia. Arterioscler Thromb Vasc Biol. 1995;15:112-120.

34. Fazio S, Marotti KR, Lee YL, Castle CK, Melchior GW, Rall SC. Coexpression of cholesteryl ester transfer protein and defective apolipoprotein $\mathrm{E}$ in transgenic mice alters plasma cholesterol distribution. Implications for the pathogenesis of type III hyperlipoproteinemia. $J$ Biol Chem. 1994;269:32368-32372.

35. Marotti KR, Caslte CK, Murray EF, Rehberg HG, Polites HG, Melchior GW. The role of cholesteryl ester transfer protein in primate aoplipoprotein A-I metabolism; insights from studies with tansgenic mice. Arterioscler Thromb. 1992;12:736-744.

36. Liu J, Zhang YL, Spence MJ, Vestal RE, Wallace PM, Grass DS. Liver LDL receptor mRNA expression is decreased in human apo B/CETP double transgenic mice and is regulated by diet as well as the cytokine oncostatin M. Arterioscler Thromb Vasc Biol. 1997;17:2948-2954.

37. Marotti KR, Castle CK, Boyle TP, Lin AH, Murray RW, Melchior GW. Severe atherosclerosis in transgenic mice expressing simian cholesteryl ester transfer protein. Nature. 1993;364:73-75.

38. Kwiterovich PO. Diagnosis and management of familial dyslipoproteinemia in children and adolescents. Pediatr Clin North Am. 1990;37:1489-1523.

39. Kothari HV, Poirier KJ, Lee WH, Satoh Y. Inhibition of cholesterol ester transfer protein by CGS 25159 and changes in lipoproteins in hamsters. Atherosclerosis. 1997;128:59-66.

40. Bisgaier CL, Essenburg AD, Minton LL, Homan R, Blankley CJ, White A. Cholesterol ester transfer protein inhibition by PD 140195. Lipids. 1994;29:811-818. 
Chapter 6 
Chapter 7 $\quad$ General discussion 


\section{Introduction}

A low weight at birth is associated with an increased risk for the development of adult heart disease, Type II diabetes mellitus and related risk factors such as hypertension and dyslipidemia. This relationship between infant weight at birth and adult diseases led to the formulation of the fetal origins hypothesis. ${ }^{1}$ According to this hypothesis, the propensity to develop coronary heart disease, Type II diabetes mellitus, hypertension and other disorders are consequences of intra-uterine adaptations to fetal undernourishment. ${ }^{2}$ The risk for adult diseases may thus be "programmed" or "imprinted" by a limited and/or unbalanced availability of nutrients in utero. ${ }^{3,4}$ Only a small number of human studies have been conducted to identify the nutrients involved. One group of nutrients that has been implicated, but has not been studied in this context so far, are essential fatty acids and their longer-chain, more-unsaturated derivatives, abbreviated as LCP's (long-chain polyenes).$^{5-7}$ Beneficial effects of dietary essential fatty acids have been described in relation to adult cardiovascular diseases and related disorders. Whether the availability of these substances during fetal life may influence later cardiovascular risk is not known. The primary objective of the study described in the present thesis was therefore to explore whether the availability of essential fatty acids and their longer-chain more-unsaturated derivatives during fetal live relates to the cardiovascular risk profile of children. For this purpose we studied cardiovascular risk indicators in a cohort of Dutch children with known essential fatty acid status at birth.

\section{Main findings of the study}

\section{Essential fatty acids and birth weight}

The first step in exploring the potential role of essential fatty acids in the fetal origins of cardiovascular diseases was to study relations between birth weight and the fatty acid concentrations in umbilical cord blood samples. Previous studies had demonstrated that infants born with a lower weight also have a lower essential fatty acid status at birth. ${ }^{8-13}$ These findings suggest that the availability of essential fatty acids could be a determinant of fetal growth. Especially arachidonic acid $(20: 4 n-6)$ is considered an important fatty acid for growth. ${ }^{9,10,13.14}$ However, these studies included mostly premature infants and low-birth-weight neonates. Therefore, confirmation of these findings for infants born at term was needed. In the study described in Chapter 3 we investigated the relation between birth weight and the essential fatty acid concentrations measured in umbilical cord plasma phospholipids of the neonates from our cohort. Surprisingly, the proportion of arachidonic acid and docosahexaenoic acid (22:6n-3) in umbilical cord plasma phospholipids was negatively related to neonatal weight at birth. This finding is in total contrast with previous observations in premature infants and low-birth-weight neonates. Other long-chain polyene concentrations (docosatetraenoic acid $(22: 4 n-6)$, docosapentaenoic acid $(22: 5 n-3), \Sigma n-6$ LCPs, and $\Sigma n-3$ LCPs) were 
also negatively related to infant size at birth (Chapter 3). Additionally, markers for the general essential fatty acid status (like Mead acid, the ratio of $\sum n-3+n-6$ to $\sum n-7+n-$ 9 fatty acids, and the ratio $22: 6 n-3$ to $22: 5 n-6$ ) indicated a lower essential fatty acid status in larger neonates rather than in smaller neonates. Therefore, these findings suggest that, in term neonates, a high weight at birth is related to a lower biochemical essential fatty acid status in general. An explanation for the inconsistency with studies in premature infants and low-birth-weight neonates could be that additional (pathologic) factors associated with premature birth or severe growth retardation affected both essential fatty acid status and intrauterine growth in these populations. Another explanation could be a lack of an appropriate adjustment for gestational age at birth (see Chapter 3).

In contrast to arachidonic acid and docosahexaenoic acid, concentrations of dihomo- $\gamma$-linolenic acid in umbilical cord blood were positively related to size at birth. The positive association between dihomo- $\gamma$-linolenic acid concentration and size at birth seems more consistent than those reported for arachidonic acid and docosahexaenoic acid. Higher concentrations of dihomo- $\gamma$-linolenic acid in blood lipid fractions or vessel walls of larger neonates have been reported previously and have been found in premature infants as well. ${ }^{911,15-17}$ Maybe dihomo- $\gamma$-linolenic acid is important for intrauterine growth. No study has yet evaluated the effect of dihomo- $\gamma$ linolenic acid supplementation (or supplementation of its precursor $\gamma$-linolenic acid) on intrauterine or postnatal growth. In fact, the suggestion that arachidonic acid and docosahexaenoic acid are determinants of neonatal growth have not been supported by intervention studies either. The most cited intervention study is the study performed by Carlson et al. ${ }^{14}$ In this trial a negative effect of fish oil supplemented formula (rich in docosahexaenoic acid and eicosapentaenoic acid) on infant growth had been found. ${ }^{14}$ The importance of arachidonic acid was suggested based on the observed reduction in blood arachidonic acid concentrations accompanying this intervention. ${ }^{18}$ However, an alternative explanation could be that the supplemented $n-3$ fatty acids themselves inhibited growth. Recent intervention studies in term neonates did not find any effect of docosahexaenoic acid supplementation (with or without arachidonic acid) on postnatal growth. ${ }^{19-22}$ Studies using formulas with the addition of arachidonic acid alone are lacking. Firm conclusions on cause and effect cannot be drawn from observed associations; and since conclusive evidence from controlled intervention studies is also lacking it is difficult to uphold the suggestion that essential fatty acids like arachidonic acid have a stimulatory effect on fetal growth, even in premature infants.

The relative concentrations of $n-3$ and $n-6$ long-chain polyenes in maternal plasma phospholipids decreases during the second half of pregnancy. ${ }^{23}$ In the present study, this decrease was found to be more pronounced in mothers of larger infants (Chapter 3). The second half of pregnancy is considered an important period for fetal essential fatty acid accretion. Between 37 and 42 weeks of gestational age, the essential fatty acid status in cord blood samples still increases. Especially the proportion of the 
n-3 long-chain polyene docosahexaenoic acid increases in umbilical cord blood during this period (Chapter $2 \& 3$ ). A heavier infant probably accretes more long-chain polyenes than a smaller neonate does. The larger decrease in plasma long-chain polyene concentrations of mothers of heavier babies might thus be explained by an increased mother-to-fetal transport, driven by an increased fetal accretion of these substances. If this is true, the lower LCP concentrations found in heavier neonates could suggest that this increase in the maternal-to-fetal LCP flux is not sufficient to maintain cord plasma long-chain polyene levels and maybe fetal essential fatty acid status. On the other hand, the lower long-chain polyene concentrations in fetal blood of heavier neonates may result from the fact that these substances are divided over larger body stores.

\section{Essential fatty acids and cardiovascular risk indicators in children}

Consistent relations between umbilical cord blood fatty acid concentrations and cardiovascular risk variables measured at age seven were only found for $\gamma$-linolenic acid and its direct elongation product dihomo- $\gamma$-linolenic acid (Chapter $4 \&$ Chapter 5). A lower concentration of $\gamma$-linolenic acid in umbilical cord blood related to higher plasma insulin, proinsulin, leptin, and triacylglycerol concentrations and to a higher percentage of body fat at age seven. A lower dihomo- $\gamma$-linolenic acid concentration at birth also related to higher plasma insulin and triacylglycerol concentrations in seven-year-olds. These variables are all components of the so-called insulin resistance syndrome. Our findings thus seem to indicate a relation between the availability of $\gamma$-linolenic acid and/or dihomo- $\gamma$-linolenic acid during fetal life and the development of insulin resistance during childhood. This suggestion is supported by the fact that concentrations for both fatty acids in umbilical cord blood were negatively related with the homeostasis model assessment (HOMA) index for insulin resistance. Based on the absence of consistent relationships, the availability of other more abundant essential fatty acids (i.e. linoleic acid, arachidonic acid and docosahexaenoic acid) does not seem to be an important factor in the development of an adverse cardiovascular risk profile during childhood.

\section{Birth weight and cardiovascular risk indicators in children}

Similar to previous studies conducted in adults and children, ${ }^{24-30}$ we found that (after correction for sex, age, current weight and length of gestation) a small size at birth related to higher levels of the insulin resistance variables and an increased body fatness later in life (Table 7.1 and Figure 7.1). In our study, fasting insulin concentration and the HOMA index for insulin resistance were highest in children who had a low birth weight in combination with a low umbilical cord plasma phospholipid $\gamma$-linolenic acid concentration (Chapter 4). After adjustment for birth weight, cord blood $\gamma$-linoleic acid concentration remained associated with HOMA insulin resistance and fasting insulin levels. We found no significant relation between birth weight and the $\gamma$-linolenic acid content of umbilical cord plasma phospholipids. These findings indicate that the 
relations between $\gamma$-linolenic acid and the risk variables are not be mediated through an effect on fetal growth. It seems that the effects of birth weight and that of the fetal availability of $\gamma$-linolenic acid are additive rather than interactive. According to the findings of the Dutch Hunger Winter study, ${ }^{31}$ the availability of nutrients during late gestation could still be important even when the effects on intrauterine growth are small.

\section{Gene polymorphisms}

Common genetic variations can be important predictors of circulating cardiovascular risk factor levels in children and may influence the response of a subject to an environmental stimulus. The potential effects of fetal essential fatty acid availability on the cardiovascular risk profile of children could therefore be dependent upon the presence of such gene variants. In the present study, two common gene polymorphisms were investigated (Chapter 6). Their selection was based on a relatively high population frequency of the rare allele and on the fact that relations between their presence and plasma lipid concentrations had been reported several times before. These polymorphisms were the apolipoprotein E polymorphism ( $\varepsilon 2$ allele: $\arg _{158} \rightarrow$ cys; $\varepsilon 4$ allele: cys ${ }_{112} \rightarrow$ arg; and the parent $\varepsilon 3$ allele) and the Taq IB polymorphism in the cholesteryl transfer protein gene (CETP) gene (B1 allele: presence of Taq IB site in intron 1; B2 allele: absence of Taq IB site). The apo E $\varepsilon 2$ allele is associated with lower total cholesterol, LDL cholesterol and apolipoprotein B (apo B) concentrations, whereas the $\varepsilon 4$ allele relates to higher levels. ${ }^{32-34}$ Higher plasma CETP concentrations, an increased CETP activity and lower HDL cholesterol levels are associated with the CETP Taq IB B1 allele. ${ }^{35-37}$

The selected polymorphisms in the apo E and CETP genes were indeed important predictors of circulating lipid and lipoprotein concentrations in children (Chapter 6). Additionally, we could demonstrate a genotype-genotype interaction between these common gene variants, especially in relation to plasma apolipoprotein B concentration (Chapter 6). Our primary aim, however, was to investigate the influence of these common genetic variations on the potential association between essential fatty acid concentrations in umbilical cord blood on one hand and the plasma lipid and lipoprotein concentrations measured at age seven on the other hand. No statistically significant relations were found between essential fatty acid concentrations at birth and the lipid and lipoprotein concentrations determined at age seven, except for triacylglycerol (Chapter 5). Triacylglycerol concentrations were, however, not related to the studied variations in the apo E and CETP gene (Chapter 6). Therefore, the two selected polymorphisms were not informative in relation to our hypothesis (i.e. that potential effects of fetal essential fatty acid availability on the cardiovascular risk profile of children could be modulated by the presence of common gene variants). 
Table 7.1: Unadjusted mean anthropometric measurements and biochemical parameters in 7-year-old children according to birth weight.

\begin{tabular}{|c|c|c|c|c|c|c|c|}
\hline & \multicolumn{5}{|c|}{ Birth weight (g) } & \multicolumn{2}{|c|}{$P$ for trend ${ }^{1}$} \\
\hline & $\begin{array}{l}<2500 \\
(n=16)\end{array}$ & $\begin{array}{c}2500-2999 \\
(n=49)\end{array}$ & $\begin{array}{c}3000-3499 \\
(n=109)\end{array}$ & $\begin{array}{c}3500-3999 \\
(n=63)\end{array}$ & $\begin{array}{l}\geq 4000 \\
(n=22)\end{array}$ & unadjusted & adjusted $^{2}$ \\
\hline Weight (kg) & 24.7 & 24.1 & 24.5 & 26.3 & 28.4 & $<0.001$ & - \\
\hline Height $(\mathrm{cm})$ & 125.9 & 125.3 & 126.0 & 128.7 & 132.0 & $<0.001$ & $<0.001$ \\
\hline BMI $\left(\mathrm{kg} / \mathrm{m}^{2}\right)$ & 15.5 & 15.3 & 15.4 & 15.9 & 16.2 & 0.012 & $0.003^{3}$ \\
\hline Sum four skinfolds (mm) & 28.8 & 27.8 & 26.5 & 28.5 & 26.1 & NS & $<0.001^{3}$ \\
\hline Body fat $(\%)$ & 20.0 & 19.1 & 18.4 & 19.0 & 17.9 & NS & $<0.001^{3}$ \\
\hline Fat mass (kg) & 5.1 & 4.8 & 4.6 & 5.2 & 5.2 & NS & $<0.001^{3}$ \\
\hline Fat-free mass ( $\mathrm{kg}$ ) & 19.6 & 19.3 & 19.9 & 21.1 & 23.2 & $<0.001$ & $<0.001$ \\
\hline Leptin $(\mu \mathrm{g} / \mathrm{L})$ & 5.1 & 4.0 & 3.5 & 3.8 & 3.1 & NS & $<0.001^{3}$ \\
\hline Glucose $(\mathrm{mmol} / \mathrm{L})$ & 4.8 & 4.6 & 4.7 & 4.7 & 4.7 & NS & NS \\
\hline Insulin (pmol/L) & 48.3 & 37.7 & 35.5 & 37.5 & 38.2 & NS & $0.006^{3}$ \\
\hline Proinsulin (pmol/L) & 7.4 & 6.7 & 6.7 & 7.2 & 6.6 & NS & NS \\
\hline Proinsulin to insulin ratio & 0.16 & 0.20 & 0.22 & 0.21 & 0.22 & NS & 0.017 \\
\hline Insulin resistance (HOMA) & 1.4 & 1.1 & 1.0 & 1.1 & 1.1 & NS & $0.009^{3}$ \\
\hline Beta-cell function (HOMA) & 108.1 & 96.9 & 86.4 & 86.1 & 94.4 & 0.031 & $0.010^{3}$ \\
\hline Total cholesterol (mmol/L) & 4.10 & 4.04 & 4.10 & 4.09 & 3.88 & NS & NS \\
\hline LDL-cholesterol $(\mathrm{mmol} / \mathrm{L})$ & 2.47 & 2.49 & 2.47 & 2.50 & 2.23 & NS & NS \\
\hline HDL-cholesterol ( $\mathrm{mmol} / \mathrm{L})$ & 1.39 & 1.29 & 1.39 & 1.32 & 1.42 & NS & NS \\
\hline Apolipoprotein Al (mg/L) & 1376 & 1331 & 1376 & 1331 & 1399 & NS & NS \\
\hline Apolipoprotein B (mg/L) & 750 & 749 & 733 & 740 & 650 & NS & NS \\
\hline Triacylglycerol $(\mathrm{mmol} / \mathrm{L})$ & 0.54 & 0.58 & 0.54 & 0.58 & 0.49 & NS & NS \\
\hline Lipoprotein (a) $(\mathrm{mg} / \mathrm{L})^{4}$ & 67 & 92 & 73 & 73 & 39 & NS & NS \\
\hline
\end{tabular}

BMI, body mass index; HOMA, homeostasis model assessment; NS, not significant.

' $P$ values are derived from simple (unadjusted) and multiple (adjusted) linear regression using continuous variables.

${ }^{2}$ Adjusted for length of gestation, sex, age at follow-up, and current body mass.

${ }^{3}$ For negative association with birth weight.

${ }^{4}$ Values are geometric means. 


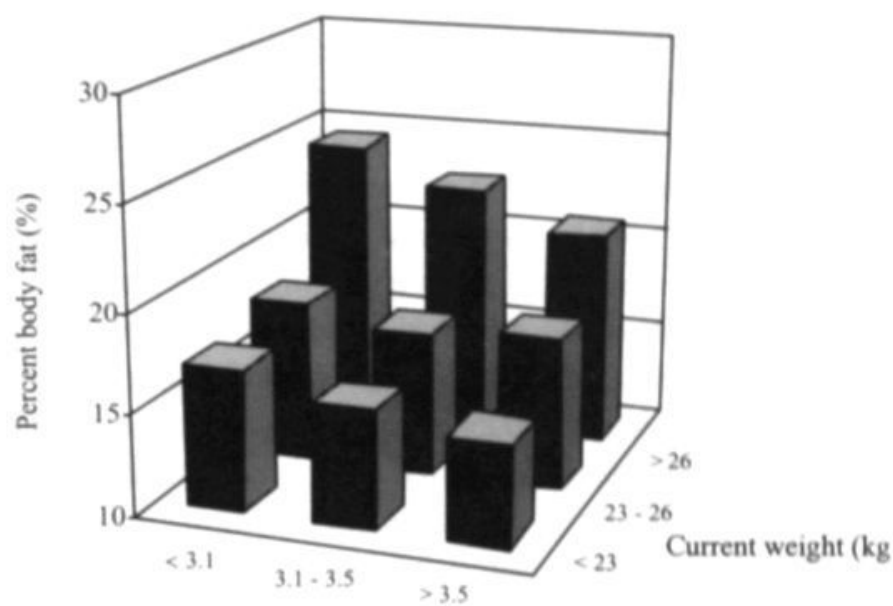

Birth weight $(\mathrm{kg})$

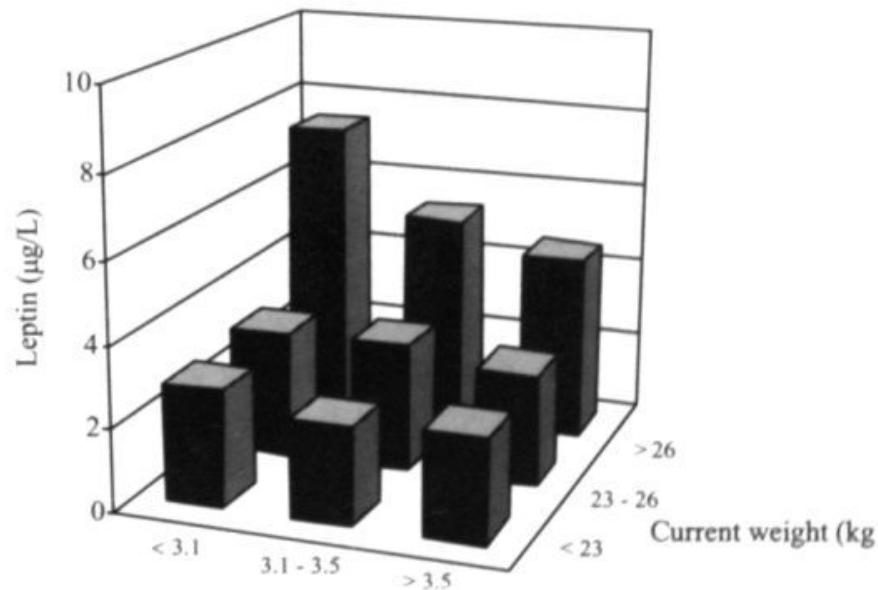

Birth weight $(\mathrm{kg})$

Figure 7.1: Percent body fat and plasma leptin concentration in 7-year-old children according to tertiles of birth weight and current weight $(n=259)$. Children with a lower weight at birth had a higher body fatness and plasma leptin concentration, especially in children with a high current weight. $P<0,001$ for the associations of body fatness and plasma leptin with birth weight and current weight (adjusted for length of gestation, age, and sex by multiple regression analysis). 


\section{Infant nutrition}

It is generally accepted that breast-feeding has important beneficial effects on the wellbeing of infants and children. In relation to later cardiovascular risk, however, the effect of breastfeeding is less clear. Both favorable and adverse influences on cardiovascular risk have been attributed to breastfeeding. Ravelli et al. ${ }^{38}$ report higher mean LDL-cholesterol and lower mean HDL-cholesterol concentrations, a lower LDL/HDL ratio, and higher mean glucose concentrations after an oral glucose tolerance test in bottle-fed individuals when compared to exclusively breast-fed adults. The incidence of Type II diabetes mellitus is lower among Pima Indians who were breastfed during infancy. ${ }^{39,40}$ Additionally, the prevalence of obesity is lower in breast-fed children. $^{41,42}$ A clear dose-dependent effect of breastfeeding on the prevalence of childhood obesity was observed during some studies, ${ }^{41,43}$ but not during others. ${ }^{44}$ On the other hand, Leeson and colleagues ${ }^{45}$ reported that breastfeeding is associated with a lower arterial distensibility in early adult life. In addition, the lowest prevalence of obesity in 6-year-old children was found in association with the shortest period of breastfeeding in another study. ${ }^{46}$ In the same study, the risk of overweight or obesity was greatest for those children who either never received breast-milk or those breastfed for the longest period of time. ${ }^{46}$

The in Chapter $4 \& 5$ reported cardiovascular risk indicator levels did not differ between breast-fed children and formula-fed children (not shown). In addition, the phospholipid $\gamma$-linolenic acid and dihomo- $\gamma$-linolenic acid concentrations, of umbilical cord blood and of blood obtained at follow-up, did not differ between breast-fed children and formula-fed children. Interestingly, infant feeding mode did seem to have an influence on the associations found between triacylglycerol and the phospholipid concentrations of these fatty acids (Figure 7.2). The negative relation with $\gamma$-linolenic acid and dihomo- $\gamma$-linolenic acid measured at birth was more pronounced in children who were breast-fed during infancy. Whereas, the positive relation with $\gamma$-linolenic acid and dihomo- $\gamma$-linolenic acid measured at seven years of age was more pronounced in children who were formula-fed during infancy. However, the interaction terms of the multiple regression models did not reach statistical significance.

In contrast to breast milk, most of the commercially available formulas in the Netherlands do not contain $\gamma$-linolenic acid or dihomo- $\gamma$-linolenic acid. ${ }^{47}$ Breast-fed children, therefore, have a higher $\gamma$-linolenic acid and dihomo- $\gamma$-linolenic acid availability during infancy than formula-fed children. It is tempting to conclude that this could explain observed differences between breast-fed children and formula-fed children. However, the involvement of other factors that differ between breast-fed children and formula-fed children can not be excluded. The mother's decision to breastfeed her child may depend on factors that could also have an influence on the cardiovascular risk profile of her offspring. For instance, maternal obesity has been shown to interfere with the initiation and duration of breastfeeding. ${ }^{48,49}$ Mothers of heavier babies may switch to bottle-feeding earlier than mothers of smaller infants 
because of a different energy intake and suckling behavior of her child. ${ }^{48,50}$ The observed differences between breast-fed children and formula-fed children may also be accounted for by associated differences in social factors. In the present study, the information on infant nutrition was obtained seven years after birth. The results might have been biased by this fact (i.e. recall bias).

\section{Limitations of the study}

\section{Study design}

The study described in this thesis is in essence a non-concurrent prospective study (also called a historical cohort study or a retrospective cohort study). ${ }^{51}$ Historical data (essential fatty acid concentrations measured in umbilical cord blood shortly after birth) were related to prospectively measured outcome variables (cardiovascular risk indicators measured at age seven). The absence of a random allocation of the studied subjects is critical in the interpretation of the results obtained through a cohort study. ${ }^{51,52}$ The associations found may indicate causal relationships but the strength of evidence is less than when obtained by a well-designed randomized trial. Children with different levels of exposure for a particular fatty acid may also differ with respect to other (unknown) factors. The guarantee that children with different fatty acid concentrations at birth are otherwise comparable is namely lost by the absence of a random allocation.

Although the associations found did not attenuate after correction for several potential confounding factors, residual confounding can not be excluded. Thus, inherent to the design of our study we are left with the uncertainty that confounding factors could have caused the observed associations. Non-participation and non-response can introduce major biases that can complicate the interpretation of findings in cohort studies. ${ }^{51}$ If children are selectively lost to follow-up, the associations found may be the result of non-participation of a particular group of children. As shown in Chapter 1 (Figure 1.3), only about $40 \%$ of the eligible children participated. The birth characteristics of these children, however, were not significantly different from the children who did not attend our clinic (Table 1.1). Moreover, the concentrations of $\gamma$ linolenic acid and dihomo- $\gamma$-linolenic acid in umbilical cord blood did not differ between participants and non-participants. Mean \pm SD concentrations for participants and non-participants were: $0,05 \pm 0,04 \% \mathrm{wt} / \mathrm{wt}$ and $0,05 \pm 0,04 \% \mathrm{wt} / \mathrm{wt}$ for $\gamma$-linolenic acid and 5,15 $\pm 0,88 \% \mathrm{wt} / \mathrm{wt}$ and $5,06 \pm 0,87 \% \mathrm{wt} / \mathrm{wt}$ for dihomo- $\gamma$-linolenic acid, respectively. These findings all seem to indicate that the investigated children comprised a representative sample of the entire cohort. Nevertheless, outcome variables could not be compared between participants and non-participants, and a residual bias due to a selective loss to follow-up can not be fully excluded.

We included all children for whom fatty acid concentrations at birth were known. This permitted the study of several candidate fatty acids in relation to the 
outcome variables, which was an important advantage considering the explorative nature of our study. The risk of this approach is that due to multiple testing of associations some relations will appear statistically significant purely due to chance. The importance of such change findings may subsequently be overestimated.

At birth

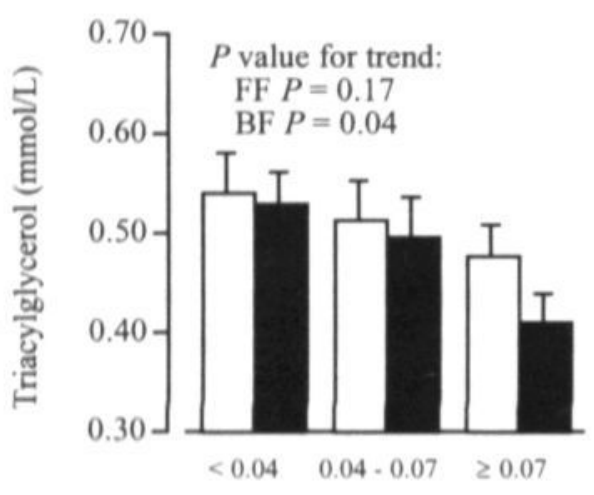

At seven years

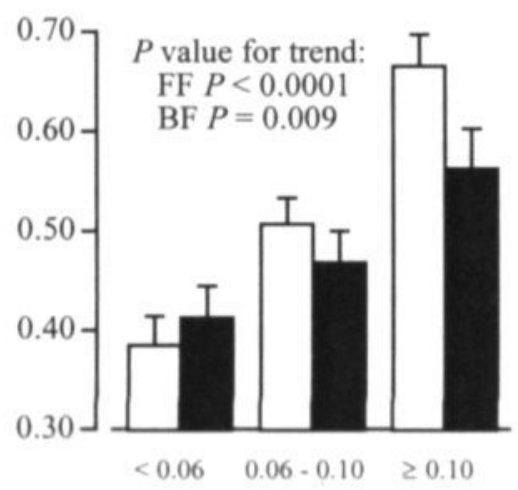

Tertiles of $18: 3 n-6(\% w t / w t)$
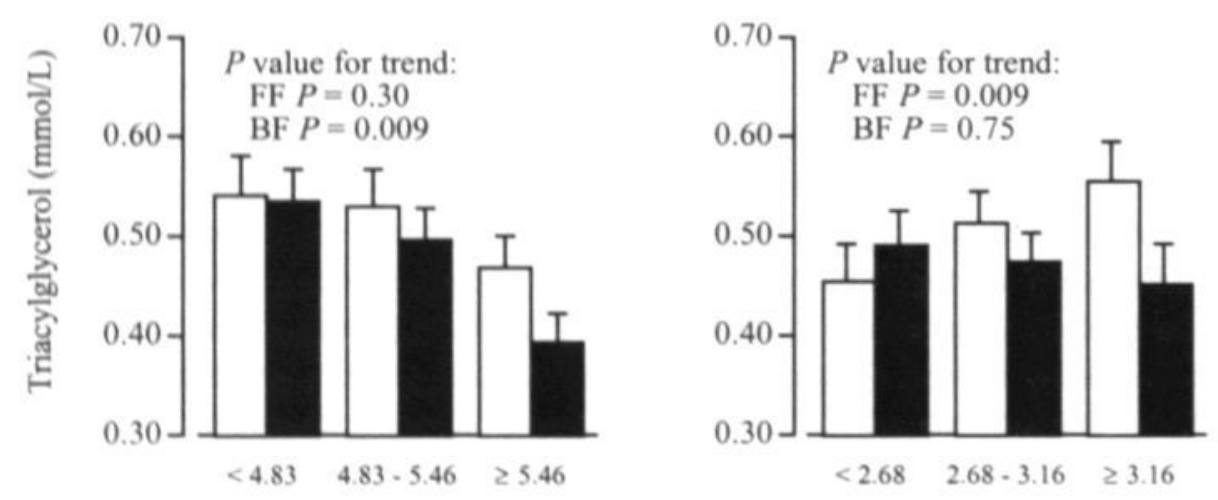

Tertiles of $20: 3 n-6(\% \mathrm{wt} / \mathrm{wt})$

Formula-fed (FF)

Breast-fed (BF)

Figure 7.2: Relations between fasting plasma triacylglycerol concentrations in 7-year-old children and plasma phospholipid $\gamma$-linolenic acid and dihomo- $\gamma$-linolenic acid concentrations in umbilical cord blood or in blood obtained at seven years of age according to infant feeding mode. Values are geometric means \pm SE. $P$ values are from linear regression models with log-triacylglycerol level as dependent variable and either $\gamma$-linolenic acid or dihomo- $\gamma$-linolenic acid concentration as the independent variable. 
Table 7.2: Multiple regression analyses of log-transformed insulin concentration ( $\mathrm{pmol} / \mathrm{L}$ ) in sevenyear-old children with plasma phospholipid $\gamma$-linolenic acid concentrations, adjusted for the arachidonic acid to linoleic acid ratio.

\begin{tabular}{lcr}
\hline & Coefficient $^{\prime}(95 \% \mathrm{CI})$ & $P$ \\
\hline Model 1 & $-6.6(-24.2 ; 11.0)$ & 0.460 \\
$\quad$ Arachidonic acid to linoleic acid ratio & & \\
Model 2 & $-5.7(-9.6 ;-1.9)$ & 0.004 \\
$\gamma$-Linolenic acid at birth $(\% \circ \mathrm{wt} / \mathrm{wt})$ & $5.7(3.2 ; 8.1)$ & $<0.001$ \\
$\gamma$-Linolenic acid at seven years $(\% \circ \mathrm{wt} / \mathrm{wt})$ & $-9.9(-26.7 ; 6.9)$ & 0.247 \\
\hline Arachidonic acid to linoleic acid ratio & &
\end{tabular}

${ }^{1}$ Coefficients are percentage changes in log-insulin concentration per unit change in predictor variables (Note that one unit change in $\gamma$-linolenic acid concentration equals $0.1 \% \mathrm{wt} / \mathrm{wt}$ ).

The fact that $\gamma$-linolenic acid concentration at birth related to several outcome variables (triacylglycerol, insulin, proinsulin, leptin, and body fatness), which are all components of the same so-called insulin resistance syndrome, and the fact that adjustments for potential confounding factors did not attenuate these associations, both seem to indicate that the associations found are more than just change findings.

The observed associations between $\gamma$-linolenic acid concentrations at birth and at seven years of age on one hand and the insulin resistance variables on the other hand could result from a 'mechanism' of reverse causality. Insulin has a positive influence on the activity of the enzyme delta-6-desaturase. ${ }^{53}$ Delta-6-desaturase stimulates the conversion of linoleic acid (18:2n-6) into $\gamma$-linolenic acid (Figure 1.2). A higher plasma concentration of insulin could thus explain a higher $\gamma$-linolenic acid content of plasma lipid fractions. Conversely, low levels of circulating insulin could relate to lower $\gamma$ linolenic acid concentrations. Other factors with an influence on insulin metabolism (including genetic factors) could differ between infants with different $\gamma$-linolenic acid concentrations at birth and explain our observations. Our data may thus also fit the "fetal insulin hypothesis", which has been proposed as an alternative explanation for the associations between birth weight and adult cardiovascular diseases. ${ }^{54}$ Interestingly, we observed an interaction between birth weight and a positive family history of Type II diabetes in relation to childhood insulin concentrations. Birth weight was negatively related to insulin concentrations only in children with a positive family history of diabetes (Chapter 4). Recently, common gene variants, that are associated with adult impaired glucose tolerance and Type II diabetes mellitus, have also been related to a low weight or ponderal index at birth. ${ }^{55,56}$ It could be of interest to study such polymorphisms in relation to $\gamma$-linolenic acid concentrations at birth.

In an attempt to test the hypothesis of reverse causality, the relation between $\gamma$ linolenic acid and insulin concentration was studied correcting for the arachidonic acid to linoleic acid ratio at age seven. Since delta-6-desaturase is considered to be the ratelimiting enzyme in the conversion of linoleic acid into arachidonic acid, this ratio indirectly reflects delta-6-desaturase activity. No statistically significant relation 
between this ratio and insulin concentration was found. Further, the observed associations between insulin concentration and the $\gamma$-linolenic acid content of umbilical cord blood and blood obtained at seven years of age were not attenuated when corrected for the arachidonic acid to linoleic acid ratio (Table 7.2). The arachidonic acid to linoleic acid ratio determined at birth was not related to the insulin concentrations at age seven either $(P=0.21)$. These observations do not support the idea of reverse causality. A small fraction of umbilical cord plasma is still available for some of the children in our cohort. Additional clues might be found by studying insulin concentrations in these samples. If a positive relation between umbilical plasma insulin levels and $\gamma$-linolenic acid concentration is not found, such a mechanism of reverse causality seems less likely.

\section{Use of plasma phospholipid fatty acid concentrations}

In the studies described in Chapters 3, 4 and 5, we used essential fatty acid concentrations (i.e. expressed as \% by wt of total fatty acids) measured in the umbilical cord plasma phospholipid fraction as a measure for the availability of these fatty acids during fetal life. Fatty acid concentrations in maternal plasma lipids measured during pregnancy are known to be strong predictors of fatty acid concentrations in umbilical plasma (Chapter 2) ${ }^{23,57}$ Both, maternal and umbilical fatty acid concentrations, are influenced by the maternal dietary intake of fatty acids (Table 2.6) ${ }^{58-62}$ Additionally, umbilical fatty acid concentrations measured after delivery, as in the present study, are essentially similar to concentrations found in fetal plasma when measured at a comparable gestational age.$^{63}$ For these reasons, we assume that umbilical cord blood fatty acid concentrations are a reasonable reflection of the intrauterine fatty acid supply. The turnover of fatty acids in plasma phospholipids is, however, relatively fast. Therefore, fatty acid concentrations in umbilical plasma phospholipids probably only reflect the availability of essential fatty acids during late gestation. This may be of relevance since exposure to the Dutch famine during early gestation, rather than during late gestation, has been associated to adult plasma cholesterol and lipoprotein concentrations. ${ }^{64}$ It could explain why we did not find a relation between umbilical plasma fatty acid concentrations and childhood cholesterol and lipoprotein levels. Other results might have been obtained when erythrocyte membrane or umbilical vascular wall tissue fatty acid concentrations were used as a proxy for essential fatty acid availability during fetal life. Since these data are available for a subset of our study population, such a study could easily be performed in the near future.

Fatty acid concentrations can be expressed as an absolute concentration (in $\mathrm{mg} / \mathrm{L}$ ) or as a relative concentration (\% by wt of total fatty acids), as in Chapter 2 . The use of each expression has its own particular limitations. Absolute concentrations are more dependent on differences in analytical procedures such as the time-point at which the internal standard is added to the samples. ${ }^{65}$ When compared to relative concentrations, the comparability of absolute concentrations between laboratories is 
smaller, and the measurement errors are, usually, larger. Another important limitation of absolute concentrations is their dependence on the total lipid pool size. The absolute concentration of individual fatty acids will be higher in a subject with a higher total amount of phospholipids in his blood. For this reason, absolute concentrations of individual fatty acids can not be used for the study of associations with cardiovascular risk indicators that could relate to the total phospholipid pool size (like plasma lipid concentrations).

As shown in Figure 7.3, the total amount of phospholipid associated fatty acids (which reflects the phospholipid pool size) is strongly related to plasma cholesterol concentration. For these reasons, relative concentrations were used during the studies described in Chapters 3, 4 and 5. The use of relative concentrations, however, has important limitations as well. ${ }^{66}$ The individual fatty acid concentrations measured are not obtained from independent observations, this is especially true for relative concentrations of the more abundant fatty acids. An increase in one proportion relates with a decrease in some other proportion, because the sum of all fatty acids is $100 \%$. As a result, associations between individual fatty acid concentrations can be found. This problem of interdependency between fatty acid concentrations is not unique to relative fatty acid concentrations; a similar problem would arise when absolute concentrations are used. Since the amount of phospholipid associated fatty acids might be higher in one person compared to another because of a larger phospholipid pool, positive relations between absolute fatty acid concentrations can just be found as a result of the differences in phospholipid pool sizes. These methodological problems, inherent to studies in which fatty acid concentrations are related to one another, are hard to overcome. The artificial effects created are superimposed on the true biological relations that could be present. Therefore, a careful interpretation is needed when studying relations between individual fatty acid concentrations measured in the same matrix.

\section{Use of cardiovascular risk indicators as outcome variables}

Major cardiovascular events are rare in children. Therefore, the risk for the development of cardiovascular diseases had to be studied indirectly, using cardiovascular risk indicators. Evidence from autopsy studies indicate that the development of atherosclerosis starts during childhood, and that childhood cardiovascular risk indicator levels are related to the extent of the atherosclerotic lesions present. Moreover, children tend to maintain their ranking order from childhood into adulthood with respect to several cardiovascular risk indicators; a phenomenon referred to as "tracking" (for references see Chapter 1). These findings imply that the evaluation of cardiovascular risk indicators in children is a useful tool in identifying groups of individuals who are at a potential risk for developing cardiovascular diseases later in life. An important question that remains is whether the differences in risk indicator levels found in children are clinically relevant. Individual risk indicator levels 
measured during childhood may not relate to true cardiovascular events in adulthood. Whether fatty steaks seen in arteries of children are related to adult cardiovascular disease is not known. At this point, childhood cardiovascular risk indicators (like childhood cholesterol) have been associated with adult risk indicators (like adult cholesterol), but not with true endpoints like coronary heart disease morbidity or mortality. ${ }^{68}$ Moreover, it is not known whether interventions started early in life, and designed to reduce cardiovascular risk indicator levels in children, are safe and equally effective in the prevention of cardiovascular events than interventions started in adulthood. $^{68}$

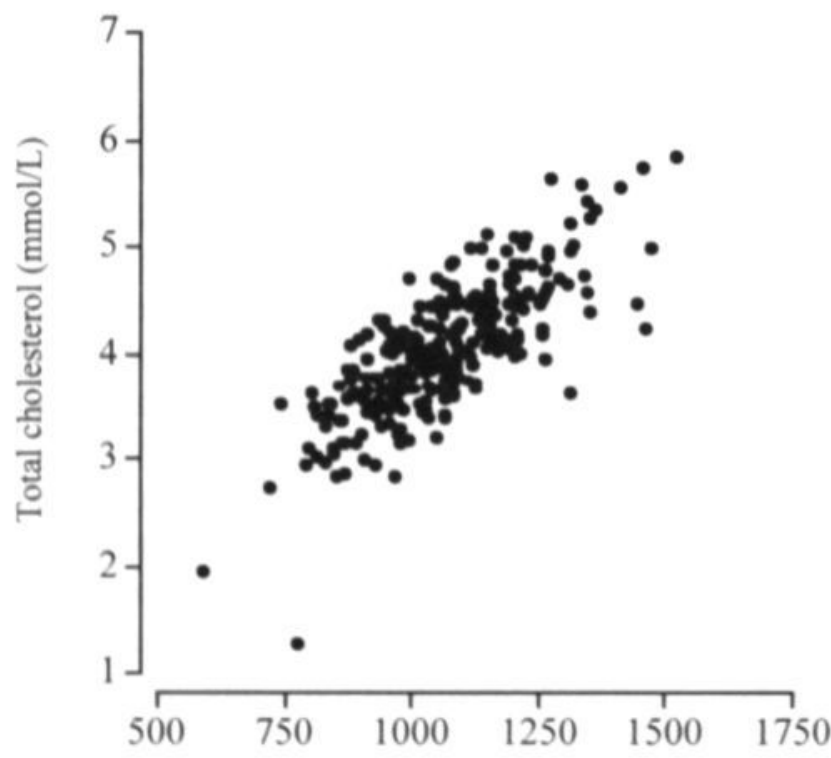

Total fatty acids $(\mathrm{mg} / \mathrm{L})$

Figure 7.3: Relation between plasma total cholesterol concentration and the total fatty acid content of plasma phospholipids in 7-year-old children $(P<0,0001 ; \mathrm{r}=0.75 ; n=259)$.

Many children with higher risk indicator levels will have normal levels in adulthood even in the absence of prescribed individual interventions. ${ }^{67}$ Risk indicator level screening could thus unjustly label children as patients with a "disease", causing unjustified anxiety and stigmatization. Therefore, the measurement of cardiovascular risk indicators in children should not be used as a universal screening tool to identify individuals with an increased risk and to select them for individual interventions, like 
drug therapy. ${ }^{68,69}$ This does not mean that lowering risk indicator levels in children could not have beneficial effects. Based on the indirect evidence available, selective screening and the subsequent treatment of children who are at the highest risk (e.g. children from families with hypercholesterolemia, children with diabetes, or obese children) is recommendable. ${ }^{69,70}$ For other children, a population-wide approach (e.g. improving dietary composition, increasing physical activity, discouraging cigarette smoking, and avoiding excessive weight gain) is considered a more appropriate strategy to lower the risk for cardiovascular diseases later in life. ${ }^{69,70}$ An advantage of the latter approach is that even a relatively small reduction of population-average risk indicator levels in childhood could substantially decrease the incidence of coronary heart disease, when carried into adulthood. ${ }^{69}$ In this light, small differences in risk indicator levels found between groups of children could be clinically relevant indeed.

\section{Critique on studies on the fetal origins hypothesis}

The fetal origins hypothesis is important because of the potential impact on public health it might have. One of the biggest problems, however, is that it seems impossible to provide conclusive evidence. To test the hypothesis, investigators used retrospective studies, based on existing data sets and natural occurring events, like the Dutch Hunger Winter. As a consequence, the associations found could be biased and may not indicate causal relationships. Therefore, it is not astonishing, that the most important points of critique on studies on the fetal origins hypothesis are the possibility of selection bias and confounding, for instance by socioeconomic status. ${ }^{71-73}$ Other major concerns are the inconsistencies reported within and across the studies and the considerable broadening of the hypothesis. ${ }^{74}$ The fetal origins hypothesis extended from cardiovascular diseases and related disorders to stroke, atopy, cancer, osteoporosis, depression, and aging. A more precise expression of the hypothesis tested, and adherence to that expression has therefore been suggested. ${ }^{74}$

When studying the relation between potential cause and subsequent events, which are separated by decades, losses to follow-up are almost inevitable. Indications for the presence of a selection bias can be found, e.g. by comparing participants with non-participants, but a selection bias can never be completely excluded. The use of a high quality data set, and an almost complete follow-up, ${ }^{76}$ may overcome this problem of selection but does not exclude the possibility of confounding. And although recent studies indicate that the association between size at birth and adult cardiovascular risk is not simply explained by differences in socio-economic background, ${ }^{76,77}$ confounding by other unknown factors can not be excluded. However, to conduct randomized trails - which may provide the stronger evidence needed - one would first have to know which intervention might have the desirable effect.

The study described in this thesis was explorative in nature, therefore, the hypothesis was not precisely expressed; a selection bias might have occurred, and 
confounding can not be fully excluded. Thus, many of the above mentioned points of critique are applicable to the study described in this thesis as well. But this does not mean we should simply disparage the results obtained. The shortcomings mentioned not necessarily lead to wrong conclusions. ${ }^{78} \mathrm{~A}$ bias, when present, may be small or irrelevant. Furthermore, the findings of the present study allow many points of critique to be addressed during future studies. A more precise hypothesis can now be generated and tested. Knowing a candidate nutritional factor that could be involved, intervention studies using a random allocation of studied subjects can be conducted, which will avoid many methodological problems and reduce the possibility of confounding. Therefore, despite the reservations discussed above, there seems to be sufficient evidence to justify further scrutiny.

\section{Interpretations and recommendations for future studies}

From the study described in the present thesis it can be concluded that concentrations of essential fatty acids, measured in umbilical cord blood, are related to several cardiovascular risk indicators determined in children at seven years of age. Based on the findings described, it seems that a lower availability of $\gamma$-linolenic acid (and/or its direct elongation product dihomo- $\gamma$-linolenic acid) during fetal life is related to a less favorable risk profile. Increasing the maternal intake of $\gamma$-linolenic acid during pregnancy is expected to enhance the fetal availability of both $\gamma$-linolenic acid and dihomo- $\gamma$-linolenic acid. Our findings therefore suggest that $\gamma$-linolenic acid supplementation in pregnant women may have beneficial effects on the risk profile of her offspring. In addition, some observations indicate that an increased $\gamma$-linolenic acid availability during infancy may have an additive effect (see above). The positive associations found at age seven, on the other hand, seem to suggest that a lower intake of this fatty acid is recommendable later in life. Because of the many limitations mentioned before, our study does not provide sufficient evidence to fully support these hypotheses. As stated above, other mechanisms could explain our findings as well. At this point it would be premature to actually recommend a higher intake of this fatty acid to pregnant women or infants. However, the relations described do indicate that $\gamma$ linolenic acid is an interesting nutrient in light of the fetal origins hypothesis, and thus for further scrutiny.

Ideally, the potential effect and safety of an increased availability of $\gamma$-linolenic acid during fetal life is studied using randomized supplementation trials during pregnancy. As a first step, the efficacy of $\gamma$-linolenic acid supplementation could be tested by short-term animal experiments. Another option could be to recruit children from previous randomized trials. Intervention studies have been performed to analyze the effects of $\gamma$-linolenic acid supplementation on the development of atopic diseases in children. The measurement of cardiovascular risk indicators, especially components of the insulin resistance syndrome, in those children allows comparisons between 
randomized groups within a short time window. New studies are also needed to investigate the effects of $\gamma$-linolenic acid supplementation in pregnant women and infant formula. For a truly prospective test, these studies would need a long follow-up period. Because of the relatively small effects found, the tested population should also be large enough to accurately estimate population-wide benefits. If studies like these confirm that a higher availability of $\gamma$-linolenic acid during fetal life and maybe infancy, relates to a more favorable risk profile later in life, population-wide supplementation of $\gamma$-linolenic acid during pregnancy and infancy could be a relatively simple new tool in the prevention of cardiovascular diseases.

\section{References}

1. Barker DJP. Mothers, babies and health in later life. Second ed. Edinburgh: Churchill Livingstone; 1998.

2. Barker DJP. The fetal and infant origins of disease. Eur J Clin Invest. 1995;25:457-463.

3. Lucas A. Programming by early nutrition in man. In: Bock GR, Whelan J, eds. The childhood environment and adult disease. Chichester: John Wiley and Sons; 1991:38-55.

4. O'Brien PMS, Wheeler T, Barker DJP, eds. Fetal programming: influences on development and diseases in later life. London: RCOG Press; 1999.

5. Olson SF. Further on the association between retarded foetal growth and adult cardiovascular disease. Could low intake of marine diets be a common cause? J Clin Epidemiol. 1994;47:565569 .

6. James WPT. Long-term fetal programming of body composition and longevity. Nutr Rev. 1997;55:S31-S43.

7. Sattar N, Berry C, Greer IA. Essential fatty acids in relation to pregnancy complications and fetal development. Br J Obstet Gynaecol. 1998;105:1248-1255.

8. Crawford MA, Doyle W, Drury P, Lennon A, Costeloe K, Leighfield M. n-3 and n-6 fatty acids during early human development. J Intern Med. 1989;225:159-169.

9. Koletzko B, Braun M. Arachidonic acid and early human growth: is there a relation? Ann Nutr Metab. 1991;35:128-131.

10. Leaf AA, Leighfield MJ, Costeloe KL, Crawford MA. Long chain polyunsaturated fatty acids and fetal growth. Early Hum Dev. 1992;30:183-191.

11. Felton CV, Chang TC, Crook D, Marsh M, Robson SC, Spencer JAD. Umbilical vessel wall fatty acids after normal and retarded fetal growth. Arch Dis Child. 1994;70:F36-F39.

12. Foreman-van Drongelen MMHP, Houwelingen ACv, Kester ADM, Hasaart THM, Blanco CE, Hornstra G. Long-chain polyunsaturated fatty acids in pre-term infants: status at birth and its influence on postnatal levels. J Pediatr. 1995;126:611-618.

13. Woltil HA, Beusekom CMv, Schaafsma A, Muskiet FAJ, Okken A. Long-chain polyunsaturated fatty acid status and early growth of low birth weight infants. Eur J Pediatr. 1998; 157:146-152.

14. Carlson SE, Cooke RJ, Werkman SH, Tolley EA. First year growth of preterm infants fed standard compared to marine oil supplemented formula. Lipids. 1992;27:901-907.

15. Vilbergsson G, Samsioe G, Wennergren M, Karlsson K. Essential fatty acids in pregnancies complicated by intrauterine growth retardation. Int J Gyneacol Obstet. 1991;36:277-286.

16. Percy P, Vilbergsson G, Percy A, Mansson J, Wennergren M, Svennerholm L. The fatty acid composition of placenta in intrauterine growth retardation. Biochim Biophys Acta. 1991;1084:173-177. 
17. Farquharson J, Jamieson EC, Logan RW, McFadyn MB, Patrick WJA, Howatson AG, Cockburn F. Infant growth and aorta total lipid fatty acids. Arch Dis Child. 1998; 79:28-32.

18. Carlson SE, Werkman SH, Peeples JM, Cooke RJ, Tolley EA. Arachidonic acid status correlates with first year growth in preterm infants. Proc Natl Acad Sci USA. 1993;90:10731077.

19. Auested N, Montalto MB, Hall RT, Fitzgerald KM, Wheeler RE, Conner WE, Neuringer M, Conner SL, Taylor JA, Hartmann EE. Visual acuity, erythrocyte fatty acid composition, and growth in term infants fed formulas with long chain polyunsaturated fatty acids for one year. Pediatr Res. 1997;41:1-10.

20. Birch EE, Hoffman DR, Uauy R, Birch DG, Prestidge C. Visual acuity and the essentiality of docosahexaenoic acid and arachidonic acid in the diet of term infants. Pediatr Res. 1998;44:201-209.

21. Makrides M, Neumann MA, Simmer K, Gibson RA. Dietary long-chain polyunsaturated fatty acids do not influence growth of term infants: a randomized clinical trial. Pediatrics. 1999;104:468-475.

22. Lucas A, Stafford M, Morley R, Abbott R, Stephenson T, MacFadeyen U, Elias-Jones A, Clements H. Efficacy and safety of long-chain polyunsaturated fatty acid supplementation of infant-formula milk : a randomised trail. Lancet. 1999;354:1948-54.

23. Al MD, Houwelingen $\mathrm{ACv}$, Kester $\mathrm{AD}$, Hasaart $\mathrm{TH}$, de Jong $\mathrm{AE}$, Hornstra G. Maternal essential fatty acid patterns during normal pregnancy and their relationship to the neonatal essential fatty acid status. Br J Nutr. 1995;74:55-68.

24. Barker DJP, Hales CN, Fall CHD, Osmond C, Phillips K, Clark PMS. Type 2 (non-insulindependent) diabetes mellitus, hypertension, and hyperlipidaemia (syndrome $\mathrm{X}$ ): relation to reduced fetal growth. Diabetologia. 1993;36:62-67.

25. Hales CN, Barker DJP, Clark PMS, Cox LJ, Fall C, Osmond C, Winter PD. Fetal and infant growth and impared glucose tolerance at age 64. BMJ. 1991;303:1019-1022.

26. Lithell HO, McKeigue PM, Berglund L, Mohson R, Lithell UB, Leon DA. Relation of size at birth to non-insulin-dependent diabetes and insulin concentrations in men aged 50-60 years. BMJ. 1996;312:406-410.

27. Curhan GC, Willett WC, Rimm EB, Spiegelman D, Ascherio AL, Stampfer MJ. Birth weight and adult hypertension, diabetes mellitus, and obesity in US men. Circulation. 1996;94:32463250.

28. Law CM, Gordon GS, Shiell AW, Barker DJP, Hales CN. Thinness at birth and glucose tolerance in seven-year-old children. Diabet Med. 1995;12:24-29.

29. Bavdekar A, Yajnik CS, Fall CHD, Bapat S, Pandit AN, Deshpande V, Bhave S, Kellingray SD, Joglekar C. Insulin resistance syndrome in 8-year-old Indian children. Diabetes. 1999:48:2422-2429.

30. Hediger ML, Overpeck MD, Kuczmarski RJ, McGlynn A, Maurer KR, Davis WW. Muscularity and fatness of infants and young children born small-for-gestational-age. Pediatrics. 1998;102:e60.

31. Ravelli ACJ, Meulen JHPvd, Osmond C, Barker DJP, Bleker OP. Glucose tolerance in adults after in utero exposure to the Dutch Famine. Lancet. 1998;351:173-177.

32. Howard BV, Gidding SS, Liu K. Association of apolipoprotein E phenotype with plasma lipoproteins in African-American and white young adults. The CARDIA Study. Am J Epidemiol. 1998;148:859-868.

33. Smit M, Knijff Pd, Rosseneu M, Bury J, Klasen E, Frants R, Havekes LM. Apolipoprotein E polymorphism in the Netherlands and its effects on plasma lipid and apolipoprotein levels. Hum Genet. 1988;80:287-292. 
34. Sanghera DK, Ferrell RE, Aston CE, McAllister AE, Kamboh MI, Kimm SYS. Quantitative effects of the apolipoprotein E polymorphism in a biracial sample of 9-10-year-old girls. Atherosclerosis. 1996;126:35-42.

35. Kondo I, Berg K, Drayna D, Lawn R. DNA polymorphism at the locus for human cholesteryl ester transfer protein (CETP) is associated with high density lipoprotein cholesterol and apolipoprotein levels. Clin Genet. 1989;35:49-56.

36. Kuivenhoven JA, Knijf Pd, Boer JMA, Smalheer HA, Botma GJ, Seidell JC, Kastelein JJP, Pritchard PH. Heterogeneity at the CETP gene locus. Influence on plasma CETP concentrations and HDL cholesterol levels. Aterioscler Thromb Vasc Biol. 1997;17:560-568.

37. Gudnason V, Kakko S, Nicaud V, Savolainen MJ, Kesăniemi YA, Tahvanainen E, Humphries S. Cholesteryl ester transfer protein gene effect on CETP activity and plasma high-density lipoprotein in European populations. Eur J Clin Invest. 1999;29:116-128.

38. Ravelli AC, Meulen JHvd, Osmond C, Barker DJ, Bleker OP. Infant feeding and adult glucose tolerance, lipid profile, blood pressure, and obesity. Arch Dis Child. 2000;82:248-252.

39. Pettit DJ, Forman MR, Hanson RL, Knowler WC, Bennett PH. Breast feeding and incidence of non-insulin-dependent diabetes mellitus in Pima Indians. Lancet. 1997;350:166-168.

40. Pettitt DJ, Knowler WC. Long-term effects of the intrauterine environment, birth weight, and breast-feeding in Pima Indians. Diabetes Care. 1998;21:B138-141.

41. Kries Rv, Koletzko B, Sauerwald T, Mutius Ev, Barnert D, Grunert V, Voss Hv. Breast feeding and obesity: cross sectional study. BMJ. 1999;319:147-150.

42. Kries Rv, Koletzko B, Sauerwald T, Mutius Ev. Does breast feeding protect against childhood obesity? Adv Exp Med Biol. 2000;478:29-39.

43. Liese AD, Hirsch T, Mutius Ev, Keil U, Leupold W, Weiland SK. Inverse association of overweight and breast feeding in 9 to 10-year-old children. Int $J$ Obes Relat Metab Disord. 2001;25:1644-1650.

44. Hediger ML, Overpeck MD, Kuczmarski RJ, Ruan WJ. Association between infant breastfeeding and overweight in young children. JAMA. 2001;285:2453-2460.

45. Leeson CP, Kattenhorn M, Deanfield JE, Lucas A. Duration of breast feeding and arterial distensibility in early adult life: population based study. BMJ. 2001;322:643-647.

46. Wadsworth M. Breast feeding and obesity. Relation may be accounted for by social factors [Letter]. BMJ. 1999;319:1576.

47. Huisman M, Beusekom CMv, Lanting CI, Nijboer HJ, Muskiet FAJ, Boersma ER. Triglycerides, fatty acids, sterols, mono- and disaccharides, and sugar alcohols in human milk and current types of infant formula milk. Eur J Clin Nutr. 1996;50:255-260.

48. Hebebrand J. Breast feeding and obesity. Prolonging breast feeding to reduce obesity may be a burden [Letter]. BMJ. 1999;319:1576.

49. Hilson JA, Rasmussen KM, Kjolhede CL. Maternal obesity and breast-feeding sucsess in a rural population of white women. Am J Clin Nutr. 1997;66:1371-1378.

50. Stunkard AJ, Berkowitz RI, Stallings VA, Schoeller DA. Energy intake, not energy output, is a determinant of body size in infants. Am J Clin Nutr. 1999;69:524-530.

51. Gordis L. Cohort studies. In: Epidemiology. Philadelphia: Saunders; 1996:116-123.

52. Sackett DL, Haynes RB, Guyatt GH, Tugwell P. Clinical epidemiology: a basic science for clinical medicine. Second ed. Boston: Little, Brown and Company; 1991.

53. Brenner RR. Nutritional and hormonal factors influencing desaturation of essential fatty acids. Prog Lipid Res. 1981;20:41-47.

54. Hattersley AT, Tooke JE. The fetal insulin hypothesis: an alternative explanation of the association of low birth weight with diabetes and vascular disease. Lancet. 1999;353:17891792.

55. Casteels K, Ong K, Phillips D, Bendall H, Pembrey M, Poulton J, Dunger D. Mitochondrial 16189 variant, thinness at birth, and type-2 diabetes. Lancet. 1999;353:1499-1500. 
56. Dunger DB, Ong KKL, Huxtable SJ, Sherriff A, Woods KA, Ahmed ML, Golding J, Pembrey ME, Ring S, Bennet ST, Todd JA. Association of the INS VNTR with size at birth. Nature Genet. 1998;19:98-100.

57. Al MD, Hornstra G, Schouw YTvd, Bulstra-Ramakers MT, Huisjes HJ. Biochemical EFA status of mothers and their neonates after normal pregnancy. Early Hum Dev. 1990;24:239-48.

58. Al MD, Badart-Smook A, Houwelingen ACv, Hasaart TH, Hornstra G. Fat intake of women during normal pregnancy: relationship with maternal and neonatal essential fatty acid status. $J$ Am Coll Nutr. 1996;15:49-55.

59. Houwelingen ACv, Dalby-Sorensen J, Hornstra G, Simonis MMG, Boris J, Olson S, Secher NJ. Essential fatty acid status of mothers and their babies after fish oil supplementation during pregnancy. Br J Nutr. 1995;74:723-731.

60. Al MD, Houwelingen ACv, Badart-Smook A, Hornstra G. Some aspects of neonatal essential fatty acid status are altered by linoleic acid supplementation of women during pregnancy. $J$ Nutr. 1995;125:2822-30.

61. Bjerve KS, Brubakk AM, Fougner KJ, Johnson H, Midthjell K, Vik T. Omega-3 fatty acids: essential fatty acids with important biological effects, and serum phospholipid fatty acids as markers of dietary $\omega 3$-fatty acid intake. Am J Clin Nutr. 1993;57:801S-806S.

62. Sanjurjo P, Matorras R, Ingunza N, Rodrigeuz-Alarcón J, Perteagudo L. Blue fish intake and percentual levels of polyunsaturated fatty acids at labor in the mother and newborn infant. Perinat Med. 1994;22:337-344.

63. Houwelingen ACv, Foreman-van Drongelen MMHP, Nicolini U, Nicolaides KH, Al MDM, Kester ADM, Hornstra G. Essential fatty acid status of fetal plasma phospholipids: similar to postnatal values obtained at comparable gestational ages. Early Hum Dev. 1996;46:141-152.

64. Roseboom TJ, Meulen JHPvd, Osmond C, Barker DJP, Ravelli ACJ, Bleker OP. Plasma lipid profile in adults after prenatal exposure to famine. Am J Clin Nutr. 2000;72:1101-1106.

65. Foreman-van Drongelen MMHP, Houwelingen ACv, Kester ADM, Jong AEPd, Blanco CE, Hasaart THM, Hornstra G. Long-chain polyene status of preterm infants with regard to the fatty acid composition of their diet: comparison between absolute and relative fatty acid amounts in plasma and red blood cell phospholipids. Br J Nutr. 1995;73:405-422.

66. Rump P, Hornstra G. Relation between arachidonic acid and docosahexaenoic acid in maternal and neonatal blood. Eur J Clin Nutr. 2001;55:916-917.

67. Lauer RM, Clark WR. Use of cholesterol measurements in childhood for the prediction of adult hypercholesteroleamia: The Muscatine Study. JAMA. 1990;264:3034-3038.

68. Newman TB, Browner WS, Hulley SB. The case against childhood cholesterol screening. JAMA. 1990;264:3039-3043.

69. National Cholesterol Education Program. Highlights of the Report of the Expert Panel on Blood Cholesterol Levels in Children and Adolescents. Am Fam Physician. 1992;45:2127. 2136.

70. International Taskforce for Prevention of Coronary Heart Disease. Prevention of coronary heart disease - scientific background and clinical guidelines 1992. Recommendations of the European Atherosclerosis Society. London: Current Medical Literature Ltd; 1993.

71. Baker D, Illsley R, Vagero D. Today or in the past? The origins of ischeamic heart disease. $J$ Publ Health Med. 1993;15:243-248.

72. Kramer MS, Joseph KS. Enigma of fetal/infant-origins hypothesis [Commentary]. Lancet. 1996:348:1254-1255.

73. Jones SE, Nyengaard JR. Low birth weight and cardiovascular disease: myth or reality? Curr Opin Lipidol. 1998;9:309-312.

74. An overstretched hypothesis? [Editorial]. Lancet. 2001;357:405.

75. Fall C, Davies A, Motiwala A. Proceedings of the first world congress on the fetal origins of adult disease. Scientific programme and abstracts. Mumbia, India; 2001. 
76. Leon DA, Lithell HO, Vagero D, Koupilova I, Mohsen R, Berglund L, Lithell UB, McKeigue PM. Reduced fetal growth rate and increased risk of death from ischaemic heart disease: cohort study of 15000 Swedish men and women born 1915-29. BMJ. 1998;317:241-245.

77. Koupilova I, Leon DA, Vagero D. Can confouding by sociodemographic and behavioural factors explain the association between size at birth and blood pressure at age 50 in Sweden? $J$ Epidemiol Community Health. 1997;51:14-18.

78. Rosendaal FR, Bouter LM. Roaming through methodology (conclusion). XXXIX. The ultimate truth [in Dutch]. Ned Tijdschr Geneeskd. 2002;146:304-308. 
Chapter 7 


\section{Abbreviations | Fatty acid nomenclature and list of units and abbreviations}

$18: 2 \mathrm{n}-6$
$18: 3 \mathrm{n}-3$
$18: 3 \mathrm{n}-6$
$20: 3 \mathrm{n}-6$
$20: 3 \mathrm{n}-9$
$20: 4 \mathrm{n}-6$
$20: 5 \mathrm{n}-3$
$22: 4 \mathrm{n}-6$
$22: 5 \mathrm{n}-3$
$22: 5 \mathrm{n}-6$
$22: 6 \mathrm{n}-3$

AA

AGA

ANOVA

Apo

$\beta$

BMI

${ }^{\circ} \mathrm{C}$

CETP

$\mathrm{CI}$

DHA

DNA

EDTA

EFA

ELISA

EPA

$\mathrm{F}$

FAME

FFM

FM

$\mathrm{g}$

GA

GC linoleic acid

$\alpha$-linolenic acid

$\gamma$-linolenic acid

dihomo- $\gamma$-linolenic acid

eicosatrienoic acid or Mead acid

arachidonic acid

eicosapentaenoic acid or timnodonic acid

docosatetraenoic acid or adrenic acid

docosapentaenoic acid or clupanodonic acid

docosapentaenoic acid or Osbond acid

docosahexaenoic acid or cervonic acid

arachidonic acid

appropriate for gestational age

analysis of variance

apolipoprotein

regression coefficient

body mass index

degree Celsius

cholesteryl ester transfer protein

confidence interval

docosahexaenoic acid

deoxyribonucleic acid

ethylenediaminetetraacetic acid

essential fatty acid

enzyme-linked immunosorbent assay

eicosapentaenoic acid

female

fatty acid methyl ester

fat-free mass

fat mass

gram

gestational age

gas chromotagraphy 
h

HDL

HOMA

IQR

L

LCP

LC-PUFA

LDL

LGA

Lp(a)

M

$\mathrm{m}$

$\min$

mol

MUFA

$n$

ND

NIDDM

NS

$P$

PCR

PL

PUFA

$\mathrm{r}$

RIA

SD

SE

SFA

SGA

TG

VLDL

wk

wt

y hour

high-density lipoprotein

homeostasis model assessment

inter quartile range

liter

long-chain polyene

long-chain polyunsaturated fatty acid

low-density lipoprotein

large for gestational age

lipoprotein(a)

male

meter

minute

mole

monounsaturated fatty acid

number of subjects

not detected

non-insulin-dependent diabetes mellitus

not statistically significant

probability value

polymerase chain reaction

phospholipid

polyunsaturated fatty acid

correlation coefficient

radio-immunoassay

standard deviation

standard error

saturated fatty acid

small for gestational age

triacylglycerol

very low-density lipoprotein

week

weight

year 
Summary | Polyunsaturated fatty acids and the fetal origins hypothesis 
Cardiovascular diseases are the most important cause of death in the Dutch population. Although the mortality rates for cardiovascular diseases are declining, they remain a substantial public health problem. Previous research has shown that multiple factors play a role in the origins of cardiovascular diseases. For instance, it has become clear that hypertension, diabetes, hypercholesterolemia, and obesity increase the risk for cardiovascular diseases. Besides genetic factors, environmental factors like physical activity, smoking en nutrition play a role in the etiology. Usually, the symptoms of cardiovascular diseases occur for the first time during adulthood. In (young) children, however, early stages of the atherosclerotic process can be found during autopsy studies. Additionally, it is known that many children tend to maintain their ranking order with respect to several cardiovascular risk indicators from childhood into adulthood. Such observations support the idea that a combination of factors, acting since childhood, contributes to the origins of cardiovascular diseases.

In the past twenty years or so, research findings suggest that factors active during the short period of life before birth may increase the risk for adult cardiovascular diseases. Epidemiological studies in different populations have shown that a low birth weight is associated with an increased coronary heart disease mortality. Subjects with a low birth weight are also more likely to suffer from diabetes, hypertension, or dyslipidemia. Associations like these have led to the formulation of the fetal origins hypothesis. According to this hypothesis, the risk for chronic diseases in adult life is in part determined by the availability of nutrients during the fetal period. It is believed that this is a consequence of permanent physiological and structural adaptations of the body. The mechanisms involved are hardly understood, but results from animal experiments and observations in subjects exposed to the Dutch famine during the winter of 1944 1945 have supported the suggestion that fetal nutrient availability is important. Only a very small number of human studies have been conducted to identify the specific nutritional factors involved. The primary aim of the study described in the present thesis was to explore the potential involvement of prenatal essential fatty acid availability.

Essential fatty acids belong to the long-chain polyunsaturated fatty acids. Strictly speaking, only linoleic acid and alpha-linolenic acid are essential fatty acids. Because the human body lacks the required enzymes needed for the synthesis of linoleic acid and alpha-linolenic acid, these fatty acids have to be obtained through the diet. This means that a fetus has to receive these nutrients from the mother by placental transfer. Once obtained, linoleic acid and alpha-linolenic acid can be converted into other longchain polyunsaturated fatty acids, like gamma-linolenic acid, dihomo-gamma-linolenic acid, arachidonic acid, eicosapentaenoic acid, or docosahexaenoic acid. Because the fetal capacity for these conversions seems rather low, these products are also considered as essential fatty acids. Essential fatty acids have many biological functions. They are not just a source of energy, but are also active mediators of physiological processes and regulators of gene expression. They may conduct such functions directly 
or indirectly. Observations from previous studies suggest that fetal essential fatty acid availability is limited. Furthermore, essential fatty acids seem to have an influence on the intrauterine growth of the fetus. This renders them as attractive candidate nutrients in relation to the fetal origins hypothesis.

To investigate the relation between fetal essential fatty acid availability and later cardiovascular disease risk, a cohort of children was studied at seven years of age. The mothers of these children had previously participated in a study on maternal and fetal essential fatty acid status and pregnancy outcome. From the children of this cohort the essential fatty acid composition of umbilical cord plasma phospholipids was known. The fatty acid composition of cord plasma phospholipids can be used as an indicator of fetal essential fatty acid availability. In total, 297 of the eligible 750 children participated in the follow-up study on cardiovascular risk indicators. More information on the rationale for the study, design of the study, and participation is given in general introduction of this thesis (Chapter 1).

In Chapter 2, the means and distributions are reported for the fatty acid concentrations measured in the phospholipid fraction of cord plasma and maternal plasma at the time of birth. As described previously, the absolute fatty acid concentrations (expressed in $\mathrm{mg} / \mathrm{L}$ ) were significantly higher in maternal plasma than in umbilical cord plasma. In addition we observed that boys had lower absolute fatty acid concentrations than girls had. Both observations are probably the result of a difference in the size of the phospholipid pool. The relative fatty acid concentrations (expressed in $\%$ of total fatty acids) did not differ between boys and girls. The well known differences in relative fatty acid concentrations were observed between mothers and children, namely a higher content of linoleic acid and alpha-linolenic acid and lower concentrations of the derived products dihomo-gamma-linolenic acid, arachidonic acid, eicosapentaenoic acid, and docosahexaenoic acid in mothers. The relative concentration of docosahexaenoic acid in umbilical cord plasma was related to the duration of pregnancy and maternal dietary linoleic acid intake. Mothers with a higher intake of linoleic acid during pregnancy more frequently had children with a lower docosahexaenoic acid concentration appropriate for gestational age than mothers with a lower linoleic acid intake.

In order to investigate the relation between the fetal essential fatty acid availability and later cardiovascular disease risk, we first studied the association between birth weight and relative essential fatty acid concentrations in umbilical cord plasma (Chapter 3). Results from previous studies in premature infants and low-birthweight neonates (weight $<2500$ gram) indicated that the overall biochemical essential fatty acid status of a child is positively related to the weight attained at birth. In our study however, heavier infants had a lower biochemical essential fatty acid status that lighter infants. Dihomo-gamma-linolenic acid was the only essential fatty acid positively related to birth weight (dihomo-gamma-linolenic acid is also positively 
related to size at birth in premature infants and low-birth-weight neonates). In addition, we observed a decline in maternal concentrations of n-6 and n-3 long-chain polyunsaturated fatty acids (e.g. arachidonic acid and docosahexaenic acid) during pregnancy. This decrease was most pronounced in mothers of heavier infants. Observations like these seem to suggest that more fatty acids are transported from the mother to the fetus via the placenta when the fetus is relatively large. Because the biochemical essential fatty acid status is lower in heavier infants compared to lighter infants, the capacity for the maternal-to-fetal delivery of essential fatty acids seems limited. Based on the results of this study, it seems less likely that the general biochemical essential fatty acid status at birth is negatively related to the cardiovascular risk profile at seven years of age. For an adverse risk profile is related to a low weight at birth. However, the involvement of the availability of individual fatty acids (like dihomo-gamma-linolenic acid) or a relation with essential fatty acid status independent of birth weight could still be important.

Chapter 4 and Chapter 5 describe the relations between essential fatty acid concentrations in umbilical cord plasma and several cardiovascular risk indicators determined at age seven. A relation was found with the concentrations of two essential fatty acids. A higher concentration of gamma-linolenic acid and dihomo-gammalinolenic acid in umbilical cord plasma was associated with lower fasting concentrations of triacylglycerol and insulin at seven years of age. A higher concentration of gamma-linolenic acid was also related to lower levels of proinsulin and leptin, and to a lower percentage of body fat. The concentration of gamma-linolenic acid and dihomo-gamma-linolenic acid in umbilical cord plasma were both negatively related to the homeostasis model assessment (HOMA) index for insulin resistance. Together these findings suggest that the fetal availability of gamma-linolenic acid and/or dihomo-gamma-linolenic acid could be of importance in the development of Syndrome X (also called the metabolic syndrome or the insulin resistance syndrome). An implication of this might be that a higher maternal intake of these fatty acids during pregnancy could lower the risk of insulin resistance in her offspring.

We did not find associations between umbilical cord plasma concentrations of gamma-linolenic acid and dihomo-gamma-linolenic acid and plasma concentrations of total cholesterol, LDL cholesterol, HDL cholesterol, apolipoprotein A, apolipoprotein B, and lipoprotein(a) at seven years of age. Umbilical cord plasma concentrations of linoleic acid, arachidonic acid, eicosapentaenoic acid, and docosahexaenoic acid were not related to the measured risk indicators for cardiovascular diseases (Chapters $4 \& 5$ ). Markers for the general essential fatty acid status (e.g. Mead acid or the essential fatty acid status index) did not relate to the cardiovascular risk indicators either. It seems therefore that the availability of individual fatty acids (in particular gamma-linolenic acid and dihomo-gamma-linolenic acid) is more important than the essential fatty acid status at birth in general. 
A relation between the fetal availability of essential fatty acids and the later risk for cardiovascular diseases could be influenced by genetic differences between subjects. For this reason, two common gene variations were studied, i.e. the Taq IB polymorphism in the cholesteryl ester transfer protein (CETP) gene and the apolipoprotein E polymorphism. Both polymorphisms, however, proved not to be informative in relation to this hypothesis. We did observe the expected associations with plasma concentrations of lipids and lipoproteins. In addition, an interaction between these genetic variants was seen (see Chapter 6).

The limitations of the study are discussed in detail in the general discussion section of this thesis (Chapter 7). Discussed are the use of plasma phospholipid fatty acid concentrations as a proxy for essential fatty acid availability, de potential consequences of the low participation rate, the possibility of confounding, and alternative hypothesis to explain some of our findings. In light of these limitations a serious recommendation can not be given regarding the maternal intake of gammalinolenic acid and dihomo-gamma-linolenic acid during pregnancy. Preferably, the effect of an increased maternal intake of these fatty acids during pregnancy on the cardiovascular risk profile of the next generation is tested during future, more controlled, randomized trials. In this way, it could also become clear whether the associations found reflect a causative relation indeed. 
Summary 
Samenvatting | Meervoudig onverzadigde vetzuren en de fetal origins hypothese 


\section{Samenvatting}

Hart- en vaatziekten vormen de belangrijkste oorzaak van overlijden in de Nederlandse bevolking. Hoewel de sterfte aan coronaire hartziekten daalt, blijven deze aandoeningen een aanzienlijk volksgezondheidsprobleem. Uit eerder onderzoek is gebleken dat vele factoren een rol spelen bij het ontstaan van hart- en vaataandoeningen. Zo is duidelijk geworden dat o.a. hypertensie, diabetes, hypercholesterolemie en obesitas het risico op hart- en vaatziekten verhogen. Naast erfelijke factoren spelen ook omgevingsfactoren zoals lichamelijke activiteit, roken en voeding een rol bij de etiologie. De klachten en symptomen van hart- en vaatziekten komen meestal pas op volwassen leeftijd tot uiting. Echter op de (vroege) kinderleeftijd kunnen bij obductie reeds de eerste tekenen van atherosclerose worden waargenomen. Daarnaast is bekend dat veel kinderen hun rangorde ten aanzien van verschillende risico-indicatoren voor hart- en vaatziekten handhaven tot op volwassen leeftijd. Dergelijke bevindingen ondersteunen het idee dat een combinatie van factoren al vanaf de kinderleeftijd bijdraagt aan het ontstaan van hart- en vaatziekten.

Gedurende de laatste twee decennia zijn er aanwijzingen gevonden dat ook

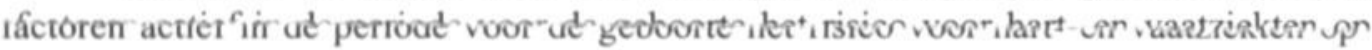
volwassen leeftijd kunnen verhogen. Epidemiologische studies in verschillende populaties hebben laten zien dat mensen met een laag geboortegewicht op latere leeftijd een grotere kans hebben om te overlijden aan de gevolgen van coronaire hartziekten. Ook hebben zij vaker last van diabetes, hypertensie en hypercholesterolemie. De gevonden associaties hebben geleid tot de formulering van de "fetal origins" hypothese. Volgens deze hypothese bepaalt de beschikbaarheid van voeding tijdens de foetale periode mede het risico op chronische aandoeningen in het latere leven. Dit zou het gevolg zijn van permanente structurele en fysiologische aanpassingen in het lichaam. Welke mechanismen hierbij een rol spelen is nog nauwelijks bekend, maar dat de beschikbaarheid van voedingsstoffen tijdens de foetale periode inderdaad belangrijk kan zijn, werd ondersteund met behuip van dierexperimentele studies en observaties bij mensen die voor hun geboorte waren blootgesteld aan de hongerwinter in $1944-1945$. Welke specifieke voedingsfactoren hierbij een rol spelen is tot nu toe nog nauwelijks bestudeerd. Het onderzoek beschreven in dit proefschrift had als hoofddoel het verkennen van een mogelijke betrokkenheid van de prenatale beschikbaarheid van essentiële vetzuren.

Essentiële vetzuren behoren tot de lange-keten meervoudig onverzadigde vetzuren. In strikte zin zijn alleen linolzuur en alfa-linoleenzuur essentiële vetzuren. Door het ontbreken van de benodigde enzymen kunnen linolzuur en alfa-linoleenzuur niet door het lichaam worden gevormd, daarom moeten zij via de voeding worden opgenomen. Dit betekent dat een foetus deze voedingsstoffen van de moeder moet krijgen via transport door de placenta. Eenmaal in het lichaam kunnen linolzuur en alfalinoleenzuur worden omgezet in andere lange-keten meervoudig onverzadigde vetzuren, zoals gamma-linoleenzuur, dihomo-gamma-linoleenzuur, arachidonzuur, eicosapentaeenzuur en docosahexaeenzuur. Omdat de foetale capaciteit voor deze 
omzettingen beperkt lijkt te zijn, worden deze vetzuren vaak ook tot de essentiēle vetzuren gerekend. Essentiële vetzuren vervullen een groot aantal biologische functies. $\mathrm{Zij}$ vormen niet alleen een bron van energie, maar reguleren direct of indirect ook fysiologische processen en beïnvloeden de expressie van genen. Eerder onderzoek doet vermoeden dat de foetale beschikbaarheid van essentielle vetzuren beperkt is. Ook zouden essentiële vetzuren een invloed hebben op de intra-uteriene groei van de foetus. Dit maakt dat zij interessante kandidaat-voedingsstoffen zijn in relatie tot de fetal origins hypothese.

Om het verband tussen de foetale beschikbaarheid van essentiële vetzuren en het latere risico op hart- en vaatziekten te bestuderen, werd een cohort kinderen op 7-jarige leeftijd onderzocht. De moeders van deze kinderen hadden eerder deelgenomen aan een onderzoek naar de maternale en neonatale vetzuurstatus en de zwangerschapsuitkomst. Van de kinderen in dit cohort was bij de geboorte de essentiële vetzuursamenstelling van fosfolipiden in navelstrengplasma bekend. Deze vetzuursamenstelling van fosfolipiden kan worden gebruikt als een indicator voor de foetale beschikbaarheid van essentiële vetzuren. Van de oorspronkelijk 750 kinderen in het cohort namen er uiteindelijk 297 deel aan het vervolgonderzoek naar risico-indicatoren voor hart- en vaatziekten. Meer informatie over de achtergronden van deze studie, de studieopzet en de participatie is gegeven in de algemene inleiding van dit proefschrift (Hoofdstuk l).

In Hoofdstuk 2 worden gemiddelden en spreiding van de gemeten vetzuurconcentraties in fosfolipiden van navelstrengplasma en moederlijk plasma ten tijde van de bevalling gerapporteerd. Zoals eerder al beschreven is, waren de absolute concentraties (uitgedrukt in $\mathrm{mg} / \mathrm{L}$ ) van de vetzuren hoger bij de moeders dan bij hun kinderen. Verder zagen we dat jongens iets lagere absolute vetzuurconcentraties hadden dan meisjes. Beide observaties worden waarschijnlijk verklaard door een verschil in de grootte van de fosfolipidenpool waarin gemeten is. De relatieve vetzuurwaarden (uitgedrukt in \% van de totale hoeveelheid vetzuren) verschilden niet tussen jongens en meisjes. Tussen moeders en kinderen werden de bekende verschillen in relatieve vetzuurconcentraties waargenomen, namelijk een hogere concentratie van linolzuur en alfa-linoleenzuur en lagere concentraties van de afgeleide vetzuren dihomo-gammalinoleenzuur, arachidonzuur, eicosapentaeenzuur, en docosahexaeenzuur bij moeders. De relatieve concentratie van docosahexaeenzuur in navelstreng plasma was afhankelijk van de duur van de zwangerschap alsmede de moederlijke inname van linolzuur. Moeders met een hogere inname van linolzuur in de zwangerschap hadden vaker een kind met een lagere docosahexaeenzuurconcentratie in navelstrengplasma voor de duur van de zwangerschap dan moeders met een lagere linolzuurinname.

Om het verband tussen de foetale beschikbaarheid van essentiële vetzuren en het latere risico op hart- en vaatziekten te bestuderen, werd in eerste instantie gekeken naar de relatie tussen de relatieve essentiële vetzuurconcentraties in navelstrengplasma en het geboortegewicht (Hoofdstuk 3). Resultaten van eerdere studies bij prematuur 


\section{Samenvatting}

geboren kinderen en kinderen met een laag geboortegewicht $(<2500$ gram $)$ deden vermoeden dat in het algemeen de biochemische essentiële vetzuurstatus positief gerelateerd is met het geboortegewicht van een kind. Wij vonden in ons cohort van à term geboren kinderen echter dat zwaardere kinderen bij geboorte een lagere biochemische essentiële vetzuurstatus hadden dan lichtere kinderen. Het enige essentiële vetzuur dat een positieve associatie had met geboortegewicht was dihomogamma-linoleenzuur (dihomo-gamma-linoleenzuur is ook bij prematuur geboren kinderen positief gerelateerd aan het geboortegewicht). Verder zagen we dat de concentraties van n-6 en n-3 lange-keten meervoudig onverzadigde vetzuren (zoals arachidonzuur en docosahexaeenzuur) in moederlijk plasma daalden gedurende de zwangerschap. Deze daling was sterker bij moeders van zwaardere baby's. Deze observaties suggereren dat er meer essentiële vetzuren van moeder naar kind worden getransporteerd via de placenta als het kind relatief zwaarder is. Omdat de biochemische vetzuurstatus van zwaardere kinderen lager is dan die van lichtere kinderen, lijkt de capaciteit voor deze aanvoer beperkt te zijn. De resultaten van deze studie maken het minder waarschijnlijk dat de biochemische essentiële vetzuurstatus in het algemeen negatief gerelateerd zou zijn aan het risicoprofiel voor hart- en vaatziekten op 7-jarige leeftijd. Een ongunstig risicoprofiel is immers gerelateerd aan een laag geboortegewicht. Echter de betrokkenheid van de beschikbaarheid van individuele vetzuren (zoals dihomo-gamma-linoleenzuur) of een relatie met essentiële vetzuren onafhankelijk van geboortegewicht behoren nog steeds tot de mogelijkheden.

Hoofdstuk 4 en Hoofdstuk 5 beschrijven de relaties tussen essentiële vetzuurconcentraties in navelstrengplasma en verschillende risico-indicatoren voor hart- en vaatziekten gemeten op 7-jarige leeftijd. Een associatie met de concentraties van twee essentiële vetzuren werd gevonden. Een hoger gehalte aan gammalinoleenzuur en dihomo-gamma-linoleenzuur in navelstrengplasma relateerde aan een lager nuchter gemeten plasma concentratie van triacylglycerol en insuline op 7-jarige leeftijd. Een hogere concentratie van gamma-linoleenzuur was ook gerelateerd aan een lagere concentratie pro-insuline en leptine, en een lager lichaamsvetpercentage. De concentraties van gamma-linoleenzuur en dihomo-gamma-linoleenzuur in navelstrengplasma waren beiden negatief gerelateerd aan de "homeostasis model assesment" (HOMA) index voor insulineresistentie. Samen suggereren deze bevindingen dat de foetale beschikbaarheid van gamma-linoleenzuur en/of dihomogamma-linoleenzuur van belang zou zijn voor de latere ontwikkeling van "Syndrome X" (ook wel "the metabolic syndrome" of "the insulin resistance syndrome" genoemd). Een implicatie hiervan kan zijn dat een hogere inname van deze vetzuren bij zwangere vrouwen het latere risico op insulineresistentie bij hun kinderen zou kunnen verlagen.

Er werden geen relaties gevonden tussen de navelstrengconcentraties van gamma-linoleenzuur en dihomo-gamma-linoleenzuur en de plasma concentraties van totaal cholesterol, LDL-cholesterol, HDL-cholesterol, apolipoproteine A, apolipoproteỉne B en lipoproteïne(a) op 7-jarige leeftijd. De navelstreng- 
plasmaconcentraties van linolzuur, arachidonzuur, eicosapentaeenzuur en docosahexaeenzuur waren niet gerelateerd aan de gemeten risico-indicatoren voor hart- en vaatziekten op 7-jarige leeftijd (Hoofdstukken $4 \& 5$ ). Ook markers voor een algemeen lagere biochemische essentiële vetzuurstatus bij geboorte (zoals Meadzuur en de essentiële vetzuurstatusindex) waren niet gerelateerd aan risico-indicatoren gemeten op 7-jarige leeftijd. Het lijkt daarom dat de beschikbaarheid van individuele vetzuren (in het bijzonder gamma-linoleenzuur en dihomo-gamma-linoleenzuur) belangrijker is dan de algemene biochemische essentiële vetzuurstatus bij geboorte.

Een verband tussen de foetale beschikbaarheid van essentiële vetzuren en het latere risico op hart- en vaatziekten zou afhankelijk kunnen zijn van genetische verschillen tussen personen. Om deze reden werden ook twee veelvoorkomende genetische variaties bestudeerd, namelijk het Taq IB polymorfisme in het gen dat codeert voor het cholesterylester transfer proteïne (CETP) en het polymorfisme in het apolipoproteïne E gen. Beiden bleken echter niet informatief voor de bovenstaande vraagstelling. Wel vonden we de te verwachten associaties met plasmaconcentraties van lipiden en lipoproteïnes op 7-jarige leeftijd en zagen we een interactie tussen deze genetische varianten (zie Hoofdstuk 6).

In Hoofdstuk 7 worden de verschillende beperkingen van het onderzoek in detail besproken. Er wordt ondermeer ingegaan op het gebruik van vetzuurconcentraties in plasma fosfolipiden als maat voor de beschikbaarheid van essentiële vetzuren, de potentiële gevolgen van de beperkte deelname aan het onderzoek, een mogelijke vertekening van de resultaten en alternatieve verklaringen voor onze bevindingen. Met het oog op deze beperkingen, is een serieuze aanbeveling over de maternale inname van gamma-linoleenzuur en dihomo-gamma-linoleenzuur gedurende de zwangerschap nog niet te geven. Bij voorkeur zouden de effecten van een verhoogde inname van deze vetzuren tijdens de zwangerschap op het risicoprofiel voor hart- en vaatziekten in de volgende generatie in toekomstige, meer gecontroleerde, studies moeten worden onderzocht. Op deze manier zou ook meer duidelijkheid verschaft kunnen worden ten aanzien van de vraag of de gevonden associaties inderdaad een causaal verband weergeven. 
Samenvatting 


\section{Dankwoord |}

Veel personen hebben een belangrijke bijdrage geleverd aan de totstandkoming van dit proefschrift. Een aantal van deze mensen wil ik met name noemen.

Allereerst wil ik alle kinderen die mee hebben gedaan aan het onderzoek, en natuurlijk ook hun ouders, bedanken. Zonder jullie enthousiaste inzet waren de studies beschreven in dit proefschrift onmogelijk geweest.

Gerard Hornstra, mijn promotor. Jou wil ik bedanken voor het vertrouwen, de altijd laagdrempelige begeleiding, de interessante discussies, de prettige samenwerking, de kritische kanttekeningen, de snelle beoordeling van manuscripten, het aanbrengen van smeerolie op de soms logge onderzoeksmachine, en natuurlijk ook voor de vele gezellige etentjes. Ik zal de "kip" die wij in Tsukuba voorgeschoteld kregen, en die wij alleen met veel Sake konden wegspoelen, niet gauw vergeten.

Ook wil ik Ronald Mensink, mijn tweede promotor, bedanken voor zijn essentiële bijdrage. Jouw hulp bij het organiseren en realiseren van de laboratoriumanalyses, de plezierige samenwerking, en jouw "meedenken" over de verschillende onderdelen van mijn project waren onontbeerlijk.

Esther Bakker, mijn kamergenote en directe collega op het project. Ik heb altijd met veel plezier met jouw samengewerkt. Jij hebt veel werk verzet waarvan ook ik geprofiteerd heb. Als twee "Hollanders" hebben wij, soms met Babylonische spraakverwarringen, onze Limburgse proefpersoontjes aangemoedigd om de voor hun vaak enge onderdelen van het onderzoek te volbrengen. Met elkaar hebben wij deze klus toch maar mooi geklaard! Ook wil ik Monique Al bedanken. Zij heeft met haar onderzoek de basis voor het hele project gelegd.

De vele analisten, die ieder een grote dan wel een kleine bijdrage hebben geleverd. Jo ( $\dagger$ ), Dennis, Martijn, Maurice, Sjoerd, René, Frank, Hasibe, Joan en Jos hartelijk bedankt! Ook Jeroen van den Berg (Numico, Wageningen), die vele vetzuuranalyses heeft verricht, wil ik niet ongenoemd laten.

Veel proefpersoontjes en hun ouders leken van de aardbodem verdwenen te zijn toen wij aan het project begonnen. Maar dankzij de Pitbullmentaliteit op het secretariaat hebben we ze kunnen traceren. Hiervoor wil ik Marco, Mehnaz en met name ook Peggy bedanken. Inge, jouw wil ik bedanken voor de navelstrengachtige band met de vakgroep die ik na mijn vertrek naar Groningen via jou kon onderhouden. 
Ik wil prof. dr E. Mariman, prof. dr T.W.A. de Bruin, dr J.H.P. van der Meulen, prof. dr F.A.J. Muskiet en prof. dr ir W.H.M. Saris bedanken voor de tijd die zij genomen hebben om het manuscript te beoordelen.

De medewerkers van de Polikliniek Kindergeneeskunde en de afdeling Bewegingswetenschappen bedank ik voor hun gastvrijheid. Dr W.J.M. Gerver wil ik bedanken voor de spoedcursus anthropometrie, en voor zijn bijdrage aan het artikel over de inspanningstest. Ook Frans Verstappen wil ik danken voor zijn grote aandeel in dit onderdeel van het project. Dr H.J. Hendriks dank ik voor zijn inbreng in het "atopie gedeelte" van het project, zijn suggesties voor het onderzoeksprotocol, en voor het beschikbaar stellen van de peakflow-metertjes. Helaas liggen de waardevolle resultaten van dit deel van het project nog te wachten op nadere analyse.

Arnold Kester bedank ik voor zijn statistische adviezen. Alex Ghys, jij hebt je als klinisch psycholoog in vetzuren verdiept en wij hebben vaak samen gefilosofeerd over de vragen die wij onszelf stelden. Bedankt voor jouw reflectieve inbreng en jouw bemoedigende opmerkingen. De studenten Jildou, Hester, Miriam en Diane wil ik ook bedanken voor hun inzet. Ik heb vaak geprofiteerd van jullie werk.

Loek en Paul, bedankt voor de onmisbare ICT-ondersteunende werkzaamheden en natuurlijk de altijd fascinerende maar vaak onbegrijpelijke e-mailtjes. Verder wil ik ook de nog niet eerder genoemde leden van het "perinat-praatgroepje" danken voor de altijd weer interessante discussies. Suzie, Renate, en Rian bedankt! Verder dank ik alle andere collega's van de vakgroep Humane Biologie voor hun hulp, advies, en belangstelling.

Mijn nieuwe collega's en opleiders in Groningen wil ik nog bedanken voor het vertrouwen dat ze mij gaven en de ruimte die zij mij boden om het proefschrift te kunnen voltooien. Ik hoop dat ik nog lang met jullie mag samenwerken. Er gaat niets boven Groningen!

Ook wil ik alle familieleden en vrienden die belangstelling toonden voor mijn wetenschappelijke werkzaamheden bedanken. Astrid en Armand, jullie wil ik speciaal bedanken voor de altijd hartelijke wijze waarop jullie mij en Susanne hebben ontvangen. Dankzij jullie hebben wij het echte Maastricht leren kennen. Mijn woordenschat is mede door jullie op Maastrichtse wijze opgekleurd. Armand, ik ben blij dat jij tijdens de verdediging als paranimf achter mij staat.

Lang voor ik de universiteitsdeuren passeerde heb ik al mijn eerste belangrijke lessen geleerd. Ik wil daarom ook mijn ouders, broer en zusje bedanken voor het leggen van de eerste stenen in de fundering van mijn opleiding. Lies, Frans, Roy en Renate, hartelijk bedankt! Jullie hebben mij de mogelijkheid gegeven. Dit geldt natuurlijk ook voor jouw, Dieter. Renate, het is fantastisch dat je mijn paranimf wilt zijn. Susanne, jij gaf mij alle steun, liefde, en hulp wanneer dat nodig was. Al ruim een kwart van jouw leven heb jij met mij willen delen. Ik hoop dat wij uiteindelijk slechts een fractie van jouw leven niet gedeeld hebben. 


\section{Curriculum Vitae |}

Patrick Rump was born in Sliedrecht on September $25^{\text {th }}$ in 1967. After completing high school (HAVO) at St. Canisius in Almelo in 1985, and college (VWO) at Pius X College in Almelo in 1987, he studied Medicine and Biology at the University of Nijmegen. As a student he performed studies on in situ fibre-optic oxygen measurement, on ion transport mechanisms in cell volume regulation, and on cellular magnesium homeostasis, at the Departments of Physiology and Cell Physiology of the Faculty of Medicine, and at the Department of Animal Physiology of the Faculty of Science, respectively. In addition, he worked on a research project involving the PRimed In Situ (PRINS) labelling technique for metaphase chromosomes at the MaxPlanck Institute for Molecular Genetics in Berlin. He has a Master of Science degree in Biology from the University of Nijmegen (recieved in 1995) and finished his medical training at St. Radboud University Hospital in Nijmegen in 1996. During the following years he worked as a PhD-fellow for the Department of Human Biology at the Nutrition and Toxicology Research Institute Maastricht (NUTRIM) of the University of Maastricht. The results of the research performed during this period are for the greater part described in the present thesis. Patrick Rump is currently working as a resident in Clinical Genetics at the Department of Clinical Genetics of the Academic Hospital in Groningen. 


\title{
Publications | List of publications and submitted manuscripts
}

\author{
Full papers
}

P. Rump, B.C.J. Hamel, A.J.L.G. Pinckers, P.A. van Dop. Two sibs with chorioretinal dystrophy, hypogonadotrophic hypogonadism, and cerebellar ataxia: Boucher-Neuhăuser syndrome. J Med Genet. 1997;34:767-771

P. Rump, M.Y.C. Gruijters, C.J.A.M. van der Burgt. A female patient with neurological, facial, digital and renal abnormalities: another case of the neurofacialdigitorenal (NFDR) syndrome? Clin Dysmorphol. 1997;6:337-340

P. Rump, R.P. Mensink, A.D.M. Kester, G. Hornstra. Essential fatty acid composition of plasma phospholipids and birth weight: a study in term neonates. Am J Clin Nutr. 2001;73:797-806

P. Rump, S.J. Otto, G. Hornstra. Leptin and phospholipid-esterified docosahexaenoic acid concentrations in plasma of women: observations during pregnancy and lactation. Eur J Clin Nutr. 2001;55:244-251

P. Rump, F. Verstappen, W.J.M. Gerver, G. Hornstra. Body composition and cardiorespiratory fitness indicators in prepubescent boys and girls. Int J Sports Med. 2002;23:50-54

P. Rump, G. Hornstra. The $n-3$ and $n-6$ polyunsaturated fatty acid composition of plasma phospholipids in pregnant women and their infants. Relationship with maternal linoleic acid intake. Clin Chem Lab Med. 2002;40:32-39

P. Rump, C. Popp-Sneiders, R.J. Heine, G. Hornstra. Components of the insulin resistance syndrome in seven-year-old children: relations with birth weight and the polyunsaturated fatty acid content of umbilical cord plasma phospholipids. Diabetologia. 2002;45:349-355

P. Rump, R.P. Mensink, G. Hornstra. Interaction between a common variant of the cholesteryl ester transfer protein gene and the apolipoprotein E polymorphism: effects on plasma lipids and lipoproteins in a cohort of 7-year-old children. Nutr Metab Cardiovasc Dis. (in press)

P. Rump, R.P. Mensink, G. Hornstra. Fasting triacylglycerol concentrations in 7-year-old children are related to the polyunsaturated fatty acid composition of umbilical cord plasma phospholipids at birth. Submitted for publication

Ghys, E. Bakker, P. Rump, G. Hornstra, M. van den Hout. Socio-economic status and longchain polyunsaturated fatty acids. Submitted for publication 


\section{Letters}

P. Rump, G. Hornstra. Relation between arachidonic acid and docosahexaenoic acid in maternal and neonatal blood. Eur J Clin Nutr. 2001;55:916-917

\section{Abstracts}

G. Fons, R. Engbersen, P. Rump, H. van der Grinten. Can we use inline venous saturation measurements for reliable calculation of oxygen uptake. Proceedings of the XVIII congress of the European Society for Artificial Organs. Vienna, 1991

N.J.H. Raat, E. Delpire, P. Rump, C.H. van Os, R.J.M. Bindels. Difference in expression of $\mathrm{Na}-\mathrm{K}-2 \mathrm{Cl}$ co-transport between freshly isolated and primary cultured rabbit proximal tubule cells. J Gen Physiol. 1994;104: 51 A

P. Rump, R.P. Mensink, A.D.M. Kester, G. Hornstra. Essential fatty acid composition of plasma phospholipids and weight at birth: a study in term neonates. Proceedings of the $4^{\text {th }}$ Congress of the International Society for the Study of Fatty Acids and Lipids (ISSFAL).

Tsukuba, 2000

G. Hornstra, A.C. van Houwelingen, S.J. Otto, P. Rump, and E.C. Bakker. Maternal, fetal, and neonatal essential fatty acids: Mutual interactions and developmental aspects. Proceedings of the $4^{\text {th }}$ Congress of the International Society for the Study of Fatty Acids and Lipids (ISSFAL). Tsukuba, 2000

P. Rump, G. Hornstra, C. Popp-Snijders, R.J. Heine. Relationship between essential fatty acid status at birth and components of the insulin resistance syndrome in 7-year-old children. Proceedings of the $5^{\text {th }}$ Congress of the International Society for the Study of Fatty Acids and Lipids (ISSFAL). Montreal, 2002 
Appendices | Maternal polyunsaturated fatty acid concentrations during the first, second, and third trimester of pregnancy $\mid A, B$ \& C

Polyunsaturated fatty acid concentrations in seven-year-old boys and girls $\mid D \& E$

Anthropometric characteristics and cardiovascular risk indicator levels in seven-year-old boys and girls $\mid \mathrm{F}$

Graphics on the distribution of umbilical cord plasma fatty acid concentrations in relation to gestational age at birth $\mid \mathbf{G}$ 
Appendix A: The polyunsaturated fatty acid composition of maternal plasma phospholipids during the first trimester of pregnancy ( $n=837){ }^{1}$

\begin{tabular}{|c|c|c|c|c|c|c|c|c|c|c|c|c|c|c|}
\hline & \multicolumn{7}{|c|}{$m g / L$} & \multicolumn{7}{|c|}{$\% w t w t$} \\
\hline & \multirow[t]{2}{*}{ Mean } & \multirow[t]{2}{*}{ SD } & \multicolumn{5}{|c|}{ Percentile } & \multirow[t]{2}{*}{ Mean } & \multirow[t]{2}{*}{ SD } & \multicolumn{5}{|c|}{ Percentile } \\
\hline & & & 10 & 25 & 50 & 75 & 90 & & & 10 & 25 & 50 & 75 & 90 \\
\hline \multicolumn{15}{|l|}{ Fatty acid } \\
\hline $18: 2 n-6$ & 283.22 & 58.17 & 214.20 & 241.92 & 277.90 & 317.01 & 357.58 & 21.39 & 2.65 & 18.00 & 19.67 & 21.27 & 23.16 & 24.90 \\
\hline $18: 3 n-6$ & 0.51 & 0.42 & 0.00 & 0.27 & 0.43 & 0.67 & 1.02 & 0.04 & 0.03 & 0.00 & 0.02 & 0.03 & 0.05 & 0.07 \\
\hline $20: 2 n-6$ & 6.17 & 1.79 & 3.92 & 4.88 & 6.00 & 7.33 & 8.52 & 0.46 & 0.09 & 0.35 & 0.40 & 0.45 & 0.51 & 0.58 \\
\hline $20: 3 n-6$ & 41.91 & 12.78 & 26.08 & 32.74 & 40.96 & 49.91 & 57.89 & 3.13 & 0.66 & 2.33 & 2.67 & 3.07 & 3.54 & 4.02 \\
\hline $20: 4 n-6$ & 127.42 & 29.65 & 92.13 & 106.50 & 123.94 & 145.63 & 166.48 & 9.60 & 1.46 & 7.66 & 8.65 & 9.59 & 10.53 & 11.38 \\
\hline $22: 2 n-6$ & 0.18 & 0.19 & 0.00 & 0.00 & 0.18 & 0.27 & 0.34 & 0.01 & 0.01 & 0.00 & 0.00 & 0.01 & 0.02 & 0.03 \\
\hline $22: 4 n-6$ & 5.17 & 1.53 & 3.47 & 4.09 & 4.91 & 6.08 & 7.27 & 0.39 & 0.08 & 0.29 & 0.33 & 0.39 & 0.44 & 0.50 \\
\hline $22: 5 n-6$ & 4.55 & 2.02 & 2.23 & 3.00 & 4.21 & 5.71 & 7.27 & 0.34 & 0.12 & 0.19 & 0.25 & 0.33 & 0.41 & 0.50 \\
\hline $24: 2 n-6$ & 2.28 & 0.83 & 1.34 & 1.68 & 2.16 & 2.71 & 3.46 & 0.17 & 0.06 & 0.11 & 0.13 & 0.17 & 0.21 & 0.25 \\
\hline$\sum n-6$ & 471.65 & 86.37 & 364.08 & 411.44 & 464.30 & 525.52 & 581.11 & 35.56 & 1.99 & 32.94 & 34.19 & 35.65 & 36.87 & 38.04 \\
\hline$\sum n-6 L C P$ & 179.05 & 40.30 & 129.85 & 149.07 & 175.05 & 206.27 & 232.56 & 13.46 & 1.63 & 11.28 & 12.36 & 13.52 & 14.54 & 15.47 \\
\hline $18: 3 n-3$ & 2.74 & 1.65 & 1.11 & 1.70 & 2.55 & 3.45 & 4.56 & 0.21 & 0.12 & 0.09 & 0.14 & 0.20 & 0.26 & 0.33 \\
\hline $20: 3 n-3$ & 0.45 & 0.37 & 0.00 & 0.28 & 0.45 & 0.61 & 0.76 & 0.03 & 0.03 & 0.00 & 0.02 & 0.03 & 0.04 & 0.06 \\
\hline $20: 4 n-3$ & 1.88 & 1.02 & 0.87 & 1.27 & 1.68 & 2.34 & 3.10 & 0.14 & 0.07 & 0.08 & 0.10 & 0.13 & 0.17 & 0.22 \\
\hline $20: 5 n-3$ & 7.34 & 5.26 & 3.31 & 4.44 & 6.04 & 8.43 & 12.71 & 0.55 & 0.38 & 0.28 & 0.35 & 0.46 & 0.62 & 0.87 \\
\hline $22: 5 n-3$ & 9.86 & 2.72 & 6.80 & 7.89 & 9.46 & 11.41 & 13.50 & 0.75 & 0.17 & 0.53 & 0.62 & 0.74 & 0.85 & 0.96 \\
\hline $22: 6 n-3$ & 53.33 & 16.34 & 34.01 & 42.06 & 51.03 & 62.82 & 73.94 & 3.99 & 0.88 & 2.97 & 3.34 & 3.89 & 4.52 & 5.18 \\
\hline$\sum n-3$ & 75.60 & 22.28 & 50.91 & 59.54 & 72.47 & 86.75 & 103.19 & 5.67 & 1.19 & 4.41 & 4.88 & 5.46 & 6.25 & 7.08 \\
\hline$\sum n-3 L C P$ & 72.41 & 21.81 & 48.16 & 57.21 & 69.16 & 83.59 & 98.88 & 5.43 & 1.19 & 4.17 & 4.65 & 5.24 & 5.99 & 6.90 \\
\hline $20: 3 n-9$ & 2.57 & 1.33 & 1.19 & 1.62 & 2.35 & 3.29 & 4.34 & 0.19 & 0.10 & 0.10 & 0.13 & 0.18 & 0.24 & 0.32 \\
\hline$\sum n-7+n-9$ & 157.95 & 32.58 & 118.34 & 134.66 & 154.95 & 178.14 & 198.14 & 11.90 & 1.23 & 10.33 & 11.03 & 11.86 & 12.72 & 13.49 \\
\hline$\sum$ PUFA & 550.19 & 100.10 & 427.29 & 478.92 & 546.28 & 609.86 & 676.27 & 41.45 & 1.52 & 39.55 & 40.41 & 41.50 & 42.49 & 43.31 \\
\hline$\sum$ MUFA & 157.23 & 32.23 & 117.39 & 133.81 & 154.86 & 177.80 & 197.80 & 11.85 & 1.20 & 10.30 & 11.01 & 11.83 & 12.66 & 13.39 \\
\hline$\sum$ SFA & 592.03 & 107.59 & 459.90 & 514.34 & 584.24 & 656.79 & 730.49 & 44.59 & 1.32 & 42.96 & 43.82 & 44.64 & 45.46 & 46.17 \\
\hline Total fatty acids & 1327.5 & 236.0 & 1033.4 & 1164.9 & 1313.7 & 1470.4 & 1621.7 & 100.00 & & & & & & \\
\hline
\end{tabular}

${ }^{\top}$ Mean \pm SD gestational age was $11.1 \pm 2.8$ wk. For legend see Table 2.2 (Chapter 2). 
Appendix B: The polyunsaturated fatty acid composition of maternal plasma phospholipids during the second trimester of pregnancy $(n=780){ }^{1}$

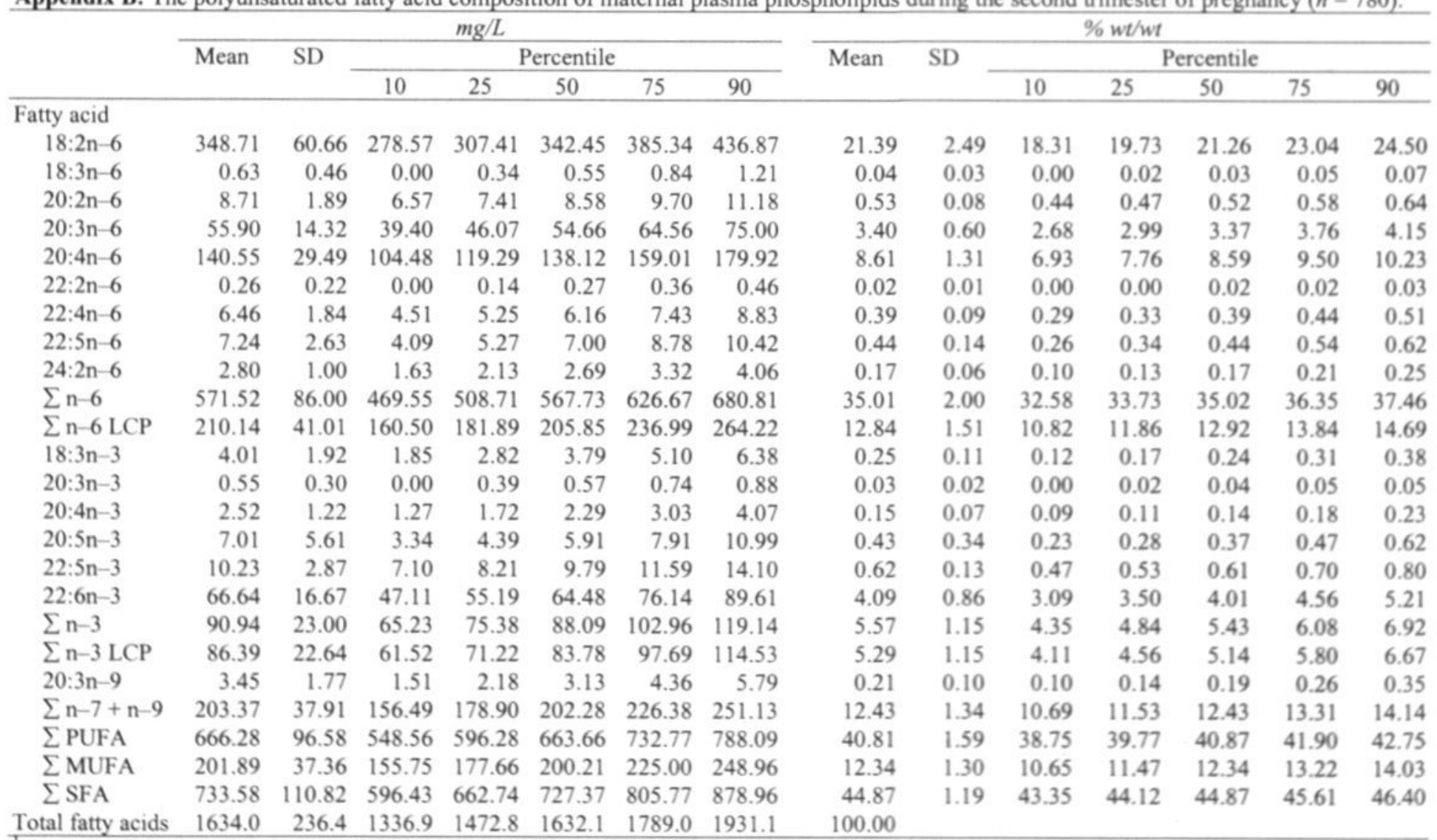

${ }^{\top}$ Mean \pm SD gestational age was $22.5 \pm 1.5$ weeks. For legend see Table 2.2 (Chapter 2). 
Appendix C: The polyunsaturated fatty acid composition of maternal plasma phospholipids during the third trimester of pregnancy $(n=808){ }^{1}$

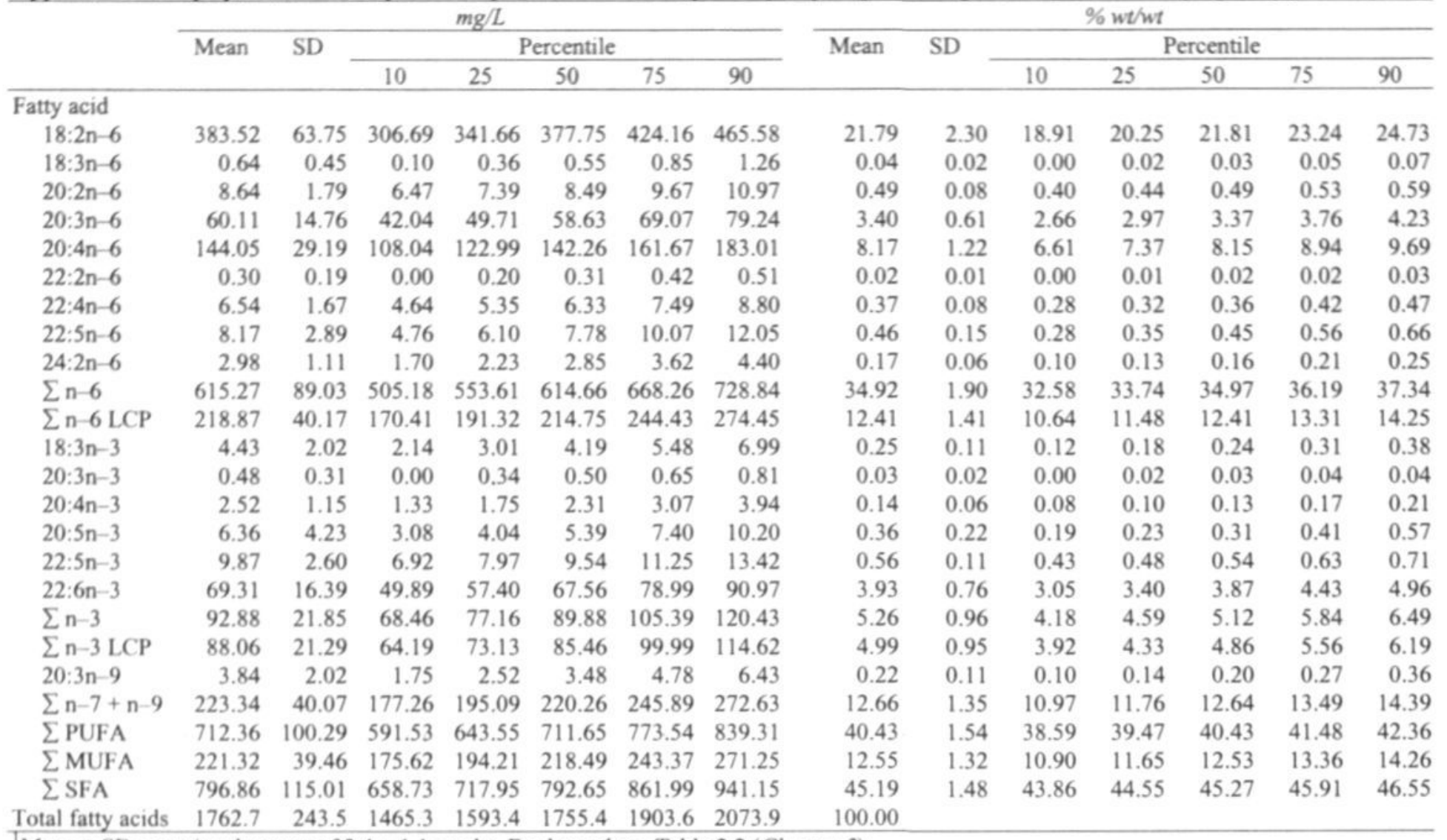

${ }^{T}$ Mean \pm SD gestational age was $32.4 \pm 1.1$ weeks. For legend see Table 2.2 (Chapter 2). 
Appendix D: The polyunsaturated fatty acid composition of plasma phospholipids in 7-year-old boys $(n=145))^{1}$

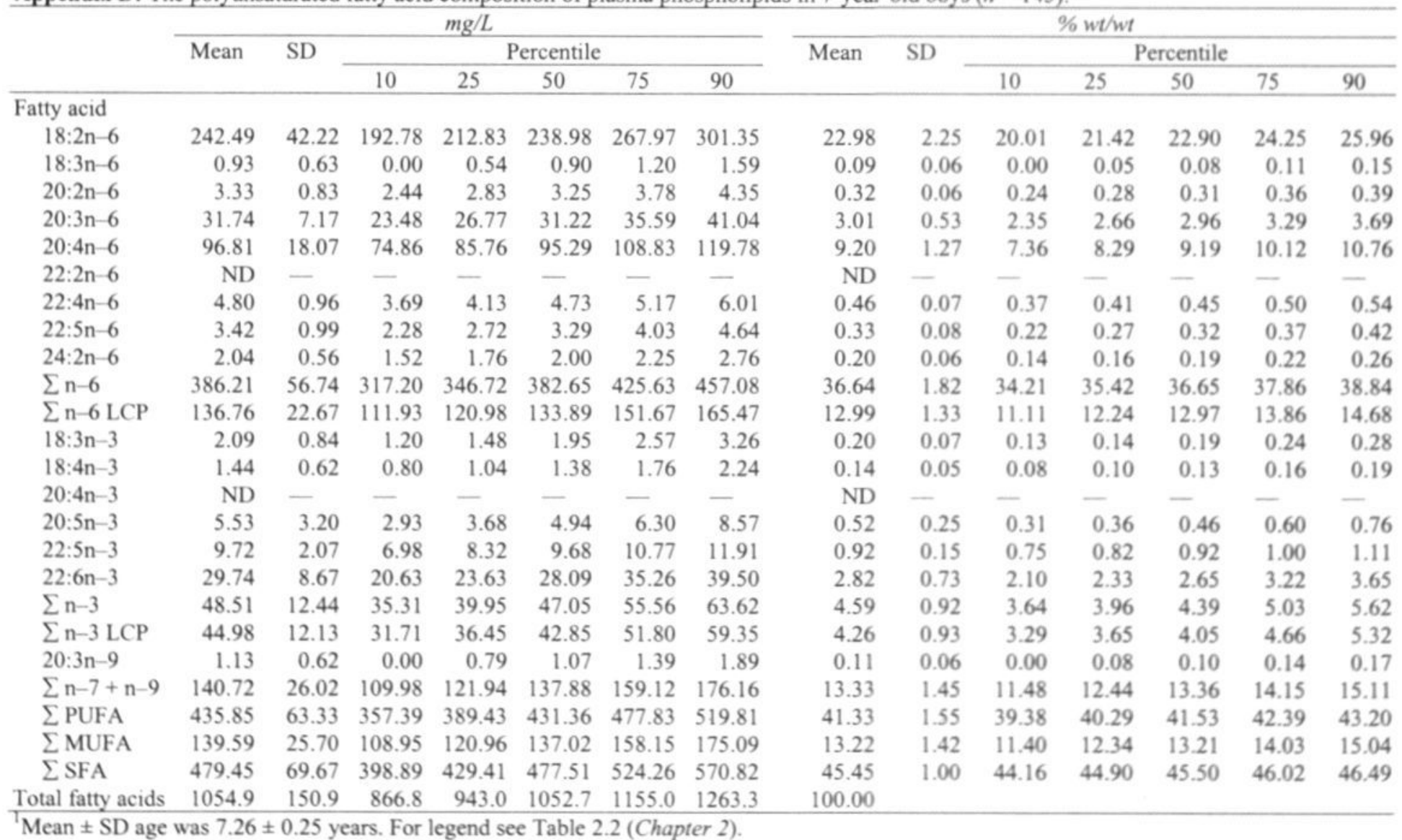

Mean \pm SD age was $7.26 \pm 0.25$ years. For legend see Table 2.2 (Chapter 2). 
Appendix E: The polyunsaturated fatty acid composition of plasma phospholipids in 7-year-old girls $(n=118){ }^{1}$

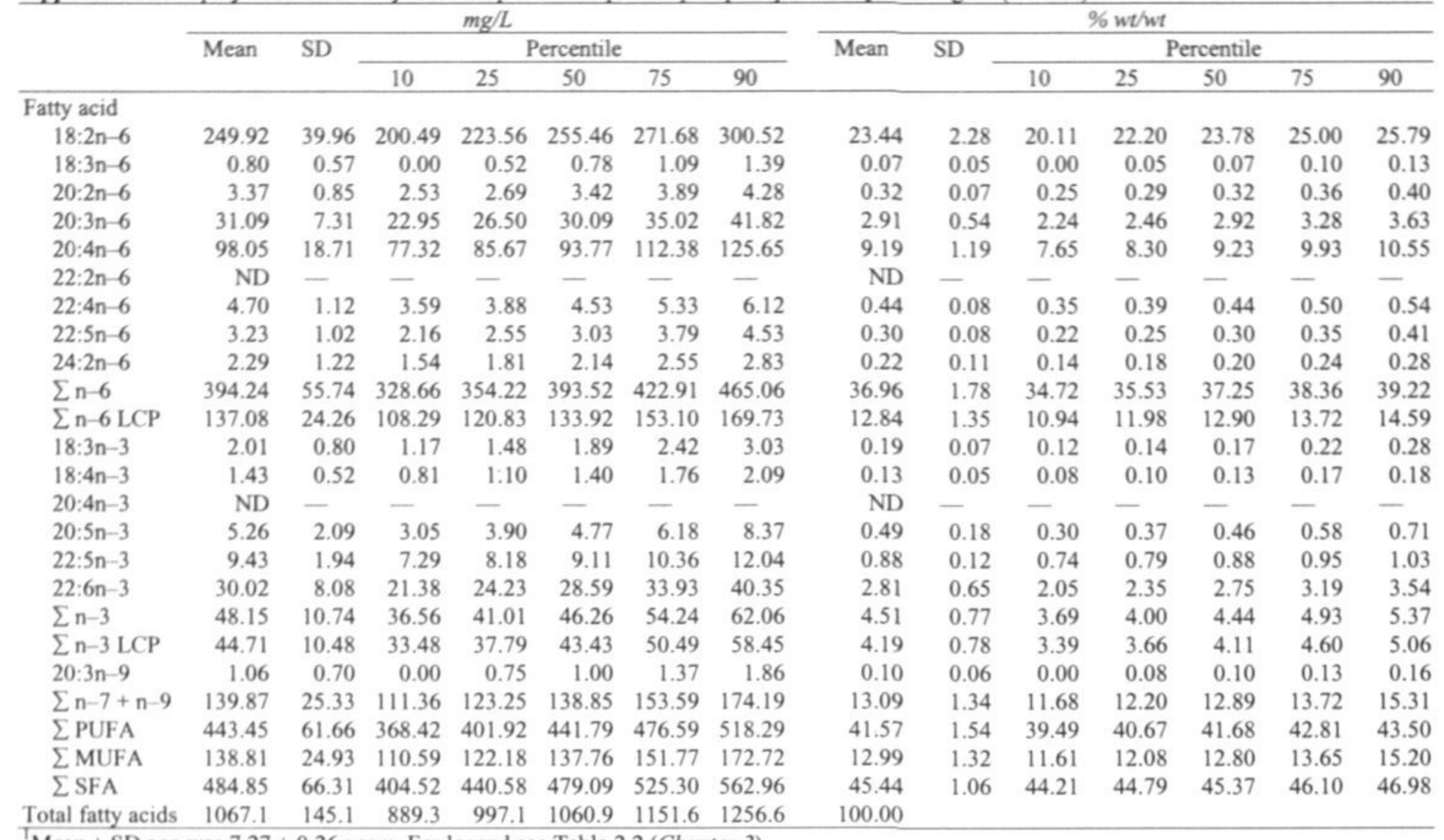

Mean \pm SD age was $7.27 \pm 0.26$ years. For legend see Table 2.2 (Chapter 2 ). 
Appendix F: Distribution of anthropometric characteristics and cardiovascular risk indicators in 7-year-old boys and girls.

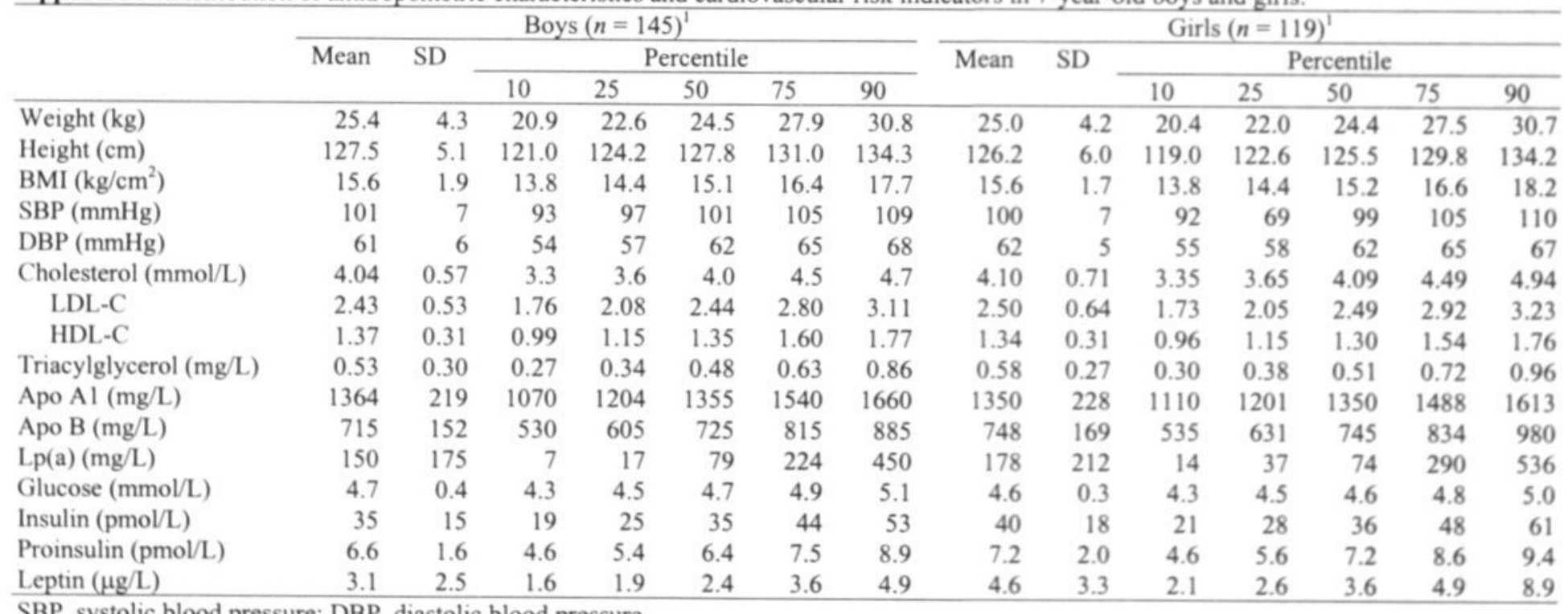

SBP, systolic blood pressure; DBP, diastolic blood pressure.

'For some of the variables the total number of observations may be lower due to missing data. 


\section{Appendices}

Appendix G: The figures on the following two pages depict the distribution of the umbilical cord plasma phospholipid concentration (in $\% \mathrm{wt} / \mathrm{wt}$ and in $\mathrm{mg} / \mathrm{L}$ ) of linoleic acid $(\mathrm{A} ; 18: 2 \mathrm{n}-6)$, dihomo- $\gamma$ linolenic acid (B: 20:3n-6), arachidonic acid (C, 20:4n-6), docosatetraenoic acid (D; 22:4n-6), eicosapentaenoic acid $(E ; 20: 5 n-3)$, docosapentaenoic acid $(F ; 22: 5 n-3)$, docosa-hexaenoic acid (G, $22: 6 n-3)$ and eicosatrienoic acid $(H ; 20: 3 n-9)$ as a function of gestational age at birth. The figures on page 173 depict the distribution of the umbilical cord plasma phospholipid $\sum n-3+\sum n-6 / \sum n-7+$ $\sum n-9,22: 6 n 3 / 22: 5 n 6$, and $\sum n-3 / \sum n-6$ ratio's in relation to gestational age at birth. The number of infants in each gestational age group is given underneath the figure. The lines represent the $10^{\text {th }}, 25^{\text {th }}$, $50^{\text {th }}, 75^{\text {th }}$ and $90^{\text {th }}$ percentiles $(\mathrm{P})$. The triangles represent the $25^{\text {th }}, 50^{\text {th }}$ and $75^{\text {th }}$ percentiles for the preterm infants (average gestational age at birth: $35 \pm 1.5 \mathrm{wk}, n=39$ ). For more information see Chapter 2. 

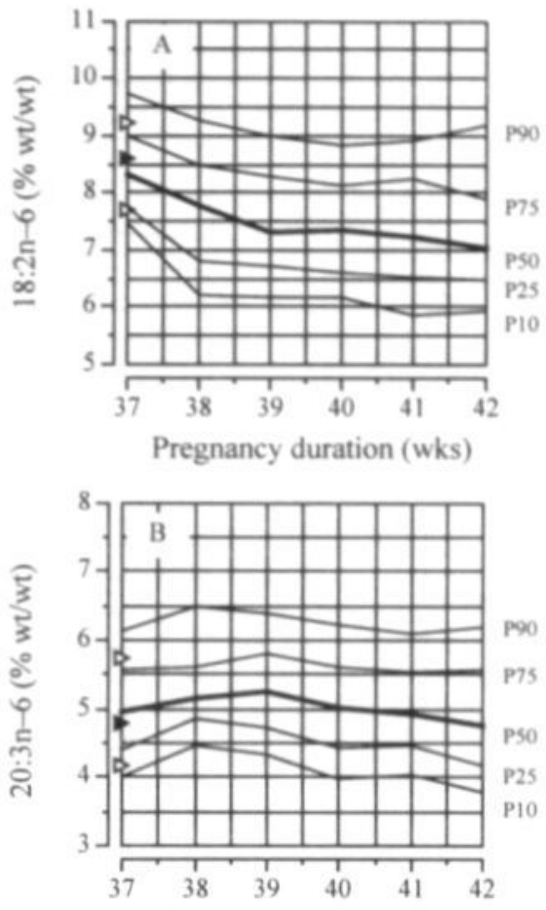

Pregnancy duration (wks)

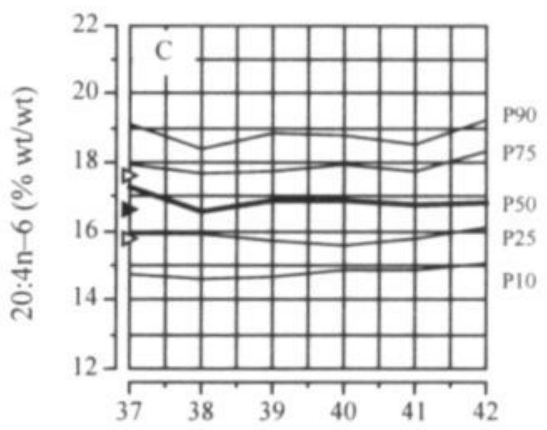

Pregnancy duration (wks)

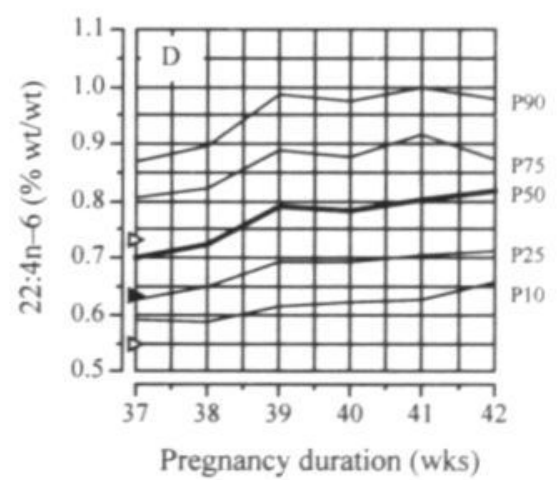

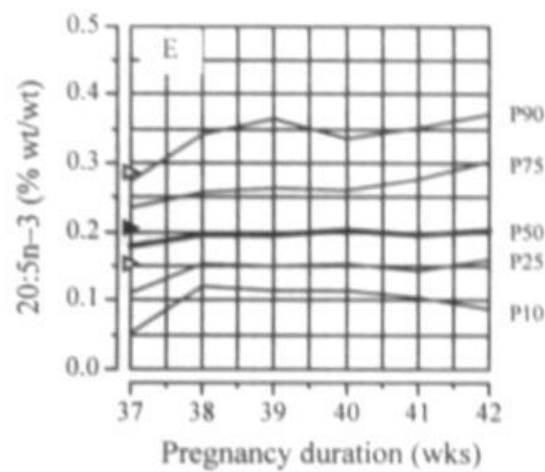
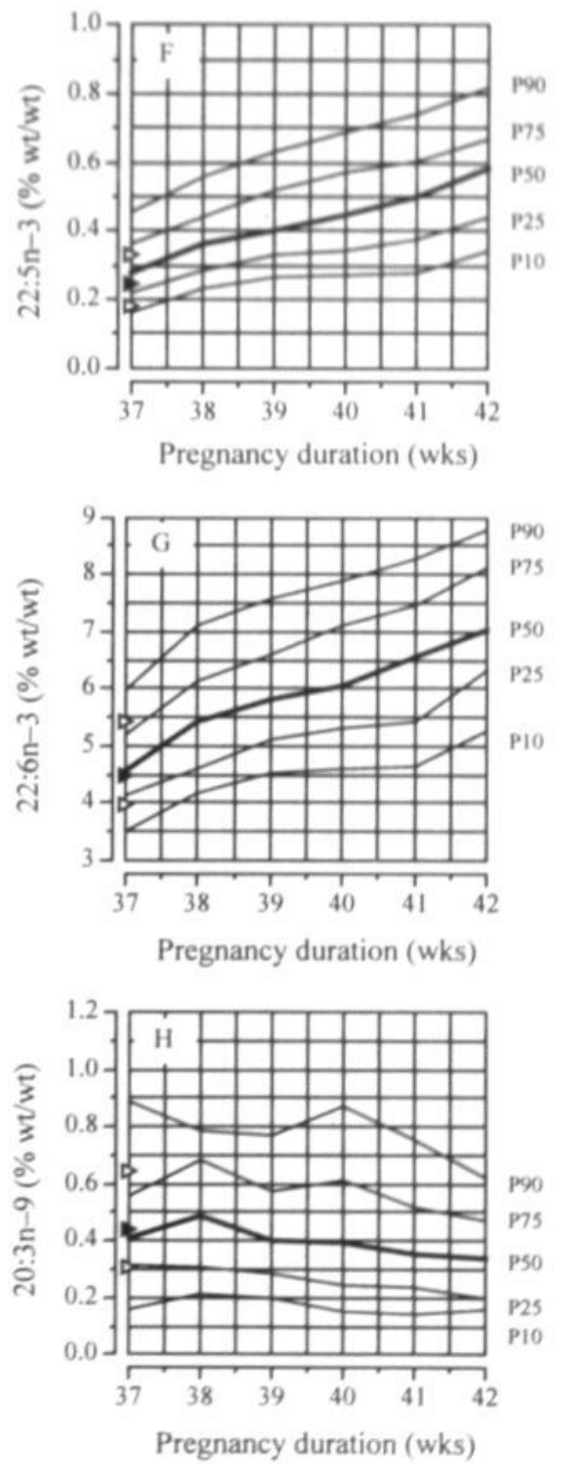

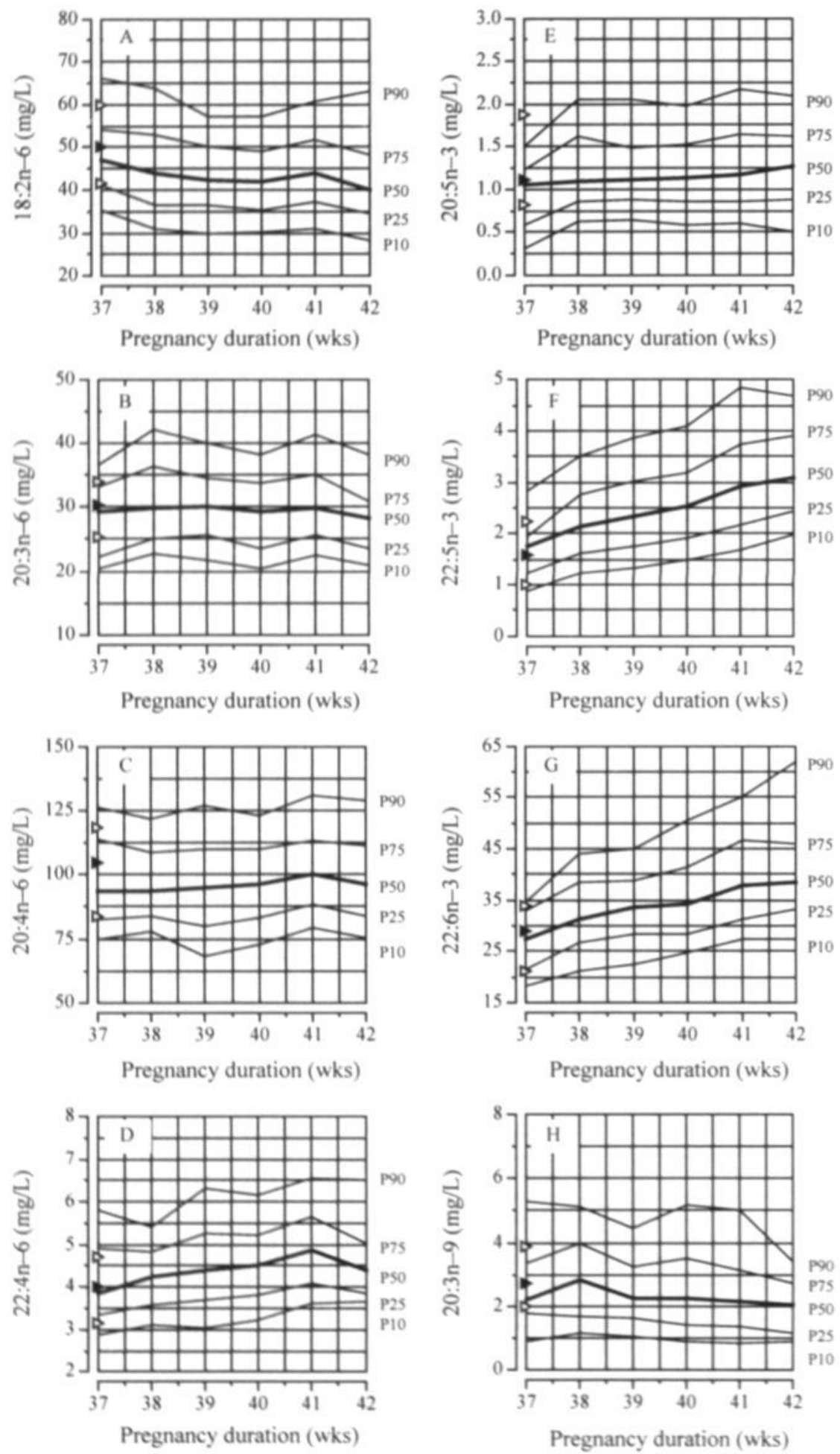

$\begin{array}{lllllll}n: & 32 & 86 & 200 & 233 & 184 & 45\end{array}$ 

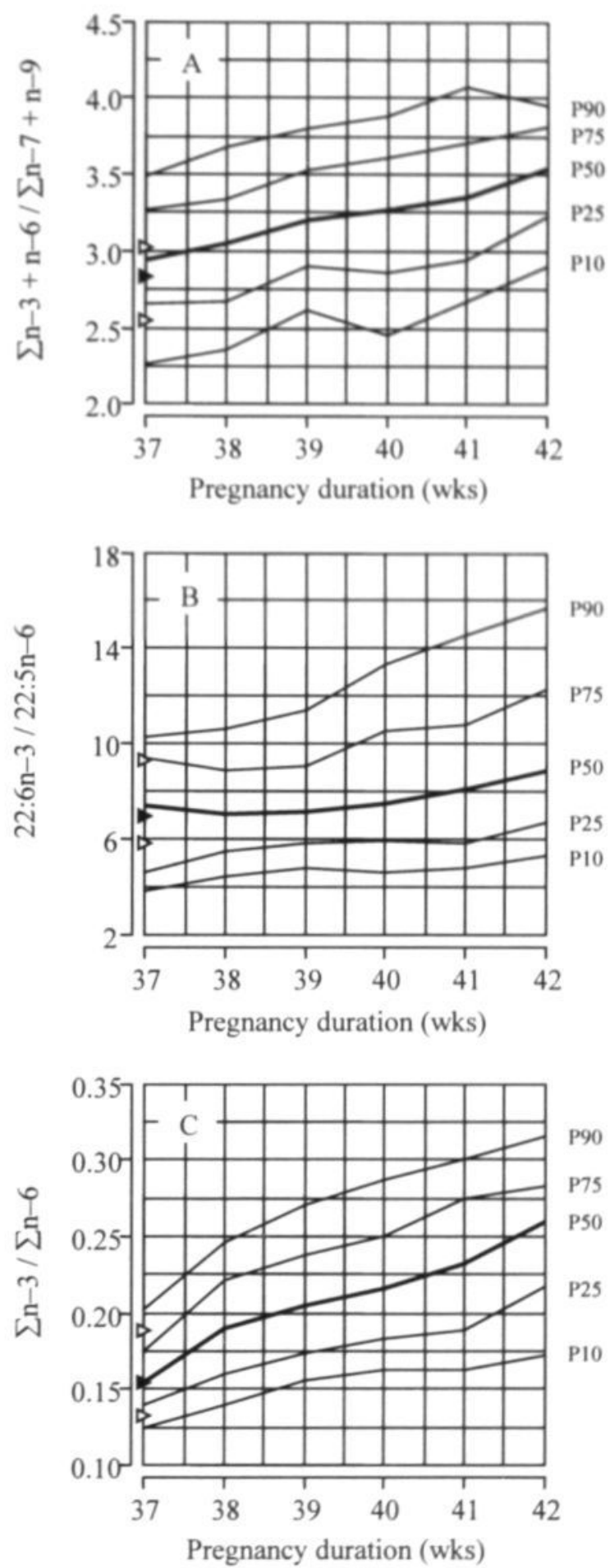

$\begin{array}{lllllll}n: & 32 & 86 & 200 & 233 & 184 & 45\end{array}$ 
Appendices 
Notes | 

